\title{
Investigation of the Amorphization of iron and austenitic stainless steel films by supersaturation with Boron, Carbon, Nitrogen and Oxygen
}

\author{
Dissertation \\ zur Erlangung des Doktorgrades \\ der Mathematisch-Naturwissenschaftlichen Fakultäten \\ der Georg-August-Universität zu Göttingen
}

vorgelegt von

Salvatore Cusenza

aus Wolfsburg - Deutschland

Göttingen 2008 
D 7

Referent: Prof. Dr. Peter Schaaf

Korreferent: $\quad$ Prof. Dr. Reiner Kirchheim

Tag der mündlichen Prüfung: 14.11 .2008 
Physics is like sex-sure, there is some pracitcal results we get from it, but that's not the reason why we do it.

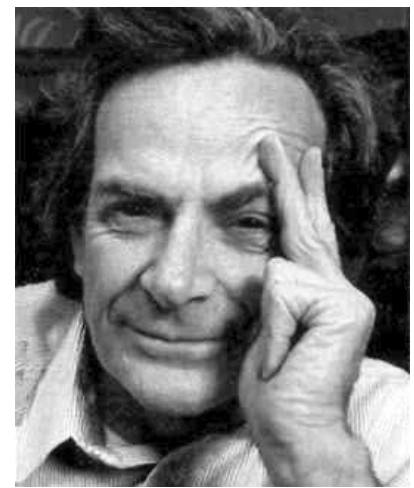

Richard P. Feynman 



\section{Contents}

1 Introduction $\quad 21$

2 Relevant phase diagrams and structures $\quad 23$

2.1 The Fe-C and Fe-N System . . . . . . . . . . . . . . . . . . . . . . . . . . . . . 23

2.2 The Fe-O System . . . . . . . . . . . . . . . . . . . . . . . . . . . . . 24

2.3 The Fe-B System . . . . . . . . . . . . . . . . . . . . . . . . . . . . . . . 24

2.4 The Fe-Cr-Ni System . . . . . . . . . . . . . . . . . . . . . . . . . . . . 25

3 Amorphization of metals $\quad 27$

3.1 Metallic glass formation . . . . . . . . . . . . . . . . . . . . . 27

3.2 Theory of metallic glass formation . . . . . . . . . . . . . . . . . . . . . 28

4 Deposition of thin films and experimental methods $\quad 31$

4.1 Film Deposition . . . . . . . . . . . . . . . . . . . . . . 31

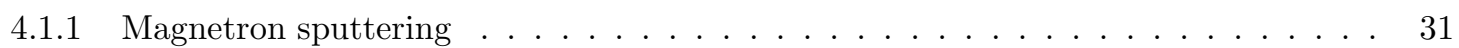

4.1 .2 Pulsed Laser Deposition $(\mathrm{PLD}) \ldots \ldots \ldots$. . . . . . . . . . . . . 32

4.1.2.1 Description of physical phenomena during PLD processing . . . . . . . 33

4.2 Free Electron Laser surface processing . . . . . . . . . . . . . . . . . . . . . . . . . . 33

4.3 Experimental Methods . . . . . . . . . . . . . . . . . . . . . 35

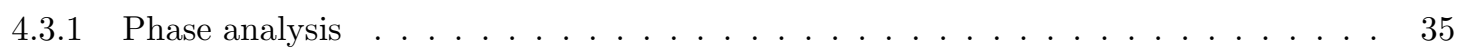

4.3.1.1 Mössbauer Spectroscopy _. . . . . . . . . . . . . . 35

4.3.1.2 Extended X-ray Absorbtion Fine-Structure . . . . . . . . . . . . . 37

4.3 .1 .3 X-ray diffraction . . . . . . . . . . . . . . 38

4.3.1.4 Transmission Electron Microscopy . . . . . . . . . . . . . . . 40

4.3 .2 Chemical composition analysis . . . . . . . . . . . . . . . . . . . 42

4.3.2.1 Rutherford Backscattering Spectrometry . . . . . . . . . . . 42

4.3.2.2 Resonant Nuclear Reaction Analysis . . . . . . . . . . . . . . . . 42

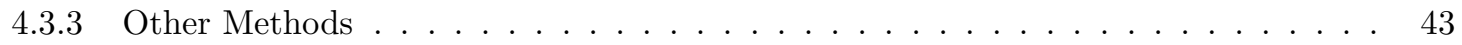

5 Targets and starting materials $\quad 45$ 
$6 \quad$ Results for magnetron-sputtered films $\quad 47$

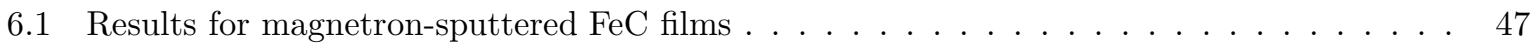

6.1.1 Deposition at room temperature and influence of the Ar gas flow . . . . . . . . 48

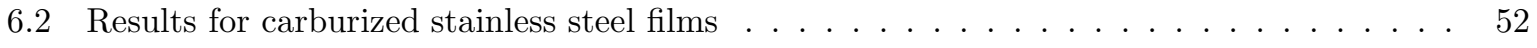

6.2.1 Deposition at room temperature and influence of the methane gas flow . . . . . . 52

6.2.2 Vacuum annealing of films carburized at room temperature . . . . . . . . 56

6.2 .3 The influence of deposition temperature . . . . . . . . . . . . . . 58

6.2.4 Corrosion tests of carburized stainless steel films f . . . . . . . . . . . . 59

6.2 .5 Microhardness of carburized stainless steel films . . . . . . . . . . . . 60

6.2.6 Discussion on a nucleation model for carburized stainless steel films . . . . . . . 60

6.2.7 Microstructure of the amorphous and soft ferromagnetic phase . . . . . . . . 62

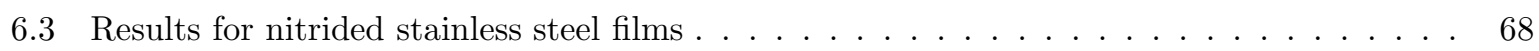

6.3.1 Deposition at room temperature and influence of the nitrogen gas flow . . . . . 68

6.3.2 PAC characterization of the vacuum annealed soft ferromagnetic phase . . . . . . 72

6.3.3 Microhardness of nitrided stainless steel films . . . . . . . . . . . . . . . . 72

6.3.4 Discussion on a nucleation model for nitrided stainless steel films . . . . . . . . . 73

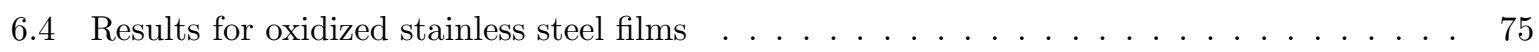

6.4.1 Deposition at room temperature and influence of the oxygen gas flow . . . . . 75

6.4.2 PAC characterization of the vacuum annealed soft ferromagnetic phase . . . . . . 78

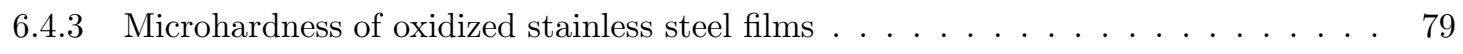

6.4.4 Discussion on a nucleation model for oxidized stainless steel films . . . . . . . . . . 80

6.4.5 Summary of the amorphization process and nucleation model of Magnetronsputtered stainless steel films . . . . . . . . . . . . . . . . . . 81

$\begin{array}{lll}7 & \text { Results for pulsed laser deposited films } & \mathbf{8 3}\end{array}$

Results using the Siemens XP2020 excimer laser . . . . . . . . . . . . . 83

7.1 Results for $\mathrm{FeC}$ films prepared by STPLD . . . . . . . . . . . . . . . . . 83

7.2 Results for carburized stainless steel films prepared by RPLD _ . . . . . . . . . . 87

7.2.1 Deposition at room temperature and influence of the methane gas flow . . . . . . 87

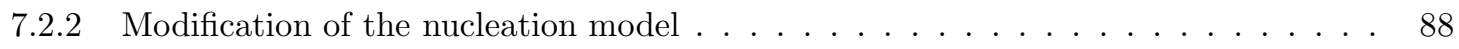

Results using the Quantel Brilliant Nd:YAG laser . . . . . . . . . . . . . 89

7.3 Results for carburized stainless steel films prepared by RPLD . . . . . . . . . . . . . . 89

7.3.1 Deposition at room temperature and influence of the methane gas flow . . . . . . 89

7.3 .2 Microhardness of the RPLD films . . . . . . . . . . . . . . . . . . . . 91

7.4 Self-organized structures in carbon-stainless steel multilayer films . . . . . . . . . . . . 92

7.4.1 Deposition at room temperature and influence of the carbon area fraction . . . . . 92

7.4.2 Influence of the scan frequency on the multilayer thickness . . . . . . . . . . . 96

7.4.3 Thermal stability of carbon-stainless steel multilayer films . . . . . . . . . . . 100 
7.4.4 The quality of the carbon-stainless steel multilayer . . . . . . . . . . . . . . . . 103

7.4.5 Self-organization and nucleation model of carbon-stainless steel multilayer films . . 104

7.5 Results for boron - implanted and boro - nitrided stainless steel films . . . . . . . . . . 107

7.5.1 Results for the implanted steel samples . . . . . . . . . . . . . . . 109

7.5.2 Results for boro-nitrided stainless steel films . . . . . . . . . . . . . . . . 110

7.6 Summary of the pulsed laser deposited films . . . . . . . . . . . . . . . . . . 115

$\begin{array}{lll}8 & \text { FEL surface processing } & 117\end{array}$

9 Summary and Outlook $\quad 121$

$\begin{array}{lr}\text { Bibliography } & 129\end{array}$ 



\section{List of Figures}

1.1 Relation between the critical cooling rate $\left(R_{C}\right)$ for glass formation, the maximum sample thickness $\left(t_{\max }\right)$ and the ratio of liquidus and melting temperature $\left(T_{l} / T_{m}\right)[5] . \ldots 21$

2.1 The Fe-C phase diagram $[15] \ldots \ldots \ldots \ldots \ldots \ldots$

2.2 The Fe-N phase diagram $[15] \ldots \ldots \ldots \ldots \ldots \ldots \ldots \ldots$. . . . . . . . . . . . . . . . 24

2.3 The Fe-O phase diagram $[15] \ldots \ldots \ldots \ldots \ldots \ldots \ldots$

2.4 The Fe-B phase diagram $[15] \ldots \ldots \ldots \ldots \ldots$. . . . . . . . . . . . . . . . 24

2.5 Fe-Cr-Ni phase diagram at $900^{\circ} \mathrm{C}[18] \ldots \ldots \ldots \ldots \ldots \ldots \ldots$

3.1 Viscosity $(\eta)$-temperature $(T)$ dependence corresponding to crystallization and vitrification of a metallic melt. . . . . . . . . . . . . . . . . . . . 27

3.2 Temperature dependence of enthalpy $H$ and specific heat $C_{p}$ corresponding to crystallization and vitrification of a metallic melt. . . . . . . . . . . . . . . . . . . . 28

3.3 Illustration of atomic arrangements in an amorphous solid and the corresponding radial

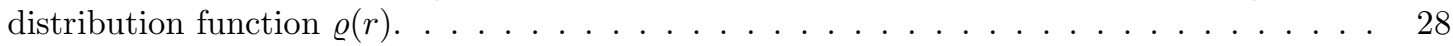

4.1 Principle setup of a planar magnetron system. . . . . . . . . . . . . . . . . . . . . 31

4.2 Three different mechanisms for reactive sputter deposition: (a) at the target, (b) in the plasma volume, (c) at the substrate. . . . . . . . . . . . . . . . . 32

4.3 Principle setup of the PLD system (left) and typical PLD plasma (right). . . . . . . . . 33

4.4 Principle setup of the FEL system used at the Jefferson lab. . . . . . . . . . . . . . . . . . 34

4.5 Quadrupole splitting for a $3 / 2$ to $1 / 2$ transition. The magnitude of quadrupole splitting $\Delta$ is shown. . . . . . . . . . . . . . . . . . . 36

4.6 Magnetic splitting of the nuclear energy levels. . . . . . . . . . . . . . . . . . 36

4.7 Process scheme of the internal conversion for ${ }^{57} \mathrm{Fe} . \quad \ldots \ldots \ldots$. . . . . . . . . . . . 37

4.8 Mechanical drawing of the CEMS detector. . . . . . . . . . . . . . . . . . 37

4.9 Typical XAFS spectrum for Fe. The XANES and EXAFS regions are identified. $\mu(E)$ is shown with the smooth background function $\mu_{0}(E)$ and the edge step $\Delta \mu_{0}\left(E_{0}\right) \ldots \ldots$

4.10 Visualization of the so-called Bragg equation. Maximum scattered intensity is only obtained when the phase shifts add to a multiple of the incident wavelength $\lambda . \ldots$. . . . 38

4.11 Laue diffraction geometry. . . . . . . . . . . . . . . . . . . . . . . . . 39

4.12 Vectorial display of the Laue diffraction geometry. . . . . . . . . . . . . . . . . . . 39

4.13 The $\theta / 2 \theta$ geometry. The x-ray tube is operated in the line focus mode; the plane of the figure is the scattering plane. . . . . . . . . . . . . . . . . . 39 
4.14 The GIXRD geometry. The x-ray tube is operated in the line focus mode; the plane of the figure is the scattering plane. . . . . . . . . . . . . . . . . . 40

4.15 The wavelength $\lambda$ of the electrons as a function of the accelerating voltage. . . . . . . . 41

4.16 The wavelength $\lambda$ of the electrons as a function of the accelerating voltage. . . . . . . . 41

4.17 Schematics of the IONAS accelerator. . . . . . . . . . . . . . . . . 43

4.18 Schematics of the RNRA process. . . . . . . . . . . . . . . . . . . . 43

5.1 top: GIXRD $\left(2^{\circ}\right.$ incidence angle) pattern of the ARMCO-Iron sputter target; bottom: $\theta-2 \theta$ pattern of the AISI 316 sputter target. . . . . . . . . . . . . . 45

5.2 (a) Transmission Mössbauer spectrum of the original ARMCO-Iron sputter target; (b) CEM spectrum of the original AISI 316 sputter target. The corresponding distributions of the hyperfine fiels $p(B)$ are given on the right. . . . . . . . . . . . . . . .

5.3 Angular scans of ARMCO-Iron sputter target: (top) coercitive field $H_{C}$; (middle) relative remanence $M_{r} / M_{s} ;$ (bottom) magnetization energy density $E_{s} / M_{s} \ldots \ldots$. . . . . .

6.1 Sputtering-yield $Y$ of Fe, $\mathrm{C}$ and the sum of both elements in dependence of the $\mathrm{Ar}^{+}$ion energy. . . . . . . . . . . . . . . . . . . . . 47

6.2 The $\mathrm{Fe}_{50} \mathrm{C}_{50}$ Magnetron sputter target. . . . . . . . . . . . . . . . . . . . . . . . 47

6.3 Growth rate $g$ as a function of the Ar gas flow $j_{\mathrm{Ar}}$ for the room temperature deposition. . 48

6.4 GIXRD $\left(2^{\circ}\right)$ spectra of the inert sputterd samples. The Ar gas flows are given in the graphs. 48

6.5 Peak position and peak width of the first broad peak in the XRD spectra of the reactive sputtered stainless steel films. . . . . . . . . . . . . . . . . . . . . . 48

6.6 Mössbauer spectra of the inert sputtered FeC films. . . . . . . . . . . . . . . . . . . . . . 49

6.7 Hysteresis curve of the inert deposited FeC film sputtered at $50 \mathrm{sccm}$ Ar gas flow. . . . . 50

6.8 STM pattern for the FeC sample sputtered at $140 \mathrm{sccm}$ Ar gas flow. . . . . . . . . . . 50

6.9 Autocorrelation of the STM image as shown in Fig. 6.8 . . . . . . . . . . . . . . . 50

6.10 Power-spectral density diagram derived from the line scan, which is shown in Fig. 6.9. The parameters of the Gauss multi-peak fit are given in the graph. . . . . . . . . . . . . . 50

6.11 FeC film after deposition. The numbers indicate the positions of the RBS measurements. 50

6.12 Mean concentrations as derived from the RBS fitting routine. . . . . . . . . . . . 51

6.13 Poisoning of the target as a function of the sputter yields of $\mathrm{Fe}$ and $\mathrm{C}$ at fixed $E_{A r^{+}}=$ $400 \mathrm{eV}$. The black dashed line corresponds to the $\mathrm{Fe}_{50} \mathrm{C}_{50}$ stoichiometry. . . . . . . . . 51

6.14 RBS spectra of the target before (black) and after (red dashed) sputtering. . . . . . . . 51

6.15 Growth rate $g$ as a function of the $\mathrm{CH}_{4}$ gas flow $j_{\mathrm{CH}_{4}}$ for the room temperature deposition. 52

6.16 GIXRD $\left(2^{\circ}\right)$ spectra of the carburized films. The $\mathrm{CH}_{4}$ gas flows are given in the graph. $\quad 52$

6.17 Peak position and peak width of the first broad peak in the XRD spectra of the reactive sputtered stainless steel films. . . . . . . . . . . . . . . . . . 53

6.18 Angular scans of (left) $0.01 \mathrm{sccm} \mathrm{CH}_{4}$ and (right) $0.10 \mathrm{sccm} \mathrm{CH}_{4}$ gas flow. The coercive field $H_{\mathrm{C}}$ (top) and the relative remanence $M_{\mathrm{R}} / M_{\mathrm{S}}$ (bottom) are shown. . . . . . . . . . .

6.19 Mössbauer spectra of the reactive sputtered AISI 316 films. The numbers in the graphs represent the methane gas flow. . . . . . . . . . . . . . . . . . . 54

6.20 RBS spectra of the reactively sputtered films. The $\mathrm{CH}_{4}$ flow is given in the graph. . . . . 55 
6.21 Carbon content in the reactively sputtered films as derived from the RBS analysis versus the $\mathrm{CH}_{4}$ gas flow. . . . . . . . . . . . . . . . . . . . .

6.22 AFM measurement (left) and cross section analysis (right) of the sample sputtered with a $\mathrm{CH}_{4}$ gas flow of $0.01 \mathrm{sccm}$. The cross section analysis (right bottom) shows the line from which the roughness and the cluster-size were derived. . . . . . . . . . . . . .

6.23 Raman results for the amorphous soft ferromagnetic carburized AISI 310 and AISI 316 films. 56

6.24 Mössbauer spectra of vacuum annealed AISI 316 film sputtered with a magnetron power of $100 \mathrm{~W}$ and $0.01 \mathrm{sccm}$ methane. Exposition time and temperature are given. . . . . . .

6.25 GIXRD $\left(2^{\circ}\right.$ incidence angle) spectra of the post-vacuum annealed samples sputtered with a magnetron power of $100 \mathrm{~W}$ and $0.01 \mathrm{sccm}$ methane. Annealing temperature and time are given.

6.26 SEM pictures of the post-vacuum annealed samples: a) $1 \mathrm{~h}$ at $973 \mathrm{~K}$, b) $10 \mathrm{~h}$ at $973 \mathrm{~K}$ and c) $120 \mathrm{~h}$ at $973 \mathrm{~K} \ldots \ldots \ldots \ldots \ldots \ldots$

6.27 Raman measurements of the post-vacuum annealed samples.

6.29 GIXRD ( $2^{\circ}$ incidence angle) spectra of the as-carburized samples sputtered with a magnetron power of $100 \mathrm{~W}$ and $0.01 \mathrm{sccm}$ methane. The substrate temperatures are given. . .

6.30 Angular scans of the film sputtered at $673 \mathrm{~K}$ with $0.1 \mathrm{sccm} \mathrm{CH}_{4}$ gas flow. The polar diagrams of the coercive field $H_{\mathrm{C}}$ ( left) and the relative remanence $M_{\mathrm{R}} / M_{\mathrm{S}}$ (right) are shown. . . . . . . . . . . . . . . . . . . . . . .

6.28 CEM spectra of carburized AISI 316 films sputtered with a magnetron power of $100 \mathrm{~W}$ and $0.01 \mathrm{sccm}$ methane at two temperatures: $\mathrm{T}=298 \mathrm{~K}$ (top), $\mathrm{T}=673 \mathrm{~K}$ (bottom). On the right hand side, the hyperfine field distribution $p(B)$ and the quadrupole splitting distribution $p(\Delta)$ are shown. . . . . . . . . . . . . . . . .

6.31 Corrosion tests of AISI 310 (a) and AISI 316 (b) sputtered films. All samples were sputtered at a magnetron power of $100 \mathrm{~W}$, inert-sputtered sample with $12 \mathrm{sccm}$ Ar gas flow, carburized samples with $0.01 \mathrm{sccm} \mathrm{CH}_{4}$ gas flow. Temperatures are given in the graphs.

6.32 Peak width of the first amorphous peak in dependence of the carbon content of reactive sputtered stainless steel films. . . . . . . . . . . . . . . . . .

6.34 FIM pattern of the amorphous and soft ferromagnetic film (sputtered at $298 \mathrm{~K}$ at $0.01 \mathrm{sccm}$

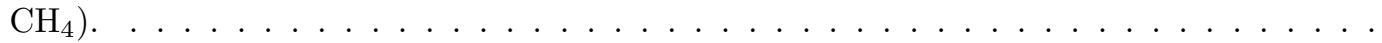

6.33 TEM pattern of the amorphous and soft ferromagnetic film (sputtered at $298 \mathrm{~K}$ at $0.01 \mathrm{sccm}$ $\mathrm{CH}_{4}$ ). a) and b) dark field pattern, c) SAD pattern and d) HR-TEM of the film. . . . . .

6.35 DSC curve of the amorphous and soft ferromagnetic film (sputtered at $298 \mathrm{~K}$ at $0.01 \mathrm{sccm}$ $\mathrm{CH}_{4}$ ) measured at a heating rate of $10 \mathrm{~K} / \mathrm{s}$. the arrow refers to the glass transition temperature $\mathrm{T}_{g} \ldots \ldots \ldots \ldots \ldots \ldots \ldots \ldots \ldots \ldots$

6.36 Fine-structure oscillations weighted with $k^{2}$ factor of: a) $\gamma$-(Fe,Cr,Ni) (bulk AISI 316), b) $\alpha$-Fe, c) $\mathrm{Fe}_{3} \mathrm{C}$ and the reactive magnetron-sputtered sample deposited with $0.01 \mathrm{sccm} \mathrm{CH}_{4}$.

6.37 BFT moduli of bulk AISI 316 (solid) and the amorphous and soft ferromagnetic film (sputtered at $298 \mathrm{~K}$ at $0.01 \mathrm{sccm} \mathrm{CH}_{4}$ ) (dashed line) measured at the Fe K-edge (top) and at the Ni K-edge (bottom). . . . . . . . . . . . . . . . . . . .

$6.38 \mathrm{XRD}$ pattern of the reactive magnetron-sputtered film deposited at $0.01 \mathrm{sccm} \mathrm{CH}_{4}$ gas flow. 65

6.39 Median hyperfine field $\mathrm{B}_{h f}$ in dependence of the carbon content of reactive sputtered stainless steel films. . . . . . . . . . . . . . . . . . .

6.40 Crystal structure of trigonal $\mathrm{Ni}_{3} \mathrm{C}$ phase: space group $\mathrm{R}-3 \mathrm{c}(167), a=4.5530 \AA, c=$

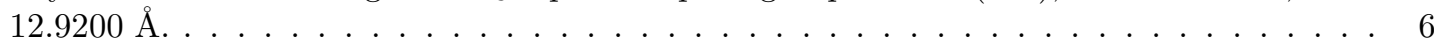

6.41 Growth rate $g$ as a function of the $\mathrm{N}_{2}$ gas flow $j_{\mathrm{N}_{2}}$ for the room temperature deposition (line is only to guide the eye). . . . . . . . . . . . . . . . . 
6.42 GIXRD $\left(2^{\circ}\right)$ spectra of the nitrided films. The $\mathrm{N}_{2}$ gas flows are given in the graph; the peaks of the $\mathrm{ZnS}$-type $(\mathrm{Fe}, \mathrm{Cr}, \mathrm{Ni}) \mathrm{N}$ phase are indexed. . . . . . . . . . . . . .

6.43 Peak position and peak width of the first broad peak in the XRD spectra of the reactive sputtered stainless steel films. . . . . . . . . . . . . . . . . . .

6.44 Mössbauer spectra of the reactive sputtered AISI 316 films. The numbers in the graphs represent the nitrogen gas flow. . . . . . . . . . . . . . . . .

6.45 Angular scans of the reactive sputtered films with: (top) $0.01 \mathrm{sccm} \mathrm{N}_{2}$ and b) (bottom) $0.05 \mathrm{sccm} \mathrm{N}_{2}$ gas flow. The polar diagrams of the coercive field $H_{\mathrm{C}}$ are shown left and the relative remanence $M_{\mathrm{R}} / M_{\mathrm{S}}$ at the right. . . . . . . . . . . . . . . .

6.46 RBS spectra of the reactively sputtered films. The $\mathrm{N}_{2}$ flow is given in the graph. . . . . .

6.47 Nitrogen content in the reactively sputtered films as derived from the RBS analysis versus the $\mathrm{N}_{2}$ gas flow. . . . . . . . . . . . . . . . . . . . . .

6.48 RNRA depth-profiling results of the as-nitrided samples. The $\mathrm{N}_{2}$ gas flow are given in the graph. . . . . . . . . . . . . . . . . . . . . .

$6.49{ }^{111}$ In PAC perturbation spectra along with their Fourier transforms for measurements of the as-nitrided samples: a) (top) PAC measurement of the as-nitrided sample sputtered at $0.05 \mathrm{sccm} \mathrm{N}_{2}$ gas flow and b) (bottom) PAC measurement after 2 hours of post vacuum annealing treatment performed at $T_{a}=973 \mathrm{~K}$. All presented spectra are recorded at $298 \mathrm{~K} .73$

6.50 Dependency between Martens hardness and nitrogen content including data from [132], [133]. The $\mathrm{N}$ values are obtained from the RNRA analysis. . . . . . . . . . . . . . .

6.51 Peak width of the first amorphous peak and reactive gas flow in dependence of the nitrogen content of reactive sputtered stainless steel films. . . . . . . . . . . . . . . .

6.52 Growth rate $g$ as a function of the $\mathrm{O}_{2}$ gas flow $j_{\mathrm{O}_{2}}$ for the room temperature deposition (line is only to guide the eye) . . . . . . . . . . . . . . . . . .

6.53 GIXRD $\left(2^{\circ}\right)$ spectra of the oxidized films. The $\mathrm{O}_{2}$ gas flows are given in the graph. . . .

6.54 Mössbauer spectra of the reactive sputtered AISI 316 films. The numbers in the graphs represent the oxygen gas flow. . . . . . . . . . . . . . . .

6.55 Peak position and peak width of the first broad peak in the XRD spectra of the reactive sputtered stainless steel films. . . . . . . . . . . . . . . . . . .

6.56 Angular scans of the deposited film sputtered at $1.00 \mathrm{sccm} \mathrm{O}_{2}$ gas flow. The polar diagrams of the coercive field $H_{\mathrm{C}}$ (top) and the relative remanence $M_{\mathrm{R}} / M_{\mathrm{S}}$ (bottom) are shown. . .

6.57 RBS spectra of the reactively sputtered films. The $\mathrm{O}_{2}$ flow is given in the graph. . . . . . 77

6.58 Oxygen content in the reactively sputtered films as derived from the RBS analysis versus the $\mathrm{O}_{2}$ gas flow. . . . . . . . . . . . . . . . . . . . . . .

$6.59{ }^{111}$ In PAC perturbation spectra along with their Fourier transforms for measurements: a) (top) PAC measurement of the as-oxidized sample sputtered at $1.00 \mathrm{sccm} \mathrm{O}_{2}$ gas flow and b) (bottom) PAC measurement after 2 hours of post vacuum annealing treatment performed at $T_{a}=973 \mathrm{~K}$. All presented spectra are recorded at $298 \mathrm{~K}$. . . . . . . . .

6.60 Dependency between Martens hardness and oxygen content. The Oxygen values are obtained from the RBS analysis. . . . . . . . . . . . . . . . . . .

6.61 Peak width of the amorphous peak and reactive gas flow in dependence of the oxygen content of reactive sputtered stainless steel films. . . . . . . . . . . . . . . .

6.62 Normalized gas flow in dependence of the radius of the reactive gas atoms. The parameters for the exponential decay fitting routine are given in the graph. . . . . . . . . . . . 
7.1 Different stainless steel STPLD targets in comparison to a conventional stainless steel PLD target (right). The red lines indicate the laser scan area. As indicated in SS/BN 50:50 STPLD target, for ultra-thin films a scan area of $4 \mathrm{~mm}$ (4 mm stainless steel/4 mm C) and $8 \mathrm{~mm}(8 \mathrm{~mm}$ stainless steel $/ 8 \mathrm{~mm} \mathrm{C}$ ) for thin-films were used. . . . . . . . . .

7.4 TEM pattern of the NaCl-type FeC grain and its FFT on the right hand. . . . . . . . . . 84

7.2 (top) HR-TEM pattern of a grain. (bottom) diffraction pattern of this grain. The orientation of the reflexes are given in the graph. . . . . . . . . . . . . . . . . . 84

7.3 Fine $\mathrm{Fe}$ and $\mathrm{C}$ multilayer system surrounding a metallic glassy grain observed by TEM and its FFT of that area (right). . . . . . . . . . . . . . . .

7.5 GIXRD $\left(2^{\circ}\right)$ spectra of the STPLD films deposited at 298 (top) and $823 \mathrm{~K}$ (bottom). The temperatures and reflexes are given in the graph. . . . . . . . . . . . . 85

7.7 Median hyperfine field $\mathrm{B}_{\mathrm{hf}}$ in dependence of the carbon content. . . . . . . . . . . . 85

7.6 Mössbauer spectra of the STPLD deposited FeC films at $298 \mathrm{~K}$ (top) and $823 \mathrm{~K}$ (bottom). 86

7.8 RBS spectra of the STPLD films deposited at 298 and 823 K . . . . . . . . . . . 86

7.9 GIXRD $\left(2^{\circ}\right)$ spectra of the RPLD film. The reflexes are given in the graph. . . . . . . . . 87

7.10 CEM spectrum of the RPLD sample. The $\mathrm{CH}_{4} /$ chamber pressure is given in the graph. . 88

7.11 RBS spectra of the RPLD films. The $\mathrm{CH}_{4}$ /chamber pressure is given in the graph. . . . 88

7.12 Growth rate $g$ as a function of the $\mathrm{CH}_{4} /$ chamber pressure for the room temperature de-

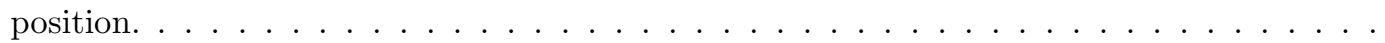

7.15 Mössbauer spectra of the reactive sputtered AISI 316 films. The numbers in the graphs represent the methane gas flow. . . . . . . . . . . . . . . . .

7.13 GIXRD $\left(2^{\circ}\right)$ spectra of the carburized RPLD films. The $\mathrm{CH}_{4} /$ chamber pressure are given

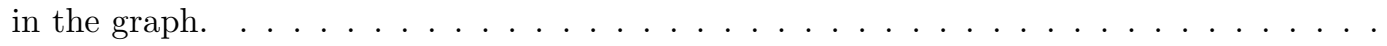

7.14 Peak position and peak width of the first broad peak in the XRD spectra of the RPLD stainless steel films. . . . . . . . . . . . . . . . . . . . . . 90

7.16 RBS spectra of the RPLD films. The $\mathrm{CH}_{4}$ / chamber pressure is given in the graph. . . . . 91

7.17 Carbon content in the RPLD films (as derived from the RBS analysis) versus the

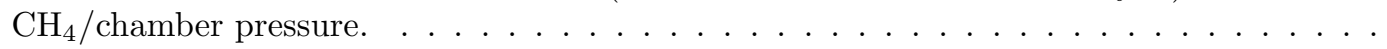

7.18 Dependency between Martens hardness and carbon content (as derived from the RBS

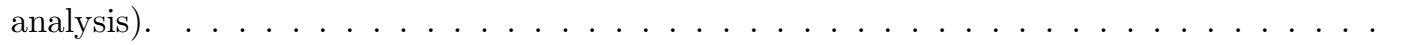

7.19 Growth rate $g$ as a function of the carbon area fraction for the room temperature STPLD

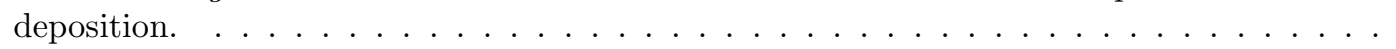

7.22 Mössbauer spectra of the STPLD stainless steel films. The numbers in the graphs represent the stainless steel/carbon area fraction of the STPLD targets. . . . . . . . . . . .

7.23 Polar diagrams of the coercive field $H_{\mathrm{C}}$ and the relative remanence $M_{\mathrm{R}} / M_{\mathrm{S}}$ for the STPLD 90:10 (left), the 60:40 (middle) and the 50:50 sample (right).

7.26 TEM pattern of the STPLD 9010 sample. Left: overview of the sample; middle: HR-TEM image of film/interface region; right: HR-TEM image of the surface region. . . . . . . . .

7.20 GIXRD $\left(2^{\circ}\right)$ spectra of the STPLD films. The stainless steel/carbon area fractions are given in the graph. . . . . . . . . . . . . . . . . . . . 9

7.21 Peak position and peak width of the first broad peak in the XRD spectra of the STPLD stainless steel films. . . . . . . . . . . . . . . . .

7.24 RBS spectra of the STPLD films. The stainless steel/carbon area ratios are given in the

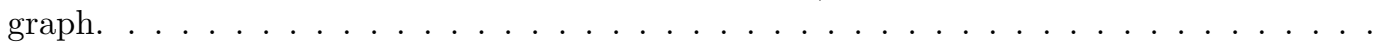


7.25 RBS depth profile of the STPLD 90:10 sample. . . . . . . . . . . . . . . . . .

7.27 Comparison between conventional and modified 60:40 STPLD films: Mössbauer spectra (top); TEM pattern (middle) and RBS spectra with calculated depth profile (bottom). . .

7.28 Comparison between conventional and modified 50:50 STPLD films: Mössbauer spectra (top); TEM pattern (middle) and RBS spectra with calculated depth profile (bottom). . .

7.29 GIXRD ( $2^{\circ}$ incidence angle) spectrum of the post-vacuum annealed STPLD film. Phase, annealing temperature and time are given in the graph. . . . . . . . . . . . . 100

7.30 CEM spectrum of the post-vacuum annealed 90:10 STPLD film. . . . . . . . . . . . 100

7.31 TEM patterns of the post-vacuum annealed 90:10 STPLD film. . . . . . . . . . . . 100

7.32 RBS spectra of the post-vacuum annealed and as-deposited 90:10 STPLD film. the depth profile of the post-vacuum annealed can be found at the bottom . . . . . . . . . . . 101

7.33 GIXRD ( $2^{\circ}$ incidence angle) spectrum of the 90:10 STPLD film deposited with an substrate temperature of $673 \mathrm{~K} \ldots \ldots \ldots \ldots 10 . \ldots \ldots$

7.34 CEM spectrum of the 90:10 STPLD film deposited with an substrate temperature of 673 K.102

7.35 RBS spectra of the 90:10 STPLD film deposited at 298 and $673 \mathrm{~K}$. The depth profile of the film deposited at $673 \mathrm{~K}$ can be found at the bottom. . . . . . . . . . . . . . . 102

7.36 TEM patterns of the 90:10 STPLD sample deposited at $673 \mathrm{~K}$. Left: overview of the sample; right: HR-TEM pattern. . . . . . . . . . . . . . . . . . . 103

7.37 X-ray reflectivity pattern of the 50:50mod STPLD film. . . . . . . . . . . . 103

7.38 RBS spectra of the 50:50, 50:50mod and 50:50modwob STPLD film deposited at $298 \mathrm{~K}$. The depth profile of the 50:50modwob STPLD film can be found at the bottom. . . . . . 104

7.39 EDX-time to space correlation diagram of the 90:10 STPLD film. The dashed line corresponds to the situation, in which the laser spot hits the graphite layer of the target. . . . 10

7.40 EDX-time to space correlation diagram of the 50:50 STPLD film. The dashed line corresponds to the situation, in which the laser spot hits the graphite layer of the target. . . . 105

7.41 Ion distribution of the plasma ions of the $90: 10$ target. . . . . . . . . . . . . . 105

7.42 Ion distribution of the plasma ions of the $50: 50$ target. . . . . . . . . . . . . . 106

7.43 RBS spectra of the Aluminum bulk material and the Al/C 60:40mod STPLD film deposited at $298 \mathrm{~K}$. The depth profile of the Al/C 60:40mod STPLD film can be found at the bottom. 106

7.44 RBS spectra of the Titanium bulk material, the Ti/C 90:10 and the Ti/C 60:40mod STPLD films deposited at $298 \mathrm{~K}$. The depth profile of the Ti/C 90:10 and of the Ti/C 60:40mod STPLD film can be found at the middle/bottom. . . . . . . . . . . . . . 107

7.45 Dependence between multilayer thickness and atomic number at constant carbon content. Here, the thicknesses of SS/C, Al/C and Ti/C 60:40mod samples are summarized. . . . . 107

7.46 Implantation profiles calculated by SRIM for stainless steel samples containng 1, 3 and 6 at.\% boron. . . . . . . . . . . . . . . . . . . . 108

7.47 Growth rate $g$ as a function of the boron-nitride area fraction for the room temperature STPLD deposition. . . . . . . . . . . . . . . . . . . . 108

7.48 RBS spectra of AISI 316 samples implanted with 1, 3 and 6 at.\% boron. . . . . . . . . . 109

7.49 GIXRD $\left(2^{\circ}\right)$ spectrum and peak analysis of the AISI 316 sample implanted with 6 at.\%

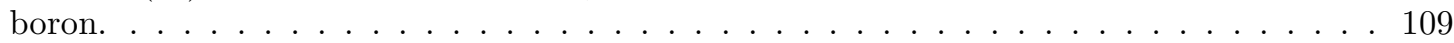

7.50 Polar diagrams of the coercive field $H_{\mathrm{C}}$ (top) and the relative remanence $M_{\mathrm{R}} / M_{\mathrm{S}}$ (bottom) of AISI 316 sample implanted with 1 at.\% boron. . . . . . . . . . . . . . . . . . . . 109 
7.51 Mössbauer spectra of the implanted AISI 316 films. The numbers in the graphs represent the implanted boron concentration. . . . . . . . . . . . . . . . . . . . . . . 110

7.52 Peak position and peak width of the first broad peak in the XRD spectra of the STPLD films. . . . . . . . . . . . . . . . . . . . . . . . 110

7.53 GIXRD $\left(2^{\circ}\right)$ spectra of the carburized films. The stainless steel - boron nitride area fractions are given in the graph. . . . . . . . . . . . . . . . . . . . . . . 111

7.54 Mössbauer spectra of the STPLD stainless steel films. The numbers in the graphs represent the stainless steel/boron-nitride fraction of the STPLD targets. . . . . . . . . . . . 112

7.55 Polar diagrams of the coercive field $H_{\mathrm{C}}$ (top) and the relative remanence $M_{\mathrm{R}} / M_{\mathrm{S}}$ (bottom) of SS/BN 90:10 STPLD sample. . . . . . . . . . . . . . . . . . . . . . . . 113

7.56 RBS spectra of the STPLD films. The stainless steel/boron-nitride area ratios are given in the graph. . . . . . . . . . . . . . . . . . . . . . . 113

7.57 TEM pattern of the SS/BN 80:20 STPLD film. The two numbers in the pattern indicate the position, where the histograms were taken. . . . . . . . . . . . . . 113

7.58 Histograms taken from the positions shown in the SS/bn 80:20 TEM pattern. . . . . . . . 114

8.1 Light microscope images of the FEL surface processed stainless steel samples. Top: AISI 310; bottom AISI 316. . . . . . . . . . . . . . . . . . . . . 117

8.2 GIXRD $\left(2^{\circ}\right)$ spectra of the FEL nitridedfilms. The stainless steel - boron nitride area fractions are given in the graph. . . . . . . . . . . . . . . . . . . . . 117

8.3 Mössbauer spectra of FEL nitrided AISI 310 and AISI 316 samples. . . . . . . . . . . . . 118

8.4 RBS spectra of the FEL nitrided AISI 310 and AISI 316 samples. . . . . . . . . . . . 118

8.5 Hardness-depth profiles of the FEL nitrided AISI 310 and AISI 316 samples. . . . . . . 118 



\section{List of Tables}

2.1 Fe-O crystal structure data. . . . . . . . . . . . . . . . . . . . . . . 24

2.2 Fe-B crystal structure data. . . . . . . . . . . . . . . . . . . . . . . . . . . . . . 25

2.3 Chemical composition of AISI 316. All numeric data are given in wt.\%. . . . . . . . . . 25

4.1 Set of parameter for the FEL surface processing: $\mathrm{P}$ is the power, $f_{p, \text { micro }}$ the frequency of the micro-pulse, $\emptyset_{\text {focus }}$ the diameter of the FEL radiation and $\aleph_{s h, i}$ the track displacement of the laser radiation for steel $i$. . . . . . . . . . . . . . . . . . . . . . 34

4.2 Parameters of the electron beam in the ESRF storage ring . . . . . . . . . . . . 38

4.3 Parameters of all RBS measurements at the IONAS facility in Göttingen . . . . . . . . . . 42

5.1 Fitting results of the MOKE analysis. $\chi^{2}$ and $\chi_{r e d}^{2}$ represent the values of the statistical significance tests. . . . . . . . . . . . . . . . . . . . . .

6.1 Gas flow $j$, deposition time $t$, real film thickness $d$ (as measured by Rutherford Backscattering Spectrometry (RBS) after deposition) and derived growth rate $g=\frac{d}{t}$ for the deposited films. All samples were deposited at $298 \mathrm{~K}$ with a magnetron power of $100 \mathrm{~W}$ and a target-substrate distance of $2 \mathrm{~cm}$. . . . . . . . . . . . . . . . . . .

6.2 Mössbauer fitting results of the sputtered FeC samples, deposited at room temperature with a magnetron power of $100 \mathrm{~W}$ ( $f$ - area fraction (error), mean values of $\langle\delta\rangle$ - isomer shift, $\langle\Delta\rangle$ - the quadrupole splitting for the paramagnetic subspectra, $\langle\epsilon\rangle$ the quadrupole splitting for the magnetic subspectra, $B$ - hyperfine field). . . . . . . . . . .

6.3 Gas flow $j$, deposition time $t$, real film thickness $d$ (as measured by Rutherford Backscattering Spectrometry (RBS) after deposition) and derived growth rate $g=\frac{d}{t}$ for the deposited carburized films. All samples were deposited at $298 \mathrm{~K}$ with a magnetron power of $100 \mathrm{~W}$ and a target-substrate distance of $10 \mathrm{~cm} \ldots \ldots \ldots \ldots \ldots$

6.4 Mössbauer fitting results of the carburized AISI 316 samples, deposited at room temperature with a magnetron power of $100 \mathrm{~W}$ ( $f$ - area fraction (error), mean values of $\langle\delta\rangle$ - isomer shift, $\langle\Delta\rangle$ - the quadrupole splitting for the paramagnetic subspectra, $\langle\epsilon\rangle$ the quadrupole splitting for the magnetic subspectra, $B$ - hyperfine field $). \ldots \ldots \ldots$

6.5 Mössbauer results for the vacuum annealed sample deposited with 0.01 sccm methane flow ( $\delta$ - isomer shift, $\Delta$ - the quadrupole splitting for the paramagnetic subspectra, $\epsilon$ the quadrupole splitting for the magnetic subspectra, $B$ - hyperfine field, $\Gamma$ - line width

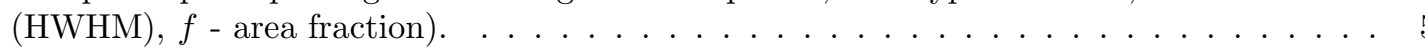

6.6 Raman results of the vacuum-annealed samples . . . . . . . . . . . . . . . . 58

6.7 CEM results of AISI 316 films, deposited at $673 \mathrm{~K}$ with a magnetron power of $100 \mathrm{~W}$ and $0.01 \mathrm{sccm} \mathrm{CH}_{4}$ flow ( $f$ - area fraction (error), mean values of $\delta$ - isomer shift, $\langle\Delta\rangle$ - the quadrupole splitting for the paramagnetic subspectra, $\langle\epsilon\rangle$ the quadrupole splitting for the magnetic subspectra, $B$ - hyperfine field). 
6.8 Hardness, Young modulus and C-content of carburized AISI 310 films, where $E$ is the elastic modulus and $v$ the poisson ratio . . . . . . . . . . . . . . .

6.9 Hardness, Young modulus and C-content of carburized AISI 316 films, where $E$ is the elastic modulus and $v$ the poisson ratio . . . . . . . . . . . . . . . .

6.10 Carbide Formation Ability (CFA) of AISI steels. The lattice constants $a$ are also given. $\quad$ • 62

6.11 Fit results for the Fe k-edge. . . . . . . . . . . . . . . . . . . . . . . . . . 64

6.12 Fit results for the Ni k-edge. . . . . . . . . . . . . . . . . . .

6.13 A list of used alloying elements and selected properties: $\mathrm{r}$ is the atomic radius; $R_{i / j}$ is the the ratio $(R)$ of the solute atom radius to the solvent atom radius; $\mathrm{R}_{N}^{*}$ is the radius ratio, where the subscript $\mathrm{N}$ specifies the particular coordination number; $\sigma^{e^{-}}$is the electronic configuration and CFA the Carbide Formation Ability. . . . . . . . . . . . . .

6.14 Gas flow $j$, deposition time $t$, real film thickness $d$ (as measured by Rutherford Backscattering Spectrometry (RBS) after deposition) and derived growth rate $g=\frac{d}{t}$ for the deposited nitrided films. All samples were deposited at $298 \mathrm{~K}$ with a magnetron power of $100 \mathrm{~W}$ and a target-substrate distance of $10 \mathrm{~cm}$. . . . . . . . . . . . . . . . .

6.15 Mössbauer fitting results of the nitrided AISI 316 samples, deposited at room temperature with a magnetron power of $100 \mathrm{~W}$ ( $f$ - area fraction (error), mean values of $\langle\delta\rangle$ - isomer shift, $\langle\Delta\rangle$ - the quadrupole splitting for the paramagnetic subspectra, $\langle\epsilon\rangle$ the quadrupole splitting for the magnetic subspectra, $B$ - hyperfine field $) \ldots \ldots \ldots \ldots$

6.16 Hyperfine interaction parameters of the singe line analysis of the as-sputtered samples shown in Fig. 6.44 e) and f). Hyperfine parameters: $\delta$ is the isomer shift, QS the quadrupole splitting, $\Gamma$ the line width $(\mathrm{HWHM})$, and RA the relative area. . . . . . . . . .

6.17 PAC interaction parameters of the annealed amorphous and soft ferromagnetic phase at $973 \mathrm{~K}$ for 2 hours shown in Fig. $6.49 \mathrm{~b}$ ). Hyperfine parameters: $\nu_{Q}$ is the quadrupole interaction frequency, $\delta$ the damping of $\nu_{Q}, \eta$ the asymmetry parameter, and RA the relative area. . . . . . . . . . . . . . . . . . . . .

6.18 Hardness, Young modulus and N-content of nitrided AISI 316 films, where $E$ is the elastic

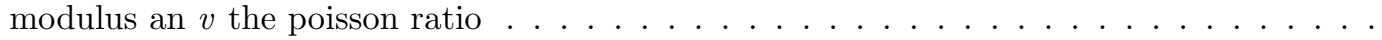

6.19 Gas flow $j$, deposition time $t$, real film thickness $d$ (as measured by Rutherford Backscattering Spectrometry (RBS) after deposition) and derived growth rate $g=\frac{d}{t}$ for the deposited oxidized films. All samples were deposited at $298 \mathrm{~K}$ with a magnetron power of $100 \mathrm{~W}$

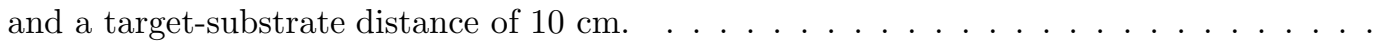

6.20 Mössbauer fitting results of the nitrided AISI 316 samples, deposited at room temperature with a magnetron power of $100 \mathrm{~W}$ ( $f$ - area fraction (error), mean values of $\langle\delta\rangle$ - isomer shift, $\langle\Delta\rangle$ - the quadrupole splitting for the paramagnetic subspectra, $\langle\epsilon\rangle$ the quadrupole splitting for the magnetic subspectra, $B$ - hyperfine field). . . . . . . . . . . .

6.21 PAC interaction parameters of the annealed amorphous and soft ferromagnetic phase at $973 \mathrm{~K}$ for 2 hours shown in Fig. $6.59 \mathrm{~b}$ ). Hyperfine parameters: $\nu_{Q}$ is the quadrupole interaction frequency, $\delta$ the damping of $\nu_{Q}, \eta$ the asymmetry parameter, and RA the relative area. . . . . . . . . . . . . . . . . . . . . .

6.22 Hardness, Young modulus and O-content of oxidized AISI 316 films, where $E$ is the elastic

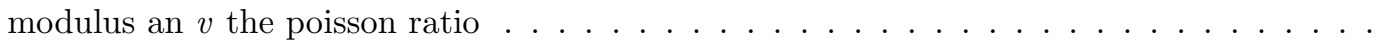

6.23 Summary of the synthesization parameters of the new amorphous and soft ferromagnetic phase: $R$ is the theoretical calculated radius, $R_{I}$ the ionic or van-der-Waals radius, $R_{C}$ the covalent radius, $\sigma_{50: 50}$ the structure of stoichiometric FeX phase (where $X$ represent the reactive gas atom), $\mathrm{T}_{\sigma_{50: 50}}$ the temperature at which the stoichiometric phase can be formed and $\Lambda$ the amorphization parameter. . . . . . . . . . . . . . 
7.1 Deposition temperature $\mathrm{T}_{d e p}$, laser energy, number of pulses \#, real film thickness $d$ (as measured by Rutherford Backscattering Spectrometry (RBS) after deposition) and derived growth rate $g=\frac{d}{t}$ for the deposited STPLD films. . . . . . . . . . . .

7.3 Hyperfine interaction parameters of the singe line analysis of the STPLD FeC film deposited at $823 \mathrm{~K}$. Hyperfine parameters: $\delta$ is the isomer shift, QS the quadrupole splitting, $\Gamma$ the line width $(\mathrm{HWHM})$, and RA the relative area. . . . . . . . . . . .

7.2 Mössbauer fitting results of the STPLD deposited FeC films: ( $f$ - area fraction (error), mean values of $\langle\delta\rangle$ - isomer shift, $\langle\Delta\rangle$ - the quadrupole splitting for the paramagnetic subspectra, $\langle\epsilon\rangle$ the quadrupole splitting for the magnetic subspectra, $B$ - hyperfine field).

7.4 Results of the RBS analysis of the STPLD deposited films.

7.5 Mössbauer fitting results of the RPLD film, deposited at room temperature: ( $f$ - area fraction (error), mean values of $\langle\delta\rangle$ - isomer shift, $\langle\Delta\rangle$ - the quadrupole splitting for the paramagnetic subspectra, $\langle\epsilon\rangle$ the quadrupole splitting for the magnetic subspectra, $B$ hyperfine field $) \ldots \ldots \ldots \ldots \ldots \ldots \ldots$

7.6 Total chamber pressure $j$, deposition time $t$, real film thickness $d$ (as measured by RBS after deposition) and derived growth rate $g=\frac{d}{t}$ for the carburized RPLD films. All samples were deposited at $298 \mathrm{~K}$ with a laser energy of $5 \mathrm{~mJ} / \mathrm{cm}^{2}$ and a target-substrate distance of $7.5 \mathrm{~cm} \ldots \ldots \ldots \ldots \ldots \ldots \ldots$

7.7 Mössbauer fitting results of the RPLD films, deposited at room temperature with a laser energy of $5 \mathrm{~J} / \mathrm{cm}^{2}$ ( $f$ - area fraction (error), mean values of $\langle\delta\rangle$ - isomer shift, $\langle\Delta\rangle$ - the quadrupole splitting for the paramagnetic subspectra, $\langle\epsilon\rangle$ the quadrupole splitting for the magnetic subspectra, $B$ - hyperfine field). . . . . . . . . . . . . .

7.8 Hardness, Young modulus and C-content of RPLD AISI 316 films, where $j$ is the $\mathrm{CH}_{4}$ /chamber pressure, $E$ the elastic modulus an $v$ the poisson ratio. . . . . . . . . .

7.9 Deposition time $t$, real film thickness $d$ (as measured by TEM after deposition) and derived growth rate $g=\frac{d}{t}$ for the STPLD films. All samples were deposited at $298 \mathrm{~K}$ with a laser energy of $5 \mathrm{~mJ} / \mathrm{cm}^{2}$ and a target-substrate distance of $7.5 \mathrm{~cm} . \ldots \ldots$. . . . .

7.10 Mössbauer fitting results of the STPLD stainless steel samples, deposited at room temperature with a laser energy of $5 \mathrm{~J} / \mathrm{cm}^{2}$ ( $f$ - area fraction (error), mean values of $\langle\delta\rangle$ - isomer shift, $\langle\Delta\rangle$ - the quadrupole splitting for the paramagnetic subspectra, $\langle\epsilon\rangle$ the quadrupole splitting for the magnetic subspectra, $B$ - hyperfine field) . . . . . . . . . . . .

7.11 Mössbauer fitting results of the 60:40, 60:40mod, 50:50 and 50:50mod STPLD samples, deposited at room temperature with a laser energy of $5 \mathrm{~J} / \mathrm{cm}^{2}$ ( $f$ - area fraction (error), mean values of $\langle\delta\rangle$ - isomer shift, $\langle\Delta\rangle$ - the quadrupole splitting for the paramagnetic subspectra, $\langle\epsilon\rangle$ the quadrupole splitting for the magnetic subspectra, $B$ - hyperfine field).

7.12 Mössbauer fitting results of the STPLD stainless steel samples, deposited at room temperature $\left(90: 10_{a d}\right)$ and after post-vacuum annealing $\left(90: 10_{p v a}\right): f$ - area fraction (error), mean values of $\langle\delta\rangle$ - isomer shift, $\langle\Delta\rangle$ - the quadrupole splitting for the paramagnetic subspectra, $\langle\epsilon\rangle$ the quadrupole splitting for the magnetic subspectra, $B$ - hyperfine field. . . . . . . .

7.13 Mössbauer fitting results of the 90:10 STPLD stainless steel samples, deposited at room temperature and at $673 \mathrm{~K}(f$ - area fraction (error), mean values of $\langle\delta\rangle$ - isomer shift, $\langle\Delta\rangle$ - the quadrupole splitting for the paramagnetic subspectra, $\langle\epsilon\rangle$ the quadrupole splitting for the magnetic subspectra, $B$ - hyperfine field $) \ldots \ldots \ldots \ldots \ldots$

7.14 Implantation fluences for stainless steel samples containing 1, 3 and 6 at.\% for different implantation energies. The samples are labeled as $1 \mathrm{P}, 3 \mathrm{P}$ and $6 \mathrm{P} \ldots \ldots . . . . . .10$

7.15 Deposition time $t$, real film thickness $d$ (as measured by RBS after deposition) and derived growth rate $g=\frac{d}{t}$ for the STPLD films. All samples were deposited at $298 \mathrm{~K}$ with a laser energy of $5 \mathrm{~mJ} / \mathrm{cm}^{2}$ and a target-substrate distance of $6.5 \mathrm{~cm}$. . . . . . . . . . . . 
7.16 Mössbauer fitting results of the boron implanted AISI 316 samples, deposited at room temperature ( $f$ - area fraction (error), mean values of $\langle\delta\rangle$ - isomer shift, $\langle\Delta\rangle$ - the quadrupole splitting for the paramagnetic subspectra, $\langle\epsilon\rangle$ the quadrupole splitting for the magnetic subspectra, $B$ - hyperfine field $) \ldots \ldots \ldots \ldots \ldots \ldots \ldots$

7.17 Mössbauer fitting results of the boron implanted AISI 316 samples, deposited at room temperature $(f$ - area fraction (error), mean values of $\langle\delta\rangle$ - isomer shift, $\langle\Delta\rangle$ - the quadrupole splitting for the paramagnetic subspectra, $\langle\epsilon\rangle$ the quadrupole splitting for the magnetic subspectra,$B$ - hyperfine field $)$. . . . . . . . . . . . . . . . . . . . . . . 114

8.1 Mössbauer results for FEL nitrided stainless steel samples $(\delta$ - isomer shift, $\Delta-$ the quadrupole splitting for the paramagnetic subspectra, $\epsilon$ the quadrupole splitting for the magnetic subspectra, $B$ - hyperfine field, $\Gamma$ - line width (HWHM), $f$ - area fraction). . . 118 


\section{Chapter 1}

\section{Introduction}

Iron and iron-based alloys have been used by mankind for at least 3,000 years. Its outstanding position in modern society results from its numerous beneficial criteria including the abundance and low cost of iron, its main constituents, manufacturing ability and its recyclability. After several millennia and extensive research, one may think that developments in iron-based alloys are part of the past and its properties have already been developed and exploited to its maximum, but in the last four decades, the development of metastable alloys has attracted the research society: the class of amorphous materials and metallic glasses.

These alloys exhibit a large variety of compositions and provide atomic configurations which are different from their crystalline alloys and reveal various interesting properties, such as good tribological, magnetic and unique chemical properties [1-4].

In Fig. 1.1 the progress in amorphous phase formation is shown, which has finally lead to the development of bulk metallic glasses (BMGs) [5] and which are based on certain late transition metals such as $\mathrm{Pd}, \mathrm{Pt}, \mathrm{Ln}$ (=lanthanide series) and $\mathrm{Zr}$.

In contrast to Fe-based alloys, these new class steels are very expensive due to the huge demand of $\mathrm{Pd}$, $\mathrm{Pt}$ and $\mathrm{Zr}$ by the manufacturing car industry, which led to a price for $\mathrm{Pt}$ and $\mathrm{Pd}$ of approximately $\$ 900.00$ and $\$ 190.00$ an ounce, respectively; and this will not be the end of the line. Stock market predicts a Pd price of $\$ 450.00$ in one year and $\$ 1100.00$ in three years [6]. In opposition, iron costs $\$ 0.53$ an ounce. Thus, the amorphization of Fe-based alloys and the formation of metallic glasses based on conventional steels would be desirable.

Small additions of alloying elements such as boron, oxygen, carbon, or nitrogen, have crucial influence on the deformation behavior, on the mechanical and tribological properties of steel. The modification of the steel properties are originated in strong interactions between interstitial alloying atoms and defects such as vacancies, interstitials and grain boundaries $[7,8]$. Thus, it is necessary to determine in more detail the effects of individual alloying elements on the Fe and steel properties.

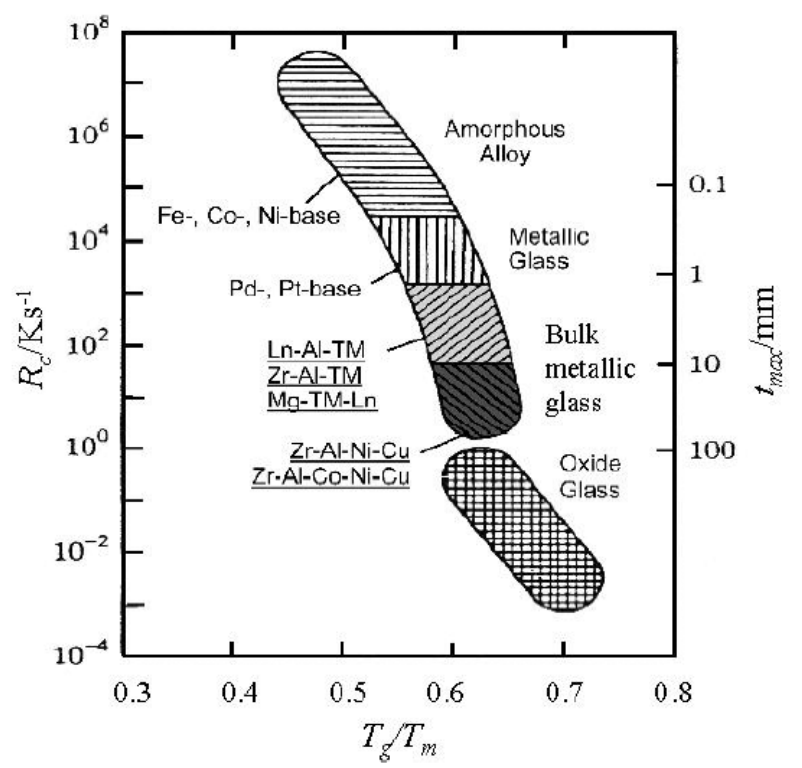

Figure 1.1: Relation between the critical cooling rate $\left(R_{C}\right)$ for glass formation, the maximum sample thickness $\left(t_{\max }\right)$ and the ratio of liquidus and melting temperature $\left(T_{l} / T_{m}\right)[5]$.

One of the more recent works, which attempts to develop a theoretical model of the binary Fe-C/Fe-N system, was published by Byeng-Joo Lee $[9,10]$. By using a modified embedded-atom method (MEAM) [11], which is said to be highly applicable to multicomponent systems, he calculated an interatomic potential for the Fe-C/Fe-N binary system and predicted the physical properties of a hypothetical $\mathrm{NaCl}-$ type $\mathrm{FeC} / \mathrm{FeN}$ phase.

This was the impulse to investigate the Fe-C system for high-carbon concentrations to get a comprehensive sketch of carbide formation and to be able to amorphize conventional steels of the AISI $3 x x$ family and not those late-transition-Fe-based steels as mentioned in the literature [12-14].

As a matter of fact, a cubic FeC phase, which was prepared by means of pulsed laser deposition (PLD), was found and its parameters were found 
very close to those as predicted by Lee. Otherwise, the magnetron-sputtering technique exposed as unsuitable to form cubic $\mathrm{FeC}$ structures and various carbides were observed instead.

However, reactive magnetron-sputtering showed the formation of an amorphous and soft ferromagnetic phase in a wide range of the processing parameters and for different reactive gases (e.g. $\mathrm{CH}_{4}, \mathrm{~N}_{2}$ and $\mathrm{O}_{2}$ ). These films exhibit quasi-metallic glass behavior and the origin and magnitude of magnetism was deduced from a disordered $\mathrm{Ni}_{3} \mathrm{C}$ phase for carburized/nitrided films, and from a disordered $\mathrm{NiFe}_{2} \mathrm{O}_{4}$ for oxidized films.

Also PLD revealed interesting film properties: whereas films synthesized by reactive PLD showed the same physical properties as those synthesized by reactive magnetron-sputtering, inert PLD from precombined targets revealed a self-organized structure, which could be controlled by the processing parameters.

As mentioned at the beginning, iron and steel are still topics in advanced research and were under the focus to develop "high tech" materials with newer and better properties.

In the following chapter 2 , a brief review on the binary phase diagrams of the used systems will be shown. Chapter 3 will give a short introduction to the metallic glass formation by defining the principal conditions to retain an amorphous structure and presenting a theory of metallic glass formation. In Chapter 4, the synthesization of the films (4.1) and the analyzing methods will be discussed. The analyzing methods section is subdivided into three parts: 1) the phase analysis section, where the methods of Mössbauer Spectroscopy, Extended X-ray Absorbtion Fine-Structure, X-ray diffraction and Transmission Electron Microscopy and 2) the chemical composition analysis section, where Rutherford-Backscattering Spectrometry and Resonant Nuclear Reaction Analysis are explained. In the last section 3), other methods such as PAC, MOKE, AFM/STM, Raman spectroscopy and corrosion tests are summarized.

Chapter 5 to 8 will report on the experimental results of the targets and starting materials (chapter 5), the magnetron-sputtered films (chapter 6), the pulsed laser deposited films (chapter 6) and on the laser surface treated samples (chapter 8). The conclusions of the work and the outlooks for future developments and investigations will be drawn in Chapter 9. 


\section{Chapter 2}

\section{Relevant phase diagrams and structures}

The subject of this work is the synthesis of amorphous iron/stainless steel films prepared via magnetron sputtering and pulsed laser deposition. As it will be shown, during magnetron sputtering and pulsed laser deposition, carbon, nitrogen, oxygen and boron are incorporated into the deposited films. As a consequence, the formation of different carbides, nitrides, oxides and borides are observed. Therefor, this section explains the various phase diagrams and properties of the used materials.

\subsection{The Fe-C and Fe-N Sys- tem}

A study of the microstructure of all Fe-based alloys, such as steels, usually starts with the metastable binary Fe-C phase diagram, which is illustrated in Fig. 2.1.

At the low-carbon end of the metastable Fe-C

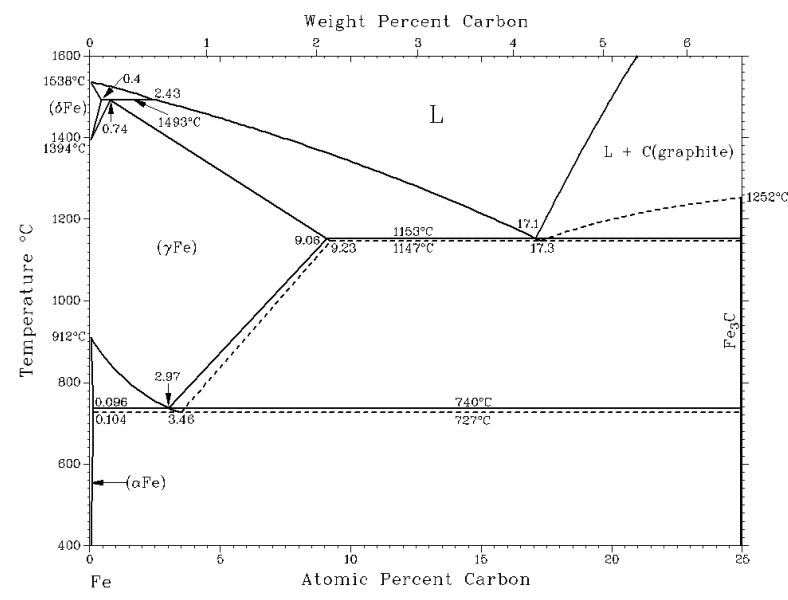

Figure 2.1: The Fe-C phase diagram [15].

phase diagram, one can distinguish ferrite $(\alpha-\mathrm{Fe})$, which can at most dissolve $\sim 0.1$ at. $\% \mathrm{C}$ at $740^{\circ} \mathrm{C}$, and austenite ( $\gamma$-iron), which can dissolve $\sim 9$ at. $\%$ $\mathrm{C}$ at $1154^{\circ} \mathrm{C}$. The much larger phase field of $\gamma$ $\mathrm{Fe}$ (austenite) compared with that of $\alpha$-Fe (ferrite) indicates clearly the considerably grater solubility of carbon in $\gamma$-Fe (austenite). The hardening of carbon containing Fe films, such as carbon steels and many alloy steels, is based on this difference in the solubility of carbon in $\alpha$-Fe (ferrite) and $\gamma$-Fe (austenite).

At the carbon-rich side of the metastable $\mathrm{Fe}-\mathrm{C}$ phase diagram cementite $\left(\theta-\mathrm{Fe}_{3} \mathrm{C}\right)$ can be found. Of less interest, except for highly alloyed steels, is the $\delta$-ferrite at the highest temperatures.

At ambient pressure, body-centered cubic (BCC) ferrite is stable from all temperatures up to $912^{\circ} \mathrm{C}$, when it transforms into face-centered cubic (FCC) austenite. It reverts to ferrite at $1394^{\circ} \mathrm{C}$. This hightemperature ferrite is labeled $\delta$-Fe, even though its crystal structure is identical to that of $\alpha$ ferrite. The $\delta$-ferrite remains stable until it melts at $1538^{\circ} \mathrm{C}$.

Also other metastable phases, such as $\epsilon / \theta-\mathrm{Fe}_{2} \mathrm{C}, \chi-$ $\mathrm{Fe}_{5} \mathrm{C}_{2}, \mathrm{Fe}_{7} \mathrm{C}_{3}, \mathrm{Fe}_{2} 0 \mathrm{C}_{9}$ and $\mathrm{Fe}_{2} 3 \mathrm{C}_{6}$ have been reported [16, 17].

Regions with mixtures of two phases (such as ferrite+cementite, austenite+cementite, and ferrite+austenite) are found between the single-phase fields. At the highest temperatures, the liquid phase field can be found, and below this are the twophase fields (liquid+austenite, liquid+cementite, and liquid $+\delta$-ferrite).

Similar to carbon, nitrogen also occupies interstitial sites in the Fe lattice. There are five equilibrium solid phases in the Fe-N system, which is shown in Fig. 2.2 [15].

The phases are: $\alpha-\mathrm{Fe}(\mathrm{N}), \gamma-\mathrm{Fe}(\mathrm{N}), \gamma^{\prime}-\mathrm{Fe}_{4} \mathrm{~N}, \zeta-$ $\mathrm{Fe}_{2} \mathrm{~N}$ and $\epsilon-\mathrm{Fe}_{3} \mathrm{~N}$. In the $\alpha$-Fe, the maximum nitrogen solubility is $\sim 0.4$ at. $\%$ at $592^{\circ} \mathrm{C}$, but it can reach $\sim 10.3$ at. $\%$ at $650^{\circ} \mathrm{C}$ in the $\gamma$-Fe. The stoichiometric $\gamma^{\prime}-\mathrm{Fe}_{4} \mathrm{~N}$ phase exists in a narrow re- 


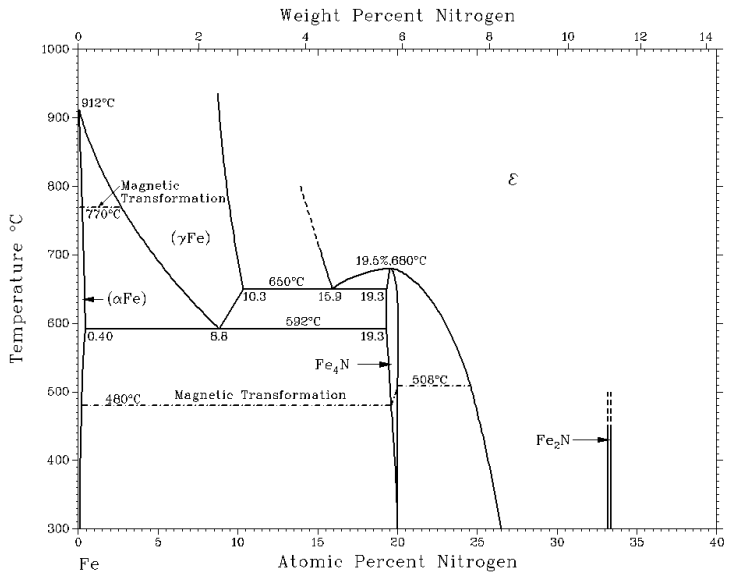

Figure 2.2: The Fe-N phase diagram [15].

gion that extends up to $680^{\circ} \mathrm{C}$ and crystallizes in a cubic structure. The hexagonal $\epsilon-\mathrm{Fe}_{x} \mathrm{~N}$ phase is stable and crystallizes with structures similar to the $\epsilon-\mathrm{Fe}_{x} \mathrm{C}$ carbide in a large compositional range. The metastable $\alpha "-\mathrm{Fe}_{16} \mathrm{~N}_{2}$ and the body-centered tetragonal martensite phases has been reported as well [17].

\subsection{The Fe-O System}

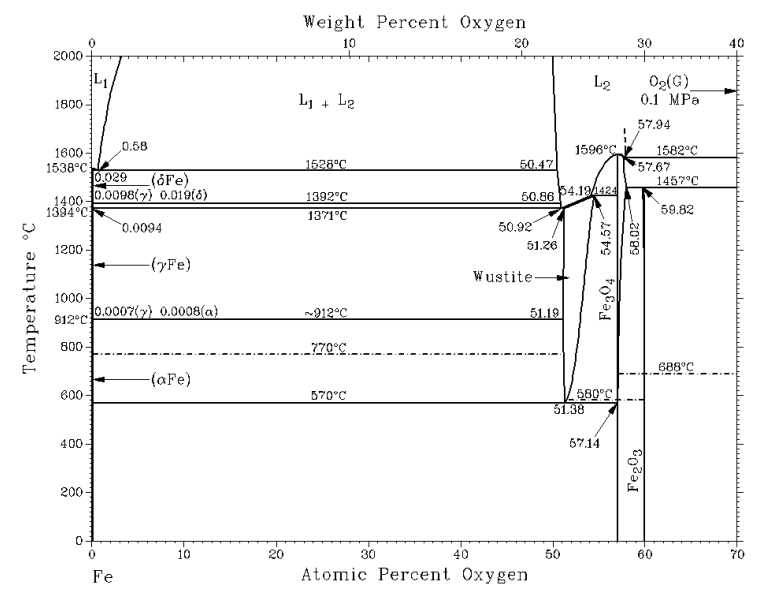

Figure 2.3: The Fe-O phase diagram [15].

The stable Fe-O system consists of five solid phases [15]: (1) the terminal BCC solid solution with a narrow range of composition denoted as $\alpha-\mathrm{Fe}(\mathrm{O})$ or $\delta$ - $\mathrm{Fe}(\mathrm{O})$, with the temperature designations used below $912^{\circ} \mathrm{C}$ and above $1394^{\circ} \mathrm{C}$, respectively; (2) the terminal FCC solution denoted as austenite or $\gamma$-Fe, with a narrow range of composition extending approximately from $912^{\circ} \mathrm{C}$ to $1394^{\circ} \mathrm{C}$, the stable temperature range of $\gamma$-Fe; $(3)$ the FCC oxides, denoted as $\mathrm{FeO}, \mathrm{Fe}_{1-x} \mathrm{O}, \mathrm{Fe}_{x} \mathrm{O}, \mathrm{FeO}_{1+x}$ or $\mathrm{FeO}_{x}$, wustite, with broad range of compositions, which may possibly can be subdivided into regions of differing types or degrees of order; (4) $\mathrm{Fe}_{3} \mathrm{O}_{4}$ denoted as magnetite, which is monoclitic and almost stoichiometric below $-149^{\circ} \mathrm{C}$ and is FCC above $-149^{\circ} \mathrm{C}$, with a range of compositions largely broadened at high temperatures; and (5) the rhombohedral oxide $\mathrm{Fe}_{2} \mathrm{O}_{3}$, called hematite, which is almost stoichiometric at low temperatures, but has an excessive broadened range of compositions at high temperatures.

The most important phases of the Fe-O phase diagram (Fig. 2.3) and its parameters can be found in Table 2.1.

Table 2.1: Fe-O crystal structure data.

\begin{tabular}{lccc}
\hline Phase & $\begin{array}{c}\text { Composition } \\
\text { [at\% O] }\end{array}$ & $\begin{array}{c}\text { Space } \\
\text { group }\end{array}$ & $\begin{array}{c}\text { lattice } \\
\text { constant } \\
\text { [nm] }\end{array}$ \\
\hline Stable phases & $\sim 0$ & $\operatorname{Im} \overline{3} m$ & 0.2866 \\
$\alpha$-Fe & $\sim 0$ & $\operatorname{Im} \overline{3} m$ & 0.2866 \\
$\delta$-Fe & $\sim 0$ & $F m \overline{3} m$ & 0.3590 \\
$\gamma$-Fe & 51.2 to 54.7 & $F m \overline{3} m$ & 0.4307 \\
wustite & $\sim 57.1$ & $C c$ & $\ldots$ \\
$\mathrm{Fe}_{3} \mathrm{O}_{4}(\mathrm{LT})$ & 57.1 to 58.0 & $F d \overline{3} m$ & 0.8396 \\
$\mathrm{Fe}_{3} \mathrm{O}_{4}$ & $\sim 60.0$ & $R \overline{3} c$ & a: 0.5035 \\
$\alpha-\mathrm{Fe}_{2} \mathrm{O}_{3}$ & & & c: 1.3740 \\
$\mathrm{Metastable}_{\text {phases }}$ & & & \\
$\beta-\mathrm{Fe}_{2} \mathrm{O}_{3}$ & $\sim 60.0$ & $\operatorname{Ia} \overline{3}$ & 0.9404 \\
$\gamma-\mathrm{Fe}_{2} \mathrm{O}_{3}$ & $\sim 60.0$ & $P 432_{1} 2$ & a: 1.0244 \\
& & & b: 1.0534 \\
& & & c: 0.3031 \\
$\epsilon-\mathrm{Fe}_{2} \mathrm{O}_{3}$ & $\sim 60.0$ & $\ldots$ & $\ldots$ \\
\hline
\end{tabular}

\subsection{The Fe-B System}

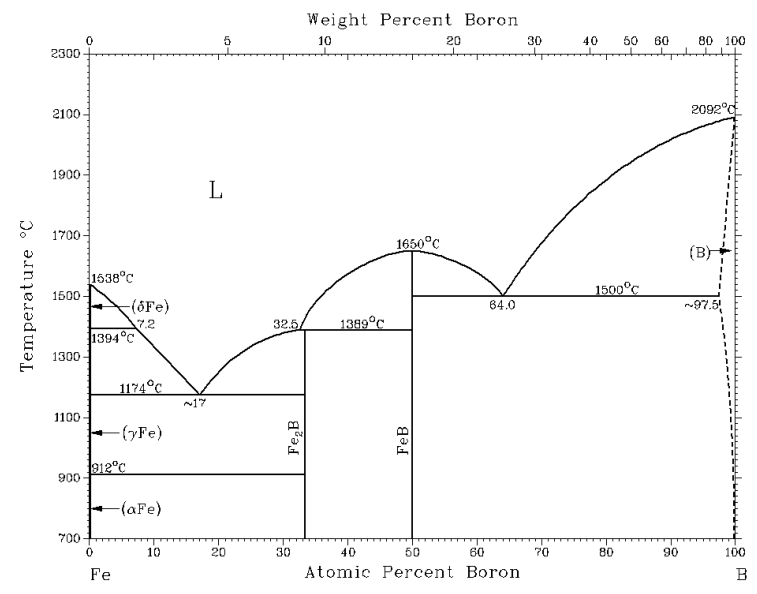

Figure 2.4: The Fe-B phase diagram [15].

The equilibrium of the Fe-B system consists of three phases [15]: (1) the liquid, L; (2) the terminal solid 
solutions - high-temperature BCC $(\delta$-Fe), mediumtemperature FCC $(\gamma-\mathrm{Fe})$, low-temperature BCC $(\alpha$ $\mathrm{Fe})$, and rhombohedral $(\beta-\mathrm{B})$ - characterized by small mutual solid solubility in the elements; and (3) two intermetallic compounds $-\mathrm{Fe}_{2} \mathrm{~B}$ and $\mathrm{FeB}$. The Fe-B system is shown in Fig. 2.4 and summarized in Table 2.2 .

Table 2.2: Fe-B crystal structure data.

\begin{tabular}{|c|c|c|c|}
\hline Phase & $\begin{array}{c}\text { Composition } \\
{[\text { at\% Fe] }}\end{array}$ & $\begin{array}{l}\text { Space } \\
\text { group }\end{array}$ & $\begin{array}{c}\text { lattice } \\
\text { constant } \\
{[\mathrm{nm}]}\end{array}$ \\
\hline Stable phases & & & \\
\hline $\begin{array}{l}\alpha-\mathrm{Fe} \\
\mathrm{Fe}_{2} \mathrm{~B}\end{array}$ & $\begin{array}{c}\sim 0 \\
33.3\end{array}$ & $\begin{array}{l}\operatorname{Im} \overline{3} m \\
I 4 / m c m\end{array}$ & $\begin{array}{c}0.2866 \\
\text { a: } 0.5099 \\
\text { c: } 0.4240\end{array}$ \\
\hline $\mathrm{FeB}$ & 49.5 to 50.0 & Pbmn & $\begin{array}{ll}\text { a: } & 0.4503 \\
\text { b: } & 0.5495 \\
\text { c: } & 0.2946\end{array}$ \\
\hline $\begin{array}{l}\text { Metastable } \\
\text { phases } \\
\mathrm{Fe}_{3} \mathrm{~B}\end{array}$ & $\sim 25.0$ & Pnma & $\begin{array}{ll}\text { a: } & 0.6726 \\
\text { b: } & 0.4331 \\
\text { c: } & 0.5468\end{array}$ \\
\hline
\end{tabular}

\subsection{The Fe-Cr-Ni System}

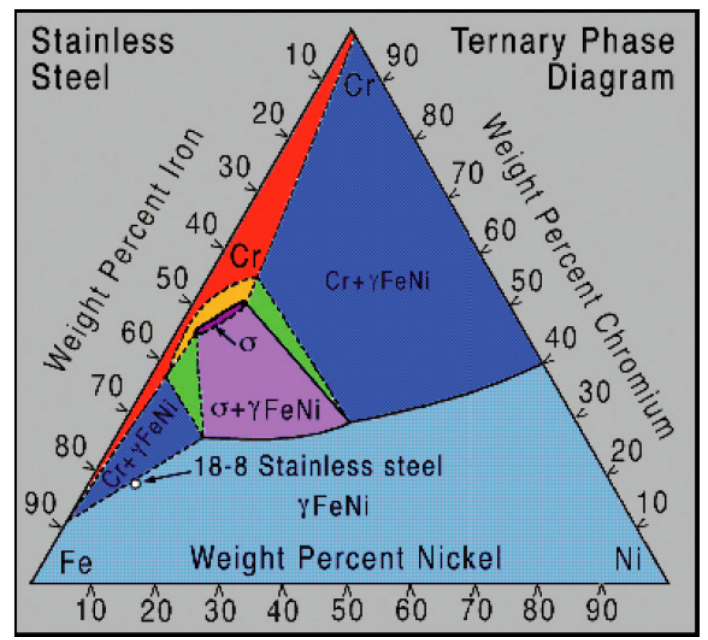

Figure 2.5: Fe-Cr-Ni phase diagram at $900^{\circ} \mathrm{C}$ [18].

In metallurgy, stainless steel is defined as a steel alloy with a minimum of 11.5 wt.\% $\mathrm{Cr}$ content [19]. Higher Cr contents and other alloying elements such as $\mathrm{Ni}$ and $\mathrm{Mo}$ increase the corrosion resistance of these steel. The most important factor for corrosion resistance is known in the $\mathrm{Cr}$ content in the film due to the formation of Cr-rich oxides on the surface of the steel. This oxide/passive layer separates the material from any medium and constrains processes such as mass transport, transpassive reactions and chemical conversions, which are involved in the corrosion process.

There are four different stainless steels: austenitic, ferritic, martensitic and combined austeniticferrictic steels.

In this work, AISI 316 is used, its composition can be obtained in Table 2.3. It is an austenitic stainless

Table 2.3: Chemical composition of AISI 316. All numeric data are given in wt.\%.

\begin{tabular}{cccccc}
$\mathrm{C}$ & $\mathrm{Si}$ & $\mathrm{Mn}$ & $\mathrm{Cr}$ & $\mathrm{Mo}$ & $\mathrm{Ni}$ \\
$\leq 0.07$ & $\leq 1.0$ & $\leq 2.0$ & $16.5-18.5$ & $2.0-2.5$ & $10.0-13.0$ \\
\hline
\end{tabular}

steel, antiferromagnetic and its Neel temperature is $-149.5^{\circ} \mathrm{C}[20]$. Its FCC structure is influenced by its $\mathrm{Ni}$ content, which exceeds the $\gamma$-stabilizing Ni content of 8 at. $\%[19,21]$. The ternary phase diagram of the $\mathrm{Fe}-\mathrm{Cr}-\mathrm{Ni}$ compound can be seen in 2.5. 



\section{Chapter 3}

\section{Amorphization of metals}

In the last decades it has become clear, that nanoand non-crystalline alloys can exhibit many potential advantages to their equivalent or similar crystalline phases, such as higher strength and hardness, better magnetic properties, better corrosion resistance and low material costs $[4,14,22]$. This led to a new group of materials: bulk amorphous alloys (also known as BMGs: bulk metallic glasses).

This chapter will give a short introduction to the metallic glass formation by defining the principal conditions to retain an amorphous structure and presenting a theory of metallic glass formation.

\subsection{Metallic glass formation}

The term metallic glass refers to a non-crystalline solid phase and represents the ultimate state of solid metastability and can be obtained from solidification of a liquid, from the vapor phase, deposition from a chemical solution or an electrolyte and by high-energy ion- or neutron bombardment of crystalline materials are also included [3]. In its original sense it is formed by continuous cooling of a liquid. A glass lacks three-dimensional atomic periodicity beyond a few atomic distances and show diffuse halos in X-ray, electron and neutron diffraction and no sharp diffraction contrast in high-resolution electron microscopy. Different approaches have been made to give a physical definition of metallic glasses. The first one concentrates on carefully chosen experimental observations - the phenomenological approach - whereas the second regards the construction of atomistic models - the theoretical approach.

The first approach of a metallic glass defines a vitrificated melt as a frozen liquid, or at least largely so [23]. Some alloys, with specific ranges in their composition, can be cooled down below the melting temperature $T_{m}$. This effect is called undercooling of the melt. The heterogeneous nucleants of the alloy were increasingly delayed for kinetic reasons, whereas the recalescence decreases by en-

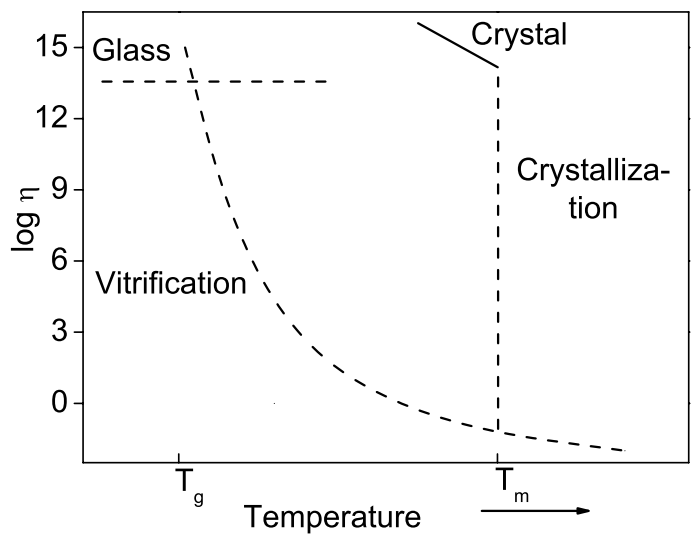

Figure 3.1: Viscosity $(\eta)$-temperature $(T)$ dependence corresponding to crystallization and vitrification of a metallic melt.

hancing the undercooling which finally leads to a steady suppression of crystallization. Initially, this allows a refinement of microstructure and ends in the extension of solute solubility and in the formation of metastable phases. If the cooling rate is high enough, crystallization can be suppressed due to insufficient nucleation, which finally leads to a continuously increase of the viscosity of the melt. This is illustrated in Fig. 3.1.

At the so-called glass transition temperature $T_{g}$, the atomic configuration becomes homogeneously frozen, after starting from the equilibrium state. This structurally freezing to glass state is defined at a viscosity of $10^{13}$ Poise and is the so-called kinetic arrest and is highly cooling-rate dependent. At $T_{g}$, the volume-enthalpy-temperature dependency decrease non-discretely, which can be seen in Fig 3.2. During vitrification, the change in the specific heat of the undercooled liquid increases with falling temperature, as well as the crystalline state. According to the free volume model of atomic transport [24], glass transition corresponds to the absence of free volume.

The second definition of a glass is reported by 


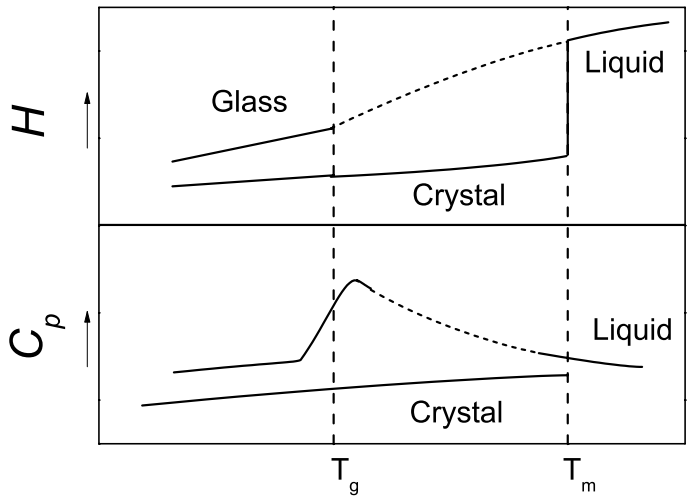

Figure 3.2: Temperature dependence of enthalpy $H$ and specific heat $C_{p}$ corresponding to crystallization and vitrification of a metallic melt.

Cargill et al. [25], in which the glass is described by a combination of an experimental determined radial distribution function (RDF) and of a theoretical RDF of a dense random packing (DRP) model proposed by Bernal [26].

The RDF is defined by:

$$
\varrho(r)=\sum_{R} \frac{n R}{n} \partial(r-R)
$$

where $r$ is the vector between two atoms. It can be obtained by X-ray or neutron diffraction measurements or NMR spectroscopy and gives information about the nearest and next-nearest neighbour atoms lying in a spherical coordination shell as shown in Fig. 3.3 [27].

The DRP is considered to be the first realistic model of liquid structure. The model demonstrates that an aperiodic structure with a physical density comparable to that of a crystal by physically packing many small spheres inside a large spherical volume. Bernal then studied the local topology of several atomic configurations which were unseen in crystalline structures.

The absence of crystallization upon undercooling is explained on the basis of a standard nucleation theory [28]. In this theory, a homogenous nucleation mechanism, which is responsible for the formation of the solid phase, is considered to be hindered by a large energy barrier. This barrier was approved by Frank [29] and is originated in the development of local short-range order structures (mostly with an icosahedral form) of a metallic undercooled melt, which are incompatible with long-range periodicity. As a consequence, several attempts were made to confirm this model, but not till Kelton et al. in 2003 were demonstrating the first experimental evidence between the local order of the liquid and the nucleation barrier upon undercooling [30].

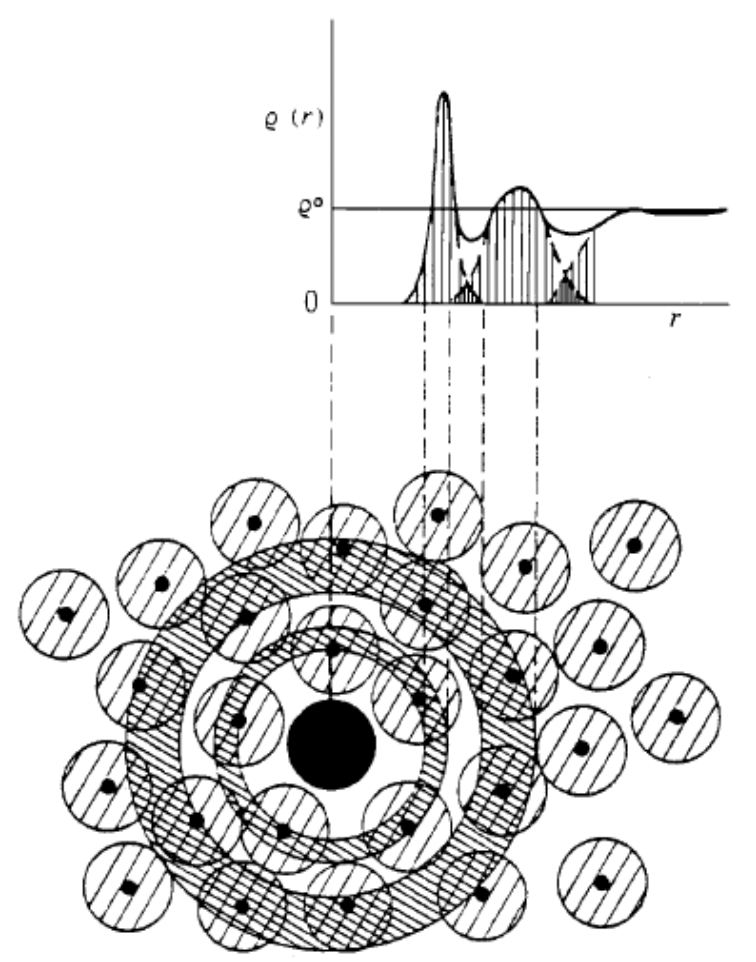

Figure 3.3: Illustration of atomic arrangements in an amorphous solid and the corresponding radial distribution function $\varrho(r)$.

\subsection{Theory of metallic glass formation}

An approach to determine the so-called glass forming ability (GFA) of a system was proposed by Inoue et al. [31]. Inoue suggested the following rules for the GFA of an alloy:

1. A good metallic glass former is a multicomponent system with more than three elements.

2. The alloying elements must have a relative ratio of the atomic radii larger than $12 \%$, which provides dense packing of different elements and, thus, atomic rearrangement can be hindered.

3. The constituents must have a large negative heat of mixing, which is an indication of the tendency of two chemical species to combine and to form a metallic compound. On the contrary, if the enthalpy of mixing is positive the tendency of mutual avoidance is enhanced and the alloy may show phase separations.

An alloy which satisfy Inoue's rules should have a large GFA. These rules can be discussed in terms of 
thermodynamics, kinetics and structural point of views.

From thermodynamics, a large GFA is obtained under the condition of low Gibbs free energy $\Delta G(T)$ for the transformation from the liquid to the solid phase. These low values can be obtained, if the values for enthalpy $\Delta H_{f}$ and entropy $\Delta S_{f}$ of fusion in the Gibbs relation

$$
\Delta G=\Delta H_{f}-T \Delta S_{f}
$$

have its minimum. In multicomponent alloy systems, $\Delta S_{f}$ is expected to be large. An increase of $\Delta S_{f}$ causes an increase of the DRP which is also favorable for a decrease of $\Delta H_{f}$ and an increase of the solid/liquid interfacial energy.

From kinetics, Cohen and Turnbull [32] derived a relation between the rate of homogeneous nucleation frequency, $I_{v}$, and the reduced undercooling temperature $\Delta T_{r}=\left(T_{l}-T\right) / T_{l}$ as follows:

$$
I_{v}=\frac{k_{n}}{\eta(T)} \exp \left(-16 \pi \alpha^{3} \beta / 3 T_{r} \Delta T_{r}^{2}\right)
$$

where $k_{n}$ is a kinetic constant, $\eta(T)$ the shear viscosity at temperature $T$ (which is inversely related to the atomic diffusivity $D$ ) and $T_{r}$ the reduced temperature $T / T_{l}$. Respectively, $\alpha$ and $\beta$ are dimensionless parameters and are related to the liquid/crystal interfacial energy $\sigma$ and to $\Delta S_{f}$, thus:

$$
\begin{aligned}
& \alpha=\left(N \bar{V}^{2}\right)^{1 / 3} \sigma / \Delta H_{f} \\
& \beta=\Delta S_{f} / R
\end{aligned}
$$

where $N$ is the Avogadro's number and $\bar{V}$ the molar volume of the crystal. From the equation for $I_{v}$ it is evident for given temperature and viscosity of a melt that $I_{v}$ decreases precipitously as the magnitude of $\alpha \beta^{1 / 3}$ increases and, thus for high temperatures, $I_{v}$ becomes dominated by the reduced glass temperature $T_{r g}$ as indicated in Fig. 3.1.

As $T_{r g}$ is increased, $\eta$ is increases rapidly with $\Delta T_{r}$. As a consequence, the $I_{v}-T_{r}$ peak is rapidly lowered and shifted to higher $T_{r}$. Thus, nucleation can be avoided easier and the volume in which a single nucleation can be avoided increases. For alloys with $T_{r g}=0.5$, droplets would vitrify with a diameter of about $60 \mu \mathrm{m}$ [3] for cooling rates of $10^{6} \mathrm{~K} / \mathrm{s}$.

A related kinetic approach was taken by Uhlmann [33] and was generalized for all metallic systems by Davies et al. [34]. According to the JohnsonMehl-Avrami treatment of transformation kinetics $[35,36]$, the fraction of a transformed phase $x$ in time $t$ is given by:

$$
x \sim \pi I_{v} u_{c}^{3} t^{4} / 3
$$

where $u_{c}$ is the crystal growth velocity and can expressed as:

$$
u_{c}=\frac{k T_{f}}{3 \pi a_{0}^{2} \eta}\left[1-\exp \left(-\Delta T_{r} \Delta H_{f} / R T\right)\right]
$$

$\mathrm{f}$ is the fraction of sites at the crystal surfaces where atomic attachment can occur (= 1 for close packed crystals and $0.2 \Delta T_{r}$ for faceted crystals) and $a_{0}$ the mean atomic diameter.

In contrast to the kinetic model mentioned above, wherein $I_{v}$ was based on the Hoffman model for free enthalpy of crystallization $\left(\Delta G=T_{r} \Delta T_{r} \Delta H_{f}\right)$, here $I_{v}$ is given by:

$$
I_{v}=\frac{k T \overline{N_{v}}}{3 \pi a_{0}^{3} \eta} \cdot \exp \left(\frac{-1.07}{\Delta T_{r}^{2} T_{r}^{3}}\right)
$$

The pre-exponential constants in equations 3.6 and 3.7 are derived on the assumption that the atomic diffusivity is identical to cross the liquidnucleus and liquid-crystal interfaces and related to $\eta$ through the Stokes-Einstein equation. $\overline{N_{v}}$ is the average atomic volume concentration.

From a structural point of view, the presence of elements with different atomic sizes (about 12\%) in a multicomponent system which have large negative heats of mixing causes an increase of RDP in the undercooled liquid. As a consequence, a large liquid/solid interfacial energy is achieved and is hindering an atomic arrangement of the constituent elements on a long-range scale which suppresses crystalline phase nucleation and crystal growth, respectively [37-39]. 



\section{Chapter 4}

\section{Deposition of thin films and experimental methods}

\subsection{Film Deposition}

In this section, the synthesization techniques of the deposited films were discussed, which were fabricated by magnetron sputtering and pulsed laser deposition. In this work, a planar magnetron is used which is equipped with a radio-frequency bias generator (Plasma Products RF5S, $13.56 \mathrm{MHz}$ ). The PLD chamber was self-assembled as described in 3.1.2 and using a Siemens XP2020 XeCl excimerand a Quantel Brilliant Nd:Yag laser system.

\subsubsection{Magnetron sputtering}

The sputtering phenomenon has been known since 1852 and exploited for deposition of films and is now one of the most extensively used techniques for the deposition of films and coatings. Sputter deposition methods used today have a common simple processing aim: to generate and maintain a desired plasma and to establish a bias or electric field for the acceleration of ions to the electrode or to the target which is then being bombarded.

\section{The principle setup of a planar magnetron}

The magnetron target is based on the work carried out by Penning more than 70 years ago [40] and a lot of work had been done to develop this concept since then. Today, the planar magnetron is the most widely used target assembly, but it was not introduced until the early 1970s by Chapin [41]. Sputter deposition of thin films as functional and protective layers has increased in the last decade which can be ascribed to the development of highperformance magnetron cathodes. In comparison to conventional diode sputtering, the magnetron cathode provides higher deposition rates at a lower operating pressure accompanied by an increased quality

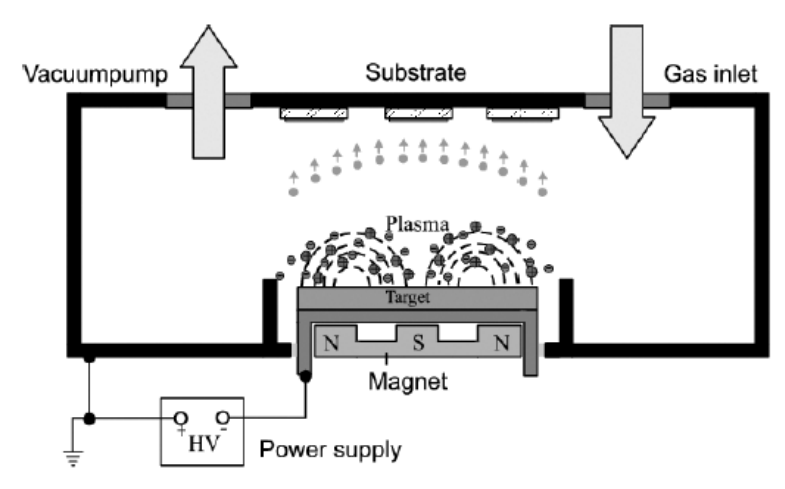

Figure 4.1: Principle setup of a planar magnetron system.

of the films (better adherence and greater uniformity over large areas).

The principle setup of planar Magnetron in its simplest form is shown schematically in Figure 4.1 [42]. It consists of the target material backed by permanent magnets that provide a toroidal confinement field with the field lines forming a closed tunnel on the target surface. The field strength is chosen to provide effective confinement for electrons while allowing heavier ions considerable freedom.

Secondary electrons were emitted from the target during the sputtering process. These are accelerated across the cathode dark space towards the highly charged plasma, which has the form of the so-called Debye sphere. The propagation of the electrons is affected by the Lorentz force and one component of their motion is a helical path about the magnetic field lines. The electrons which propagate along these helical lines in the middle of the target are reflected due to the higher density of field lines and were affected by a repulsive electric field. After reflection the electrons eventually reach the perimeter of the target where the field lines again intersect the surface. An anode placed in this re- 
gion effectively collects these electrons and prevents a drift of the electrons to the substrate. A second component of their propagation is a drift from one field line to another resulting in a toroidal tunnel on the surface of the target. The combined motion gives an extended path length resulting in a large number of collisions of the electrons with gas atoms. The ions which are affected by the same Lorentz force as the electrons were - de to their higher mass - not so hardly influenced in their resulting propagation to the substrate.

\section{Reactive sputtering}

In contrary to inert sputtering, reactive sputtering is identified with the process, where neutral, excited, or ionized gaseous species react with the sputtered particles from the target or with particles at the substrate [43]. Thus, a variety of thin films and compounds, such as oxides, nitrides, and carbides etc. can be deposited by introducing a mixture of reactive gas (e.g. $\mathrm{O}_{2}, \mathrm{~N}_{2}$, or $\mathrm{CH}_{4}$ etc.) and inert gas (e.g. Ar or He) with desired stoichiometries (depending on the processing parameters) can be formed. The mechanism of the sputtering gas interaction is not fully understood, but different models have been introduced. In Figure 4.2 the common mechanism model of L. Holland is shown [44].

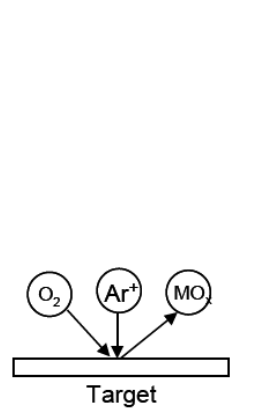

(a)

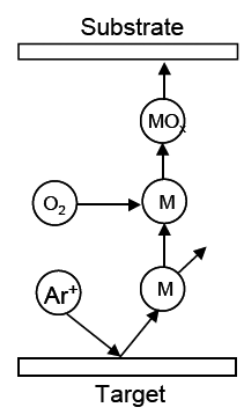

(b)

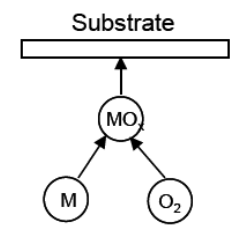

(c)

Figure 4.2: Three different mechanisms for reactive sputter deposition: (a) at the target, (b) in the plasma volume, (c) at the substrate.

\subsubsection{Pulsed Laser Deposition (PLD)}

Pulsed Laser Deposition (PLD) is conceptually and experimentally a relatively simple technique and is enjoying great popularity in the last years. The laser radiation hits direct the target located in a vacuum chamber at an inclination angle of e.g. $45^{\circ}$ with respect to the normal of a substrate-holder. The impact of the laser radiation on the target surface results in various processes including melting, evaporation, and ablation of the target material with production of a plasma due to excitation and ionization of the species ejected from the target by the laser radiation. All these processes are triggered by conversion of the optical energy into thermal, chemical, and mechanical energy. The ablated material is deposited on a substrate positioned on a heatable substrate holder.

The principle setup of the used PLD system is displayed in Fig. 4.3.

During deposition, a fine control of the film thickness can be achieved by the number of pulses. Thus, a fast response in exploiting new material systems is a unique feature of PLD among other deposition techniques. The most important feature of PLD is that the stoichiometry of the target can be retained in the deposited films. The extremely high heating rate of the target surface $\left(10^{8} \mathrm{~K} / \mathrm{s}\right)$ caused by the pulsed laser irradiation leads to the congruent evaporation of compounds from the target irrespective of the sublimation point of the target constituent elements.

In spite of the advantages of PLD, some drawbacks have been identified in using this technique. One of the major restriction is the formation of droplets due to splashings from the target. The size of the droplets are usually a few microns. In general, such droplets have to be avoided otherwise they greatly affect the growth as well as the properties of the films. Another restriction is the narrow angular distribution of the species, which are generated by the adiabatic expansion of the laserinduced plasma. In general some solutions have been proposed, such as the inserting a shadow mask to block off the particulate and rotating both target and substrate in order to produce uniform films. On the other hands, the features previously mentioned limit the use of PLD in producing large area thin films that PLD has not yet been fully deployed in industry. Recently remedial measures, in order to produce a larger uniform film, have been developed to minimize these restrictions in PLD, for instance, the simultaneous movements of target and substrates (using a robotic/automated/ positioning mechanisms) in combination with the use of certain power density distribution yielding a special angular distribution of the laser induced vapourplasma. In addition, various targets may be used to increase the versatility of laser radiation to deposit large area thin films. 

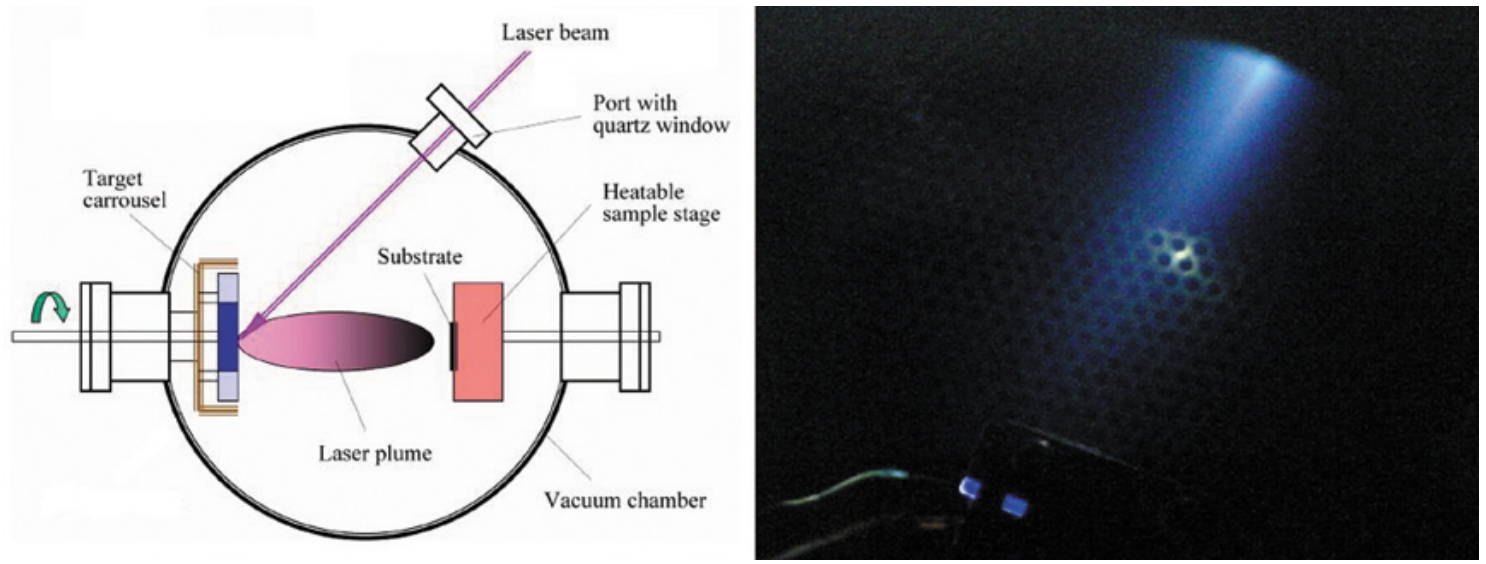

Figure 4.3: Principle setup of the PLD system (left) and typical PLD plasma (right).

\subsubsection{Description of physical phenomena during PLD processing}

The vaporisation threshold, viz. the minimum laser intensity necessary for bulk material evaporation can be described by the equation:

$$
I_{\text {vap }}=\frac{c_{P} \rho \sqrt{\pi \chi}\left(T_{\text {vap }}-T_{0}\right)}{2(1-R) \sqrt{\tau_{\text {las }}}}
$$

where $\mathrm{C}_{p}, \rho$ and $\chi$ are the heat capacity, specific mass, and the thermal diffusivity of the target material, $R$ the reflectivity of the surface of the target, $T_{\text {vap }}$ the vaporization temperature and $T_{0}$ the ambient temperature. Note, this formula is only valid for a laser pulse with a rectangular temporal shape of duration $\tau_{\text {las }}$.

To get thin layers of high quality, particle/droplet formation has to be avoided. Two main factors for particle/droplet formation during laser evaporation are: 1) the separation of surface defects under thermal shock and 2) splashing of liquid material due to superheating of subsurface layers, which appears for fast heating rates. To avoid superheating, the laser intensity must be lower than that intensity, which is needed to evaporate a mass volume with a depth of $\delta_{t h} / 2$ (the heat diffusion length is defined as $\delta_{t h}=\sqrt{2 \chi t}$ ). This gives an upper limit for laser intensity to be applied in a PLD process. The superheating threshold is given by [45]

$$
I_{t h}=\frac{\rho \Delta H_{v a p}}{\alpha t_{r}}
$$

where $\alpha$ is the absorption coefficient, $t_{r}$ the relaxation time of hot electrons on the surface, $\Delta H_{\text {vap }}$ the enthalpy change per mass unit including latent heat for fusion and vaporization and heating from ambient temperature to the vaporization point.

For laser intensities slightly lower than the vaporization threshold, very low material sputtering rates in vacuum result in a collision less than low density vapor in front of the target surface. Evaporation appears to be a thermal process. When the laser intensity increases, the vapor density rises and collisions occur in a zone onto the surface. This is the so-called Knudsen layer. Increasing the laser intensity leads to an increase of atom density and temperature in the Knudsen layer. The ionization degree rises. By increasing the laser intensity above the plasma ignition threshold, a fully ionized vapor plasma can be identified.

Laser heating of metals can be modeled by three basic processes: 1) the deposition of radiation energy on free electrons, 2) the energy exchange between electrons and lattice, and 3) the propagation of energy through media by free electron motion $[45,46]$. In the course of high power laser heating, where the metal is looked upon as a two-temperature system, the free electrons are heated to an effective temperature much higher than that of the lattice. As a result, large local temperature gradients arise between the electrons and the lattice. Subsequently, the transport of energy from the electrons to the lattice takes place by means of a relaxation mechanism and a heat transfer coupling coefficient between electron and phonon subsystem is considered.

\subsection{Free Electron Laser sur- face processing}

To compare the properties of thin films to those of surface treated films, Free Electron Laser (FEL) surface processing experiments at the Jefferson Lab were carried out. As well as synchrotron facilities, FELs were used in bioscience, chemistry, solid-state physics, advanced materials and basic research (also manufacturing, medical and military purposes are 


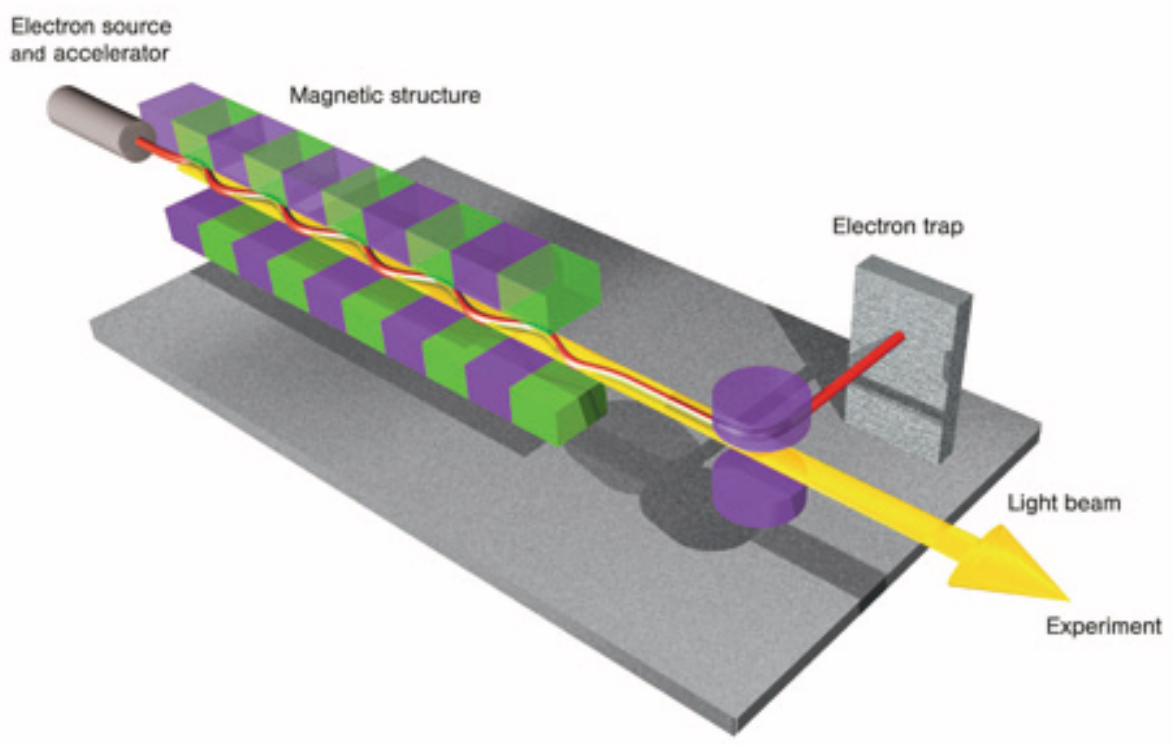

Figure 4.4: Principle setup of the FEL system used at the Jefferson lab.

planned) due to their high-rated potential. These advantages include amongst others high peak and average power, bandwidth, beam quality and pulse brevity.

The main advantage of FELs are their ability to be tunable over a wide range of wavelengths, which opens their usage not only to basic research facilities. This tunability results from a basic difference between FELs to conventional lasers: whereas conventional lasers use electrons bounded in atoms or molecules, the FEL uses an beam of unbounded electron, which is accelerated nearly to speed of light. Using a magnetic device - called undulator - the electrons were conducted on a sinusoidal orbit. They are above the ground state and represent a population inversion. In the undulator the electron yield synchrotron radiation, which is shown in the schematics of the principle setup of the FEL in Fig. 4.4 [47]. This light initially emits spontaneously, but is bounced backwards between a pair of mirrors, just like in a conventional laser until it reaches saturation.

The specific photon energy is set by a resonance between the electrons' velocity and the electromagnetic wave: after each wiggle, the electrons slip back one optical wavelength. This allows a tunability by varying the input electron energy, where in contrast bound-electron-lasers are limited to discrete wavelengths via quantum mechanics.

The set of parameter for the surface treatment in this work are given in Table 4.1.
Table 4.1: Set of parameter for the FEL surface processing: $\mathrm{P}$ is the power, $f_{p, \text { micro }}$ the frequency of the micro-pulse, $\emptyset_{\text {focus }}$ the diameter of the FEL radiation and $\aleph_{s h, i}$ the track displacement of the laser radiation for steel $i$.

\begin{tabular}{ll}
\hline Parameter for the FEL surface processing \\
\hline $\mathrm{P}[\mathrm{W}]$ & 900 \\
$\lambda[\mu \mathrm{m}]$ & 1.64 \\
$f_{p, \text { micro }}[\mathrm{MHz}]$ & 9.36 (in cw-mode) \\
$\emptyset_{\text {focus }}[\mu \mathrm{m}]$ & 600 \\
$\aleph_{\text {sh, AISI310 }[\mathrm{mm}]}$ & 1 \\
$\aleph_{\text {sh,AISI316 }[\mathrm{mm}]}$ & 0.8 \\
\hline
\end{tabular}




\subsection{Experimental Methods}

The following sections describe the experimental analyzing techniques, which are separated into the parts: phase analysis and chemical composition analysis. Each technique will be described briefly. For more detailed explanations, the specific literature will be reported.

\subsubsection{Phase analysis}

\subsubsection{Mössbauer Spectroscopy}

The technique of Mössbauer Spectroscopy is widely used in nuclear solid-state physics, materials science, biophysics and chemistry and bases on the homonymous Mössbauer effect, which was discovered in 1956 by R.L. Mössbauer [48-51]. He showed that nuclear radiation can be emitted and absorbed recoilless, if the atoms are placed in a solid state. Some of the principles, experimental setup, and special magnetic transitions are discussed in this section. Further details are described in the books [52-54].

The Mössbauer Effect A nucleus in an excited state with a mean life time $\tau$ emits $\gamma$-radiation. Its energy distribution can be described by the BreitWigner formula [54]:

$$
I(E)=I_{0} \cdot \frac{(\Gamma / 2)^{2}}{\left(E-E_{0}\right)+(\Gamma / 2)^{2}}
$$

where $E_{0}$ is the transition energy and $\Gamma=\hbar / \tau$ is the resonance width $\left(E_{0}=14.4 \mathrm{keV}, \Gamma=4.7 \mathrm{neV}\right.$ for $\left.{ }^{57} \mathrm{Fe}\right)$. The momentum of the $\gamma$-quantum is $p=\hbar k$. A free atom receive a recoil by an emitted $\gamma$-quantum. Its energy $E_{r}$ can be modeled by $E_{r}=p_{\gamma}^{2} / 2 M=\frac{E_{\gamma}^{2}}{2 M c^{2}}$. The recoil energy is much larger than the natural line width $\Gamma$. If the emitting nucleus is in a solid, the whole matrix takes up the recoil, given that the mass of the matrix is $10^{20}$ times larger than a single, free atom. Thus, the contribution of the recoil energy becomes negligible. This yields in the Mössbauer effect, viz. the resonant nuclear emission and absorption. Nevertheless, the emission (or absorption) may also transfer energy to the lattice, which could results in phononinteractions in the crystal. The probability that the emission takes place without inducing any phonons is named the Mössbauer-Lamb factor, or as analogon to x-ray diffraction, Debye-Waller factor $f_{D}$, which depends on the temperature $T$, the Debye temperature $\theta_{D}$ of the crystal and the energy of the $\gamma$-quantum [55]:

$$
\begin{aligned}
f_{D}(T) & =\exp (x) \\
x & =\gamma\left[1+4\left(\frac{T}{\theta_{D}}\right)^{2} \cdot \int_{0}^{\theta_{D} / T} \frac{y}{\exp (y)-1} \mathrm{~d} y\right]
\end{aligned}
$$

where $\gamma$ is $\frac{-3 \hbar^{2} k^{2}}{3 M k_{B} \theta_{D}}$. The probability of recoil free emission or absorption is 0.76 for ${ }^{57} \mathrm{Fe}$ in $\alpha$-Fe at room temperature. Due to the high resolution involved in this process, the hyperfine interactions can be resolved by a Doppler gamma energy modulation

$$
E(\nu)=E_{0} \cdot\left(1+\frac{\nu}{c}\right)
$$

by moving the source relative to the absorber, or vice versa. The energy resolution of this movement allows the observation of the change of the energy levels resulting from electric or magnetic fields acting at the nucleus. These are the hyperfine interactions: the electric monopole interaction, the electric quadrupole interaction, and the magnetic dipole interaction which will be explained briefly in the next sections.

The isomer shift $\delta$ The electrical monopole interaction is the interaction of the nuclear charge $Z e$ with the electron density, which leads to a change in the energy states in the source $(s)$ and in the absorber $(a)$ in comparison to a point charge. With the energy difference between the absorber and the source, $\delta=E_{a}-E_{s}$, the isomer shift is obtained [56]:

$$
\delta=\frac{2}{5} \pi Z e^{2}\left[\left|\Psi_{a}(0)\right|^{2}-\left|\Psi_{s}(0)\right|^{2}\right]\left[R_{e}^{2}-R_{e}^{2}\right]
$$

where $\Psi$ is the electron wave function, $e\left|\Psi_{s}(0)\right|^{2}$ the electron density and $R_{i}$ the nuclear radius $(i=g \Rightarrow$ $R$ in ground state, $i=e \Rightarrow R$ in excited state).

The quadrupole splitting $\Delta$ An electrical quadrupole moment of the nucleus, $e Q$, interacts with the electric field gradient tensor (EFG) acting at the nucleus, which leads to a splitting of the energy levels. The asymmetry parameter $\eta$ is obtained by diagonalizing the EFG tensor. With the constraints for the electrical potential $V:\left|V_{z z}\right| \geq$ $\left|V_{y y}\right| \geq\left|V_{x x}\right|$ and $V_{x x}+V_{y y}+V_{z z}=0$ the asymmetry parameter can be written as:

$$
\eta=\frac{V_{x x}-V_{y y}}{V_{z z}} .
$$




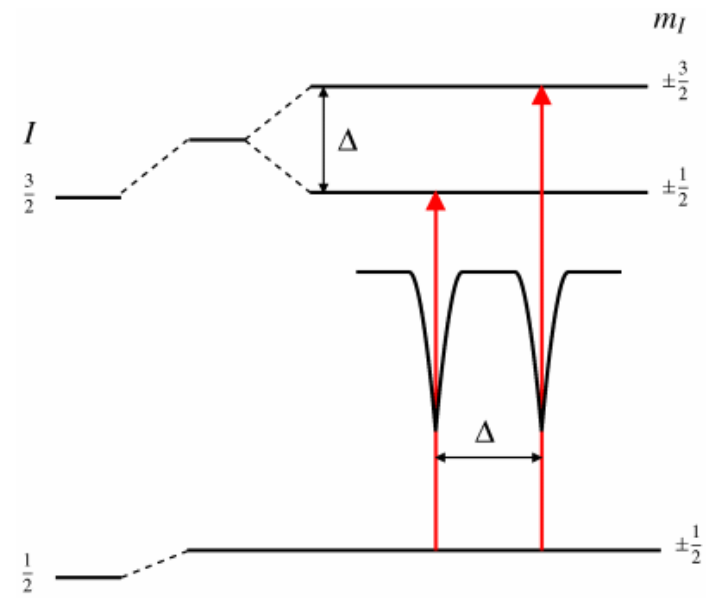

Figure 4.5: Quadrupole splitting for a $3 / 2$ to $1 / 2$ transition. The magnitude of quadrupole splitting $\Delta$ is shown.

In the case of an isotope with a $I=3 / 2$ excited state, such as ${ }^{57} \mathrm{Fe}$ or ${ }^{119} \mathrm{Sn}$, only the excited state is split into two substates $m_{I}= \pm 1 / 2$ and $m_{I}= \pm 3 / 2$, which is shown in Fig. 4.5 By considering the formula for the energy shift given by Wertheim [56], which depends on spin $I$ and magnetic quantum number $m_{I}$, the quadrupole splitting $\Delta$ can be derived as:

$$
\begin{aligned}
\Delta & =E_{q}\left(m_{I}=3 / 2\right)-E_{q}\left(m_{I}=1 / 2\right) \\
& =1 / 2 e Q V_{z z} \sqrt{1+\frac{1}{3} \eta}
\end{aligned}
$$

The magnetic splitting The magnetic moment $\mu$ of the nucleus interacts with a magnetic field $B$ acting at the nucleus (nuclear Zeeman effect). This interaction leads to the splitting of the degenerated states. The state with spin $I$ splits into $2 I+1$ magnetic sub-states with eigenvalues:

$$
E_{m}=-\frac{\mu B m_{I}}{I}=-g_{N} \mu_{N} B m_{I}
$$

where the magnetic quantum number $m_{I}$ can hold the values $m_{I}=I, I-1, \ldots,-(I-1),-I$. The magnetic moment $\mu$ is given by the Bohr magneton $\mu_{N}$ and the Landé factor $g_{N}$ by:

$$
\mu=g_{N} \mu_{N} I \text {. }
$$

A closer look to the selection rule $\left(\Delta_{m}=0 ; \pm 1\right)$ points that only six transitions are possible. This is illustrated in Fig. 4.6.

Mixed hyperfine interaction The previous mentioned formulae are not generally effective, if

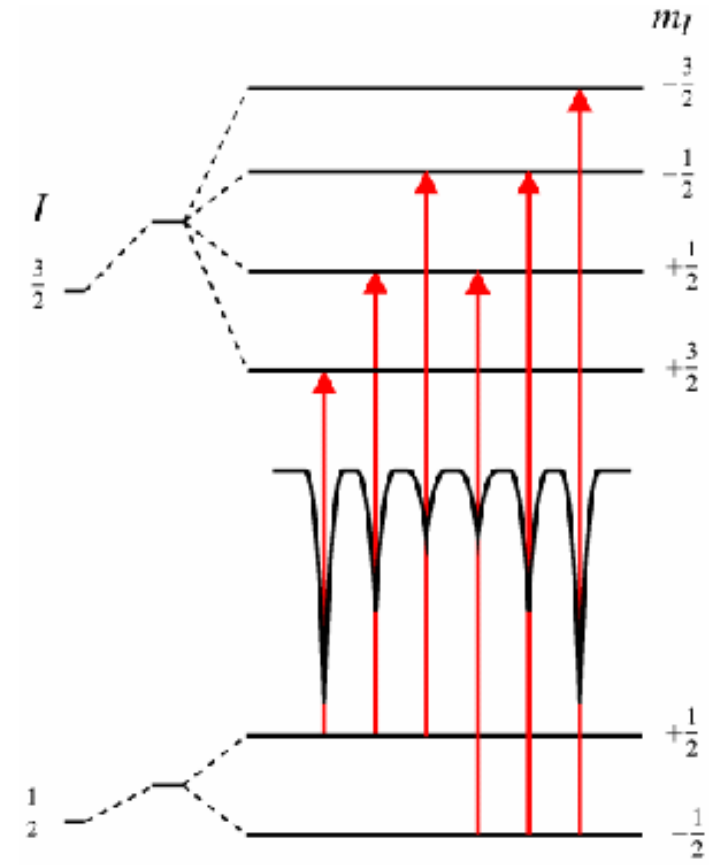

Figure 4.6: Magnetic splitting of the nuclear energy levels.

electric quadrupole and the magnetic dipole interaction appear together. The more general case cannot be described any longer by closed analytic expressions. For Fe-compounds the condition $e Q V_{z z} / 4 \ll$ $\mu_{a} B / I_{a}$ is frequently satisfied, so that the electric quadrupole interaction can be treated as small disturbance of the magnetic dipole interaction. The quantum-mechanical perturbation calculation first order yields in

$$
E_{q}(m, \theta)=\frac{e Q V_{z z}}{4} \frac{3 m^{2}-I(I+1)}{I(2 I-1)} \frac{3 \cos ^{2} \theta-1}{2}
$$

where $\theta$ is the angle between the direction of the magnetic field and the predominant axis of the electric field gradient component $V_{z z}$.

Except the isomer shift, all these hyperfine parameters, are also found for Perturbed Angular Correlation (PAC) [57] and they are comparable with those observed by Mössbauer spectroscopy.

Internal conversion As internal conversion, the process in which an excited nucleus transmits its excessive energy to the orbital electron of the atom, is identified. If the amount of energy given to the orbital electron exceeds its binding energy, it leaves the atomic union. Therefore, x-rays are emitted as the atom attempts to restore its neutrality. Internal conversion usually competes with gamma radiation as an energy-shedding mechanism.

Internal conversion is schematically illustrated in 


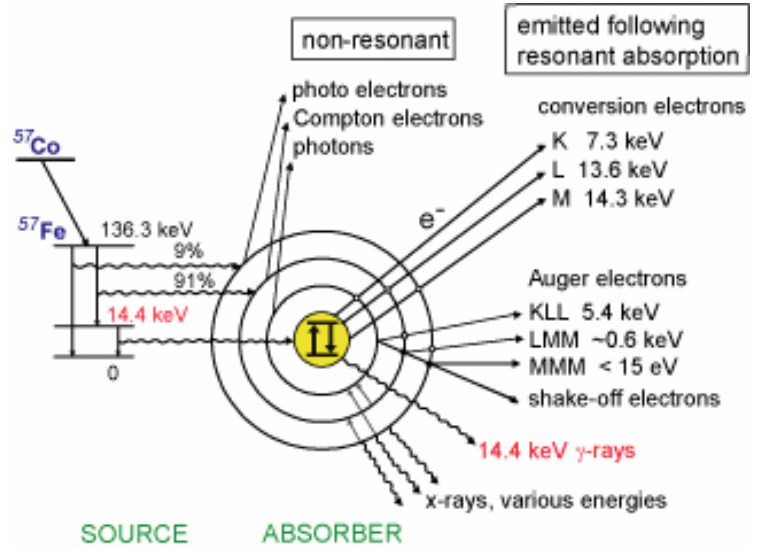

Figure 4.7: Process scheme of the internal conversion for ${ }^{57} \mathrm{Fe}$.

Fig. 4.7 [58].

From this scheme, the different Mössbauer measuring proceedings can be derived: Transmisson Mössbauer Spectroscopy (TMS), Conversion Electron Mössbauer Spectroscopy (CEMS) with an information depth of $150 \mathrm{~nm}$ [59], Conversion X-ray Mössbauer Spectroscopy (CXMS) with an information dept of 30-50 $\mu \mathrm{m}$ [59] and Auger Electron Mössbauer Spectroscopy (AEMS), which is very surface sensitive [60]. In this work, CEMS measurements were performed [61-63]. In Fig. 4.8 the schematics of the detector is shown and is described in [59]. A mixture of helium and methane is flow-

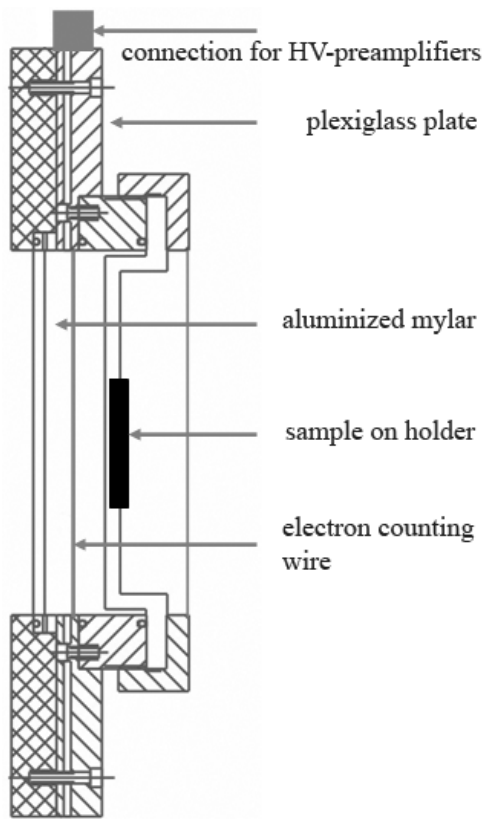

Figure 4.8: Mechanical drawing of the CEMS detector. ing through the counting tube $(94 \mathrm{Vol} . \%$ He and 6 Vol. $\% \mathrm{CH}_{4}$ ) to prevent a contamination of the test gas.

\subsubsection{Extended X-ray Absorbtion Fine- Structure}

$\mathrm{X}$-rays are electromagnetic waves with energies ranging from $\sim 500 \mathrm{eV}$ to $500 \mathrm{keV}$. At this energy regime, a X-ray beam is absorbed by the photoelectric effect, if the beam traverse the solid. Absorbtion is primarily characterized by the absorbtion coefficient $\mu$, which gives the probability that X-rays will be absorbed according to Beer's Law:

$$
I=I_{0} e^{-\mu t}
$$

where $I_{0}$ is the X-ray intensity incident on a sample, $t$ the sample thickness, $I$ the intensity transmitted through the sample and where $\mu$ is given by $\mu \sim \frac{\rho Z^{4}}{A E^{3}} . \quad \mu$ is a smooth function of energy, which depends on the sample density $\rho$, the atomic number $Z$, atomic mass $A$ and the X-ray energy $E$. As well as for all electromagnetic waves, the intensity of X-rays is proportional to the number of photons. Due to the $Z^{4}$ dependence, the absorption coefficient for usual chemical compounds are very different, so that good contrast between different materials can be achieved for nearly any sample thickness and concentrations by adjusting the X-ray energy.

$\mathrm{X}$-ray absorbtion fine structure (XAFS) is generally thought of in two distinct portions: the nearedge spectra (XANES) - typically within $30 \mathrm{eV}$ of the main absorption edge, and the extended fine-structure (EXAFS) which contains information about the local atomic neighborhood. The basic physical description of these two regimes is the same, but important approximations and limits allow to interpret the extended spectra in a more quantitative way than those for the near edgespectra.

EXAFS refers to the oscillations above the absorption edge [64]. Its fine structure function $\chi(E)$ is defined as:

$$
\chi(E)=\frac{\mu(E)-\mu_{0}(E)}{\Delta \mu_{0}(E)}
$$

where $\mu(E)$ is the measured absorption coefficient, $\mu_{0}(E)$ is a smooth background function representing the absorption of an isolated atom, and $\Delta \mu_{0}$ is the measured jump in the absorption $\mu(E)$ at the threshold energy $E_{0}$, which can be seen in Fig. 4.9.

As mentioned above, EXAFS is understood in terms of photo-electron created wave behavior in the absorbtion process. Therefore, it is common to 


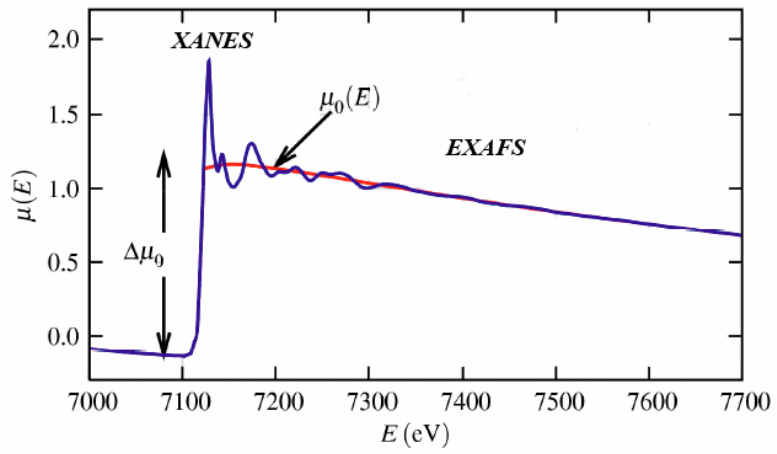

Figure 4.9: Typical XAFS spectrum for Fe. The XANES and EXAFS regions are identified. $\mu(E)$ is shown with the smooth background function $\mu_{0}(E)$ and the edge step $\Delta \mu_{0}\left(E_{0}\right)$.

convolute the X-ray energy to the wave number $k$, which is defined as:

$$
k=\sqrt{\frac{2 m_{e}\left(E-E_{0}\right)}{\hbar^{2}}}
$$

where $E_{0}$ is the absorption edge energy and $m_{e}$ the electron mass. Thus, the fine structure function has the quantity $\chi(k)$ and decays quickly with $k$. As a result, $\chi(k)$ typically is often scaled by $k, k^{2}$ or $k^{3}$. In this work, the spectra are multiplied with $k^{2}$.

The oscillations correspond to quantuminterferences caused by the different near-neighbor coordination shells and can be described by the equation:

$$
\chi(k)=\sum_{j} \frac{N_{j} f_{j}(k) e^{-2 k^{2} \sigma_{j}^{2}}}{k R_{j}^{2}} \sin \left[2 k R_{j}+\delta_{j}(k)\right]
$$

where $f(k)$ and $\delta(k)$ are the scattering properties of the next nearest-neighborhood, $N$ the number of neighboring atoms, $R$ the distance between two atoms, and $\sigma^{2}$ is the disorder in the neighbor distance. By getting the scattering amplitude $f(k)$ and phase-shift $\delta(k)$ from the experiments, a determination of $N, R$ and $\sigma^{2}$ is possible. As a result of the $\mu$ dependency of $\chi(k)$, the fine structure function also depends on $\mathrm{Z}$ of the next-nearest atoms. Thus, EXAFS is also sensitive to atomic species of the neighborhood.

EXAFS experiments in this work were carried out at the European Synchrotron Radiation Facility (ESRF (Grenoble), France) on the beamline BM29. The storage ring was operating at $6 \mathrm{GeV}$ with an average current of $200 \mathrm{~mA}$. The data were collected at room temperature in the $7000-7700 \mathrm{eV}$ (Fe Kedge: $\left.E_{0}=7112.0 \mathrm{eV}\right)$ and in the $8200-8900 \mathrm{eV}$
(Ni K-edge: $E_{0}=8332.0 \mathrm{eV}$ ) energy range with 1 $\mathrm{eV}$ step in total electron yield (TEY) mode. The data analysis was performed with the HORAE software package [65-67]. For the sake of completeness, the main parameters of the electron beam in the storage ring are summarized in Table 4.2.

Table 4.2: Parameters of the electron beam in the ESRF storage ring .

\begin{tabular}{l|c|l}
\hline Energy & $\mathrm{GeV}$ & 6.03 \\
Max. Current & $\mathrm{mA}$ & 200 \\
Horizontal Emittance & $\mathrm{nm}$ & 4 \\
Vertical Emittance (*min. achieved) & $\mathrm{nm}$ & $0.025\left(0.010^{*}\right)$ \\
Coupling (*min. achieved) & $\%$ & $0.6\left(0.25^{*}\right)$ \\
Revolution frequency & $\mathrm{kHz}$ & 355 \\
Number of bunches & & 1 to 992 \\
Time between bunches & $\mathrm{ns}$ & 2.816 to 2.82 \\
\hline
\end{tabular}

\subsubsection{X-ray diffraction}

$\mathrm{X}$-ray diffraction (XRD) is a general characteristic of all waves and is referred to the rearrangement of the behavior of waves by its interaction with an object. It is used for structural analysis by revealing the phase difference $\Delta_{i}$ of the characteristic interference patterns of scattered X-rays photons at each lattice plain. The physical values derived from the patterns, such as position, shape and intensity provide detailed information on the structural properties of the examined specimen on atomic scales. Fig. 4.10 [68] indicates the condition for constructive interference, where the phase shift adds to a multiple of the incident wavelength .

By considering the trigonometric conditions, the

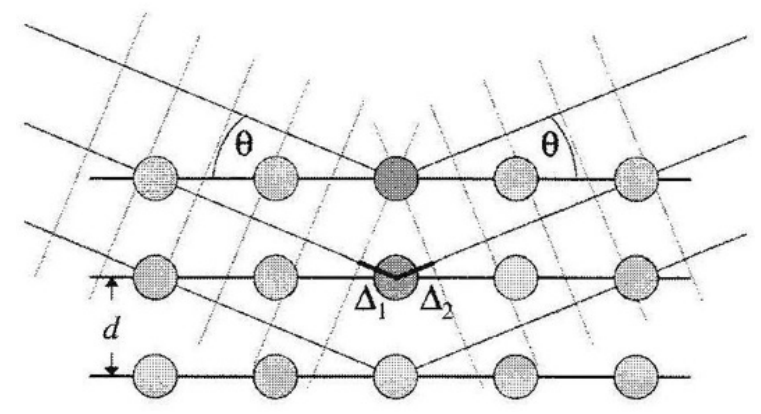

Figure 4.10: Visualization of the so-called Bragg equation. Maximum scattered intensity is only obtained when the phase shifts add to a multiple of the incident wavelength $\lambda$.

sum of phase difference lead to the Bragg equation $[69,70]$ :

$n \lambda=\Delta_{1}+\Delta_{2}=2 d \cos \left(90^{\circ}-\theta\right)=2 d \sin \theta$ 
where $d$ is the distance of two parallel plains and $\theta$ the angle with respect to the incident X-ray beam of wavelength $\lambda$.

Another approach to explain diffraction is given by Laue [71] as seen in Fig. 4.11. The path difference of

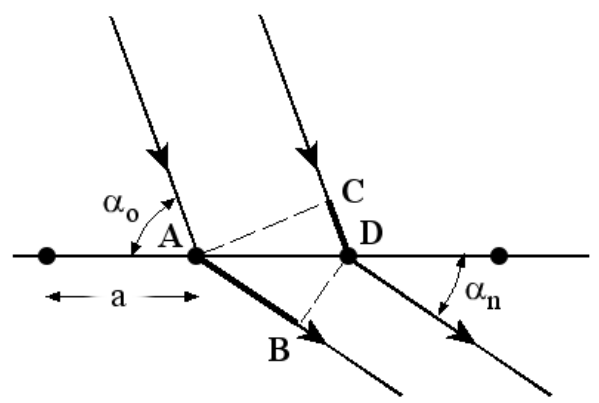

Figure 4.11: Laue diffraction geometry.

rays scattering at points $\mathrm{A}$ and $\mathrm{D}$ is just $\mathrm{AB}-\mathrm{CD}$. If the incoming rays are in phase, the path difference must be some integral multiple of the wavelength for constructive interference to occur. This lead to the first Laue equation:

$$
(A B-C D)=a\left(\cos \alpha_{n}-\cos a_{0}\right)=n_{x} \lambda
$$

This result is valid for any scattered ray that makes an angle an with the unit cell axis. Thus the Laue condition is consistent with a cone of scattered rays centered about the a axis. This equation can be restated in vector terms. The repeat distance a, becomes a unit cell vector a. Call a unit vector parallel to the incoming rays, $k_{0}$, and one parallel to the scattered rays, $S$. There are then some simple vector dot products as seen in Fig. 4.12:

$$
\begin{aligned}
a \cdot S & =a \cdot \cos \alpha_{n} \\
a \cdot S_{0} & =a \cdot \cos \alpha_{0} \\
a\left(\cos \alpha_{n}-\cos \alpha_{0}\right) & =a \cdot\left(S-S_{0}\right)=n_{x} \lambda
\end{aligned}
$$

The $\theta / 2 \theta$ scan An often-used instrument for measuring Bragg reflections of thin films is the $\theta / 2 \theta$ diffractometer. In order to derive an information on the microstructure of an given sample, the measurement is set up to investigate position, shape and intensities of the reflections. The intensity function can be obtained by the square of the absolute value of the structure factor $F_{h k l}$ multiplicated with the polarization factor $p$ :

$$
\begin{aligned}
I_{h k l}(Q) & =\left|F_{h k l}\right|^{2} p \\
& =\prod_{i=1}^{3} \frac{\sin ^{2}\left(N_{i} a Q c_{i} / 2\right)}{\sin ^{2}\left(a Q c_{i} / 2\right)}
\end{aligned}
$$

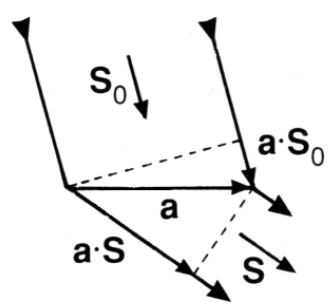

Figure 4.12: Vectorial display of the Laue diffraction geometry.

where $Q=S-S_{0}, N_{i}$ is the normal vector and $c_{i}$ vectors in reciprocal space.

The intensity variation that is associated with the reflection is given by the above mentioned equation, while the scattered intensity depends on the distance from the sample to the detector system. Therefor, the instrument should be configured in such a way, that the scan can measure the space around the sample by keeping the sample-detector distance constant.this measure ensures that any intensity variation is due to the intensity function and is not caused by a dependency of the sampledetector distance. the detector should accordingly move on a semicircle in 2-D, on a hemisphere in 3$\mathrm{D}$, respectively, with constant radius with the sample in the center of it. The geometry is shown in Fig. 4.13 .

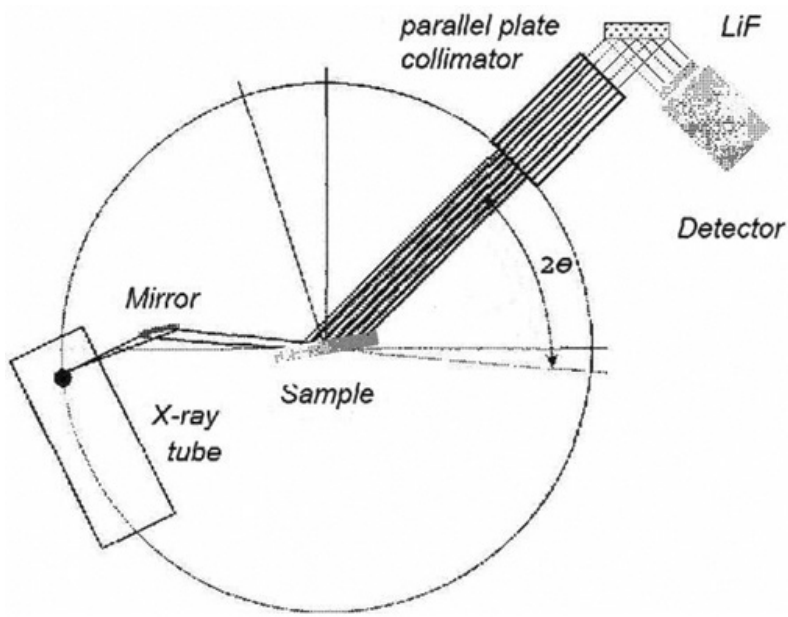

Figure 4.13: The $\theta / 2 \theta$ geometry. The x-ray tube is operated in the line focus mode; the plane of the figure is the scattering plane.

Gracing Incidence X-ray diffraction The gracing incidence measuring configuration is shown 
in Fig. 4.14.

The angle between the incoming beam and the

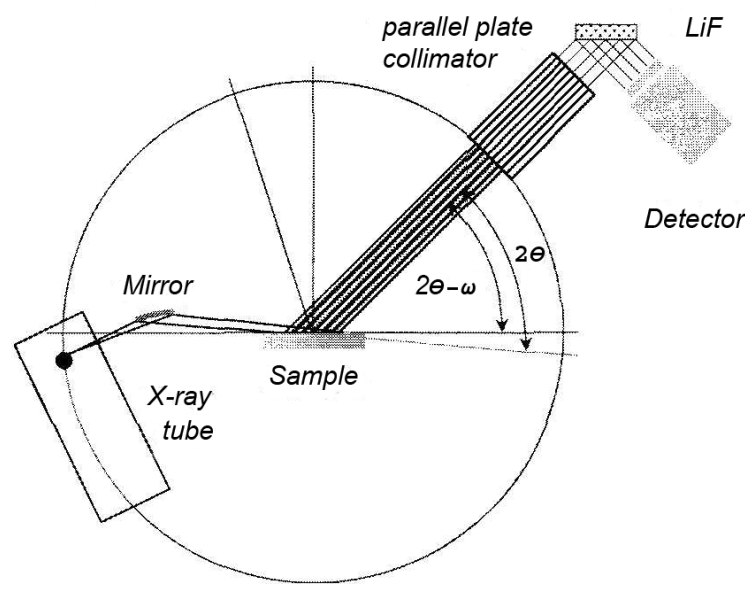

Figure 4.14: The GIXRD geometry. The x-ray tube is operated in the line focus mode; the plane of the figure is the scattering plane.

sample surface is very small and amounts to only a few degrees. This angle is different from half the scattering angle and will be denoted as $\omega$. The Xrays travel a maximum path of $l=\sin \omega$ within the layer, which might be a high multiple of the thickness $t$ when the entrance angle is chosen very flat. For the investigation of a sample with attenuation coefficient $\mu$ a first criterion for $\omega$ should be $l=1 / \mu$, so that for the incidence angle the condition $\omega=\arcsin (\mu t)$.

To get an insight to the information depth of the penetrating beam, the GIXRD absorbtion factor $A_{\omega}=\left(1-\exp \left(-\mu t k_{\omega}\right)\right.$ and a machine dependant configuration factor $k_{\omega}$ has to be taken into account. By considering, that average information depth $\overline{\tau_{\omega}}$ is the weighted sum of all beams that differ by the amount of damping they undergo by being diffracted in various depths, $\overline{\tau_{\omega}}$ is obtained as [68]:

$$
\overline{\tau_{\omega}}=\frac{1}{\mu k_{\omega}}+\frac{t}{1-\exp \left(\mu t k_{\omega}\right)} .
$$

In this work, $\omega$ was fixed to $2^{\circ}$. With the formula for $\overline{\tau_{\omega}}$ we get an information depth of $\overline{\tau_{\omega}} \approx 350 \mathrm{~nm}$.

The Bruker AXS D8 Advance diffractometer In this work, crystallographic analyses were performed using a Bruker AXS D8 diffractometer equipped with a $\mathrm{Cu}-\mathrm{K} \alpha$ source $(\lambda=1.54 \AA)$, a Goebel mirror, a grazing incidence attachment mounted on an eulerian gradle, a soller-collimator system, a secondary monochromator $(\mathrm{LiF})$ and a YAP-detector.

\subsubsection{Transmission Electron Microscopy}

The transmission electron microscopy is used in physics, chemistry, biology, in the medicine and in the engineering sciences for the clearing-up of most different questions. Examples are the exploration of the atomic structure of inorganic or organic materials, the illustration of biological objects with high lateral resolution or the visualization of the structure and the chemical composition of very finecrystalline materials. The small wavelength of the electrons permits thereby a lateral resolution up to atomic dimensions.

Based on the de Broglie relationship, the wavelength $\lambda$ of the electron is related to its momentum $p$ through Planck's constant $h$ :

$$
\lambda=\frac{h}{p}
$$

For an electron which experiences the accelerating voltage $U_{A}$, the equation from the relativistic energy theorem can be derived:

$$
\begin{aligned}
E & =\sqrt{p^{2} c^{2}+m_{0}^{2} c^{4}}=m_{0} c^{2}+e U_{A} \\
\Rightarrow p & =\sqrt{2 m_{0} e U_{A}\left(1+\frac{e U_{A}}{2 m_{0} c^{2}}\right)}
\end{aligned}
$$

If one inserts the impulse into the previously mentioned relationship, the wavelength $\lambda$ of an electron which experienced the accelerating voltage $U_{A}$, can be written as:

$$
\begin{aligned}
\lambda & =\frac{h}{\sqrt{2 m_{0} e U_{A}\left(1+\frac{e U_{A}}{2 m_{0} c^{2}}\right)}} \\
{[n m] } & \approx \sqrt{\frac{1.5}{U_{A}[V]} .}
\end{aligned}
$$

Fig. 4.15 shows the wavelength $\lambda$ of the electrons as a function of the accelerating voltage $U_{A}$.

At accelerating voltages of $200 \mathrm{kV}$ and $300 \mathrm{kV}$, the wavelengths are of the magnitude $2.74(2) \mathrm{pm}$ and $2.25(3) \mathrm{pm}$, respectively. Since the increase of the accelerating voltage has only a small contribution to the resolution, accelerating voltages of $200 \mathrm{kV}$ are used in conventional TEMs. Theoretically, resolutions in the sub-nanometer scale can be achieved. Practically, only point resolutions of $0.12 \mathrm{~nm}$ and line resolutions of $0.10 \mathrm{~nm}$ were achieved for $1 \mathrm{MeV}$ TEMs, which are called Ultra High Voltage Electron Microscopes (UHVEM) [72]. The limitations in resolution are given by spherical and chromatic aberration. World-wide, some working groups are resolving these limitations by electron-optical elements like the corrector for the spherical aberration 


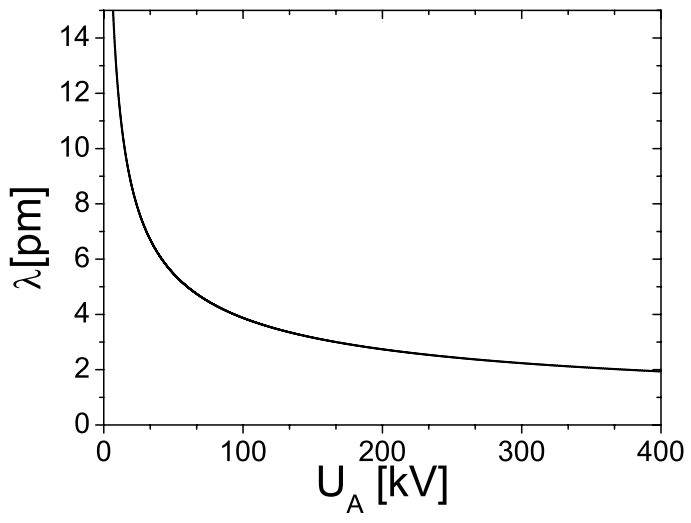

Figure 4.15: The wavelength $\lambda$ of the electrons as a function of the accelerating voltage.

("Cs-corrector") or by holographic recording methods like off-axis electron holography and focus-series reconstruction.

The principle setup of a TEM Similar to a light microscope, a TEM can be divided into three components: 1) an illumination system, 2) the objective lens, and 3) the imaging system. The role of the illumination systems (condenser) is to transfer the electrons from the source to the specimen, which is realized in two different modes: a parallel or a convergent beam mode. The first one is used for TEM imaging and selected area diffraction (SAD), while the second one is used for imaging in a Scanning Transmission Electron Microscope (STEM), which are used for microanalysis and microdiffraction. As indicated above, the objective lens is the most important part of the TEM. It produces the first image of the sample in its image plane and the diffraction pattern in its back focal plane and its resolution depends primarily and sensitively on the aberration of the objective lens and secondary on the high voltage. Image or diffraction pattern can be selected with help of the intermediate lens, which images either the back focal plane or the image plane of the objective lens as seen in Fig. 4.16 [73]. Further magnification on the screen is performed by subsequent projector lenses.

A bright-field image (BF) is formed when only the direct beam is used for image formation. The objective aperture blocks all other beams to pass to the imaging system. A dark-field image (DF) is formed when the aperture is positioned to pass only some scattered electrons. The off-axis electrons are less accurately bent due to spherical aberrations of the objective lens which leads to a reduced image quality. To avoid this, the incident beam has to be tilted above the objective lens to keep the beam,

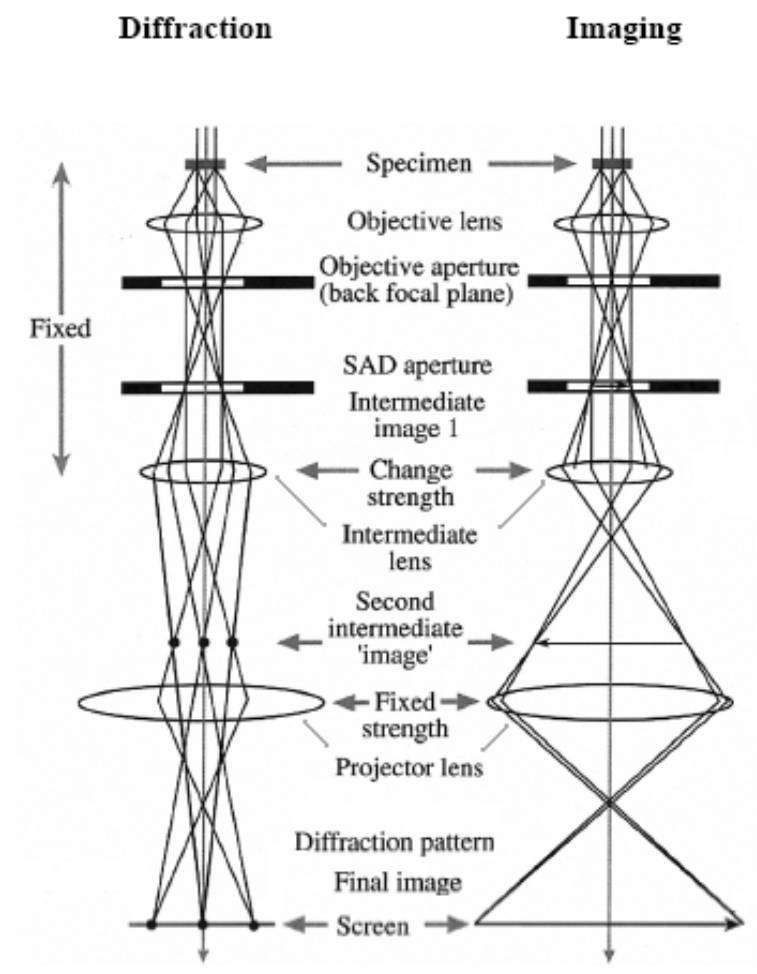

Figure 4.16: The wavelength $\lambda$ of the electrons as a function of the accelerating voltage.

which is used for imaging, close and parallel to the optic axis.

The Philips CM 200-UT microscope In this work, transmission electron microscopy was carried out employing a Philips CM 200-UT microscope (200 kV acceleration voltage) and which is equipped with a Noran Energy Dispersive X-ray detector for STEM. The achieved point resolution was $0.187 \mathrm{~nm}$, the resolving capacity $0.11 \mathrm{~nm}$. 


\subsubsection{Chemical composition analysis}

\subsubsection{Rutherford Backscattering Spec- trometry}

Rutherford backscattering spectroscopy (RBS) is one of the most powerful techniques for measuring elemental depth profiles. It allows quantitative and nondestructive analysis with a reasonable depth resolution. In conventional RBS, He ions with energies in the range $1-4 \mathrm{MeV}$, are used as probes. Almost all ions penetrate deep inside the sample up to ca. $10 \mu \mathrm{m}$ until they entirely lose their kinetic energy. During the penetration some ions collide with the target atoms and are subject to elastic Coulomb scattering (Rutherford scattering) between the projectile and the target nuclei and may be backscattered from the sample. The energy of the ion backscattered from the target atom depends on the target atom mass. This allows compositional analysis of a surface region of several micrometers by measuring the energy spectrum of the backscattered ions.

Kinematics of collisions When an ion is scattered from a target atom at an angle $\theta$, the ratio of the scattered-ion energy $E$ to the incident energy $E_{0}$ can be calculated using the laws of conservation of energy and momentum [74],

$$
k=\frac{E}{E_{0}}=\frac{\sqrt{M_{2}^{2}-M_{1}^{2} \sin ^{2} \theta}+M_{1} \cos \theta}{M_{1}+M_{2}}
$$

where $M_{1}$ and $M_{2}$ are masses of the incident ion and the target atom, respectively. The binding energy of the target atom and its thermal vibration are neglected in this derivation. These effects are negligibly small in collisions between ions with megaelectron volt energy and target atoms. It is clear that the energy ratio $\mathrm{K}$, called the kinematic factor, is determined by the mass ratio $M_{2} / M_{1}$ and the scattering angle $\theta$. The basic idea of RBS relies on this equation.

The Rutherford Cross-section The scattering cross-section $\sigma$ is an effective area associated with the colliding particles. When projectile ions collide on a thin target film of thickness $t$, the scattering yield is given by [74]

$$
Y_{t o t}=\frac{N t \sigma Q}{\cos \alpha}
$$

where $N$ is the volume density of the target atom, $Q$ the number of incident ions, and $\alpha$ is the incident angle of the beam in respect to the normal vector of the target.

In RBS, the ions scattered at a particular scattering angle $\theta$ are measured by a detector with a finite acceptance solid angle $\Delta \Omega$. The number of these ions can be calculated with an angular differential cross-section [74]

$$
Y(\theta)=N t \frac{d \sigma}{d \Omega} \frac{\Delta \sigma Q}{\cos \alpha} .
$$

The differential cross-section for Coulomb scattering (Rutherford cross-section) is given by the Rutherford formula [74]:

$$
\begin{aligned}
\left(\frac{d \sigma}{d \Omega}\right)_{R} & =\left(\frac{Z_{1} Z_{2} e^{2}}{4 E}\right)^{2} \\
& \times \frac{4 \cdot\left[\sqrt{M_{2}^{2}-M_{1}^{2} \sin ^{2} \theta}+M_{1} \cos \theta\right]^{2}}{M_{2} \sin ^{4} \theta \sqrt{M_{2}^{2}-M_{1}^{2} \sin ^{2} \theta}}
\end{aligned}
$$

where $Z_{1}, Z_{2}$ are atomic numbers of incident ion and target atom, respectively and $E$ is the incident energy. This simple formula allows to extract quantitative data about abundances of elements from RBS spectra. The cross-section is proportional to $Z_{2}^{2}$, indicating high sensitivity for heavy elements in RBS.

The IONAS Accelerator Facility RBS was performed at the $530 \mathrm{kV}$ IONAS accelerator facility in Göttingen, which is described in $[75,76]$ and which is shown in Fig. 4.17. In Table 4.3, the parameters for all RBS measurements are given.

Table 4.3: Parameters of all RBS measurements at the IONAS facility in Göttingen .

\begin{tabular}{ll}
\hline Probe ion & $\mathrm{He}^{2+}$ \\
Ion energy & $0.9 \mathrm{MeV}$ \\
Beam current & $10-15 \mu \mathrm{A}$ \\
Beam spot size (focused) & $2 \mathrm{~mm}^{2}$ \\
Detector & silicon surface barrier (SSB) \\
Detector resolution & $12.5 \mathrm{keV}$ (FWHM) \\
Scattering angle $\theta$ & $165^{\circ}$ \\
Depth resolution & $\sim 10 \mathrm{~nm}$ \\
Element range & $>4$ \\
Sensitivity & ca. $100 \mathrm{ppm}$ for heavy elements \\
& in light hosts \\
\hline
\end{tabular}

\subsubsection{Resonant Nuclear Reaction Analy- sis}

As well as RBS, Resonant Nuclear Reaction Analysis (RNRA) is a powerful tool for depth profiling. In contrast to RBS, where all elements of an examined sample were instantaneously profiled via the scattering process, RNRA has a high sensitivity to one 


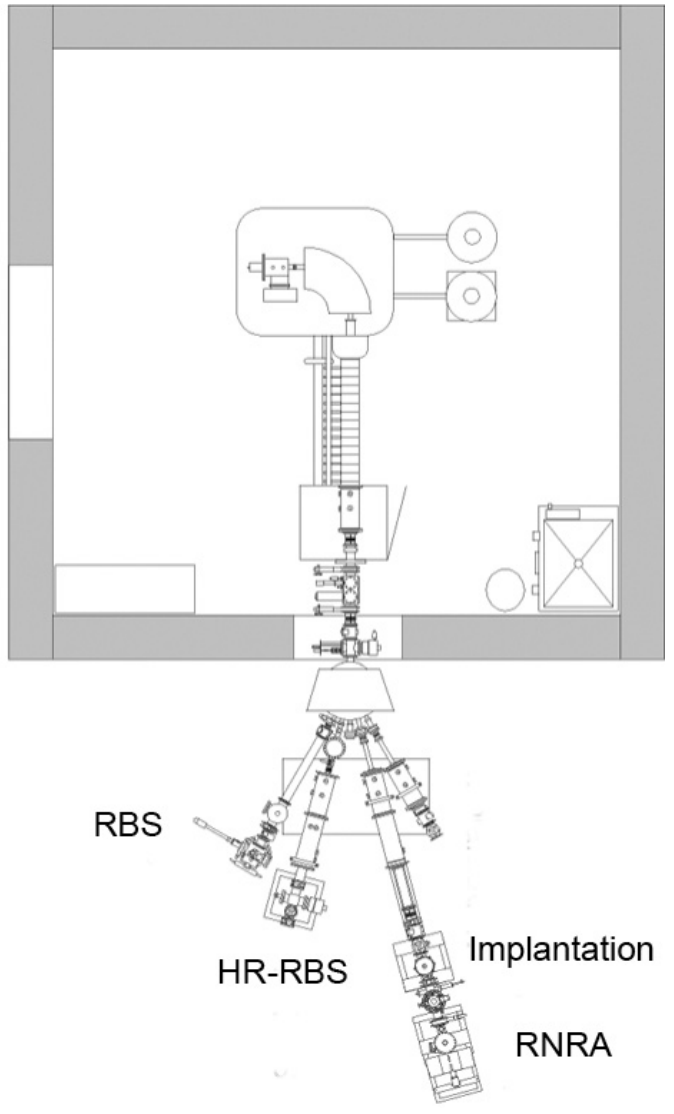

Figure 4.17: Schematics of the IONAS accelerator.

specific element due to a nuclear reaction, in this case the ${ }^{15} \mathrm{~N}(p, \alpha \gamma){ }^{12} \mathrm{C}$ reaction [77]. the theory of RNRA is well discussed in $[78,79]$ and is ideal for nitrogen depth profiling [80-82]. The schematics of RNRA is shown in Fig. 4.18 [83].

The Yield of the $4.43 \mathrm{MeV}$ emitted $\gamma$-ray can be

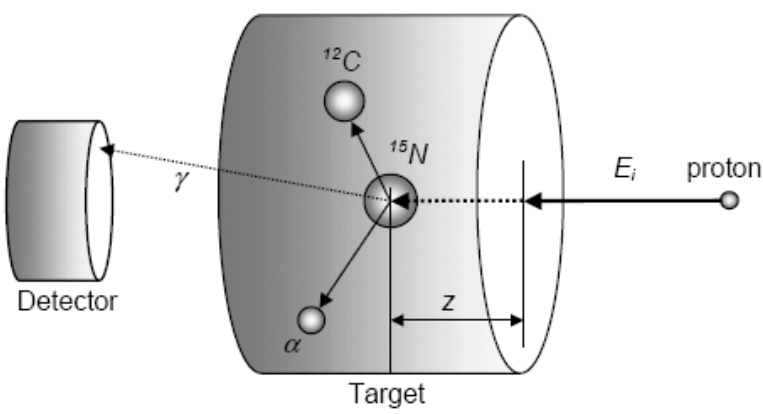

Figure 4.18: Schematics of the RNRA process. described by:

$$
\begin{aligned}
Y\left(E_{b}\right) & =\epsilon_{d e t} \Omega N_{p} \int_{0}^{\infty} \int_{0}^{\infty} \int_{0}^{\infty} C_{N}(z) g\left(E_{b}, E\right) f\left(E, E^{\prime}, z\right) \\
& \cdot \sigma\left(E^{\prime}\right) \mathrm{d} E^{\prime} \mathrm{d} E \mathrm{~d} z
\end{aligned}
$$

where $\epsilon_{d e t}$ is the efficiency of the detector, $\Omega$ the detector solid angle, $N_{p}$ the number of incident protons and $C_{N}(z)$ the concentration profile of nitrogen atoms. The most important factors in the formula are $\sigma\left(E^{\prime}\right)$ and $f\left(E, E^{\prime}, z\right)$. the first one describes the resonance cross section at an proton energy $E^{\prime}$, whereas the second one corresponds to the presence of a proton with initial energy $E$, after penetrating a depth of $\mathrm{z}$ by maintaining the energy $E^{\prime}$.

The nitrogen depth profiles and the proton incidence energy define the observed $\gamma$-yield. Vice versa, the $C_{N}(z)$ of a binary compound $A_{M} B_{n}$ can be derived by a Fourier retransformation:

$$
C_{N}=\frac{C_{s t} Y_{\epsilon B}}{Y_{s t \epsilon B}+C_{s t} Y\left(\epsilon_{B}-\epsilon_{A}\right)}
$$

where $\epsilon_{A}$ and $\epsilon_{A}$ describe the stopping power of element $\mathrm{A}$ and $\mathrm{B}, \epsilon_{s t}$ the stopping power of the calibration sample (here a magnetron sputtered TiN, $400 \mathrm{~nm}$ in thickness), $C_{s t}$ the nitrogen concentration of the calibration sample and $Y, Y_{s t}$ the $\gamma$-yield of $A_{M} B_{n}$ and of the calibration sample, respectively. the resulting stopping power of $A_{M} B_{n}$ is then derived by the Bragg rule [84].

By assuming a constant stopping power in an infinitesimal small depth-element, the analyzing depth can be derived by

$$
z\left(E_{i}\right)=z\left(E_{i-1}\right)+\frac{2\left(E_{i}-E_{i-1}\right.}{\epsilon\left(E_{i}\right)+\epsilon\left(E_{i-1}\right)} .
$$

The observed spectra were analyzed by the WinRNRA fitting tool [85].

\subsubsection{Other Methods}

Perturbed angular correlation (PAC) spectroscopy was performed using ${ }^{111} \mathrm{Cd}$ atoms as PAC probe, which are populated by the $2.8 \mathrm{~d}$ EC decay of ${ }^{111}$ In. A recent description of the method as applied in Göttingen can be found in [86]. Using a four detector set-up, 12 coincidence time spectra were recorded. Combining these spectra, the perturbation function $R(t)$ is derived, which is proportional to the angular correlation coefficient $A_{22}^{\text {eff }}$ and the perturbation factor $G_{22}^{i}(t)$. The spectra were fitted by:

$$
F(t)=A_{22}^{i} \cdot \sum_{i} f_{i} \cdot G_{22}^{i}(t)
$$


with

$$
\begin{aligned}
G_{22}^{i}(t) & =\sum_{n=0}^{3} s_{2 n}\left(\eta^{i}\right) \cdot \cos \left(g_{n}\left(\eta^{i}\right) \cdot \nu_{Q}^{i} t\right) \\
& \cdot \exp \left(-g_{n}\left(\eta^{i}\right) \cdot \delta^{i} t\right)
\end{aligned}
$$

The fraction $f^{i}$ of probe atoms is exposed to an electric field gradient (EFG) characterized by the quadrupole interaction frequency $\nu_{Q}^{i}$ and the asymmetry parameter $\eta^{i}$. Further information on the principles of PAC can be obtained in $[57,87]$. Magneto-optical Kerr effect (MOKE) was used to investigate the ferromagnetic behavior of the samples. The MOKE measurements were conducted in longitudinal geometry at room temperature, using a polarization-compensator-sample-analyzer (PCSA) ellipsometer and a maximum external magnetic field of $0.15 \mathrm{~T}[88,89]$. The samples were mounted onto a rotationally motorized holder, so that magnetic anisotropy could also be analyzed by rotating the sample with respect to the direction of the magnetic field [90].

Atomic force microscopy (AFM) was performed on a Nanoscope III MultiMode AFM (Digital Instruments) whose maximum scan range is $80 \mu \mathrm{m}$ $\times 80 \mu \mathrm{m}$. All samples were measured in tappingmode.

Scanning tunneling microscopy (STM) was performed on a Nanosurf Easyscan II STM (nanoScience Instruments) whose maximum lateral scan range is 1 micron.

Raman spectroscopy was carried out on a selfassembled device. An argon laser with a wavelength of $514.5 \mathrm{~nm}$ was used, together with a Dilor XY triple monochromator. The optics track was assigned to the so-called macro-Raman optics in which the laser beam is aligned in grazing incident mode. The emitted Raman radiation was mapped by a CCD camera.

Corrosion resistance tests used a combination of two galvanic cells. The sputtered films were used as working electrodes, a platinum and kalomel electrode as counter- and as reference-electrode, respectively. 1-molar $\mathrm{Na}_{2} \mathrm{SO}_{4}$ was used as electrolyte and the voltage was varied from -2 to $+2 \mathrm{~V}$ by means of a potentiostat [91].

Nanoindentation measurements were performed on a Fischerscope HV100 with a Vickers diamond. The maximum indention force was set from 2 to $5 \mathrm{mN}$. Five positions were measured for each sample and the mean values are reported [92]. 


\section{Chapter 5}

\section{Targets and starting materials}

In this section, the physical properties of the used targets are specified.

The original ARMCO-Iron (purity $99.96 \%$ ) and

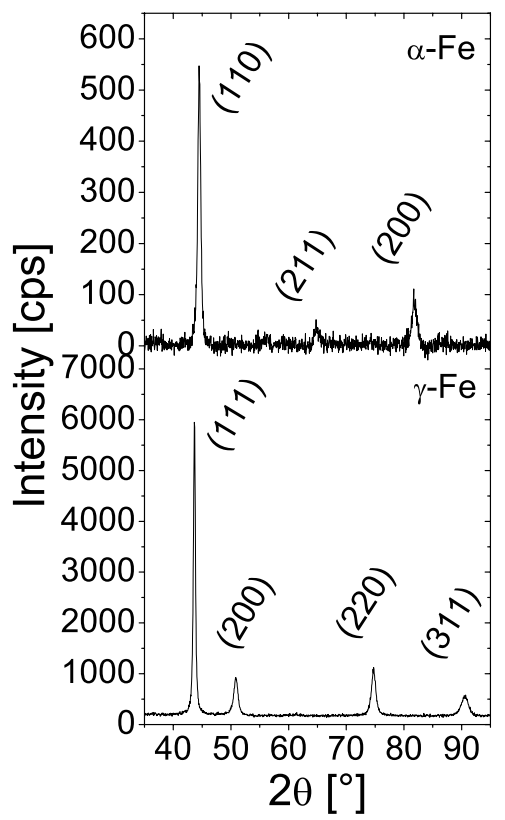

Figure 5.1: top: GIXRD ( $2^{\circ}$ incidence angle) pattern of the ARMCO-Iron sputter target; bottom: $\theta-2 \theta$ pattern of the AISI 316 sputter target.

AISI 316 targets have a body-centered cubic (bcc, $\alpha$ ) and face-centered cubic(fcc, $\gamma$ ) structure, respectively, in contrast to inert magnetron-sputtered thin AISI 316 films, which exhibit only an $\alpha$-structure structure when deposited below $678 \mathrm{~K}$ [93]. This is readily seen in Fig. 5.1, where the XRD patterns of ARMCO-Iron and AISI 316 are shown.

The bcc structure of the original ARMCO-Fe target and the fcc structure of the original AISI 316 target are extracted in Fig. 5.1a) and b). The lattice constants obtained are $a=0.2881(1) \mathrm{nm}$ for the bcc ARMCO target and $a=0.3592(1) \mathrm{nm}$ for the fcc stainless steel target.

This behavior is resembled in the Mössbauer spec- tra of the targets as shown in Fig. 5.2.

The Mössbauer spectrum of the original ARMCO

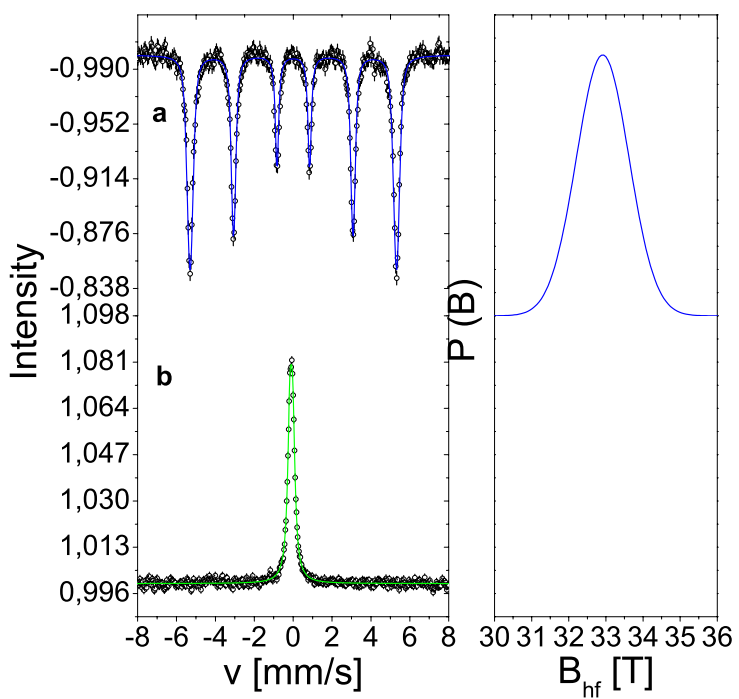

Figure 5.2: (a) Transmission Mössbauer spectrum of the original ARMCO-Iron sputter target; (b) CEM spectrum of the original AISI 316 sputter target. The corresponding distributions of the hyperfine fiels $p(B)$ are given on the right.

target shows, as mentioned in the theory section, a split Zeeman sextet (Fig. 5.2 a) due to the magnetic splitting of the nuclear energy levels. From the fitting, a mean hyperfine field of $\langle B\rangle=32.9 \mathrm{~T}$ with a width of $\sigma=0.7 \mathrm{~T}$ is obtained from the $p(B)$ distribution. In contrast, the original AISI 316 target in Fig. 5.2b) shows the typical non-magnetic central line of austenite with an isomer shift of $\delta=-0.10(1) \mathrm{mm} / \mathrm{s}[94]$.

MOKE measurements as presented in Fig. 5.3 clearly demonstrate the ferromagnetic behavior of the ARMCO sputter target. A coercive field of $H_{C} \approx 75$ Oe is found. The observed magnetic inplane anisotropy is less than $1 \%$.

The angular dependence of the magnetic anisotropy as shown in Fig. 5.3 were deduced by 

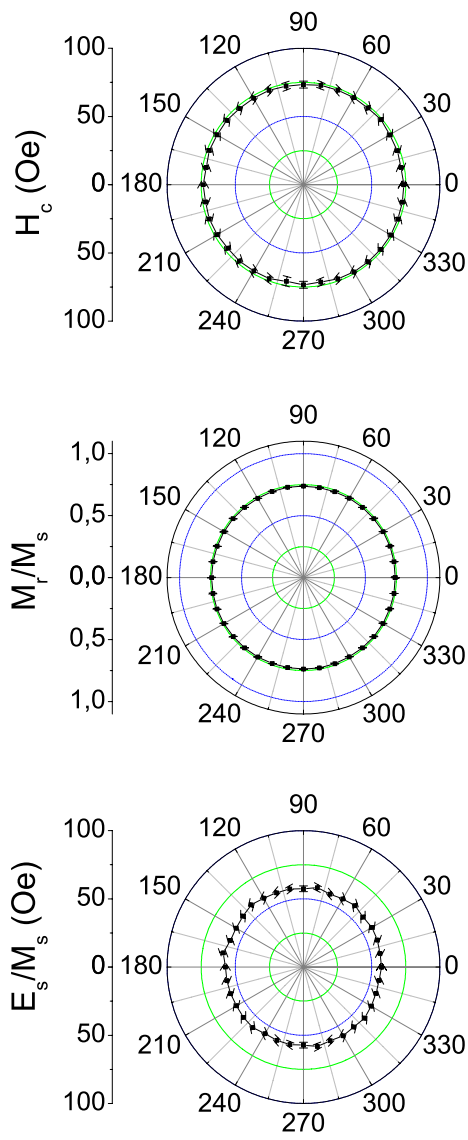

Figure 5.3: Angular scans of ARMCO-Iron sputter target: (top) coercitive field $H_{C}$; (middle) relative remanence $M_{r} / M_{s}$; (bottom) magnetization energy density $E_{s} / M_{s}$.

fitting the magnetization energy density $E_{s} / M_{s}$ required for saturation. The quantity of $E_{s} / M_{s}$ can be determinded by numerically integrating the anhysteretic magnetization loops averaged over both branches of the magnetization curves measured by MOKE [95], and was parameterized by the expression [96]:

$$
\begin{aligned}
\frac{E_{s}}{M_{s}} & =\frac{K_{u}}{M_{s}} \sin ^{2}\left(\varphi-\varphi_{0}\right) \\
& +\frac{K_{1}}{4 M_{s}} \sin ^{2} 2\left(\varphi-\varphi_{1}\right)+\frac{E_{0}}{M_{s}}
\end{aligned}
$$

where $E_{0} / M_{s}$ accounts for an isotropic contribution due to the random distribution of domains in the polycrystals. The parameters $K_{u} / M_{s}$ and $K_{1} / M_{s}$ indicate the contributions of uniaxial and foufold magnetic anisotropies with $\varphi_{u}$ and $\varphi_{1}$ being the respective symmetry angles.

The results of the fitting procedure can be obtained in Table 5.1.

Targets for Magnetron-sputtering possessed a diameter of $76 \mathrm{~mm}$ and $2 \mathrm{~mm}$ in thickness, targets for
Table 5.1: Fitting results of the MOKE analysis. $\chi^{2}$ and $\chi_{\text {red }}^{2}$ represent the values of the statistical significance tests.

\begin{tabular}{ll}
\hline$\chi^{2}$ & 0.640 \\
$\chi_{r e d}^{2}$ & 0.172 \\
$K_{u} / M_{s}$ & $0.860(372)$ \\
$K_{1} / 4 M_{s}$ & $1.616(1498)$ \\
$\varphi_{0}$ & $19.596(12444)$ \\
$\varphi_{1}$ & $75.500(13142)$ \\
$E_{0} / M_{s}$ & $56.986(288)$ \\
\hline
\end{tabular}

PLD possessed a dimension of $25 \times 25 \mathrm{~mm}$ and also $2 \mathrm{~mm}$ in thickness.

In the next chapters, the results of this work are presented and discussed. As a consequence of the facility of inspection, first, the results of the Magnetronsputtered films are shown, which are followed by the results of pulsed laser deposited films and by the results of FEL surface treated films. 


\section{Chapter 6}

\section{Results for magnetron-sputtered films}

\subsection{Results for magnetron- sputtered FeC films}

The films were sputter-deposited with an rf magnetron onto amorphous $\mathrm{SiO}_{2}$ substrates (oxidized $\mathrm{Si}(100)$ wafer of $0.5 \mathrm{~mm}$ thickness, pre-cleaned with acetone and oxidized in air, no further treatment) utilizing a pre-combined target consisting of graphite and commercial ARMCO-Iron (purity: $99.96 \%$ ). To achieve a stoichiometric FeC phase, the sputter yield of $\mathrm{Fe}$ and $\mathrm{C}$ was calculated using SRIM [97] in dependence to the incoming $\mathrm{Ar}^{+}$ion energy, which is illustrated in Fig. 6.1.

As obtained from Fig. 6.1, the sputter yield of

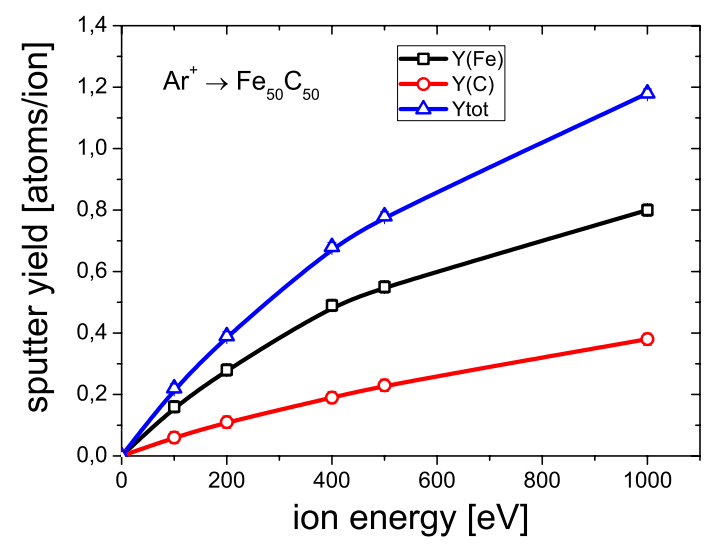

Figure 6.1: Sputtering-yield $Y$ of Fe, $\mathrm{C}$ and the sum of both elements in dependence of the $\mathrm{Ar}^{+}$ion energy.

Fe is 10 times greater than that of $\mathrm{C}$ at an $\mathrm{Ar}^{+}$ of approximately $80 \mathrm{eV}$. As a consequence, the target was made in that way, that the area fraction of graphite was 10 times greater than that of ARMCOIron. The form of the target can be readily seen in Fig. 6.2.

The target-substrate distance was set to $2 \mathrm{~cm}$ to

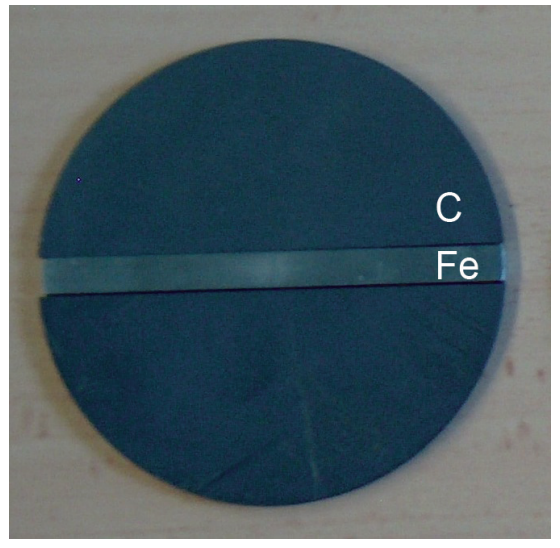

Figure 6.2: The $\mathrm{Fe}_{50} \mathrm{C}_{50}$ Magnetron sputter target.

achieve valuable sputter rates. The processing parameters were always $100 \mathrm{~W}$ magnetron power. The gas flow was increased from 14.5 to $140 \mathrm{sccm}$ ( $\mathrm{sccm}$ $=$ standard cubic centimeter, i.e. flow volume of gas at normal conditions, $273.15 \mathrm{~K}$ and $1013 \mathrm{hPa}$ ) during deposition. The sputter rate was always in the range of $0.01-0.03 \mathrm{~nm} / \mathrm{s}$, and was depending on the processing parameters. The magnetron chamber was evacuated to a base pressure of $10^{-4} \mathrm{~Pa}$ before deposition. The target was always pre-sputtered for half an hour with the desired parameters before starting the deposition onto the substrate. The target was water cooled and the substrate temperature was controlled between room temperature (water cooling) and $673 \mathrm{~K}$ (electrical heating). The thickness of the deposited films was controlled by a quartz microbalance (with a film density set to $4.15 \mathrm{~kg} / \mathrm{m}^{3}$, which is the density corresponding to the $\mathrm{Fe}$ and $\mathrm{C}$ ratio derived from the SRIM calculation). The samples were deposited with similar thicknesses as given by the quartz microbalance. As a result, deposition time and real film thickness varied. The deposition parameters and the resulting thicknesses and growth rates are summarized in 
Table 6.1

From these data it is derived that the growth rate

Table 6.1: Gas flow $j$, deposition time $t$, real film thickness $d$ (as measured by Rutherford Backscattering Spectrometry (RBS) after deposition) and derived growth rate $g=\frac{d}{t}$ for the deposited films. All samples were deposited at $298 \mathrm{~K}$ with a magnetron power of $100 \mathrm{~W}$ and a target-substrate dis-

\begin{tabular}{rrrrr} 
tance of $2 \mathrm{~cm}$. & \multicolumn{3}{c}{} \\
\cline { 2 - 4 } sample & $\begin{array}{r}j_{\text {Ar }} \\
{[\mathrm{sccm}]}\end{array}$ & $\begin{array}{r}t \\
{[\mathrm{~min}]}\end{array}$ & $\begin{array}{r}d \\
{[\mathrm{~nm}]}\end{array}$ & $\begin{array}{r}g \\
{[\mathrm{~nm} / \mathrm{min}]}\end{array}$ \\
\hline A145 & 14.5 & $145: 41$ & $64(10)$ & $0.44(7)$ \\
A300 & 30.0 & $102: 03$ & $119(10)$ & $1.17(10)$ \\
A400 & 40.0 & $233: 57$ & $45(10)$ & $0.19(4)$ \\
A500 & 50.0 & $90: 31$ & $39(10)$ & $0.98(11)$ \\
A600 & 60.0 & $101: 18$ & $99(10)$ & $0.98(10)$ \\
A700 & 70.0 & $85: 32$ & $83(10)$ & $0.97(12)$ \\
A800 & 80.0 & $80: 55$ & $107(10)$ & $1.32(13)$ \\
A1000 & 100.0 & $106: 43$ & $109(10)$ & $1.02(9)$ \\
A1400 & 140.0 & $107: 23$ & $102(10)$ & $0.95(9)$ \\
\hline
\end{tabular}

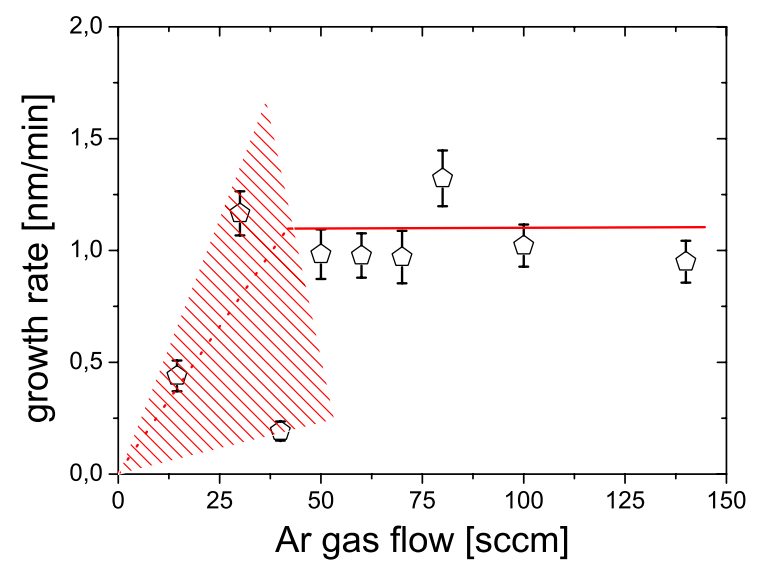

Figure 6.3: Growth rate $g$ as a function of the Ar gas flow $j_{\mathrm{Ar}}$ for the room temperature deposition.

is divided into two regions: the first region starts from 0 to $50 \mathrm{sccm}$ Ar gas flow, wherein the growth rate is oscillating (hatched area in Fig 6.3). This behavior could already be seen during deposition, where the formation of the plasma was hindered and where the processing had to be adjusted instantaneously. This behavior could be due to an enhanced plasma interaction caused by oxygen in the chamber. The second region follows the first up to $140 \mathrm{sccm}$, wherein the growth rate is constant at about $1.0 \mathrm{~nm} / \mathrm{min}$. It seems that, in this region, the energy of the $\mathrm{Ar}^{+}$atoms do not linearly correlate with the increase of the gas flow, but it is rather constant. As a consequence, no more target ions can be sputtered, which leads to a constant sputter rate. The evolution of the growth rate as a function of the Ar gas flow can be seen in Fig 6.3.

\subsubsection{Deposition at room tempera- ture and influence of the Ar gas flow}

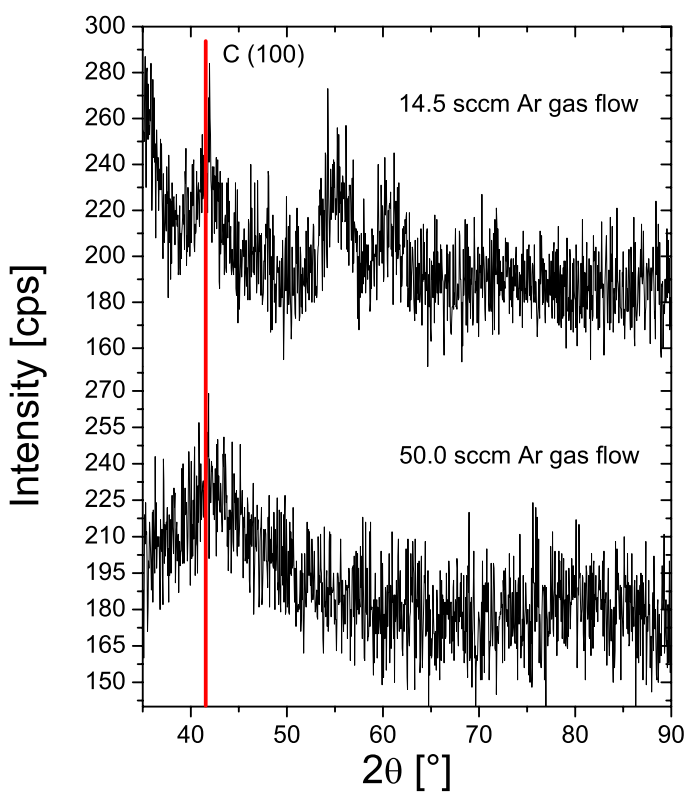

Figure 6.4: GIXRD $\left(2^{\circ}\right)$ spectra of the inert sputterd samples. The Ar gas flows are given in the graphs.

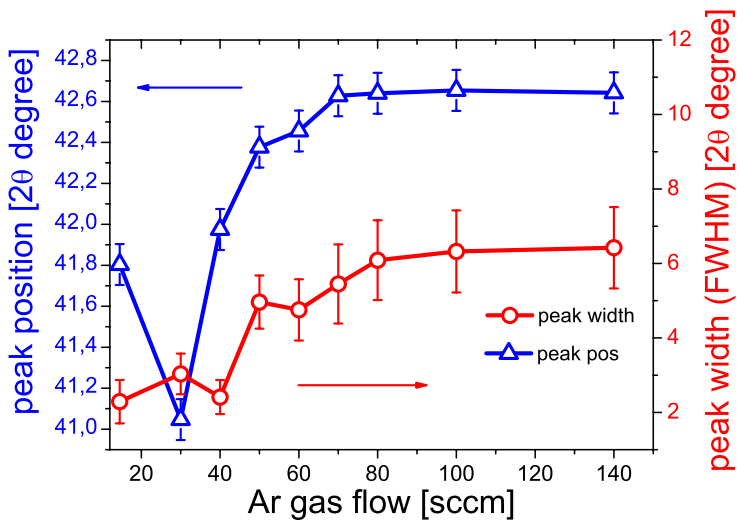

Figure 6.5: Peak position and peak width of the first broad peak in the XRD spectra of the reactive sputtered stainless steel films.

The Argon gas flow was systematically increased from 14.5 to $140.0 \mathrm{sccm}$. For all these deposited films, the inert magnetron deposited samples show the typical broad XRD appearance of amorphous materials. For the sake of clearness only the spectra of the samples sputtered with 14.5 and $50.0 \mathrm{sccm} \mathrm{Ar}$ gas flow are shown in Fig. 6.4. These diffractograms show a clear amorphous signature, but due to the high carbon content reflections of graphite are de- 

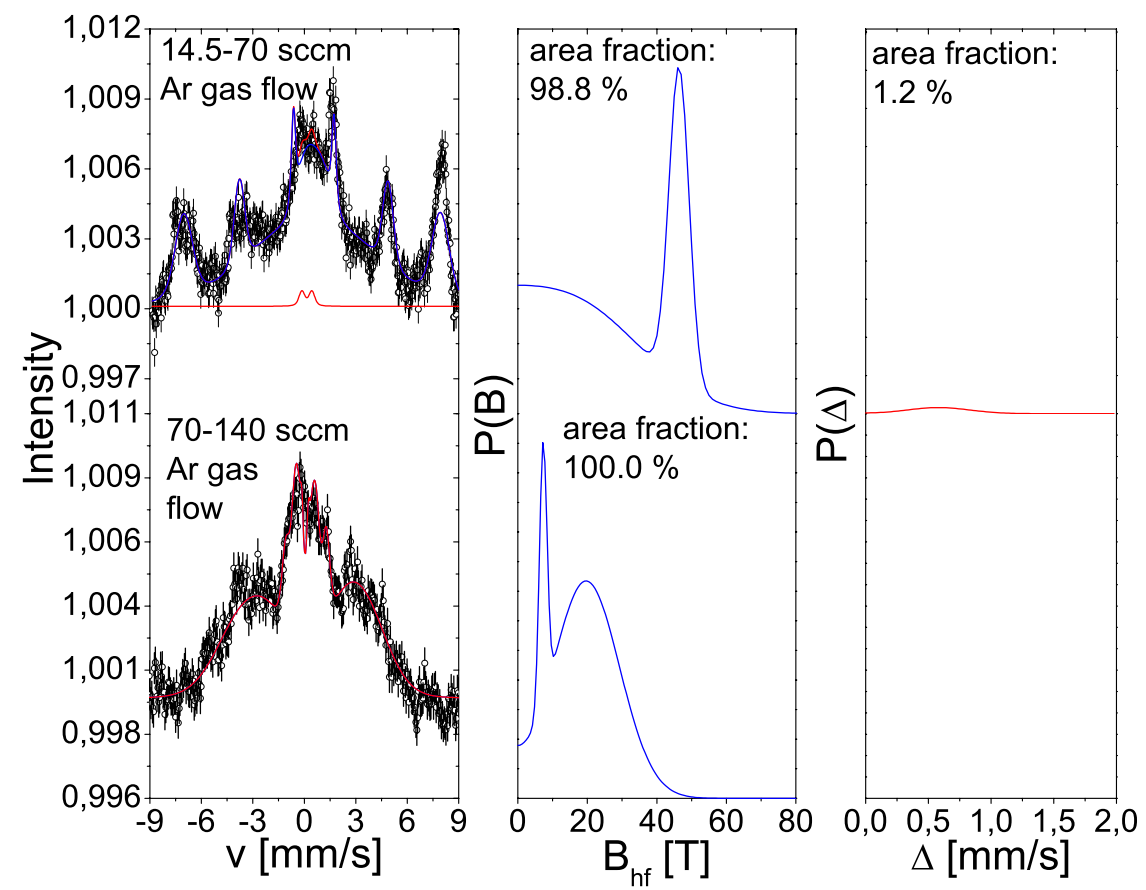

Figure 6.6: Mössbauer spectra of the inert sputtered FeC films.

Table 6.2: Mössbauer fitting results of the sputtered FeC samples, deposited at room temperature with a magnetron power of $100 \mathrm{~W}$ ( $f$ - area fraction (error), mean values of $\langle\delta\rangle$ - isomer shift, $\langle\Delta\rangle$ - the quadrupole splitting for the paramagnetic subspectra, $\langle\epsilon\rangle$ the quadrupole splitting for the magnetic subspectra, $B$ hyperfine field).

\begin{tabular}{|c|c|c|c|c|c|c|c|}
\hline $\begin{array}{c}\mathrm{Ar} \\
{[\mathrm{sccm}]}\end{array}$ & part & $\begin{array}{r}f \\
{[\%]}\end{array}$ & $\begin{array}{r}\langle\delta\rangle \\
{[\mathrm{mm} / \mathrm{s}]}\end{array}$ & $\begin{array}{l}\langle\Delta\rangle,\langle\epsilon\rangle \\
{[\mathrm{mm} / \mathrm{s}]}\end{array}$ & $\begin{array}{r}\sigma_{\Delta} \\
{[\mathrm{mm} / \mathrm{s}]}\end{array}$ & $\begin{array}{c}B\rangle \\
{[\mathrm{T}]}\end{array}$ & $\begin{array}{l}\sigma_{B} \\
{[\mathrm{~T}]}\end{array}$ \\
\hline \multirow[t]{3}{*}{$14.5-70.0$} & ferro-l & $32.2(6)$ & 0.05 & -0.03 & & $17.6(23)$ & $9.2(50)$ \\
\hline & ferro-h & $66.6(2)$ & 0.05 & -0.03 & & $46.4(3)$ & $2.9(3)$ \\
\hline & para & $1.2(5)$ & 0.15 & 0.58 & 0.24 & & \\
\hline \multirow[t]{2}{*}{$70.0-140.0$} & ferro-l & $11.8(27)$ & 0.03 & -0.04 & & $7.3(3)$ & $1.1(3)$ \\
\hline & ferro-h & $42.0(37)$ & 0.03 & -0.04 & & $19.7(5)$ & $9.6(6)$ \\
\hline
\end{tabular}

noted.

Fig. 6.5 shows the position and the width of the first broad peak for the spectra shown in Fig. 6.4.

There is the same clear tendency for peak position and peak width as obtained for the growth rate. For high Ar gas flows, peak position and peak width are nearly constant, indicating that no phase transition takes place.

Fig. 6.6 summarizes the CEMS measurements of the samples with increasing gas flow. They show the typical Mössbauer spectra of amorphous materials and are magnetically split. They also include distributions, which can be attributed to oxides. Especially, the samples sputtered between 14.5 and 70 sccm Ar gas flow contain high hyperfine fields which can be identified as $\alpha-\mathrm{Fe}_{2} \mathrm{O}_{3}$ oxides. The results of the fitting procedures are presented in Table 6.2 .
The spectra were fitted by hyperfine field distributions, and with quadrupole splitting distributions in the paramagnetic state, respectively.

For the FeC films sputtered between 70 and 140 sccm Ar gas flow, these distributions could be attributed to an amorphous iron carbon-alloy, possibly rich in carbon as indicated by ferro-l. Another possibility could be that the low fields in ferro-l are an artifact of the fitting procedure and correspond to paramagnetic doublets. In contrast to the low hyperfine fields, hyperfine fields as labeled as ferro$\mathrm{h}$ could be attributed to Fe carbides.

These Mössbauer results were confirmed by MOKE measurements. Due to the fact, that all samples show the same behavior in MOKE, exemplary the result of the $\mathrm{FeC}$ sample sputtered at $50 \mathrm{sccm}$ Ar gas flow is shown in Fig. 6.7. The coercive field $H_{C}$ of all samples is approximately $10 \mathrm{Oe}$. 


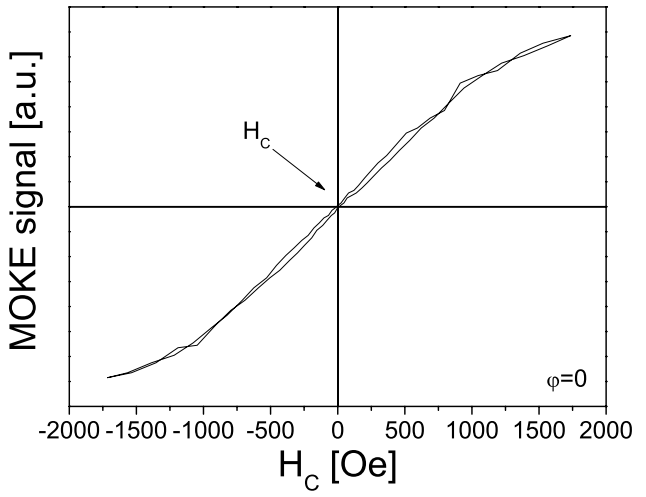

Figure 6.7: Hysteresis curve of the inert deposited FeC film sputtered at $50 \mathrm{sccm}$ Ar gas flow.

As seen in Fig. 6.7 the saturation is not reached. From the MOKE pattern it is estimated that a maximum external field of $0.30 \mathrm{~T}$ is needed to reach saturation. As a consequence, no angular dependencies could be determined. However, the coercitive field $H_{C}$ is not constant for different $\varphi$.

To characterize the surface of the samples, STM measurements were performed, as shown for the FeC sample deposited with an Ar gas flow of 140 sccm in Fig. 6.8.

For all films, a roughness of about $4 \mathrm{~nm}$ was

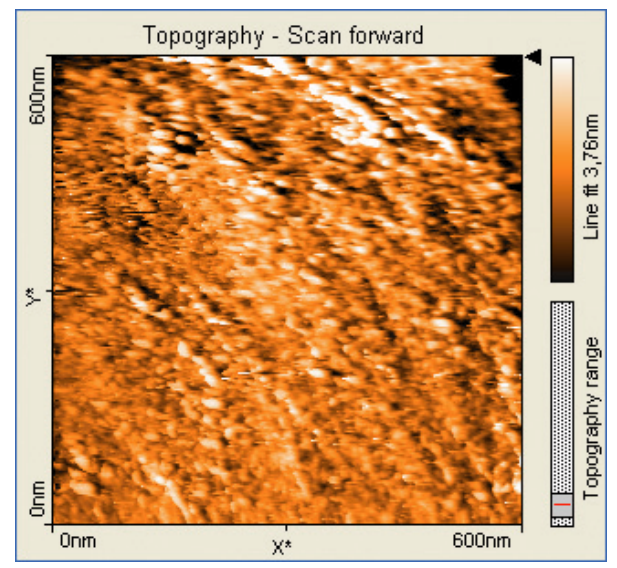

Figure 6.8: STM pattern for the FeC sample sputtered at $140 \mathrm{sccm}$ Ar gas flow.

found. To characterize the structure of the surface, an autocorrelation of the image was compiled and a power-spectral density (PSD) diagram derived which are illustrated in Fig. 6.9 and 6.10.

The structure obtained by the autocorrelation is not strongly minted, but a characteristic wavelength of $25 \mathrm{~nm}$ is derived by the PSD.

To determine the composition of the films, RBS measurements were carried out. As a consequence

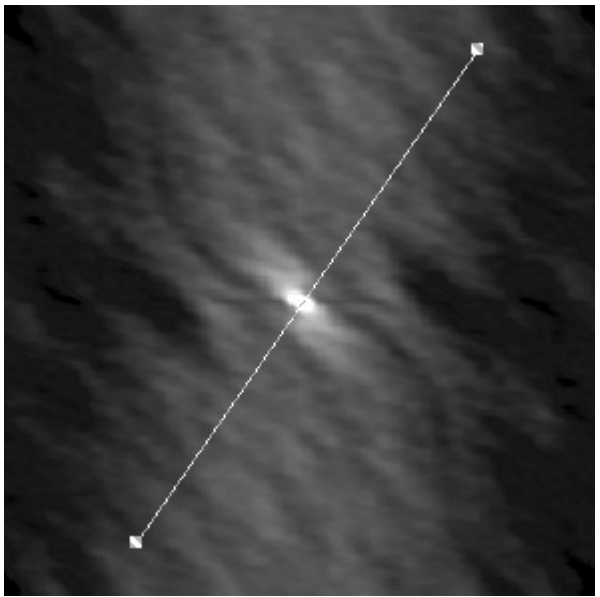

Figure 6.9: Autocorrelation of the STM image as shown in Fig. 6.8

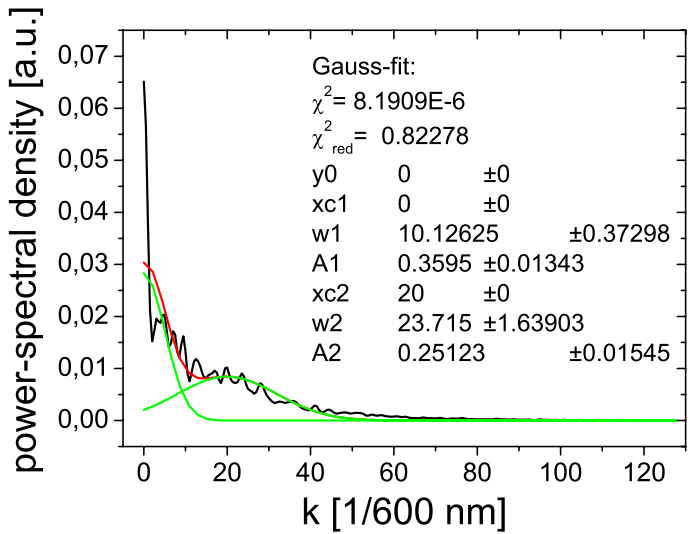

Figure 6.10: Power-spectral density diagram derived from the line scan, which is shown in Fig. 6.9. The parameters of the Gauss multi-peak fit are given in the graph.

of the low space between target and substrates, the films are very inhomogeneous, which is illustrates in Fig. 6.11.

Thus, more than 25 measurements were carried

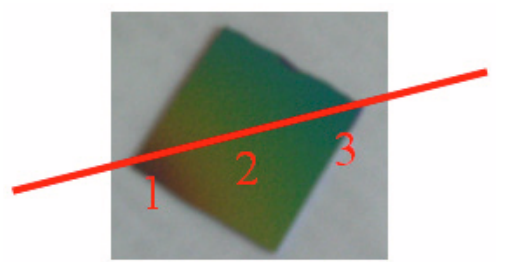

Figure 6.11: FeC film after deposition. The numbers indicate the positions of the RBS measurements.

out. For clarity, only the summarized results were 
presented in Fig. 6.12.

As obtained from the fitting, the FeC films also

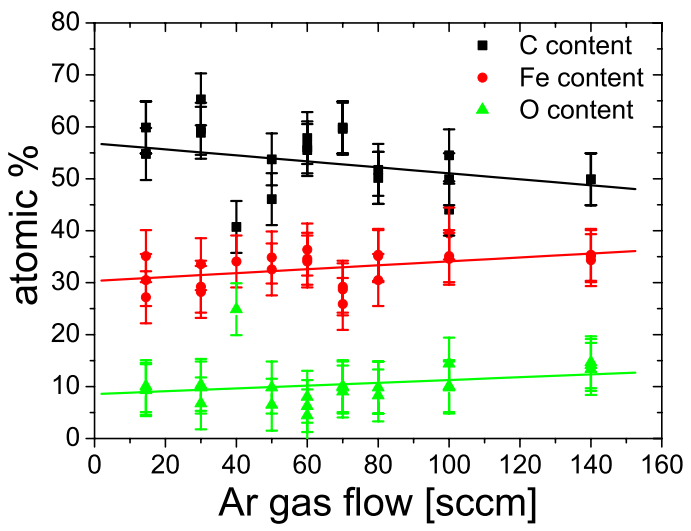

Figure 6.12: Mean concentrations as derived from the RBS fitting routine.

contain oxygen $(\approx 10 \%)$. As a result, the desired stoichiometric FeC film is not obtained. A closer look to the Richardson-Ellingham diagram reveals, that at those deposition pressures - such as used in this work - lead to the formation of iron oxides.

Another problem could be the poisoning of the target by reflected $\mathrm{C}$ ions or ions which are bombarding the target due to the selected target-geometry.

Fig. 6.13 illustrates the poisoning of the target as a function of the sputter yields of $\mathrm{Fe}$ and $\mathrm{C}$ at an fixed Ar ions energy of $400 \mathrm{eV}$. Thus, for any stoichiometries $\mathrm{Fe}_{100-C} \mathrm{C}_{C}$, the poisoning of the target can be deduced.

From this SRIM calculation a poisoning of nearly

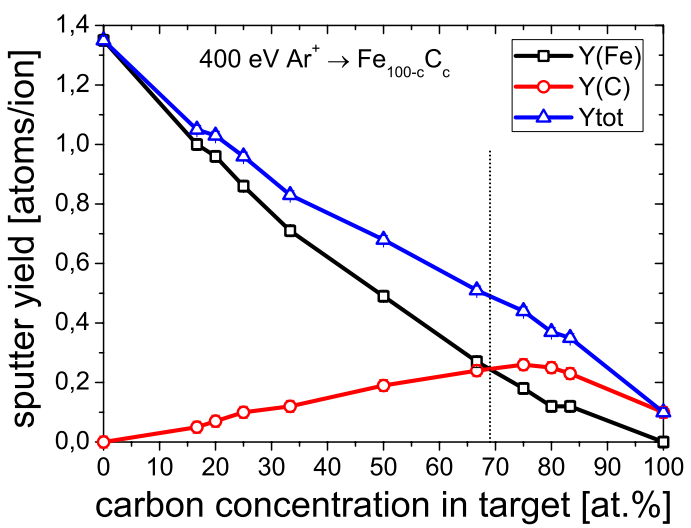

Figure 6.13: Poisoning of the target as a function of the sputter yields of $\mathrm{Fe}$ and $\mathrm{C}$ at fixed $E_{A r^{+}}=$ $400 \mathrm{eV}$. The black dashed line corresponds to the $\mathrm{Fe}_{50} \mathrm{C}_{50}$ stoichiometry.

$70 \%$ is predicted.

According to the RBS analysis, a large graphite

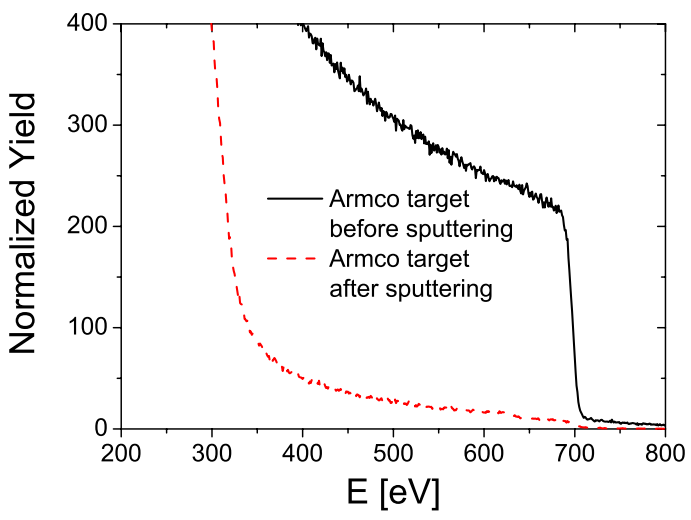

Figure 6.14: RBS spectra of the target before (black) and after (red dashed) sputtering.

layer on the surface of the target can be observed. This amount of graphite could lead to hysteresis effects, which hardly influence the stoichiometry of the deposited films.

As a consequence of this, the Magnetron sputtering technique seems not to be suitable to synthesize the stoichiometric FeC phase. Geometric effects of the target configuration, hysteresis effects (as indicated by RBS) and re-sputtering constitute severe problems to the deposition process. Even the small addition of $\mathrm{H}_{2}$ could not enhance the deposition process. Otherwise, Magnetron sputtering revealed the formation of films with amorphous character. These films exhibit carbon contents, which exceed the maximum solubility limits in known carbides. As a result of this, reactive sputtering of stainless steel - by using methane, nitrogen and oxygen as reactive gas - was performed to synthesize amorphous films or quasi metallic glasses based on conventional steels. The results will be discussed in the next sections. 


\subsection{Results for carburized stainless steel films}

The films were sputter-deposited with an rf magnetron onto amorphous $\mathrm{SiO}_{2}$ substrates (oxidized $\mathrm{Si}(100)$ wafer of $0.5 \mathrm{~mm}$ thickness, pre-cleaned with acetone and oxidized in air, no further treatment) utilizing a commercial AISI 316 (X5CrNiMo17-12$2,1.4401)$ target. The target-substrate distance was set to $10 \mathrm{~cm}$. The processing parameters were always $100 \mathrm{~W}$ magnetron power at a constant total gas flow of $12 \mathrm{sccm}$ ( $\mathrm{sccm}=$ standard cubic centimeter, i.e. flow volume of gas at normal conditions, $273.15 \mathrm{~K}$ and $1013 \mathrm{hPa}$ ) during deposition. Several sample series were deposited at different $\mathrm{CH}_{4}$ flows $(0.00-1.25 \mathrm{sccm})$. The sputter rate was always in the range of $0.1-0.2 \mathrm{~nm} / \mathrm{s}$, and was depending on the processing parameters. The magnetron chamber was evacuated to a base pressure of $10^{-4} \mathrm{~Pa}$ before deposition. The target was always pre-sputtered for half an hour with the desired parameters before starting the deposition onto the substrate. The target was water cooled and the substrate temperature was controlled between room temperature (water cooling) and $673 \mathrm{~K}$ (electrical heating). The thickness of the deposited films was controlled by a quartz microbalance (with a film density set to $7.89 \mathrm{~kg} / \mathrm{m}^{3}$, which is the density of AISI 316). The samples were deposited with similar thicknesses as given by the quartz microbalance. As a result, deposition time and real film thickness varied. The deposition parameters and the resulting thicknesses and growth rates are summarized in Table 6.3. From these data it is derived that

Table 6.3: Gas flow $j$, deposition time $t$, real film thickness $d$ (as measured by Rutherford Backscattering Spectrometry (RBS) after deposition) and derived growth rate $g=\frac{d}{t}$ for the deposited carburized films. All samples were deposited at $298 \mathrm{~K}$ with a magnetron power of $100 \mathrm{~W}$ and a target-

\begin{tabular}{cccccr} 
Substrate distance of 10 & $\mathrm{~cm}$. & & \\
\hline sample & $\begin{array}{c}j_{\mathrm{CH}_{4}} \\
{[\mathrm{sccm}]}\end{array}$ & $\begin{array}{c}j \mathrm{Ar} \\
{[\mathrm{sccm}]}\end{array}$ & $\begin{array}{c}t \\
{[\mathrm{~min}]}\end{array}$ & $\begin{array}{c}d \\
{[\mathrm{~nm}]}\end{array}$ & $\begin{array}{r}g \\
{[\mathrm{~nm} / \mathrm{min}]}\end{array}$ \\
\hline M0 & 0.00 & 12.00 & $17: 00$ & $205(10)$ & $12.06(92)$ \\
M1 & 0.01 & 11.99 & 19.49 & $242(10)$ & $12.21(84)$ \\
M5 & 0.05 & 11.95 & $20: 24$ & $220(10)$ & $10.78(74)$ \\
M10 & 0.10 & 11.90 & $19: 43$ & $238(10)$ & $12.07(83)$ \\
M50 & 0.50 & 11.50 & $27: 43$ & $208(10)$ & $7.50(46)$ \\
M75 & 0.75 & 11.25 & $31: 12$ & $216(10)$ & $6.92(39)$ \\
M100 & 1.00 & 11.00 & $33: 29$ & $222(10)$ & $6.63(36)$ \\
M125 & 1.25 & 10.75 & $37: 40$ & $267(10)$ & $7.09(33)$ \\
\hline
\end{tabular}

the growth rate is decreasing with increasing $\mathrm{CH}_{4}$ flow what is visualized in Fig. 6.15. The growth rate drops more or less exponentially from about $12 \mathrm{~nm} / \mathrm{min}$ for the inert sputtering to half of this value for higher methane flows. This might be due to the varying sputtering rates induced by the carbon uptake into the surface of the sputtering target.

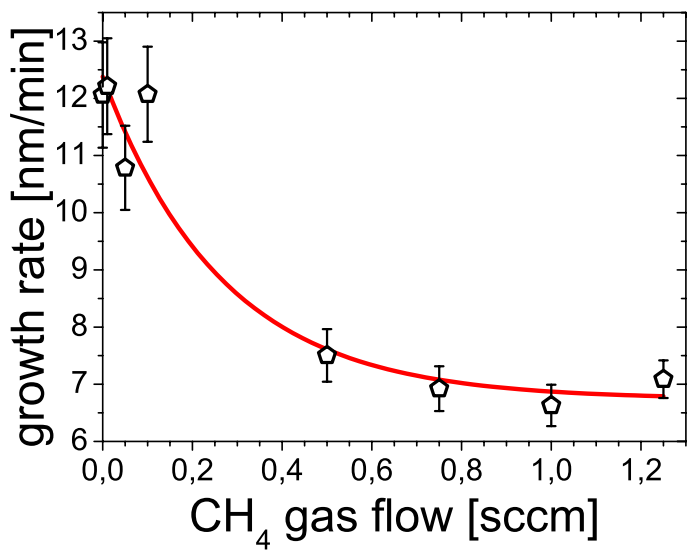

Figure 6.15: Growth rate $g$ as a function of the $\mathrm{CH}_{4}$ gas flow $j_{\mathrm{CH}_{4}}$ for the room temperature deposition.

\subsubsection{Deposition at room temper- ature and influence of the methane gas flow}

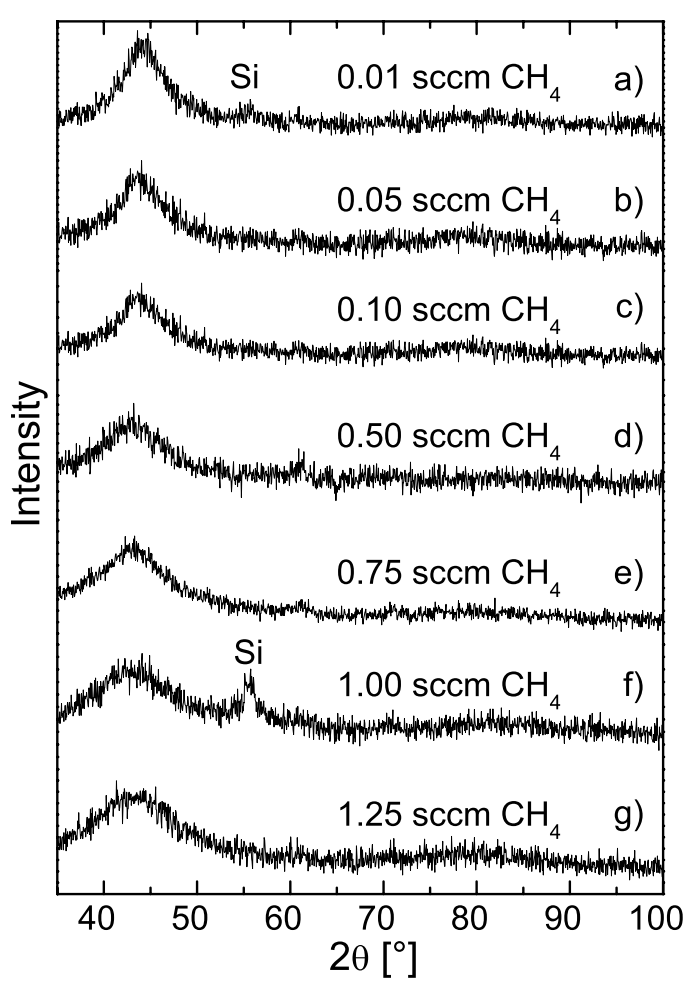

Figure 6.16: GIXRD $\left(2^{\circ}\right)$ spectra of the carburized films. The $\mathrm{CH}_{4}$ gas flows are given in the graph. 
Table 6.4: Mössbauer fitting results of the carburized AISI 316 samples, deposited at room temperature with a magnetron power of $100 \mathrm{~W}(f$ - area fraction (error), mean values of $\langle\delta\rangle$ - isomer shift, $\langle\Delta\rangle$ the quadrupole splitting for the paramagnetic subspectra, $\langle\epsilon\rangle$ the quadrupole splitting for the magnetic subspectra, $B$ - hyperfine field).

\begin{tabular}{ccrrrrrr}
\hline $\begin{array}{c}\mathrm{CH}_{4} \\
{[\mathrm{sccm}]}\end{array}$ & part & $\begin{array}{r}f \\
{[\%]}\end{array}$ & $\begin{array}{r}\langle\delta\rangle \\
{[\mathrm{mm} / \mathrm{s}]}\end{array}$ & $\begin{array}{r}\langle\Delta\rangle,\langle\epsilon\rangle \\
{[\mathrm{mm} / \mathrm{s}]}\end{array}$ & $\begin{array}{r}\sigma_{\Delta} \\
{[\mathrm{mm} / \mathrm{s}]}\end{array}$ & $\begin{array}{r}\langle B\rangle \\
{[\mathrm{T}]}\end{array}$ & $\begin{array}{r}\sigma_{B} \\
{[\mathrm{~T}]}\end{array}$ \\
\hline 0.01 & ferro-l & $84.0(3)$ & -0.04 & 0.03 & & $10.8(5)$ & $6.7(4)$ \\
& ferro-h & $12.4(15)$ & -0.04 & 0.03 & & $4.9(4)$ & $1.3(7)$ \\
& para & $3.6(3)$ & 0.15 & 0.58 & 0.24 & & \\
\hline 0.05 & ferro-l & $58.0(50)$ & -0.03 & -0.05 & & $23.2(24)$ & $4.8(12)$ \\
& ferro-h & $42.0(37)$ & -0.03 & -0.05 & & $12.7(55)$ & $5.7(23)$ \\
\hline 0.10 & ferro-l & $68.2(12)$ & 0.02 & -0.02 & & $27.8(5)$ & $4.1(5)$ \\
& ferro-h & $31.8(10)$ & 0.02 & -0.02 & & $15.0(6)$ & $7.9(35)$ \\
\hline 0.50 & ferro & $39.0(9)$ & 0.19 & 0.00 & & $9.7(4)$ & $2.5(9)$ \\
& para-1 & $31.2(10)$ & 0.14 & 0.40 & 0.20 & & \\
& para-2 & $29.8(11)$ & 0.16 & 0.93 & 0.20 & & \\
\hline 0.75 & ferro & $5.1(3)$ & 0.48 & 0.00 & & $24.3(9)$ & $1.0(2)$ \\
& para & $94.9(22)$ & 0.13 & 0.70 & 0.38 & & \\
\hline 1.00 & para & $100.0(1)$ & 0.14 & 0.69 & 0.38 & & \\
\hline 1.25 & para & $100.0(1)$ & 0.14 & 0.76 & 0.42 & & \\
\hline
\end{tabular}

The methane gas flow was systematically increased from $0.01 \mathrm{sccm}$ to $1.25 \mathrm{sccm}$. For all these deposited films, the reactive magnetron deposited and carburized samples show the typical broad XRD appearance of amorphous materials, as seen in Fig. 6.16. These diffractograms show a clear amorphous signature.

Fig. 6.17 shows the position and the width of the first broad peak for the spectra shown in Fig. 6.16. There is a clear tendency for peak position and peak width, where the peak position is decreasing and the peak width is increasing with increasing $\mathrm{CH}_{4}$ flow. Fig. 6.19 summarizes the CEMS measurements of
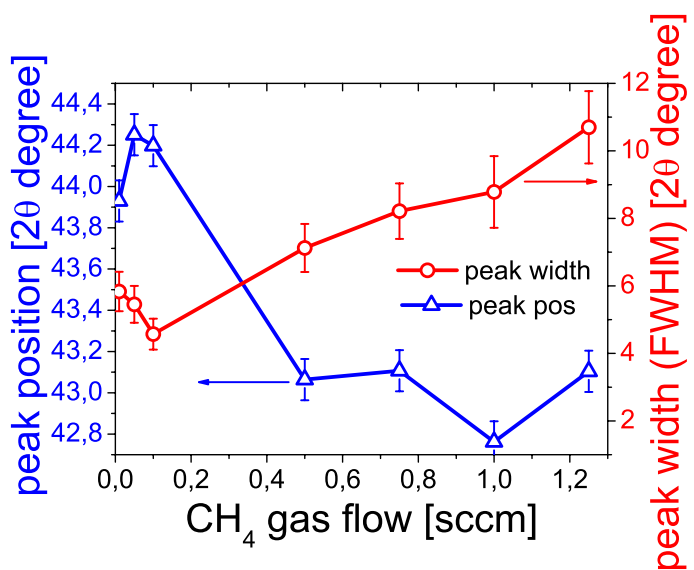

Figure 6.17: Peak position and peak width of the first broad peak in the XRD spectra of the reactive sputtered stainless steel films.

the samples with increasing gas flow. They show the typical Mössbauer spectra of amorphous materials. Spectra in a-c are magnetically split, those in e)-g) are non-magnetic, where the spectrum in d) shows both parts. This observation is consistent with the XRD results in Fig. 6.16. The results of the fitting procedures are presented in Table 6.4. The spectra were fitted by hyperfine field distributions, and with quadrupole splitting distributions in the paramagnetic state, respectively. These distributions are attributed to an amorphous stainless steel carbonalloy, possibly rich in carbon. The quadrupole splittings are broad and close to the values of the usual carbides. Not much information can therefore be taken from them. Summarizing, all samples appear as an amorphous material which shows magnetic behavior below a gas flow of $0.75 \mathrm{sccm}$.
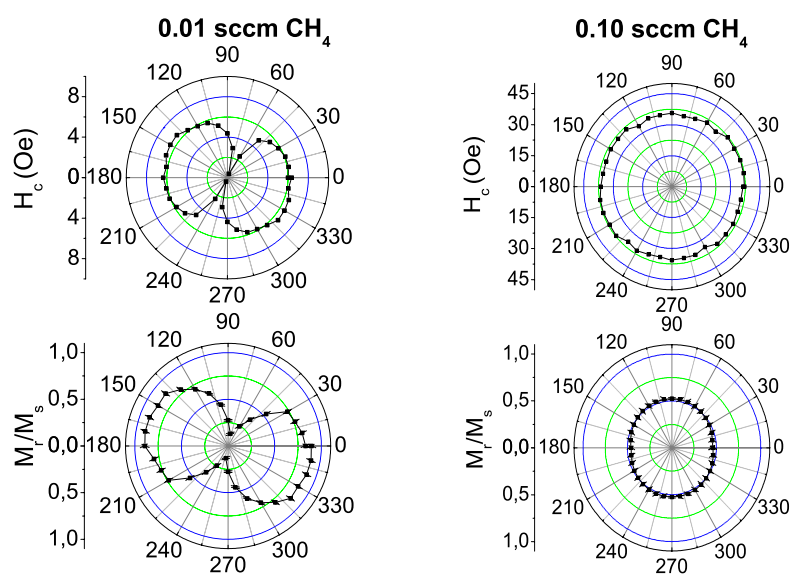

Figure 6.18: Angular scans of (left) $0.01 \mathrm{sccm} \mathrm{CH}_{4}$ and (right) $0.10 \mathrm{sccm} \mathrm{CH}_{4}$ gas flow. The coercive field $H_{\mathrm{C}}$ (top) and the relative remanence $M_{\mathrm{R}} / M_{\mathrm{S}}$ (bottom) are shown. 

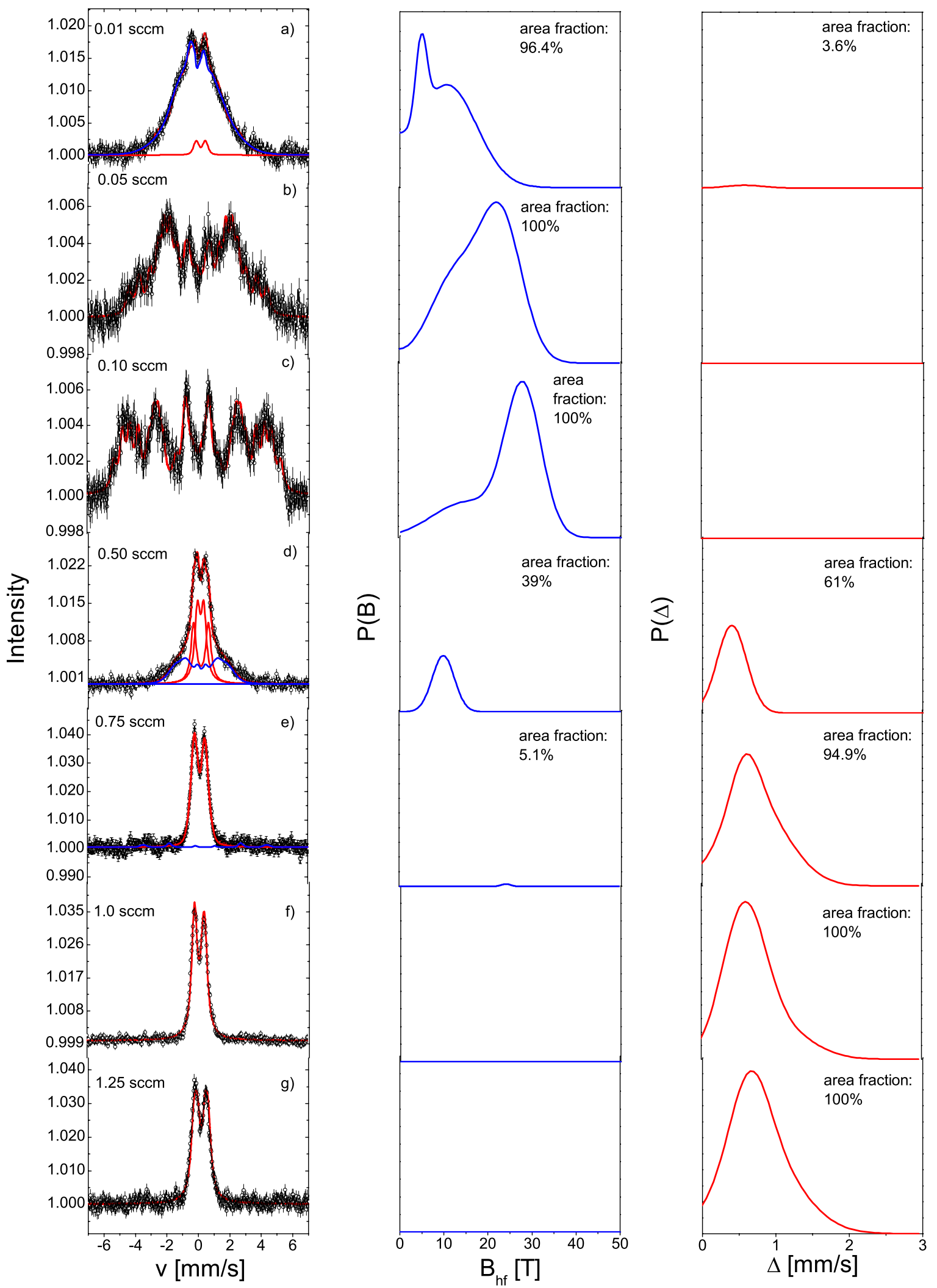

Figure 6.19: Mössbauer spectra of the reactive sputtered AISI 316 films. The numbers in the graphs represent the methane gas flow. 
These Mössbauer results were confirmed by MOKE measurements. Figure 6.18 shows the result of the MOKE measurement of the $0.01 \mathrm{sccm} \mathrm{CH}_{4}$ sample. The sample carburized with a gas flow of $0.05 \mathrm{sccm}$ $\mathrm{CH}_{4}$ shows an identical MOKE appearance.

A uniaxial anisotropy of about $70 \%$ can be seen with the maximum of the remanence around $150^{\circ}$. This behavior could be due to magnetostriction effects by stresses in the film. The coercive field $H_{C}$ of both samples is approximately 4 Oe. Figure $6.18 \mathrm{~b}$ ) shows the MOKE result of the as-carburized sample sputtered with $0.1 \mathrm{sccm} \mathrm{CH}_{4}$ gas flow. It exhibits only a weak (fourfold) anisotropy, which is normally observed for an fcc phase [89,98]. The coercive field was derived as 30 Oe. The samples with CEMS spectra shown in Fig. $6.19 \mathrm{~d}-\mathrm{g}$ ) do not show any magnetic behavior (e.g. no hysteresis loops in MOKE), which is in good agreement. These observations have now to be correlated to the carbon content of the film.

The EDX analyses of the deposited films revealed the original composition of the sputtering targets within the experimental limits. Unfortunately, the EDX did not allow to accurately determine elements lighter than oxygen. Therefore, in order to evaluate the amount of incorporated carbon, RBS measurements were carried out, whose results are shown in Fig. 6.20 .

The thickness of the films as obtained from the RBS analysis was already given in Table 6.3. The carbon concentration of the films as obtained from the RBS analysis is given in Fig. 6.21 and can reach almost 70 at.\%. There seems to be a clear correlation (parabolic-like) of the carbon content with the $\mathrm{CH}_{4}$ gas flow, with a minimum close to $25 \%$ carbon for $0.5 \mathrm{sccm} \mathrm{CH}_{4}$ flow. Unfortunately, RBS cannot distinguish between free carbon and carbon dissolved in the film. The C-content as achieved

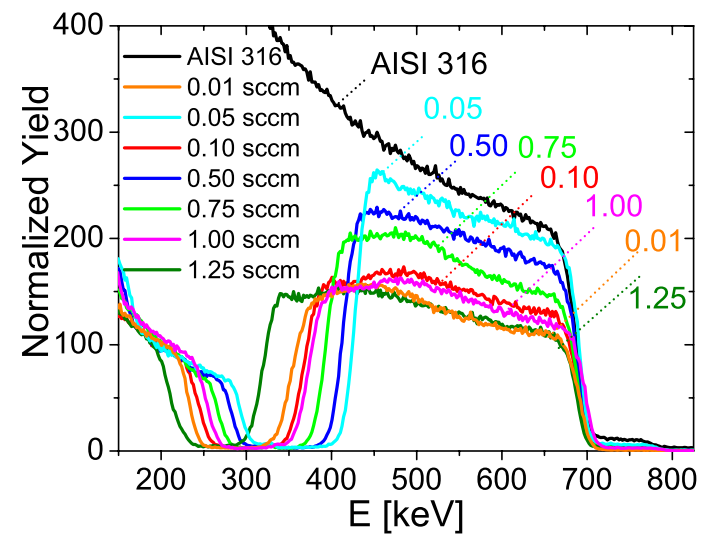

Figure 6.20: RBS spectra of the reactively sputtered films. The $\mathrm{CH}_{4}$ flow is given in the graph.

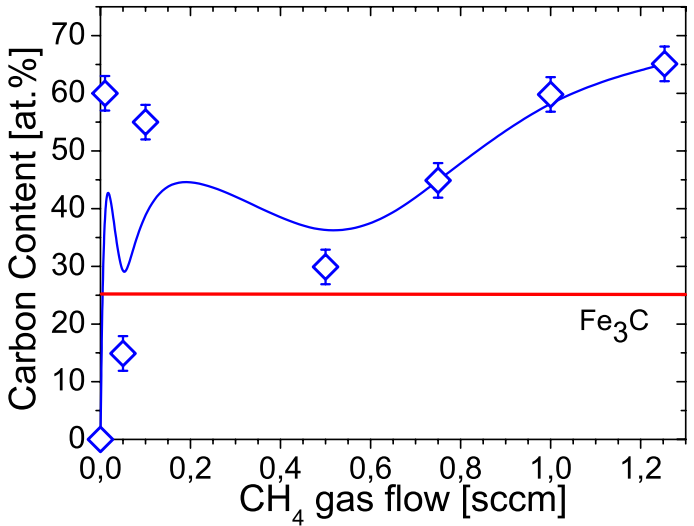

Figure 6.21: Carbon content in the reactively sputtered films as derived from the RBS analysis versus the $\mathrm{CH}_{4}$ gas flow.

by the RBS analyses exceeds the limit of 25 at. $\%$ for $\mathrm{Fe}_{3} \mathrm{C}$. Therefore, excessive carbon could eventually form graphite distributed in the film or on the surface of the film, but no signs of graphite could be detected in XRD. On the other hand, amorphous carbon would be difficult to see. To clarify this, AFM measurement were performed which is shown in Fig. 6.22 for the sample sputtered with a $\mathrm{CH}_{4}$ gas flow of $0.01 \mathrm{sccm}$. Nanoscaled surface struc-
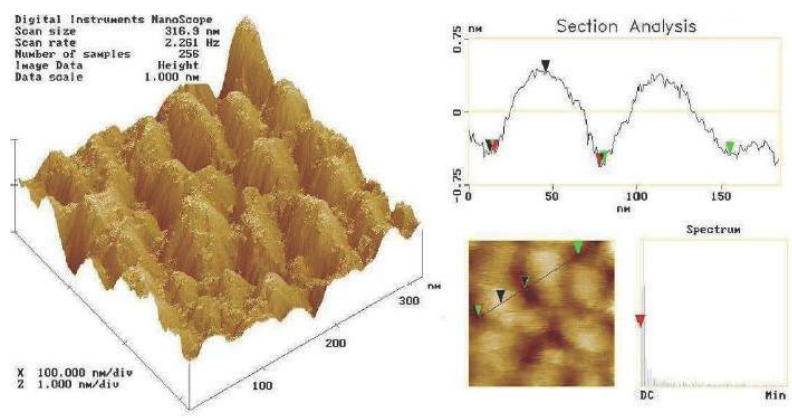

Figure 6.22: AFM measurement (left) and cross section analysis (right) of the sample sputtered with a $\mathrm{CH}_{4}$ gas flow of $0.01 \mathrm{sccm}$. The cross section analysis (right bottom) shows the line from which the roughness and the cluster-size were derived.

tures with the shape of half eggs can be observed. This shape is typical for carbon nanoclusters [99]. The clusters have a size between 10 and $80 \mathrm{~nm}$ in diameter and the roughness of the film is $R_{a}=3 \mathrm{~nm}$. This method is not able to show the type of chemical bonding of the carbon clusters. Thus, the carburized samples were examined by means of Raman spectroscopy, which is displayed in Fig. 6.23. The Raman results show a narrow weak peak at a Raman shift of $1550 \mathrm{~cm}^{-1}$ for the soft ferromag- 


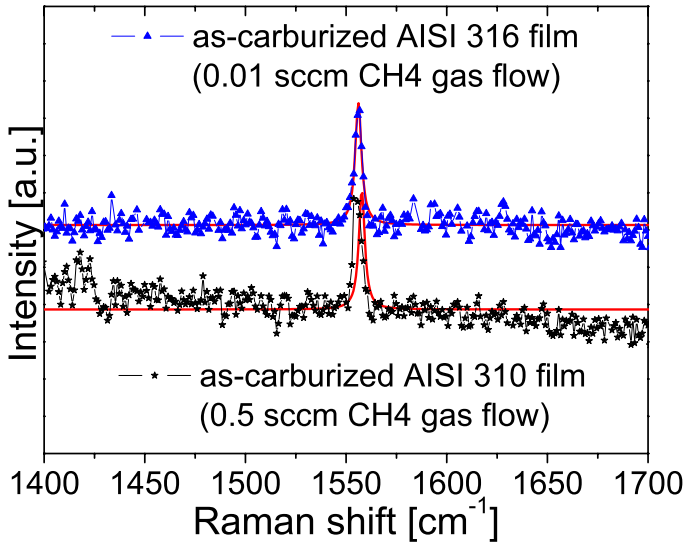

Figure 6.23: Raman results for the amorphous soft ferromagnetic carburized AISI 310 and AISI 316 films.

netic phase found in carburized thin AISI 316 (at $0.01 \mathrm{sccm} \mathrm{CH}_{4}$ gas flow) and AISI 310 (at $0.05 \mathrm{sccm}$ $\mathrm{CH}_{4}$ gas flow) films. In literature [100], this shift has been attributed to the carbon g-band ( $\mathrm{sp}^{2}$ hybridized C). No d-band is observed around 1300$1350 \mathrm{~cm}^{-1}$. The low intensity and the narrow peak form is typical for nanoparticles. This is a hint that at least some carbon forms graphite nanoparticles at the surface. One can compare the overall carbon ratios of the different films. These are in good agreement with the RBS results. According to the method described in [101], the stress in the film could be derived on the basis of the Raman peak positions, which add up to 2.5 - 3.3 GPa. Note, no clear dependencies can be seen between the stress in the film and composition of the target material.

\subsubsection{Vacuum annealing of films car- burized at room temperature}

In order to analyze the thermal stability of the amorphous soft ferromagnetic phase, we performed annealing at a temperature of $973 \mathrm{~K}$. The exposition times were set to 1,10 and $120 \mathrm{~h}$. The Mössbauer results of this isothermal series are shown in Fig. 6.24.

The Mössbauer spectra show various subspectra which can be attributed to carbides. The observed carbides and their fractions support the carbide formation upon annealing and hint to the carbide transformation $\mathrm{M}_{3} \mathrm{C} / \mathrm{M}_{7} \mathrm{C}_{3} \rightarrow \mathrm{M}_{23} \mathrm{C}_{6} \rightarrow \mathrm{M}_{6} \mathrm{C}$ with increasing exposition time [102]. The Mössbauer results are summarized in Table 6.5. The hyperfine parameters after annealing for $1 \mathrm{~h}$ are well-defined and the quadrupole doublets can be identified as $\mathrm{M}_{3} \mathrm{C} / \mathrm{M}_{7} \mathrm{C}_{3}$ carbides $[103,104]$. The observed $\alpha$-Fe

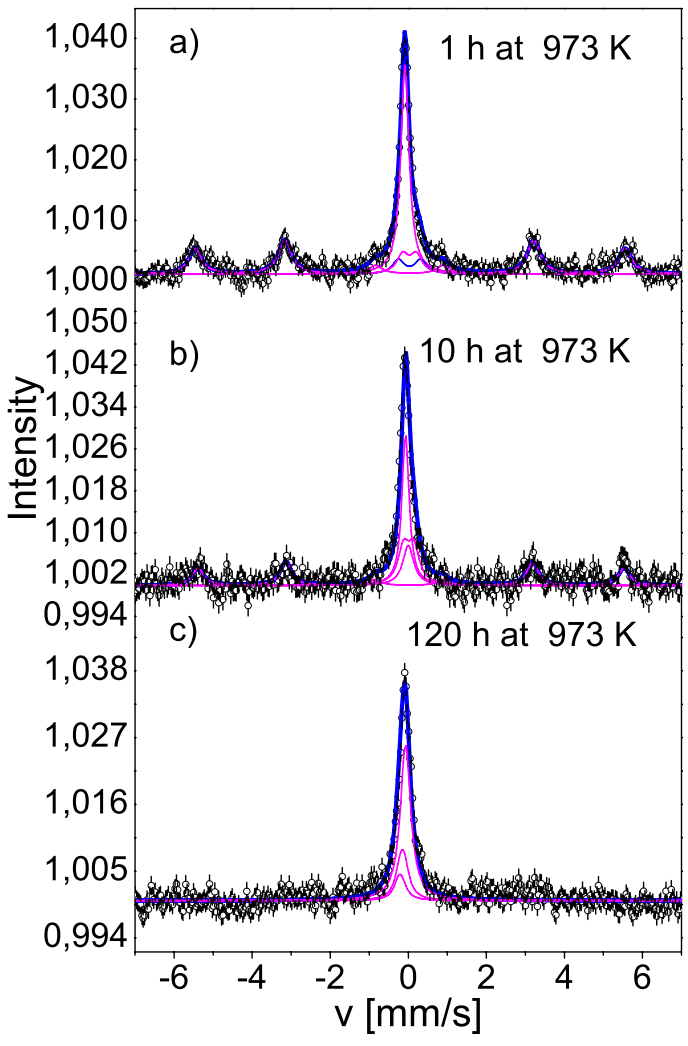

Figure 6.24: Mössbauer spectra of vacuum annealed AISI 316 film sputtered with a magnetron power of $100 \mathrm{~W}$ and 0.01 sccm methane. Exposition time and temperature are given.

sextet can be interpreted as bcc Fe probably containing some Ni, but almost no $\mathrm{Cr}$ and Mo [105]. After $10 \mathrm{~h}$ annealing, the doublets were identified as $\mathrm{M}_{23} \mathrm{C}_{6}$ according to Refs. $[103,106]$ and an $\alpha$-Fe sextet as before. After $120 \mathrm{~h}$ vacuum annealing, the doublets can be identified as $\mathrm{M}_{6} \mathrm{C}$ [107]. The corresponding XRD patterns are shown in Fig. 6.25.

The XRD pattern are in good agreement with the Mössbauer results. The mixed carbides $\mathrm{M}_{7} \mathrm{C}_{3} / \mathrm{M}_{23} \mathrm{C}_{6} / \mathrm{M}_{6} \mathrm{C}$ are observed. In consideration of these data, the transformations during annealing can be understood as follows $[103,104,108,109]$ : carbon diffuses out of the amorphous matrix and forms Cr-rich carbides, which further enriches in $\mathrm{Cr}$ and further transforms with increasing exposition time. The remaining matrix is more and more enriched in $\mathrm{Ni}$ and then transforms to $\gamma$. The $\alpha$-Fe(110) peak and the $\mathrm{M}_{7} \mathrm{C}_{3}$-peak are overlapping, first this peak consists mostly of $\alpha$-Fe which is then more and more transformed into $\mathrm{M}_{7} \mathrm{C}_{3}$. In the bottom-most XRD pattern it is only $\mathrm{M}_{7} \mathrm{C}_{3}$. Any supersaturated dissolved carbon tries to segregate and is used for carbide formation. A closer look to the stoichiometry shows an decrease of the C-content in the car- 
Table 6.5: Mössbauer results for the vacuum annealed sample deposited with 0.01 sccm methane flow $(\delta$ - isomer shift, $\Delta$ - the quadrupole splitting for the paramagnetic subspectra, $\epsilon$ the quadrupole splitting for the magnetic subspectra, $B$ - hyperfine field, $\Gamma$ - line width (HWHM), $f$ - area fraction).

\begin{tabular}{ccccccc}
\hline $\begin{array}{c}\text { Annealing time } \\
{[\mathrm{h}]}\end{array}$ & subspectrum & $\begin{array}{c}\delta \\
{[\mathrm{mm} / \mathrm{s}]}\end{array}$ & $\begin{array}{c}\Delta, \epsilon \\
{[\mathrm{mm} / \mathrm{s}]}\end{array}$ & $\begin{array}{c}B \\
{[\mathrm{~T}]}\end{array}$ & $\begin{array}{c}\Gamma \\
{[\mathrm{mm} / \mathrm{s}]}\end{array}$ & $\begin{array}{c}f \\
{[\%]}\end{array}$ \\
\hline 1 & $\gamma$ & $-0.08(2)$ & & & $0.12(8)$ & $35.2(1)$ \\
& $\mathrm{M}_{3} \mathrm{C} / \mathrm{M}_{7} \mathrm{C}_{3}$ & $0.03(5)$ & $0.35(4)$ & & $0.18(2)$ & $12.0(4)$ \\
& $\mathrm{M}_{3} \mathrm{C} / \mathrm{M}_{7} \mathrm{C}_{3}$ & $0.04(5)$ & $0.57(4)$ & & $0.17(8)$ & $12.2(5)$ \\
$\alpha-\mathrm{Fe}(\mathrm{Ni})$ & $0.04(5)$ & $0.09(5)$ & $34.2(2)$ & $0.20(3)$ & $40.6(6)$ \\
\hline 10 & $\gamma$ & $-0.074(5)$ & & & $0.13(10)$ & $28.7(4)$ \\
& $\mathrm{M}_{23} \mathrm{C}_{6}$ & $0.00(5)$ & $0.17(8)$ & & $0.18(12)$ & $16.6(5)$ \\
& $\mathrm{M}_{23} \mathrm{C}_{6}$ & $0.01(1)$ & $0.26(2)$ & & $0.18(8)$ & $22.2(3)$ \\
& $\alpha-\mathrm{Fe}(\mathrm{Ni})$ & $0.04(2)$ & $0.02(4)$ & $33.8(4)$ & $0.17(6)$ & $32.5(8)$ \\
\hline 120 & $\gamma$ & $-0.07(11)$ & & & $0.16(1)$ & $66.3(4)$ \\
& $\mathrm{M}_{6} \mathrm{C}$ & $-0.21(11)$ & $0.08(4)$ & & $0.15(7)$ & $11.3(2)$ \\
& $\mathrm{M}_{6} \mathrm{C}$ & $-0.15(7)$ & $0.10(5)$ & & $0.15(11)$ & $22.4(4)$ \\
\hline
\end{tabular}

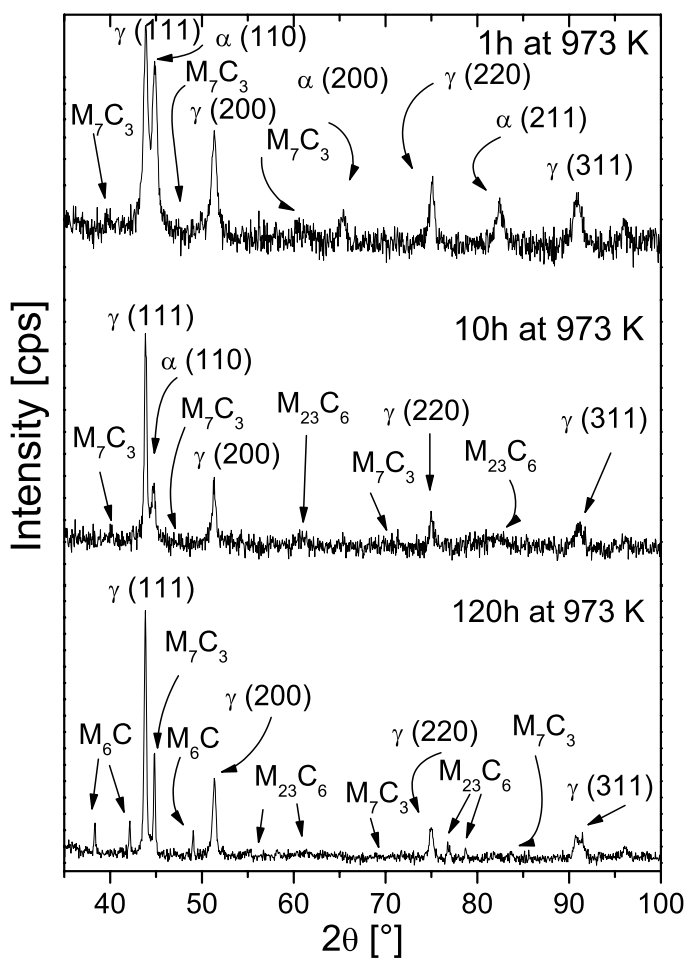

Figure 6.25: GIXRD ( $2^{\circ}$ incidence angle) spectra of the post-vacuum annealed samples sputtered with a magnetron power of $100 \mathrm{~W}$ and 0.01 sccm methane. Annealing temperature and time are given.

bides with annealing time, from $\mathrm{M}_{7} \mathrm{C}_{3}$ over $\mathrm{M}_{23} \mathrm{C}_{6}$ to $\mathrm{M}_{6} \mathrm{C}$.

SEM pictures show that the excessive $\mathrm{C}$ diffuses also towards the surface and there forms nanowires, as seen in Fig. 6.26. In addition, Raman spectroscopy was used to examine the bond behavior of the carbon after annealing. The spectra are shown in Fig. 6.27.

Both $\mathrm{sp}^{2}$ - and $\mathrm{sp}^{3}$-hybridized carbon is observed. According to the three stage model of Ferrari et

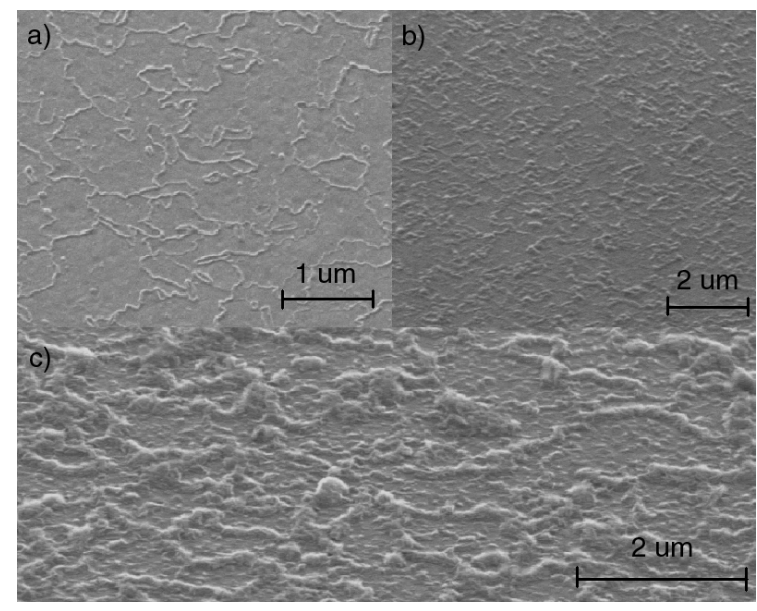

Figure 6.26: SEM pictures of the post-vacuum annealed samples: a) $1 \mathrm{~h}$ at $973 \mathrm{~K}$, b) $10 \mathrm{~h}$ at $973 \mathrm{~K}$ and c) $120 \mathrm{~h}$ at $973 \mathrm{~K}$.

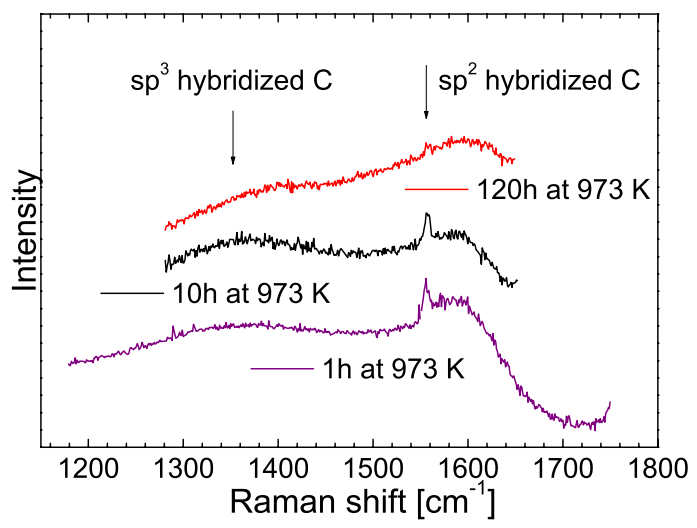

Figure 6.27: Raman measurements of the postvacuum annealed samples.

al. $[110]$

$$
I_{D} / I_{G}=C^{\prime}(\lambda) \cdot L^{2}{ }_{a}
$$


where $\mathrm{I}_{D}$ and $\mathrm{I}_{G}$ are the intensities of the $\mathrm{D}$ and $\mathrm{G}$ peak, $\mathrm{C}$ the Raman coupling coefficient $\left(C^{\prime}(514.5 \mathrm{~nm})=0.0055\right)$ and $L_{a}$ the cluster size, the $\mathrm{sp}^{2} / \mathrm{sp}^{3}$ ratio can be derived and thus the cluster size, which is in good agreement to AFM measurements. The results are given in Table 6.6. Finally,

Table 6.6: Raman results of the vacuum-annealed samples

\begin{tabular}{ccc}
\hline $\begin{array}{c}\text { Annealing time } \\
\text { at } 973 \mathrm{~K}[\mathrm{~h}]\end{array}$ & $\mathrm{I}_{D} / \mathrm{I}_{G}$ & $\begin{array}{c}\mathrm{L}_{a} \\
{[\mathrm{~nm}]}\end{array}$ \\
\hline 1 & $0.53(3)$ & $10(1)$ \\
10 & $0.85(2)$ & $12(2)$ \\
120 & $0.75(1)$ & $12(1)$ \\
\hline
\end{tabular}

the samples can be embedded in the ternary phase diagram of amorphous carbon, which can be attributed to sputtered a-C. The weak and narrow peak at $1550 \mathrm{~cm}^{-1}$ again implies nanoclustering of graphite on the surface of the samples.

\subsubsection{The influence of deposition temperature}

In Fig. 6.28 the CEM spectra of carburized AISI 316 films deposited at increasing substrate temperatures are shown. The deposition temperature clearly changes the nature of the deposited films, as seen by the changes in the spectra. It seems that at higher temperatures, the low $B$ region has transformed in a non-magnetic quadrupole distribution. RBS show a decrease of carbon content from $60(2) \%$ to $30(2) \%$, which affects the XRD pattern, in which a more clearer formation of the $\gamma$-phase is observed. The decrease of the carbon content originates in the formation of carbon-oxides or hydroxides, which shade again into the vapor phase. The Mössbauer results are given in Table 6.7.

Consequently, the long-range diffusion process and thus the suppression of nucleation are less severe at higher deposition temperatures. In Fig. 6.29 the XRD diffraction patterns are shown, the bars indicate the Bragg positions of the different reflexes as indicated.

At $298 \mathrm{~K}$ the amorphous phase dominates. At $673 \mathrm{~K}$, a partial recrystallization process can be observed by the formation of carbides, but the amorphous character of the sample is maintained. Discrepancies in the peak positions imply the presence of stress in the films.

The results for the deposition temperature of $473 \mathrm{~K}$ were not reproducible, especially the Mössbauer spectra showed different grades of oxidation. This could be due to an enhanced plasma interaction between $\mathrm{CH}_{4}$-radicals and oxygen. As a consequence of this, these results were not shown. Fig. 6.30
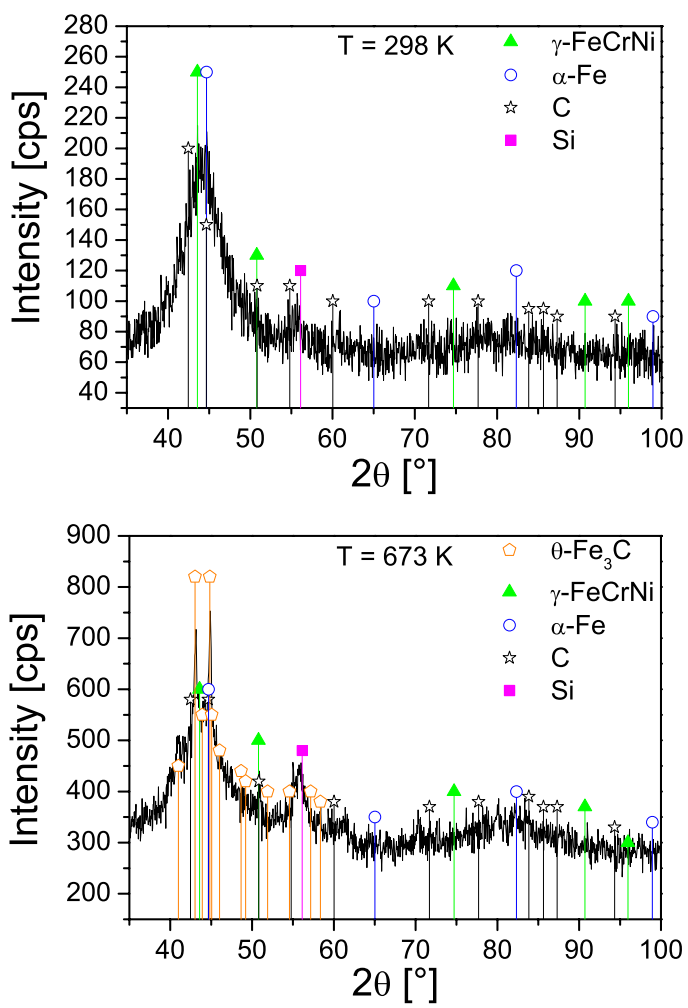

Figure 6.29: GIXRD ( $2^{\circ}$ incidence angle) spectra of the as-carburized samples sputtered with a magnetron power of $100 \mathrm{~W}$ and $0.01 \mathrm{sccm}$ methane. The substrate temperatures are given.

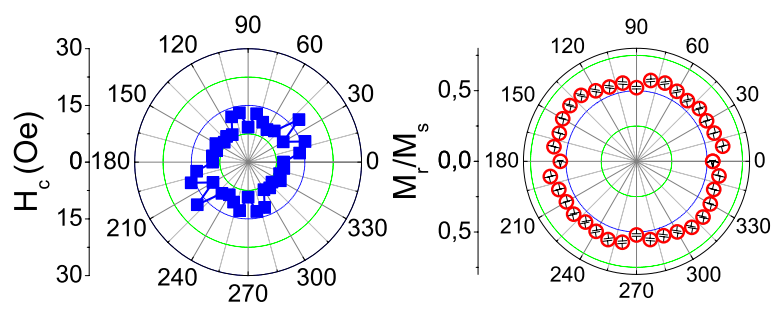

Figure 6.30: Angular scans of the film sputtered at $673 \mathrm{~K}$ with $0.1 \mathrm{sccm} \mathrm{CH}_{4}$ gas flow. The polar diagrams of the coercive field $H_{\mathrm{C}}$ ( left) and the relative remanence $M_{\mathrm{R}} / M_{\mathrm{S}}$ (right) are shown.

shows the MOKE measurement of the sample sputtered at $673 \mathrm{~K}$. A weak fourfold anisotropy can be observed, which is typical for fcc phases [111]. The coercive field is about 6 Oe. This is in good agreement with the CEMS and GIXRD results. Deposition temperature is not as critical in phase formation as shown in inert sputtered AISI 316 stainless-steel films. The amorphous soft ferromagnetic phase mostly remains. 

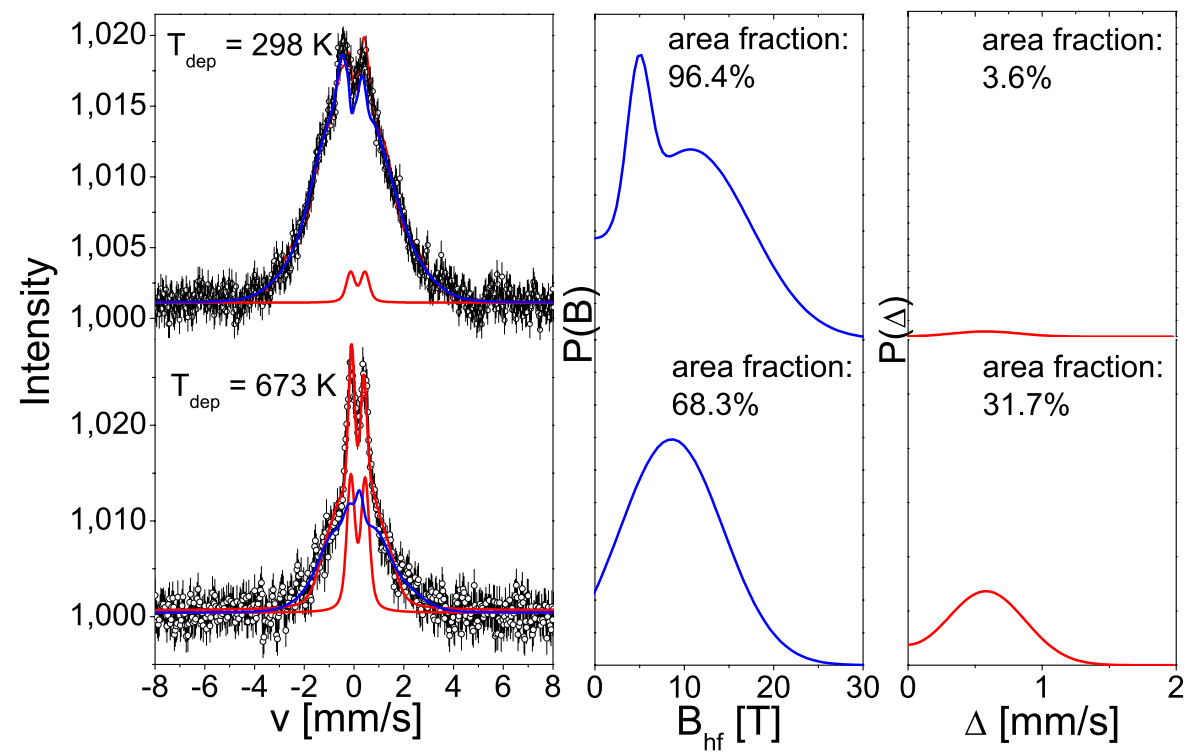

Figure 6.28: CEM spectra of carburized AISI 316 films sputtered with a magnetron power of $100 \mathrm{~W}$ and $0.01 \mathrm{sccm}$ methane at two temperatures: $\mathrm{T}=298 \mathrm{~K}$ (top), $\mathrm{T}=673 \mathrm{~K}$ (bottom). On the right hand side, the hyperfine field distribution $p(B)$ and the quadrupole splitting distribution $p(\Delta)$ are shown.

Table 6.7: CEM results of AISI 316 films, deposited at $673 \mathrm{~K}$ with a magnetron power of $100 \mathrm{~W}$ and $0.01 \mathrm{sccm} \mathrm{CH}_{4}$ flow ( $f$ - area fraction (error), mean values of $\delta$ - isomer shift, $\langle\Delta\rangle$ - the quadrupole splitting for the paramagnetic subspectra, $\langle\epsilon\rangle$ the quadrupole splitting for the magnetic subspectra, $B$ hyperfine field).

\begin{tabular}{ccrrrrrr}
\hline $\begin{array}{c}\mathrm{CH}_{4} \\
{[\mathrm{sccm}]}\end{array}$ & part & $\begin{array}{r}f \\
{[\%]}\end{array}$ & $\begin{array}{r}\langle\delta\rangle \\
{[\mathrm{mm} / \mathrm{s}]}\end{array}$ & $\begin{array}{r}\langle\Delta\rangle,\langle\epsilon\rangle \\
{[\mathrm{mm} / \mathrm{s}]}\end{array}$ & $\begin{array}{r}\sigma_{\Delta} \\
{[\mathrm{mm} / \mathrm{s}]}\end{array}$ & $\begin{array}{r}\langle B\rangle \\
{[\mathrm{T}]}\end{array}$ & $\begin{array}{r}\sigma_{B} \\
{[\mathrm{~T}]}\end{array}$ \\
\hline 0.01 & ferro & $68.3(4)$ & 0.17 & 0.12 & & $8.6(2)$ & $5.7(2)$ \\
& para-1 & $29.2(2)$ & 0.16 & 0.59 & 0.28 & & \\
& para-2 & $0.4(2)$ & -0.10 & 0.10 & 0.04 & & \\
& para-3 & $2.1(3)$ & 0.17 & 0.50 & 0.04 & & \\
\hline
\end{tabular}

\subsubsection{Corrosion tests of carburized stainless steel films}

In order to investigate the influence of the carburization on corrosion resistance, corrosion tests were performed on carburized stainless steel films, whose results are presented in Fig. 6.31. A comparison of the two graphs provides the following differences between AISI 310 and AISI 316: the first peak at $-1.3 \mathrm{~V}$ can be attributed to surface-diffused $\mathrm{Cr}$. Note, the intensities in both steels are different due to the frequency of occurrence of the alloying elements. AISI 316 shows a better corrosion resistance in the passive area. In the range of -1 to $-0.25 \mathrm{~V}$ it achieves negative current densities. Negative current densities are useful for the formation of oxides at the surface which increases the corrosion resistance. AISI 310 holds more Cr than AISI 316, but its current density rises again at $-0.6 \mathrm{~V}$. This can be attributed to the Ni surface-diffusion. The Richardson-Ellingham diagram clearly shows that
Ni has a bad oxide formation ability which could result in a worse corrosion resistance. Indeed, a certain $\mathrm{Ni}-\mathrm{Cr}$ ratio is needed for an ideal corrosion resistance. This is given for AISI 316 [112]. The rising current density before the transpassive area is attributed to Mn surface-diffusion for AISI 310, and Mo surface-diffusion for AISI 316. The breakout potential for sputtered films is dominated by the Fe potential. The carburized AISI 316 sample sputtered at $298 \mathrm{~K}$ cannot be shown due to metallic disbandment, but its characteristics should be similar to those of the sample carburized at $673 \mathrm{~K}$.

As a general observation, the corrosion resistance improves after carburization. More and more $\mathrm{Cr}$ (as well as $\mathrm{Ni}$ ) diffuses to the surface and is used to form a thin oxide layer. This effect is less severe in the case of carburized AISI 316 films. The dimension of the corrosion resistance is given by the metallic disbandment. For inert sputtered AISI 316, we obtained a metallic disbandment at $3.5 \mathrm{~V} ; 7 \mathrm{~V}$ for inert sputtered AISI 316 films sputtered at $673 \mathrm{~K}$ 

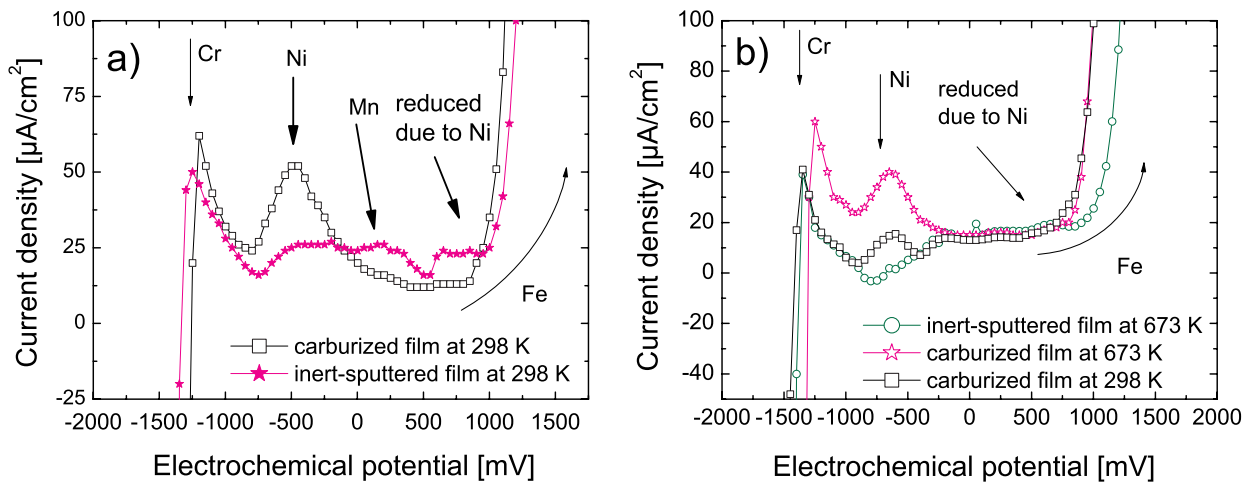

Figure 6.31: Corrosion tests of AISI 310 (a) and AISI 316 (b) sputtered films. All samples were sputtered at a magnetron power of $100 \mathrm{~W}$, inert-sputtered sample with $12 \mathrm{sccm}$ Ar gas flow, carburized samples with $0.01 \mathrm{sccm} \mathrm{CH}_{4}$ gas flow. Temperatures are given in the graphs.

and $8 \mathrm{~V}$ for as-carburized AISI 316 films. Thus, carburization improves corrosion resistance by a factor of 2 in the case of AISI 316 films. As-sputtered AISI 310 films already have a high metallic disbandment $(\sim 8 \mathrm{~V})$. This can be attributed to the high Cr- and Ni-content. For similar carburizing conditions a metallic disbandment at $11 \mathrm{~V}$ for carburized AISI 310 films was obtained. This is an improvement by a factor of nearly 1.5 , which is attributed to the disadvantageous Cr-Ni ratio in AISI 310. The substrate temperature during carburizing has a bigger influence on the corrosion resistance, but it has a marginal influence on the metallic disbandment. Finally, carburizing significantly improves the corrosion resistance.

\subsubsection{Microhardness of carburized stainless steel films}

Nanoindentation was performed to achieve an understanding of the influence of carburization on the microhardness and the mechanical properties. The results are presented in Table 6.8 and ??, where also the carbon content derived from RBS is included.

Different dependencies were observed for the differ-

Table 6.8: Hardness, Young modulus and C-content of carburized AISI 310 films, where $E$ is the elastic modulus and $v$ the poisson ratio

\begin{tabular}{cccc}
\hline $\mathrm{CH}_{4}$ Gas flow & Hardness & $\begin{array}{r}\text { Young modulus } \\
\mathrm{E} /\left(1-\mathrm{v}^{2}\right) \\
{[\mathrm{GPa}]}\end{array}$ & $\begin{array}{c}\text { C-content } \\
{[\mathrm{sccm}]}\end{array}$ \\
\hline 0.10 & $6.1(5)$ & $129(2)$ & 39.75 \\
0.50 & $3.4(2)$ & $98(1)$ & 29.90 \\
0.75 & $4.3(3)$ & $101(1)$ & 52.10 \\
1.00 & $7.3(2)$ & $166(3)$ & 52.90 \\
1.25 & $5.8(3)$ & $120(2)$ & 65.80 \\
\hline
\end{tabular}

Table 6.9: Hardness, Young modulus and C-content of carburized AISI 316 films, where $E$ is the elastic modulus and $v$ the poisson ratio

\begin{tabular}{|c|c|c|c|}
\hline $\begin{array}{c}\mathrm{CH}_{4} \text { Gas flow } \\
{[\mathrm{sccm}]}\end{array}$ & Hardness & 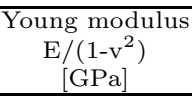 & $\begin{array}{c}\text { C-content } \\
{[a t . \%]}\end{array}$ \\
\hline 0.01 & $5.2(4)$ & $113(1)$ & 60.0 \\
\hline 0.05 & $4.8(1)$ & $105(1)$ & 14.9 \\
\hline 0.10 & $5.1(3)$ & 108(1) & 55.0 \\
\hline 0.50 & $4.1(5)$ & $98(1)$ & 29.9 \\
\hline 0.75 & $5.1(2)$ & 108(1) & 44.9 \\
\hline 1.00 & $4.4(9)$ & $97(1)$ & 59,8 \\
\hline 1.25 & $4.0(1)$ & $88(1)$ & 65.1 \\
\hline
\end{tabular}

ent steel types. While the C-content rises quite linearly in carburized AISI 310 films with the methane gas flow, no clear dependencies are obtained for AISI 316 films. Both phenomena can be explained with the model of Lux and Haubner [113] mentioned in the discussion area. In the case of carburized AISI 310 films, all carbon is used to form carbides and graphite from the vapor phase. For carburized AISI 316 films we assume a higher and faster plasma interaction between $\mathrm{CH}_{x}$ radicals and sputter-adsorbates which can deplete already existing carbides or graphite. The hardness values for the amorphous soft ferromagnetic phases are conspicuous. For AISI 310, this phase exhibits the lowest hardness. Amorphous carburized AISI 316 is is the hardest sample.

\subsubsection{Discussion on a nucleation model for carburized stainless steel films}

Now three questions arise: how can the formation of the amorphous soft ferromagnetic phase be explained and how much carbon is incorporated in the 
films, which finally leads to the question of the carbide formation. According to Lu et al. [13] the first question can be answered as follows: the present Fe-based alloy is associated with the deep eutectic point of the Fe-C system. It is well known that compositions around the deep eutectic point are ideal for glass formation in many systems. As a result, glass formation is greatly favored thermodynamically. Further, the minor addition of Mo could promote glass formation in the Fe-C system by suppressing the formation of the primary phase (i.e. Fe carbides). Because of their limited solubility in $\mathrm{Fe}$ carbides, the molybdenum atoms must redistribute and long-range diffusion is required upon solidification. Thus, the minor addition of Mo could retard the nucleation process.

Another approach is the so-called Carbide Formation Ability (CFA) [114], which evaluates the effect of the composition elements. CFA is based on physicochemical analysis [114] of the carbide formation processes accompanying the primary and secondary crystallization of a weld metal with reliance on quantum-chemical theories that depict the structure of transition metals and carbides. CFA is defined as

$$
\Theta_{i}=\frac{R_{i}}{d_{i}},
$$

where $\mathrm{R}_{i}$ is the radius of the alloy elements and $\mathrm{d}_{i}$ the number of the electrons in d-orbital. The CFAs of common steel elements are given in Table 6.10. The growth mechanism of the fabricated stainless steel films is assumed as a combination of the pre-mentioned models. Whereas the first model prescribes that molybdenum aids a diffusion process which suppresses carbide formation, the CFA model predicts the highest CFA value for molybdenum. The fact that the present carburized steel system consists of five elements, the probability of each atom to interact only with one sort of element is not given. Thus, the CFA model can only be used auxiliary. A clear evidence of this hypothesis could be a topic of further investigations, wherein the ion-distribution functions of carburization processes could be investigated.

In contrast to inert gas sputtered AISI 316 films, the role of $\mathrm{Ni}$ is insignificant. Even the method of instantaneous recording of the electromotive force (MIE) $[93,115]$ was inconclusive. The chemical potential of the films changed from Fe-potential at room temperature to $\mathrm{Fe}_{2} \mathrm{O}_{3}$-potential at $673 \mathrm{~K}$. Thus, the Gibbs-Thomson effect cannot be used to explain the formation of carbides. This backs the thesis of long-range diffusion during nucleation. It can be assumed that - due to the relative abundances of the steel elements - these carbides are $(\mathrm{Fe}, \mathrm{Cr})_{3} \mathrm{C}$ carbides.

In consideration to the present results, an entire conception of the nucleation process which is referred by Lux and Haubner [113] can be stated as follows: during the nucleation process carbonaceous species were adsorbed on the surface. Via vapor phase interaction, e.g. hydrogen recombination and formation of $\mathrm{CH}_{x}$ radicals, free $\mathrm{C}$ atoms were formed. Diffusion processes already inserted by Mo atoms solve the $\mathrm{C}$ atoms in the metal matrix. The carburization initially takes up all available $\mathrm{C}$ out of the vapor phase, until a closed carbide film is formed. With increasing thickness of the carbide film, the $\mathrm{C}$ transport in the metal matrix is slowed down. This leads to an increasing C-content on the surface which induces metastable clustering. The differing carbon content in the carburized stainless steel films is originated in the inserted diffusion process (and thus in the differing carbon take up in the metal matrix) which depends on the processing parameters. If the grain sizes exceeds a critical value, even diamond-like carbon films can be built [113]. This model explains the variety of the observed phases and the different carbon contents in the carburized films. It is reasonable to assume that the solubility limit of $\mathrm{C}$ in $\mathrm{Fe}_{3} \mathrm{C}$ is reached. Excessive C-content exists in form of graphite nanoclusters on top of the film. This is confirmed by Fig. 6.32 which shows the dependence between peak width and carbon content.

A clear tendency can be seen: with higher carbon

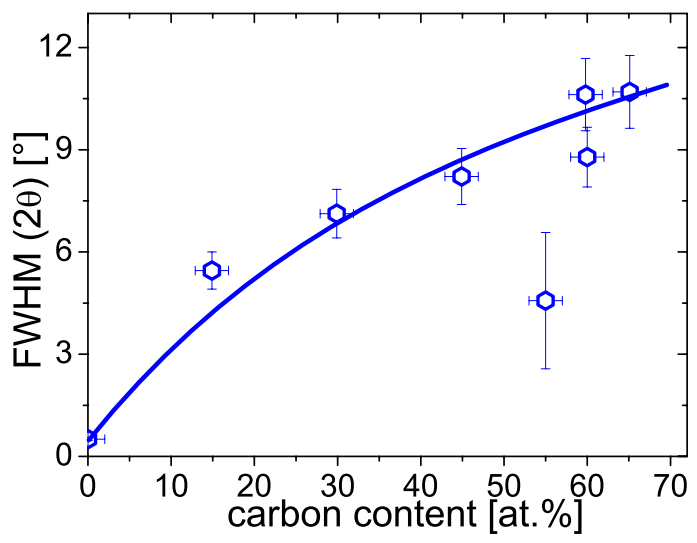

Figure 6.32: Peak width of the first amorphous peak in dependence of the carbon content of reactive sputtered stainless steel films.

concentration, higher peak widths can be reached. Only the sample sputtered at $0.10 \mathrm{sccm} \mathrm{CH}_{4}$ gas flow deviates. The first reflex in the XRD pattern of this sample had to be fitted with two peaks: one attributed to an amorphous phase and one attributed to a crystalline phase. It is reasonable to assume that all XRD pattern could be fitted by two peaks, but there are no clear hints (peak asymmetry etc.) 
Table 6.10: Carbide Formation Ability (CFA) of AISI steels. The lattice constants $a$ are also given.

\begin{tabular}{cccccc}
\hline Element & $\begin{array}{c}\text { Electron } \\
\text { Configuration }\end{array}$ & $\begin{array}{c}\text { Atom radius } \\
{[\AA]}\end{array}$ & $\begin{array}{c}\text { Crystallographic } \\
\text { structure }\end{array}$ & $\begin{array}{c}a \\
{[\AA]}\end{array}$ & CFA \\
\hline $\mathrm{Fe}$ & $3 d^{6} 4 s^{2}$ & 1.260 & $\mathrm{BCC}(\alpha)$ & 2.861 & 0.210 \\
& & & $\mathrm{FCC}(\gamma)$ & 3.564 & \\
\hline $\mathrm{Cr}$ & $3 d^{5} 4 s^{2}$ & 1.270 & $\mathrm{BCC}$ & 2.885 & 0.256 \\
\hline $\mathrm{Ni}$ & $3 d^{8} s^{2}$ & 1.250 & $\mathrm{FCC}$ & 3.520 & 0.156 \\
\hline $\mathrm{Mn}$ & $3 d^{5} 4 s^{2}$ & 1.270 & $\operatorname{SC}(\alpha)$ & 8.894 & 0.254 \\
& & & $\mathrm{SC}(\gamma)$ & 6.300 & \\
& & & $\mathrm{FCC}(\gamma)$ & 3.774 & \\
& & & $\mathrm{BCC}(\delta)$ & 3.720 & \\
\hline $\mathrm{Mo}$ & $4 d^{5} 4 s^{1}$ & 1.390 & $\mathrm{BCC}$ & 2.885 & 0.278 \\
\hline
\end{tabular}

as seen for the $0.10 \mathrm{sccm}$ sample. Even here, no quantitative conclusion can be given how many carbon is solved in the metallic matrix as graphite or as carbide.

\subsubsection{Microstructure of the amor- phous and soft ferromagnetic phase}

The structural nature of the amorphous soft ferromagnetic phase is still unknown, but due to the previous mentioned results, it is reasonable to assume that Mo triggers a diffusion processes which suppresses the formation of carbides and should thus lead to a loss of distal-order and to a dramatic change in the liquidus temperature. With respect to this, TEM, FIM, DSC and EXAFS were carried out to reach a full understanding of the microstructure of amorphous steels.

TEM and FIM analysis As a first attempt to explain the microstructure of the amorphous and soft ferromagnetic phase, TEM patterns were taken, which are shown in Fig. 6.33.

Fig. $6.33 \mathrm{a}$ ) and b) show the dark field patterns of the amorphous and soft ferromagnetic phase. They show nano-crystallites with a diameter of 5-10 nm at the film $/ \mathrm{SiO}_{2}$ interface and the surface of the film. The mostly amorphous structure of the film is confirmed by the selected area diffraction (SAD) pattern, which is depicted in Fig. 6.33 c) and shows the typical behavior of amorphous films. Furthermore, Fig. 6.33 d) shows the high-resolution pattern of the film. Inhomogeneities in metal concentration can be observed. Considering the high carbon concentration ( $\approx 60 \%$ derived by RBS), this decomposition of stainless steel and carbon could be due to diffusion during deposition. This corresponds to our model, that Mo suppresses the primary phase formation and induces long-range diffusion, which finally leads to metastable clustering of graphite as observed in Raman spectroscopy [22].

In order to confirm the TEM results and to get more information on the observed inhomogeneities, a FIM analysis was performed. The result can be readily seen in Fig. 6.34.

The observed FIM pattern predominantly shows

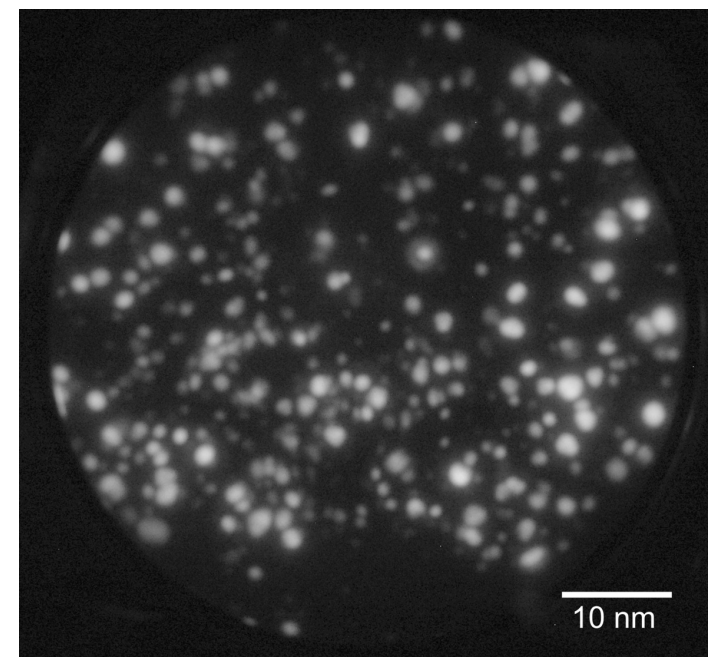

Figure 6.34: FIM pattern of the amorphous and soft ferromagnetic film (sputtered at $298 \mathrm{~K}$ at $0.01 \mathrm{sccm}$ $\left.\mathrm{CH}_{4}\right)$.

the behavior of an amorphous alloy, but there are also appendages of crystallization: in the bottom of Fig. 6.34, the formation of about $10 \mathrm{~nm}$ ringlike atom strings can be observed. According to the literature [116], these characteristics are typical for an initiating crystallization and can be observed for complex intermetallic compounds. Thus, it is reasonable to assume that some of the observed inhomogeneities are nano-crystallites. Summarizing, the amorphous and soft ferromagnetic phase appears as a quasi-metallic glass.

DSC measurement The thermal properties of the amorphous and soft ferromagnetic phase obtained by DSC are shown in Fig. 6.35.

The scan exhibits a glass transition (at $598.8 \mathrm{~K}$ ) and two crystallization events (at 622.05 and $683.45 \mathrm{~K}$ ), indicating that this stainless steel has 


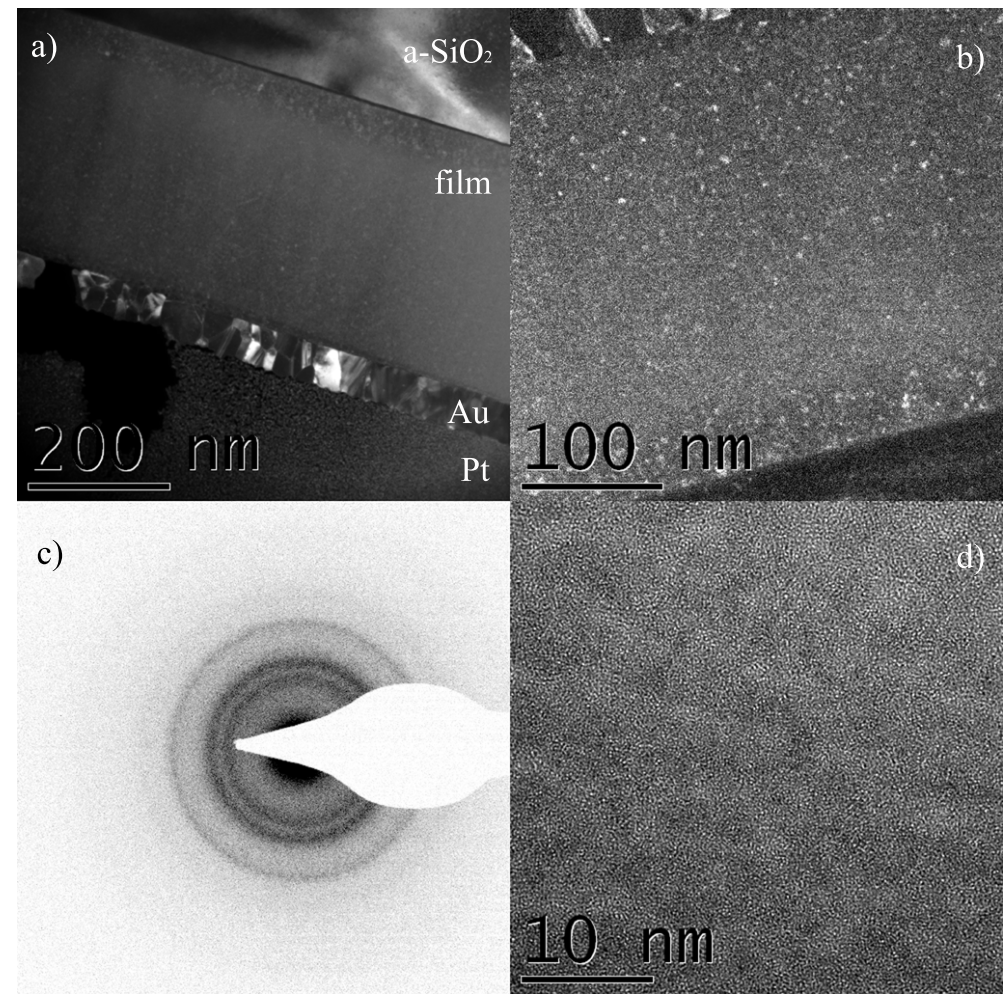

Figure 6.33: TEM pattern of the amorphous and soft ferromagnetic film (sputtered at $298 \mathrm{~K}$ at $0.01 \mathrm{sccm}$ $\mathrm{CH}_{4}$ ). a) and b) dark field pattern, c) SAD pattern and d) HR-TEM of the film.

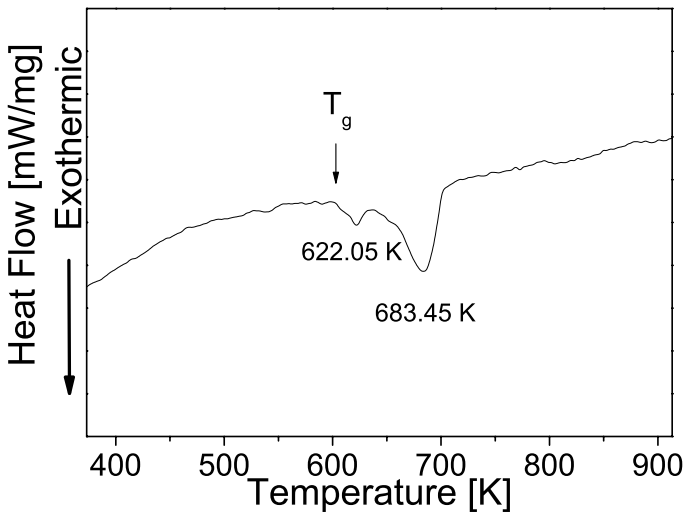

Figure 6.35: DSC curve of the amorphous and soft ferromagnetic film (sputtered at $298 \mathrm{~K}$ at $0.01 \mathrm{sccm}$ $\mathrm{CH}_{4}$ ) measured at a heating rate of $10 \mathrm{~K} / \mathrm{s}$. the arrow refers to the glass transition temperature $\mathrm{T}_{g}$.

a mostly amorphous structure. For AISI 316 stainless steel films we obtained recrystallization at the temperature range 923-1073 K [93], thus the metallic glass behavior is confirmed. The obtained glass transition temperature $\mathrm{T}_{g}$ is significant lower than those given in the literature [12-14], which indicates the potential of the amorphization of conventional steels.
EXAFS analysis of the amorphous and soft ferromagnetic phase To investigate the shortrange order of the amorphous and soft ferromagnetic phase EXAFS experiments were carried out at the ESRF beamline BM29. The investigated depth area was estimated to be $\sim 80 \mathrm{~nm}$ for the Fe k-edge region and $\sim 105 \mathrm{~nm}$ for the Ni k-edge region, respectively, according to the formula reported by Elam et al. [117]. For comparison, EXAFS spectra of $\alpha$-Fe, bulk AISI 316, and $\mathrm{Fe}_{3} \mathrm{C}$ were taken. Data analysis was performed using the HORAE package [65-67] according to following procedure: the origin of the kinetic energy was chosen as the maximum of the first derivative of the absorption edge; the fine-structure oscillations were isolated in the energy space to simulate the continuous atomic cross section; the $\chi(E)$ was converted to $\mathrm{k}$ space and the $k^{2} \chi(k)$ data were Fouriertransformed in the region $2<\mathrm{k}<12 \AA^{-1}$. Figure 6.36 shows the fine-structure oscillations (weighted with factor $k^{2}$ ) of the standards $\alpha-\mathrm{Fe}, \gamma-(\mathrm{Fe}, \mathrm{Cr}, \mathrm{Ni}$ ) (bulk AISI 316), $\mathrm{Fe}_{3} \mathrm{C}$ and the reactive magnetron-sputtered sample deposited with $0.01 \mathrm{sccm} \mathrm{CH}_{4}$ gas flow.

In order to have a quantitative information, the spectra were analyzed by superimposition of theoretical models of different crystal structures calculated by ATOMS and FEFF $[65,67]$ (using a multi- 
Table 6.11: Fit results for the Fe k-edge.

\begin{tabular}{llccccc}
\hline phase & path & $\mathrm{N}_{\text {degen }}$ & $\sigma^{2}$ & $\mathrm{R}_{\text {eff }}(\AA)$ & $\Delta \mathrm{R}(\AA)$ & $\mathrm{R}(\AA)$ \\
\hline$\gamma$-(Fe,Cr,Ni) & $(\mathrm{Fe}, \mathrm{Cr}, \mathrm{Ni})-(\mathrm{Fe}, \mathrm{Cr}, \mathrm{Ni})$ & 12 & 0.013 & 2.579 & -0.148 & 2.430 \\
\hline martensite & $\mathrm{C}-\mathrm{Fe}$ & 2 & 0.109 & 1.484 & 0.405 & 1.889 \\
& $\mathrm{C}-\mathrm{Fe}$ & 4 & 0.109 & 2.026 & 0.405 & 2.431 \\
& $\mathrm{C}-\mathrm{C}$ & 3 & 0.109 & 2.511 & 0.405 & 2.917 \\
\hline (Fe,Cr,Ni)-C & (Fe,Cr,Ni)-C & 3 & 0.019 & 1.426 & -0.142 & 1.284 \\
& (Fe,Cr,Ni)-C & 6 & 0.019 & 2.470 & -0.142 & 2.328 \\
& (Fe,Cr,Ni)-C-C-C & 6 & 0.019 & 2.662 & -0.142 & 2.519 \\
& (Fe,Cr,Ni)-C-C-C & 12 & 0.019 & 2.662 & -0.142 & 2.519 \\
& C-(Fe,Cr,Ni) & 3 & 0.019 & 2.852 & -0.142 & 2.710 \\
& (Fe,Cr,Ni)-C-C-C-C & 3 & 0.019 & 2.853 & -0.142 & 2.711 \\
& (Fe,Cr,Ni)-C-C-C-C & 6 & 0.019 & 2.853 & -0.142 & 2.711 \\
\hline
\end{tabular}

Table 6.12: Fit results for the Ni k-edge.

\begin{tabular}{|c|c|c|c|c|c|c|}
\hline phase & path & $\mathrm{N}_{\text {degen }}$ & $\overline{\sigma^{2}}$ & $\mathrm{R}_{e f f}(\AA)$ & $\Delta \mathrm{R}(\check{\mathrm{A}})$ & $\mathrm{R}(\AA)$ \\
\hline$\gamma-(\mathrm{Fe}, \mathrm{Cr}, \mathrm{Ni})$ & $(\mathrm{Fe}, \mathrm{Cr}, \mathrm{Ni})-(\mathrm{Fe}, \mathrm{Cr}, \mathrm{Ni})$ & 12 & 0.009 & 2.539 & -0.185 & 2.354 \\
\hline \multirow[t]{10}{*}{$\mathrm{Ni}_{3} \mathrm{C}$} & $\mathrm{Ni}-\mathrm{Ni}$ & 2 & 0.008 & 1.517 & -0.124 & 1.394 \\
\hline & $\mathrm{Ni}-\mathrm{C}$ & 2 & 0.008 & 1.564 & -0.124 & 1.440 \\
\hline & $\mathrm{Ni}-\mathrm{Ni}$ & 6 & 0.008 & 1.697 & -0.124 & 1.573 \\
\hline & $\mathrm{Ni}-\mathrm{C}$ & 2 & 0.008 & 1.897 & -0.124 & 1.773 \\
\hline & $\mathrm{Ni}-\mathrm{Ni}-\mathrm{C}-\mathrm{Ni}$ & 8 & 0.008 & 2.413 & -0.124 & 2.289 \\
\hline & $\mathrm{Ni}-\mathrm{C}$ & 2 & 0.008 & 2.429 & -0.124 & 2.305 \\
\hline & $\mathrm{Ni}-\mathrm{Ni}-\mathrm{Ni}-\mathrm{Ni}$ & 12 & 0.008 & 2.456 & -0.124 & 2.332 \\
\hline & $\mathrm{Ni}-\mathrm{C}-\mathrm{Ni}-\mathrm{Ni}$ & 4 & 0.008 & 2.489 & -0.124 & 2.365 \\
\hline & $\mathrm{Ni}-\mathrm{C}-\mathrm{Ni}-\mathrm{Ni}$ & 6 & 0.008 & 2.489 & -0.124 & 2.365 \\
\hline & $\mathrm{Ni}-\mathrm{C}-\mathrm{Ni}-\mathrm{Ni}$ & 8 & 0.008 & 2.579 & -0.124 & 2.455 \\
\hline \multirow[t]{2}{*}{$\alpha-(\mathrm{Fe}, \mathrm{Cr}, \mathrm{Ni})$} & $(\mathrm{Fe}, \mathrm{Cr}, \mathrm{Ni})-(\mathrm{Fe}, \mathrm{Cr}, \mathrm{Ni})$ & 8 & 0.021 & 2.482 & -0.065 & 2.417 \\
\hline & $(\mathrm{Fe}, \mathrm{Cr}, \mathrm{Ni})-(\mathrm{Fe}, \mathrm{Cr}, \mathrm{Ni})$ & 6 & 0.021 & 2.867 & -0.065 & 2.801 \\
\hline
\end{tabular}

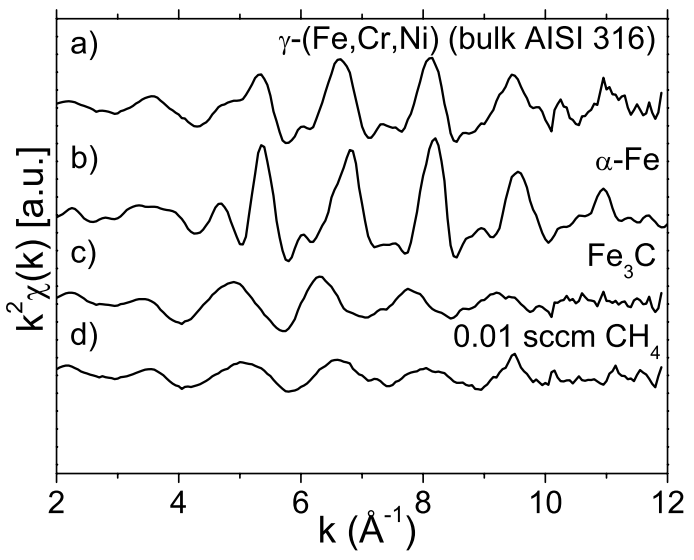

Figure 6.36: Fine-structure oscillations weighted with $k^{2}$ factor of: a) $\gamma$-(Fe, $\mathrm{Cr}, \mathrm{Ni}$ ) (bulk AISI 316), b) $\alpha$-Fe, c) $\mathrm{Fe}_{3} \mathrm{C}$ and the reactive magnetronsputtered sample deposited with $0.01 \mathrm{sccm} \mathrm{CH}_{4}$.

shell model). To minimize the number of variables, $\sigma^{2}$ and $\Delta R$ were set equal for every path of each structure. The back Fourier transform (BFT) was calculated in the region $1.0<\mathrm{R}<2.8 \AA$, which can be seen in Fig. 6.37 recorded at the Fe- and Ni k-edge.

Whereas the BFT modulus of the bulk mate-

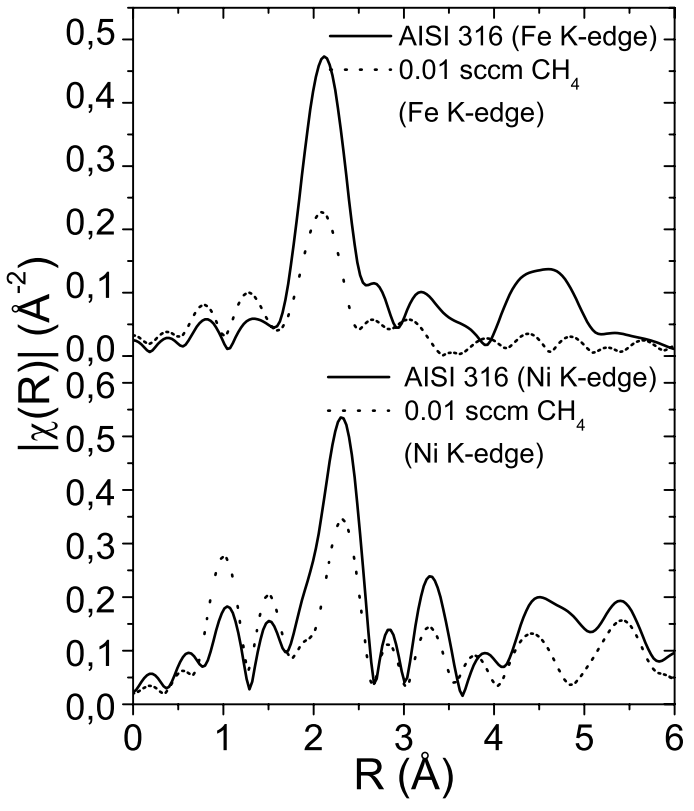

Figure 6.37: BFT moduli of bulk AISI 316 (solid) and the amorphous and soft ferromagnetic film (sputtered at $298 \mathrm{~K}$ at $0.01 \mathrm{sccm} \mathrm{CH}_{4}$ ) (dashed line) measured at the Fe K-edge (top) and at the Ni Kedge (bottom). 
rial can simply be fitted by a face-centered cubic structure, drastic changes in the Fe and Ni environment can be observed for the reactive magnetronsputtered sample. At the Fe k-edge an immense decrease of long-range order can be observed, but the local Fe-environment is dominated by a superimposition of $\gamma-(\mathrm{Fe}, \mathrm{Cr}, \mathrm{Ni})$, martensite and a carbon-rich $(\mathrm{Fe}, \mathrm{Cr}, \mathrm{Ni})$ environment.

At the Ni k-edge the loss of long-range order is not as severe as observed at the Fe k-edge and the BFT can be fitted by a superimposition of $\gamma-(\mathrm{Fe}, \mathrm{Cr}, \mathrm{Ni})$, $\alpha-(\mathrm{Fe}, \mathrm{Cr}, \mathrm{Ni})$ and a trigonal- $\mathrm{Ni}_{3} \mathrm{C}$ phase. The results of the fitting routine can be obtained from Table 6.11 and Table 6.12 (for clarity only the paths in the fitting range are listed).

The reported disorder parameters $\sigma^{2}$ show the poor quality of the crystallinity of the obtained phases, which is in good agreement to TEM, FIM, DSC and as well as to the results mentioned in [22]. According to this analysis, the XRD pattern was revalued, which is presented in Fig. 6.38. Note, all significant reflexes were reproduced by the phases obtained by the EXAFS analysis.

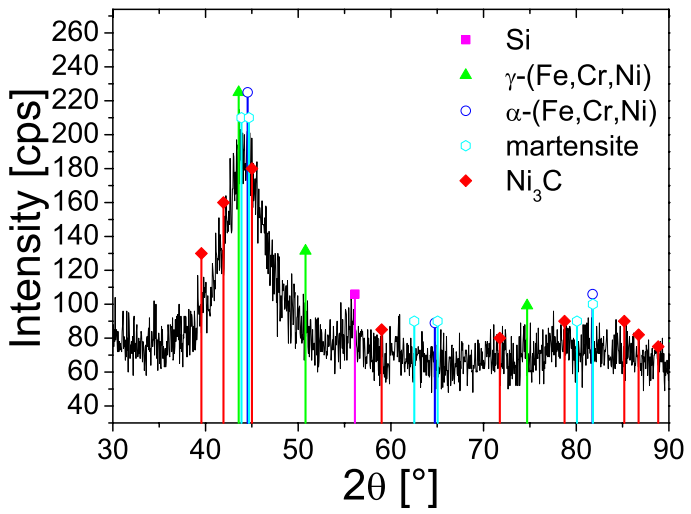

Figure 6.38: XRD pattern of the reactive magnetron-sputtered film deposited at $0.01 \mathrm{sccm}$ $\mathrm{CH}_{4}$ gas flow.

The origin of magnetism In order to explain the origin of ferromagnetism in the reactive magnetron sputtered sample, as shown by CEMS and MOKE, the EXAFS data have to be interpreted. From this analysis two phases are known, which show harder magnetism: martensite and the $\alpha$ $(\mathrm{Fe}, \mathrm{Cr}, \mathrm{Ni})$ phase.

A combination of both phases would result in higher coercitive fields as observed by MOKE. Even in consideration of the high disorder parameter of martensite $\left(\sigma^{2}=0.109\right)$ and of $\alpha-(\mathrm{Fe}, \mathrm{Cr}, \mathrm{Ni})\left(\sigma^{2}=0.021\right)$ obtained by the EXAFS analysis, a resulting coercive field of about 4 Oe would be improbable by a superimposition of these magnetic phases. This leads to the assumption that the carbon diffuses into both structures (as indicated by the high disorder parameters and as indicated by the high-carbon containing ( $\mathrm{Fe}, \mathrm{cr}, \mathrm{Ni}$ )-environment) and decreases the hyperfine field. Indeed, this behavior is derived from Mössbauer analysis for reactive magnetronsputtered stainless steel films [22], which can be seen in Fig. 6.39.

Due to the high carbon concentration and its de-

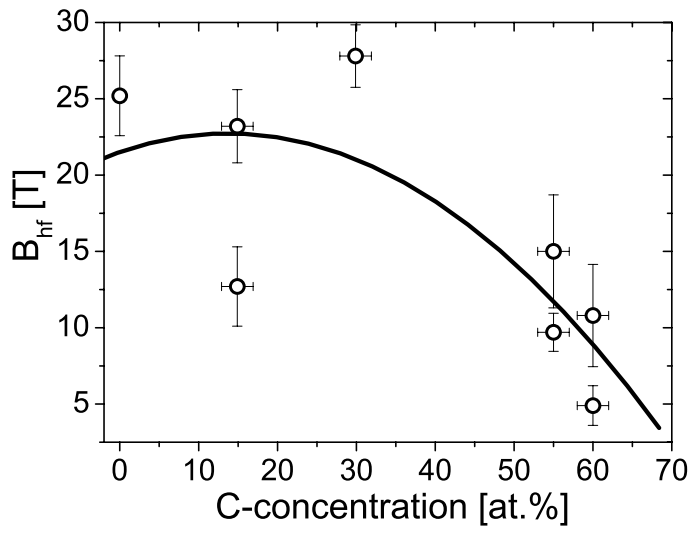

Figure 6.39: Median hyperfine field $\mathrm{B}_{h f}$ in dependence of the carbon content of reactive sputtered stainless steel films.

magnetizing effect, the contribution of both phases to magnetism is adopted as negligible.

Recent studies report on the ferromagnetic behavior of $\mathrm{Ni}_{3} \mathrm{C}[118,119]$, which crystal structure is shown in Fig. 6.40 and was drawn by the crystal visualization software DIAMOND [120].

A linear-muffin-tin-orbital (LMTO) method showed that for ordered $\mathrm{Ni}_{3} \mathrm{C}$ carbon has a s-state character in its lowest valence band, which is populated by strongly hybridized $\mathrm{Ni} \mathrm{p}$-, d- and $\mathrm{C}$ p-states at its bottom [119]. Thus, ordered $\mathrm{Ni}_{3} \mathrm{C}$ is theoretically expected to be non-magnetic.

The origin of the observed magnetism has been ascribed to the presence of crystal defects. According to a LMTO band structure calculation, the $\mathrm{C}$ vacancies which generate locally $\mathrm{Ni}$-rich regions (by removing the hybridization between $\mathrm{Ni}$ and $\mathrm{C}$ states) are believed to sustain the magnetic moments exhibiting spin glassy (SP), paramagnetic (P) and ferromagnetic (FM) properties. The order of magnetism can not be explained by this method, but it additionally predicts the presence of paramagnetic and spin glass phases.

Monte Carlo simulations on the ferromagnetic behavior of amorphous Co-Ni-B and $\mathrm{Fe}-\mathrm{Ni}-\mathrm{B}$ nanoparticles were reported by De Biasi et al. [121,122]. Simulations based on a core-shell model, describ- 


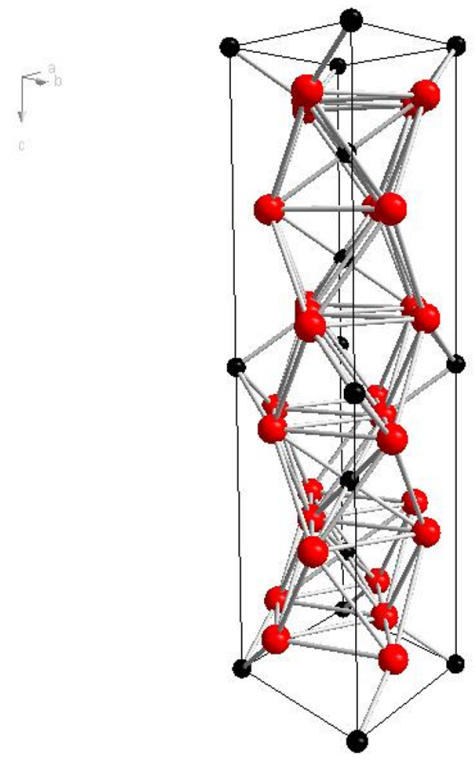

Figure 6.40: Crystal structure of trigonal $\mathrm{Ni}_{3} \mathrm{C}$ phase: space group R -3 c (167), $a=4.5530 \AA$, $c=12.9200 \AA$.

ing the nanoparticles as consisting of a ferromagnetic core and a disordered surface shell, reproduce satisfactorily the different orders of magnetism by randomly oriented spins of the SP surface shell. These spin moments would then contribute to demagnetize the core ferromagnetism via the SP-FM exchange coupling as $H$ approaches zero. Hence, a severely reduced coercivity is observed around $H=0$. He et al. [118] applied this core-shell model to disordered $\mathrm{Ni}_{3} \mathrm{C}$ nanoparticles and were able to explain coercive fields which have the same order as obtained in this work.

Summarizing, ferromagnetism in this amorphous phase can be reduced to a disordered $\mathrm{Ni}_{3} \mathrm{C}$ phase, which is indicated by the disorder parameter (note: good crystallinity is represented for $\sigma<10^{-4}$ ) and is surrounded by a spin glassy surface shell, which demagnetize the ferromagnetic $\mathrm{Ni}_{3} \mathrm{C}$-core.

The influence of the chemical composition To focus on how the differences in chemical composition of conventional steels and steels as described in the literature affect the amorphization, two categories of explanations have to be reconsidered: thermodynamics and kinetics.

As described in $[12,13]$, from a thermodynamical point of view, the superior GFA in BMGs can be associated with a stabilization (through kinetics) outside the equilibrium - the (Fe-C)-system as deep eutectic. This means that the liquid state is energetically favored over the ordered solid state in a large temperature range above the eutectic tem- perature via stabilization of the liquid or through destabilization of distal-order $[32,123]$. Below the eutectic temperature, the force of crystal growth is relatively small. As a consequence, the amorphous state can be reached easier for liquid quenched materials as described in the literature.

For amorphous materials or films which are fabricated by vapor phase techniques as described here, kinetics have to be considered. Also here, we assume that the given system is around the deep eutectic. Atomic-size mismatches induce simultaneous rearrangement of different species of atoms, which suppresses the formation of competing ordered phases and significantly limits the kinetics. As a consequence, glass formation is favored.

Indeed, the enhancement of GFA by atomic-size mismatches and efficient atomic packing is shown by computer simulations [124], topological studies [125] and experimental results [126].

In [125] the local efficiency packing factor $P$ is derived from the ratio $(R)$ of the solute atom radius to the solvent atom radius. Table 6.13 present this ratio of the elements used in this work compared to those mentioned in $[12,13]$.

Smaller values for $R_{i / j}$ and $R_{N}^{*}$ can be ob-

Table 6.13: A list of used alloying elements and selected properties: $\mathrm{r}$ is the atomic radius; $R_{i / j}$ is the the ratio $(R)$ of the solute atom radius to the solvent atom radius; $\mathrm{R}_{N}^{*}$ is the radius ratio, where the subscript $\mathrm{N}$ specifies the particular coordination number; $\sigma^{e^{-}}$is the electronic configuration and CFA the Carbide Formation Ability.

\begin{tabular}{|c|c|c|c|c|c|}
\hline $\mathrm{Z}$ & $\mathrm{r}(\AA)$ & $R_{i / j}$ & $\mathrm{R}_{N}^{*}$ & $\sigma^{e}$ & CFA \\
\hline & & $\mathrm{Ni}$ & & & \\
\hline $\mathrm{Fe}$ & 1.26 & 1.016 & $\mathrm{R}_{13}^{*}$ & {$[\mathrm{Ar}] 3 \mathrm{~d}^{6} 4 \mathrm{~s}^{1}$} & 0.210 \\
\hline $\mathrm{Cr}$ & 1.27 & 1.024 & $\mathrm{R}_{13}^{*}$ & {$[\mathrm{Ar}] 3 \mathrm{~d}^{5} 4 \mathrm{~s}^{1}$} & 0.256 \\
\hline $\mathrm{Ni}$ & 1.24 & - & - & {$[\mathrm{Ar}] 3 \mathrm{~d}^{6} 4 \mathrm{~s}^{2}$} & 0.156 \\
\hline Mo & 1.39 & $\begin{array}{c}1.120 \\
\mathrm{Fe}\end{array}$ & $\mathrm{R}_{15}^{*}$ & {$[\mathrm{Kr}] 4 \mathrm{~d}^{5} 5 \mathrm{~s}^{1}$} & 0.278 \\
\hline \multirow[t]{2}{*}{$\mathrm{C}$} & 0.77 & 1.636 & $\mathrm{R}_{23}^{*}$ & {$[\mathrm{He}] 2 \mathrm{~s}^{2} 2 \mathrm{p}^{2}$} & - \\
\hline & & $\overline{\mathrm{Cu}}$ & & & \\
\hline $\mathrm{Cu}$ & 1.27 & - & - & {$[\mathrm{Ar}] 3 \mathrm{~d}^{10} 4 \mathrm{~s}^{1}$} & 0.127 \\
\hline $\mathrm{Zr}$ & 1.59 & 1.244 & $\mathrm{R}_{17}^{*}$ & {$[\mathrm{Kr}] 4 \mathrm{~d}^{2} 5 \mathrm{~s}^{2}$} & 0.795 \\
\hline $\mathrm{Al}$ & 1.43 & 1.126 & $\mathrm{R}_{15}^{*}$ & {$[\mathrm{Ne}] 3 \mathrm{~s}^{2} 3 \mathrm{p}^{1}$} & - \\
\hline $\mathrm{Y}$ & 1.80 & 1.370 & $\mathrm{R}_{19}^{*}$ & {$[\mathrm{Kr}] 4 \mathrm{~d}^{1} 5 \mathrm{~s}^{2}$} & 1.800 \\
\hline & & Co & & & \\
\hline $\mathrm{Fe}$ & 1.26 & 1.008 & $\mathrm{R}_{13}^{*}$ & {$[\mathrm{Ar}] 3 \mathrm{~d}^{6} 4 \mathrm{~s}^{1}$} & 0.210 \\
\hline $\mathrm{Cr}$ & 1.27 & 1.016 & $\mathrm{R}_{13}^{*}$ & {$[\mathrm{Ar}] 3 \mathrm{~d}^{5} 4 \mathrm{~s}^{1}$} & 0.256 \\
\hline Co & 1.25 & - & 10 & {$[\mathrm{Ar}] 3 \mathrm{~d}^{7} 4 \mathrm{~s}^{2}$} & 0.179 \\
\hline Mo & 1.39 & 1.112 & $\mathrm{R}_{14}^{*}$ & {$[\mathrm{Kr}] 4 \mathrm{~d}^{5} 5 \mathrm{~s}^{1}$} & 0.278 \\
\hline $\mathrm{Mn}$ & 1.26 & 1.008 & $\mathrm{R}_{13}^{*}$ & {$[\operatorname{Ar}] 4 d^{5} 4 s^{2}$} & 0.252 \\
\hline $\mathrm{Y}$ & 1.80 & $\begin{array}{c}1.440 \\
\mathrm{Fe}\end{array}$ & $\mathrm{R}_{19}^{*}$ & {$[\mathrm{Kr}] 4 \mathrm{~d}^{1} 5 \mathrm{~s}^{2}$} & 1.800 \\
\hline $\mathrm{C}$ & 0.77 & 1.636 & $\mathrm{R}_{2}^{*}$ & {$[\mathrm{He}] 2 \mathrm{~s}^{2} 2 \mathrm{p}^{2}$} & 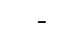 \\
\hline B & 0.98 & 1.286 & $\mathrm{R}_{17}^{*}$ & {$[\mathrm{He}] 2 \mathrm{~s}^{2} 2 \mathrm{p}^{1}$} & - \\
\hline
\end{tabular}

tained for this present work compared to those of the literature, which is originated in the similarity of radii of the alloying elements used in this 
study. According to [125], an efficiency packing factor $P \approx 0.76-0.77$ is derived by taking the ratio of the sum of all atomic volumes to the total volume concerned, which is slightly higher than that derived in [12]. This is even larger than the 0.74 of fcc-crystals, which confirms that our used system is highly efficiently packed after carburization. As a first summary we can stress out, that the amorphization of conventional steels is more complicated than the amorphization of Zr-based steels. This is confirmed by the low coordination number $N$, which indicates a lower atomic-mismatch interaction and thus, a shorter solidification time.

This is confirmed by the negative heat of mixing $\Delta H^{m i x}$, which is also reported to benefit GFA. According to [127], $\Delta H^{\text {mix }}$ for $\mathrm{Fe}-\mathrm{Cr}, \mathrm{Cr}-\mathrm{Ni}$ and $\mathrm{Fe}-\mathrm{Ni}$ are $-4,-4$ and $-5 \mathrm{~kJ} \cdot \mathrm{mol}^{-1}$, and $-1 \mathrm{~kJ} \cdot \mathrm{mol}^{-1}$ for the ternary Fe-Cr-Ni system. According to [12], an improvement of local packing efficiency and repression of long-range diffusion of atoms can be obtained by negative $\Delta H^{\text {mix }}$ by enhancing interactions among components, which promote chemical short-range order. The $\Delta H^{m i x}$ for the steel used in [12] is $4-6$ times higher, which is consistent with the observed coordination numbers.

Now the question arise, in which way conventional AISI 316 can be driven to the amorphous state.

Already during inert sputtering we assume a noninsignificant part of kinetic participation, wherein the $\mathrm{Ni}$ content is decreased under the critical $\gamma$ stabilizing content and finally leads to ferromagnetic stainless steel films [93]. Also here, the Mo triggers a long-range diffusion, which then could lead to a modification of the sticking coefficient, which is inversely proportional to the exponential of the temperature and also inversely proportional to the square of the distance between atomic steps (and thus indirectly proportional to the atomic radius) on the surface [128]. The diminution of $\mathrm{Ni}$ content can be observed in several types of steels $[93,129]$.

The introduction of $\mathrm{C}$ during carburization additionally disturbs the nucleation process by establishing higher probabilities of coordination as represented by $\mathrm{R}_{23}^{*}$. This leads to simultaneous rearrangement of different species of atoms, which suppresses the formation of competing ordered phases. As a consequence, phases outside the equilibrium can be formed like the disordered trigonal $\mathrm{Ni}_{3} \mathrm{C}$ phase. This is confirmed by the Carbide Formation Ability (CFA) [22, 114], which were derived from the electronic configuration $\sigma^{e^{-}}$and were given in Table 6.13.

$\mathrm{Ni}$ has the lowest CFA of all elements, nevertheless, the observed $\mathrm{Ni}_{3} \mathrm{C}$ carbide is formed instead of the established carbides (i.e. $\mathrm{Fe}_{3} \mathrm{C}$ etc.).
The main difference between the steel used in this study and that used in [13] is the number of alloying elements. Here, a quintuple system is used, whereas $\mathrm{Lu}$ et al. chose a octuple system to enhance the coordination number and thus the interatomic interaction (which is supported by the borocarburization of that system).

The next step should be the amorphization of conventional steels via liquid quenching. However, first experiments showed severe problems due to the formation of Cr-oxides. Upcoming experiments should deal with the introduction of various carrier gases which should prevent the oxide formation in such a way that thermodynamics and kinetics were not hardly dominated by competing processes. 


\subsection{Results for nitrided stain- less steel films}

The films were sputter-deposited with an rf magnetron onto amorphous $\mathrm{SiO}_{2}$ substrates (oxidized $\mathrm{Si}(100)$ wafer of $0.5 \mathrm{~mm}$ thickness, pre-cleaned with acetone and oxidized in air, no further treatment) utilizing a commercial AISI 316 (X5CrNiMo17-12$2,1.4401)$ sputter-target. The target-substrate distance was set to $10 \mathrm{~cm}$. The processing parameters were always $100 \mathrm{~W}$ magnetron power at a constant total gas flow of $12 \mathrm{sccm}$ during deposition. Several sample series were deposited at different $\mathrm{N}_{2}$ flows $(0.00-1.00 \mathrm{sccm})$. The sputter rate was always in the range of $0.1-0.2 \mathrm{~nm} / \mathrm{s}$ and was depending on the gas flow parameters. The deposition procedure were carried out in the same way as for as-carburized stainless steel films. The deposition parameters and the resulting thicknesses and growth rates are summarized in Table 6.14. From

Table 6.14: Gas flow $j$, deposition time $t$, real film thickness $d$ (as measured by Rutherford Backscattering Spectrometry (RBS) after deposition) and derived growth rate $g=\frac{d}{t}$ for the deposited nitrided films. All samples were deposited at $298 \mathrm{~K}$ with a magnetron power of $100 \mathrm{~W}$ and a target-substrate

\begin{tabular}{cccccr} 
distance of $10 \mathrm{~cm}$. & & & \\
\hline sample & $\begin{array}{c}j_{\mathrm{N}_{2}} \\
{[\mathrm{sccm}]}\end{array}$ & $\begin{array}{c}j \mathrm{Ar} \\
{[\mathrm{sccm}]}\end{array}$ & $\begin{array}{c}t \\
{[\mathrm{~min}]}\end{array}$ & $\begin{array}{c}d \\
{[\mathrm{~nm}]}\end{array}$ & {$[\mathrm{nm} / \mathrm{min}]$} \\
\hline N0 & 0.00 & 12.00 & $17: 00$ & $205(10)$ & $12.06(92)$ \\
N1 & 0.01 & 11.99 & $16: 43$ & $272(10)$ & $16.27(121)$ \\
N5 & 0.05 & 11.95 & $18: 22$ & $274(10)$ & $14.92(100)$ \\
N10 & 0.10 & 11.90 & $19: 09$ & $256(10)$ & $13.37(88)$ \\
N50 & 0.50 & 11.50 & $24: 15$ & $371(10)$ & $15.30(76)$ \\
N75 & 0.75 & 11.25 & $26: 40$ & $488(10)$ & $18.30(81)$ \\
N100 & 1.00 & 11.00 & $27: 52$ & $468(10)$ & $16.79(73)$ \\
\hline
\end{tabular}

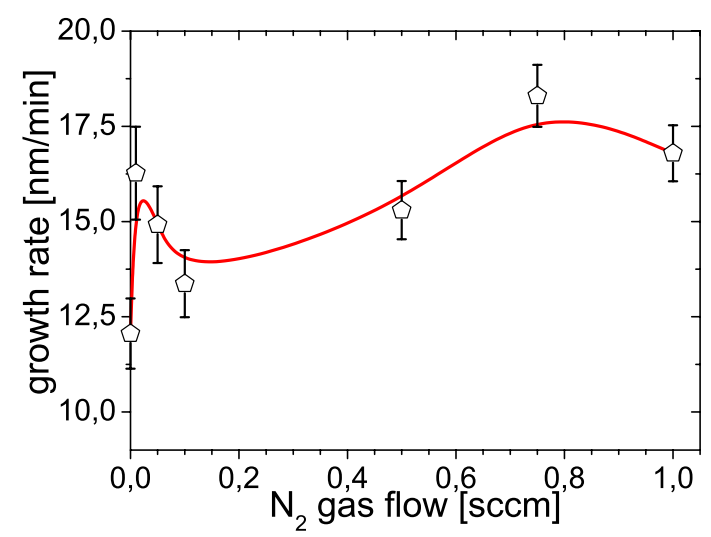

Figure 6.41: Growth rate $g$ as a function of the $\mathrm{N}_{2}$ gas flow $j_{\mathrm{N}_{2}}$ for the room temperature deposition (line is only to guide the eye).

these data it is derived that the growth rate has a sinusoidal form (Fig. 6.41) with its first maximum at $0.03 \mathrm{sccm}$ and its second maximum at $0.80 \mathrm{sccm}$ $\mathrm{N}_{2}$ gas flow, which is contrary to carburized samples, where growth rate drops more or less exponentially from about $12 \mathrm{~nm} / \mathrm{min}$ for the inert sputtering to half of this value for higher methane flows [22]. As be seen below, these parameters represent two metastable phases: an amorphous and soft ferromagnetic phase rich in nitrogen (@ $0.03 \mathrm{sccm} \mathrm{N}_{2}$ gas flow) and the cubic (Fe,Cr, Ni)N phase (@ $0.80 \mathrm{sccm}$ $\mathrm{N}_{2}$ gas flow).

\subsubsection{Deposition at room tempera- ture and influence of the nitro- gen gas flow}

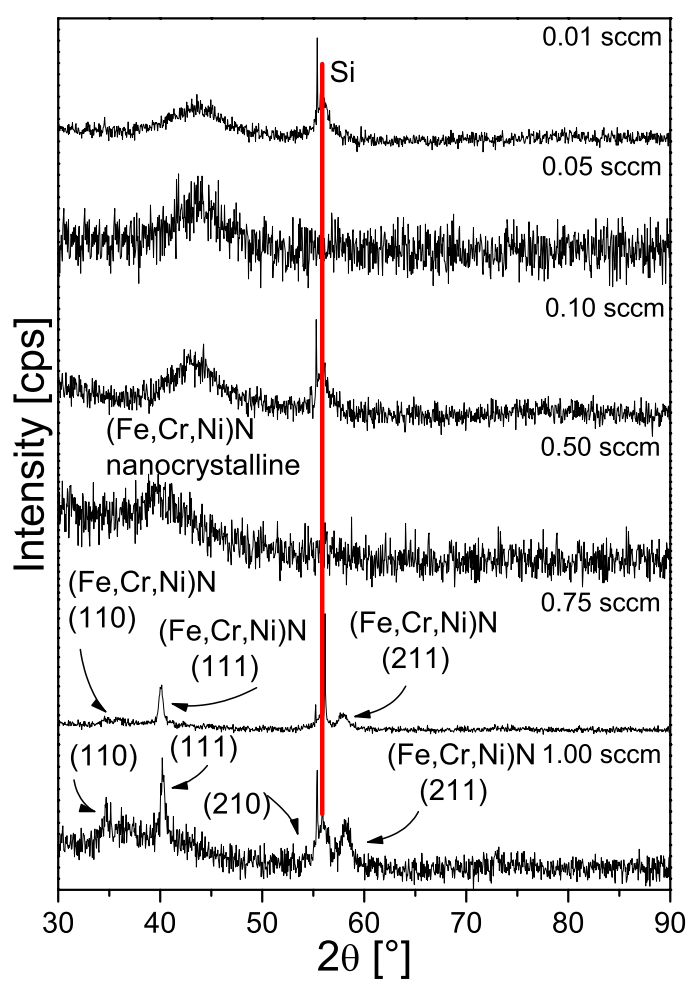

Figure 6.42: GIXRD $\left(2^{\circ}\right)$ spectra of the nitrided films. The $\mathrm{N}_{2}$ gas flows are given in the graph; the peaks of the $\mathrm{ZnS}$-type $(\mathrm{Fe}, \mathrm{Cr}, \mathrm{Ni}) \mathrm{N}$ phase are indexed.

The nitrogen gas flow was systematically increased from $0.01 \mathrm{sccm}$ to $1.00 \mathrm{sccm}$. For the most deposited films, the reactive magnetron deposited and nitrided samples show the typical broad XRD appearance of amorphous materials, as seen in Fig. 6.42. Only exceptions are the nitrided samples deposited with an nitrogen gas flow of 0.75 and 1.00 sccm. According to [130], these reflections can be attributed to the ZnS-type 
Table 6.15: Mössbauer fitting results of the nitrided AISI 316 samples, deposited at room temperature with a magnetron power of $100 \mathrm{~W}(f$ - area fraction (error), mean values of $\langle\delta\rangle$ - isomer shift, $\langle\Delta\rangle-$ the quadrupole splitting for the paramagnetic subspectra, $\langle\epsilon\rangle$ the quadrupole splitting for the magnetic subspectra, $B$ - hyperfine field).

\begin{tabular}{ccrrrrrr}
\hline $\begin{array}{c}\mathrm{N}_{2} \\
{[\mathrm{sccm}]}\end{array}$ & part & $\begin{array}{r}f \\
{[\%]}\end{array}$ & $\begin{array}{r}\langle\delta\rangle \\
{[\mathrm{mm} / \mathrm{s}]}\end{array}$ & $\begin{array}{r}\langle\Delta\rangle,\langle\epsilon\rangle \\
{[\mathrm{mm} / \mathrm{s}]}\end{array}$ & $\begin{array}{r}\sigma_{\Delta} \\
{[\mathrm{mm} / \mathrm{s}]}\end{array}$ & $\begin{array}{r}\langle B\rangle \\
{[\mathrm{T}]}\end{array}$ & $\begin{array}{r}\sigma_{B} \\
{[\mathrm{~T}]}\end{array}$ \\
\hline 0.01 & ferro & $100.0(1)$ & -0.02 & 0.05 & & $23.4(3)$ & $6.5(3)$ \\
\hline 0.05 & ferro-1 & $95.5(15)$ & 0.01 & -0.02 & & $18.2(34)$ & $9.8(3)$ \\
& ferro-h & $2.3(24)$ & 0.01 & -0.02 & & $40.7(16)$ & $3.8(21)$ \\
& para & $2.2(2)$ & 0.28 & 0.58 & & & \\
\hline 0.10 & ferro-1 & $42.0(10)$ & 0.10 & -0.01 & & $20.3(14)$ & $4.2(59)$ \\
& ferro-h & $58.0(13)$ & 0.10 & -0.01 & & $24.0(49)$ & $7.9(35)$ \\
\hline 0.50 & para-1 & $61.2(19)$ & 0.28 & 0.45 & 0.36 & & \\
& para-2 & $38.8(44)$ & & 1.02 & 0.36 & & \\
\hline 0.75 & para & $100.0(1)$ & 0.10 & 0.55 & 0.48 & & \\
\hline 1.00 & para-1 & $69.0(13)$ & 0.29 & 0.48 & 0.28 & & \\
& para-2 & $31.0(28)$ & 0.17 & 0.54 & 0.28 & & \\
\hline
\end{tabular}

$\gamma^{\prime \prime}-(\mathrm{Fe}, \mathrm{Cr}, \mathrm{Ni}) \mathrm{N}$ phase, which exhibits a lattice constant of $a=0.392(22) \mathrm{nm}$.

Fig. 6.43 shows the position and the width of the first broad peak for the spectra shown in Fig. 6.42. There is a clear tendency for peak position and peak width, which both decrease with increasing $\mathrm{N}_{2}$ flow, having its minimum at $0.75 \mathrm{sccm} \mathrm{N}_{2}$ gas flow, before the peak width and especially the peak position rises again.

As a first conclusion a phase transition from the

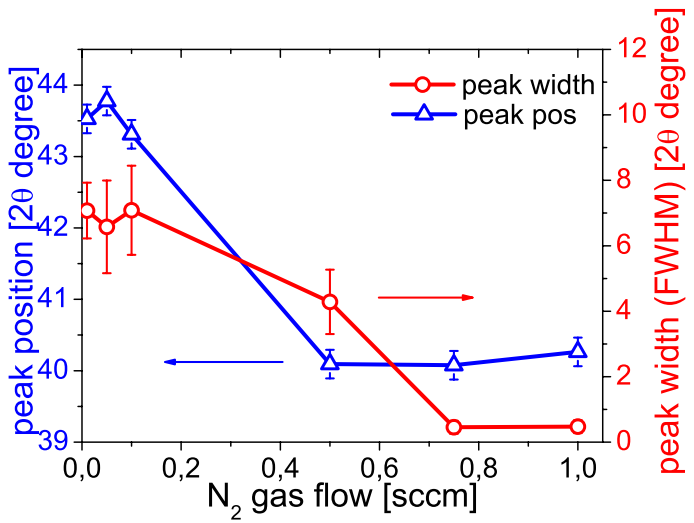

Figure 6.43: Peak position and peak width of the first broad peak in the XRD spectra of the reactive sputtered stainless steel films.

formation of an amorphous to a cubic (Fe,Cr,Ni)N phase can stress out. The earlier phase is formed at a $\mathrm{N}_{2}$ gas flow of $0.75 \mathrm{sccm}$ (with a lattice parameter of $a=0.392(22) \mathrm{nm})$. Further increasing of the $\mathrm{N}_{2}$ gas flow to $1.00 \mathrm{sccm} \mathrm{N}_{2}$ gas flow, the XRD pattern of the nitrided sample $(a=0.394(33) \mathrm{nm})$ shows a decrease of the grain size. This implies, that with higher gas flow the peak width decreases and thus the grain size rises. This tendency is also confirmed by Fig. 6.41 and Fig. 6.43. The discrepancy between the lattice parameters found in this study and in the literature [130] $(a=0.389 \mathrm{~nm})$ may be caused by the presence of Chromium, Nickel and Molybdenum which is known to enlarge the lattice. Fig. 6.44 summarizes the CEMS measurements of the samples with increasing gas flow. They show the typical Mössbauer spectra of amorphous materials. Spectra in a-c are magnetically split, those in $\mathrm{d}$ )-f) are non-magnetic. This observation is consistent with the XRD results in Fig. 6.42. The fitted hyperfine parameters are presented in Table 6.15.

For these spectra, hyperfine field and quadrupole splitting distributions were assumed, respectively. These distributions are attributed to an amorphous stainless steel nitrogen-alloy, possibly rich in nitrogen (as also seen for carburized samples). The quadrupole splitting distributions are broad and close to the values of the cubic nitride. Indeed a single line analysis shows that the observed spectra can be identified as the $\gamma^{\prime \prime}$-FeN phase. The results of the single line analysis are given in Table 6.16 .

Summarizing, all samples below $0.50 \mathrm{sccm} \mathrm{N}_{2}$ gas flow appear as an amorphous material which shows magnetic behavior.

These Mössbauer results were confirmed by MOKE measurements. Figure 6.45 shows the result of the MOKE measurements for two samples: nitrided with $0.01 \mathrm{sccm} \mathrm{N}_{2}$ or $0.10 \mathrm{sccm} \mathrm{N}_{2}$ gas flow. Both result in identical MOKE spectra. 

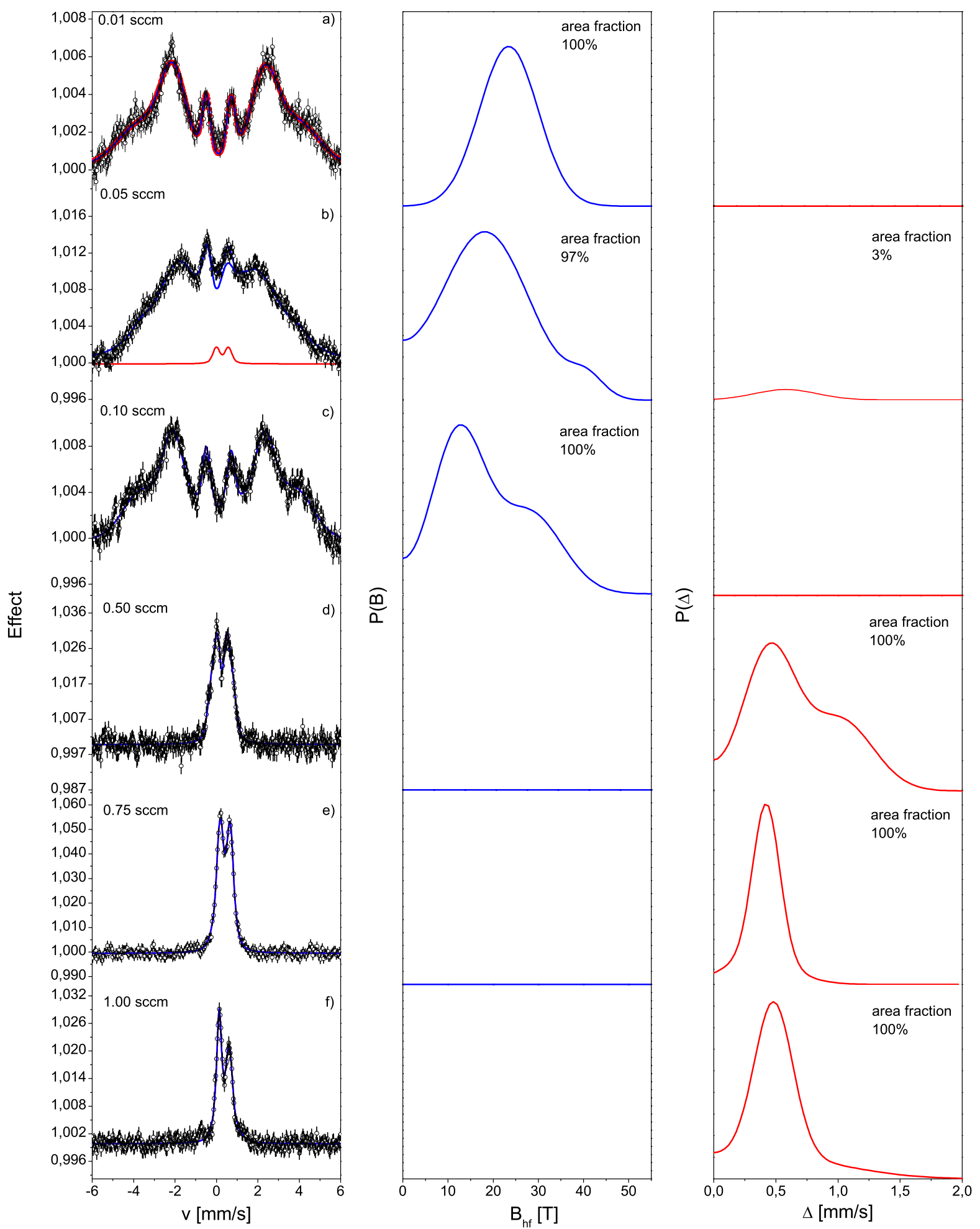

Figure 6.44: Mössbauer spectra of the reactive sputtered AISI 316 films. The numbers in the graphs represent the nitrogen gas flow. 
Table 6.16: Hyperfine interaction parameters of the singe line analysis of the as-sputtered samples shown in Fig. 6.44 e) and f). Hyperfine parameters: $\delta$ is the isomer shift, QS the quadrupole splitting, $\Gamma$ the line width (HWHM), and RA the relative area.

\begin{tabular}{ccccc}
\hline $\begin{array}{c}\mathrm{N}_{2} \\
{[\mathrm{sccm}]}\end{array}$ & $\begin{array}{c}\delta \\
{[\mathrm{mm} / \mathrm{s}]}\end{array}$ & $\begin{array}{c}\mathrm{QS} \\
{[\mathrm{mm} / \mathrm{s}]}\end{array}$ & $\begin{array}{c}\Gamma \\
{[\mathrm{mm} / \mathrm{s}]}\end{array}$ & $\begin{array}{c}\mathrm{RA} \\
{[\%]}\end{array}$ \\
\hline 0.75 & $0.10(3)$ & & 0.20 & $16.6(13)$ \\
& $0.48(4)$ & & 0.20 & $6.3(21)$ \\
& $0.45(1)$ & $0.46(2)$ & 0.20 & $77.1(21)$ \\
\hline 1.00 & $0.09(5)$ & & 0.20 & $18.2(47)$ \\
& $0.48(9)$ & & 0.20 & $4.6(56)$ \\
& $0.39(2)$ & $0.48(2)$ & 0.20 & $77.2(45)$ \\
\hline
\end{tabular}
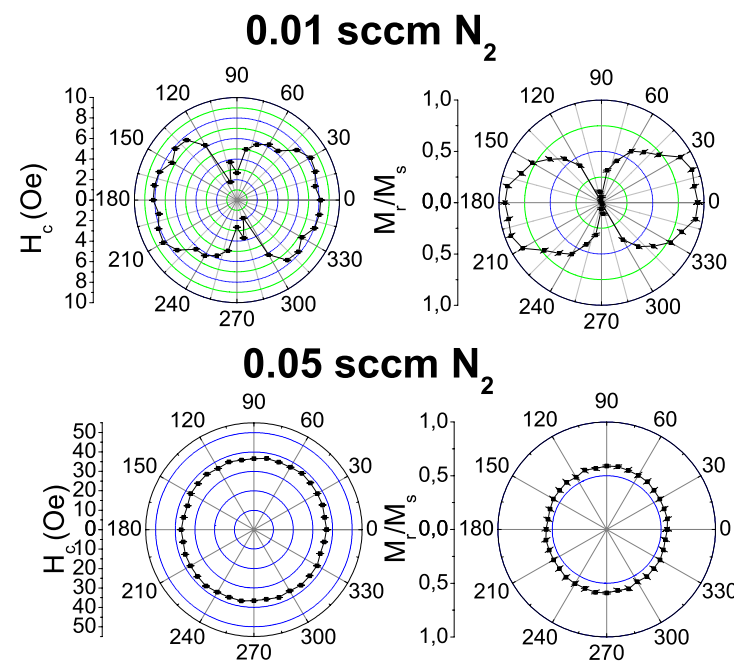

Figure 6.45: Angular scans of the reactive sputtered films with: (top) $0.01 \mathrm{sccm} \mathrm{N}_{2}$ and b) (bottom) $0.05 \mathrm{sccm} \mathrm{N}_{2}$ gas flow. The polar diagrams of the coercive field $H_{\mathrm{C}}$ are shown left and the relative remanence $M_{\mathrm{R}} / M_{\mathrm{S}}$ at the right.

A uniaxial anisotropy of about $75 \%$ can be seen with the maximum of the remanence around $180^{\circ}$. This behavior could be due to magnetostriction effects and stresses in the film. The coercive field $H_{C}$ of both samples is approximately 4 Oe. Figure $6.45 \mathrm{~b}$ ) shows the MOKE result of the as-nitrided sample sputtered with $0.05 \mathrm{sccm} \mathrm{N}_{2}$ gas flow. It does not exhibit any anisotropy. The coercive field was derived as 30 Oe. The samples with CEMS spectra shown in Fig. 6.44d-f) do not show any magnetic behavior (e.g. no hysteresis loops in MOKE), which is in good agreement. These observations have now to be correlated to the nitrogen content of the films.

The EDX analyses of the deposited films revealed the original composition of the sputtering targets within the experimental limits. Unfortunately, the EDX did not allow to accurately determine elements lighter than oxygen. Therefore, in order to evaluate the amount of incorporated nitrogen, RBS measurements were carried out, whose results are shown in Fig. 6.46.

The thickness of the films as obtained from the RBS analysis was already given in Table 6.14. The nitrogen concentration of the films as obtained from the RBS analysis is given in Fig. 6.47 and can reach almost $55(2)$ at.\%. This seems to be a clear increasing correlation of the nitrogen content with the $\mathrm{N}_{2}$ gas flow, with a minimum at $17 \%$ nitrogen for $0.05 \mathrm{sccm} \mathrm{N} \mathrm{N}_{2}$ flow. At $1.00 \mathrm{sccm} \mathrm{N} \mathrm{N}_{2}$ flow the nitrogen content decreases again; this is in good agreement with all previously mentioned results.

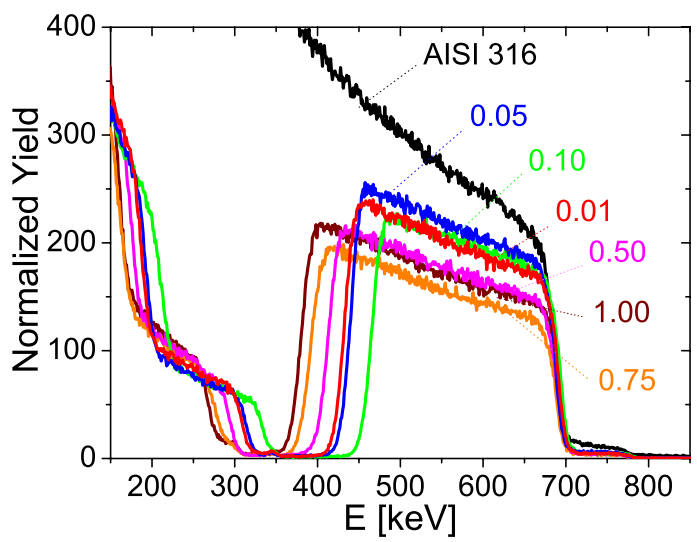

Figure 6.46: RBS spectra of the reactively sputtered films. The $\mathrm{N}_{2}$ flow is given in the graph.

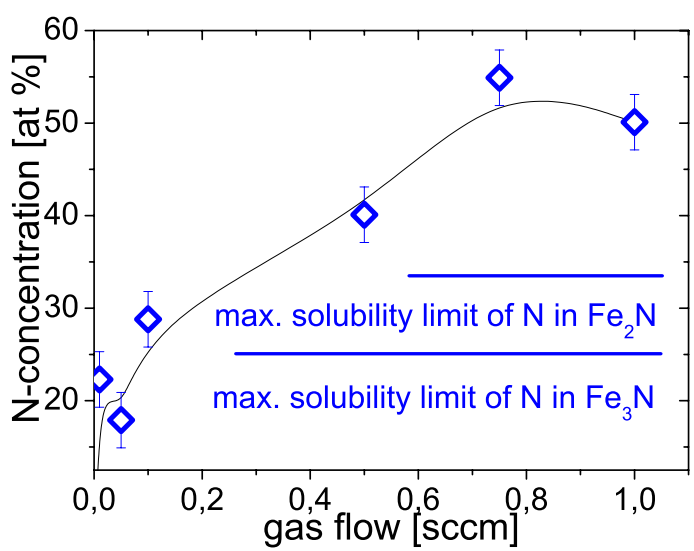

Figure 6.47: Nitrogen content in the reactively sputtered films as derived from the RBS analysis versus the $\mathrm{N}_{2}$ gas flow.

To confirm the observed nitrogen contents, RNRA depth-profiling measurements were performed, which are shown in Fig. 6.48. The RNRA analysis shows the same tendency as the RBS measurements, but the nitrogen contents derived from 


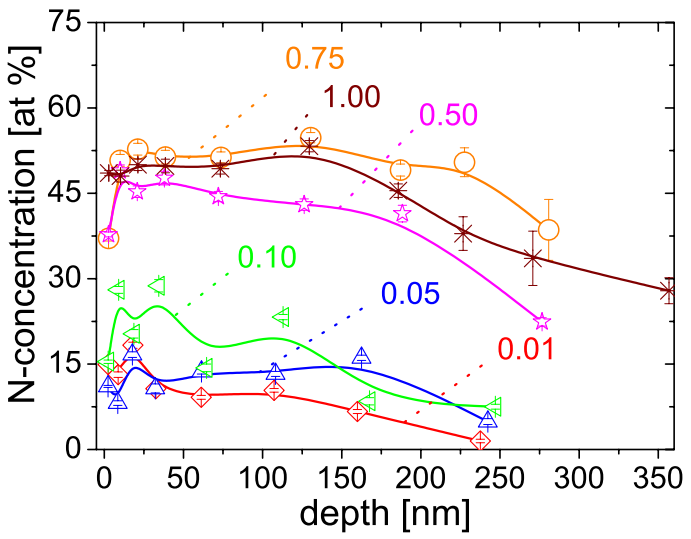

Figure 6.48: RNRA depth-profiling results of the as-nitrided samples. The $\mathrm{N}_{2}$ gas flow are given in the graph.

RNRA are slightly smaller than the RBS results (normally 3 at.\%). Thus, the maximum nitrogen content derived from this data is $52(2)$ at. $\%$. We then can conclude, that the $(\mathrm{Fe}, \mathrm{Cr}, \mathrm{Ni}) \mathrm{N}$ phase is formed at $0.75 \mathrm{sccm} \mathrm{N}_{2}$ gas flow.

\subsubsection{PAC characterization of the vacuum annealed soft ferro- magnetic phase}

To study the influence of vacuum annealing of the amorphous and soft ferromagnetic phase found at $0.05 \mathrm{sccm} \mathrm{N} \mathrm{N}_{2},{ }^{111} \mathrm{In}$ atoms were implanted at an energy of $400 \mathrm{keV}$ at the $530 \mathrm{kV}$ IONAS accelerator facility in Göttingen and then carefully analyzed by PAC at 10 and $298 \mathrm{~K}$. After anlayzing the as-nitrided sample, it was vacuum annealed $\left(p_{a}=10^{-3} \mathrm{~Pa}\right)$ at $973 \mathrm{~K}$ for 2 hours. The results of the PAC measurement are shown in Fig. 6.49. The spectra measured at $10 \mathrm{~K}$ did not show any changes in comparison to the as-nitrided sample recorded at $298 \mathrm{~K}$. Therefore, only the spectra recorded at $298 \mathrm{~K}$ for the as-nitrided and vacuum annealed sample are shown in Fig. 6.49. As the PAC spectra give no indication of a magnetic hyperfine field, they have been fitted assuming an EFG-distribution.

Fig. 6.49 a) shows the typical behavior of amorphous materials, where the PAC probe has no defined position in the matrix due to the absence of crystalline order. As a consequence, the quadrupole interaction frequency $\nu_{Q}$ shows a broad frequency distribution caused by randomly excited EFGs (note: this system consists of five elements and thus, there are 14 combinations of pair interactions). No further information can be taken from
Table 6.17: PAC interaction parameters of the annealed amorphous and soft ferromagnetic phase at $973 \mathrm{~K}$ for 2 hours shown in Fig. $6.49 \mathrm{~b}$ ). Hyperfine parameters: $\nu_{Q}$ is the quadrupole interaction frequency, $\delta$ the damping of $\nu_{Q}, \eta$ the asymmetry parameter, and RA the relative area.

\begin{tabular}{cccc}
\hline$\nu_{Q}$ & $\delta$ & $\eta$ & $\begin{array}{l}\text { RA } \\
{[\mathrm{MHz}]}\end{array}$ \\
\hline $1 \mathrm{MHz}]$ & & {$[\%]$} \\
$228(13)$ & $26(13)$ & $0.57(11)$ & $51(1)$ \\
$4(10)$ & $21(10)$ & $0.28(8)$ & $46(1)$ \\
\hline
\end{tabular}

them.

In contrast to Fig. 6.49 a), Fig. 6.49 b) shows clearer oscillations in the perturbation function $R(t)$ and thus in its Fourier transform. The values derived from the analyis are given in Table 6.17. According to the literature [131], these parameters are similar to $\alpha-\mathrm{Cr}_{2} \mathrm{O}_{3}$. The high damping frequencies and thus the high asymmetry parameter $\eta$ are typical for amorphous materials or for materials with broad distributions in their EFGs. For a damping of $\delta \approx 20 \%$, the asymmetry parameter $\eta$ should be greater than 0 . If $\delta$ exceeds $35 \%, \eta$ can reach values grater than 0.5 . Thus, the observed $\eta$-values are consistent. Summarizing, it is shown that this new phase crystallizes and is sensitive to oxidation during even vacuum-annealing.

\subsubsection{Microhardness of nitrided stainless steel films}

Nanoindentation was performed by using a Fischerscope HV100 [92] with a Vickers diamond in order to investigate the influence of nitriding on the microhardness and the mechanical properties. The maximum indention force was set to $2 \mathrm{mN}$. Four positions were measured for each sample. The mean values are reported in Table 6.18 , where also the mean nitrogen content derived from RNRA is included.

By including data from [132], [133], a clear

Table 6.18: Hardness, Young modulus and Ncontent of nitrided AISI 316 films, where $E$ is the elastic modulus an $v$ the poisson ratio

\begin{tabular}{cccc}
\hline $\mathrm{N}_{2}$ Gas flow & Hardness & $\begin{array}{c}\text { Young modulus } \\
\mathrm{E} /\left(1-\mathrm{v}^{2}\right)\end{array}$ & N-content \\
{$[\mathrm{Gccm}]$} & {$[\mathrm{GPa}]$} & $77(9)$ & {$[$ at.\%] } \\
\hline 0.01 & $4.6(1)$ & $101(9)$ & $10(2)$ \\
0.05 & $6.2(1)$ & $103(10)$ & $18(2)$ \\
0.10 & $6.2(2)$ & $112(11)$ & $41(2)$ \\
0.50 & $6.8(1)$ & $91(8)$ & $48(2)$ \\
0.75 & $5.7(3)$ & $92(9)$ & $44(2)$ \\
1.00 & $5.8(2)$ & &
\end{tabular}

parabolic tendency between hardness and nitrogen 


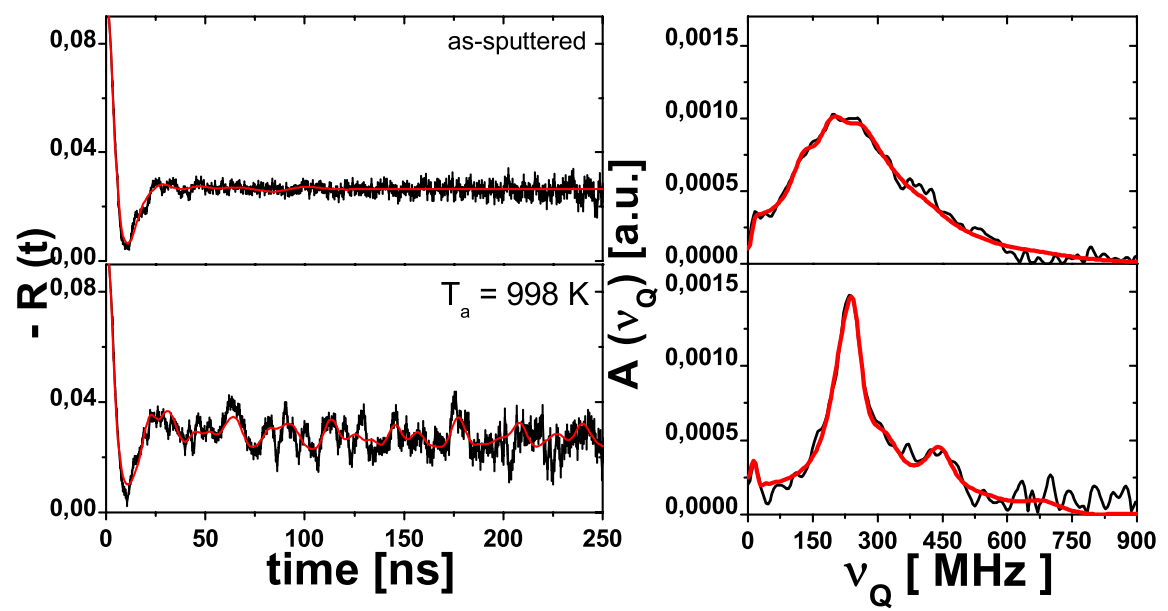

Figure 6.49: ${ }^{111}$ In PAC perturbation spectra along with their Fourier transforms for measurements of the as-nitrided samples: a) (top) PAC measurement of the as-nitrided sample sputtered at $0.05 \mathrm{sccm} \mathrm{N}_{2}$ gas flow and b) (bottom) PAC measurement after 2 hours of post vacuum annealing treatment performed at $T_{a}=973 \mathrm{~K}$. All presented spectra are recorded at $298 \mathrm{~K}$.

content can be seen, which is depicted in Fig. 6.50. The values obtained in this study are in good

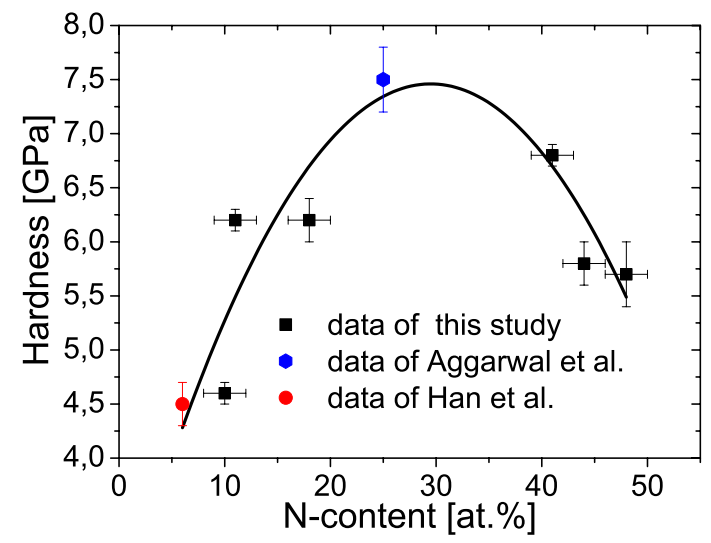

Figure 6.50: Dependency between Martens hardness and nitrogen content including data from [132], [133]. The $\mathrm{N}$ values are obtained from the RNRA analysis.

agreement to those reported in the literature. Nitrogen is known to occupy interstitial sites in AISI 316 which results in hardening [8]. Additional strengthening mechanisms are associated with the formation of nitrides. With involvement of the alloying elements in AISI 316, the reported values can be explained by $\mathrm{CrN} / \mathrm{Cr}_{2} \mathrm{~N}$ nitrides (which are difficult to identify in XRD) accompanied with the formation of $\epsilon-\mathrm{Fe}_{2} \mathrm{~N}-\mathrm{Fe}_{3} \mathrm{~N}$ nitrides.

The hardness value for the amorphous soft ferromagnetic phase is surprisingly high. For AISI 316, this phase exhibits a relative high hardness value.

\subsubsection{Discussion on a nucleation model for nitrided stainless steel films}

Similar to carburized samples, the experiments described above pose the following questions 1): how can the formation of the amorphous and soft ferromagnetic phase be explained. No. 2): How does the incorporation of nitrogen influence the phase formation, which leads to the question of the ruling phenomenon of nitride formation.

Also here, a combined model which refers to $\mathrm{Lu}$ et al. [13] and Lux and Haubner [113], so that the first question can be answered as follows: the present Fe-based alloy is associated with the deep eutectic point of the Fe-N system. It is well known that compositions around the deep eutectic point are ideal for glass formation in many systems. As a result, glass formation is greatly favored thermodynamically. Further, the minor addition of Mo could promote glass formation in the Fe-N system by suppressing the formation of the primary phase (i.e. Fe nitrides) and hindering grain growth. Because of their limited solubility in Fe nitrides, the molybdenum atoms must redistribute and long-range diffusion is required upon solidification. This is confirmed by RNRA, where the samples nitrided at $0.01-0.10 \mathrm{sccm} \mathrm{N}_{2}$ gas flow show a dramatic variation in nitrogen content. Thus, the minor addition of Mo could retard the nucleation process. Indeed, the given parameter range show amorphous XRD 
patterns.

The emerged phases above $0.10 \mathrm{sccm} \mathrm{N}_{2}$ gas flow can be explained by combining the mentioned model from Lu et al. with that of Lux and Haubner [113]: during the nucleation process nitronaceous species were adsorbed on the surface. Via vapor phase interaction, free $\mathrm{N}$ atoms were formed. Diffusion processes already inserted by Mo atoms solve the $\mathrm{N}$ atoms in the metal matrix, until nitrides like $\epsilon$ - $(\mathrm{Fe}, \mathrm{Cr})_{2} \mathrm{~N}$ and $(\mathrm{Fe}, \mathrm{Cr})_{3} \mathrm{~N}$ nitrides were formed. These phases were needed to form a closed nitride film, which then favors the formation of the FeN phase. This seems to be proven by RNRA, where the N-content at the beginning of the nucleation process start to increase until $\mathrm{N}$ values around 50 at.\% were reached. Also this is confirmed by XRD. The long range diffusion in the range of 0.50$1.00 \mathrm{sccm} \mathrm{N}_{2}$ gas flow appears to be less severe. According to Fig. 6.41, the growth rate reaches here higher values, which could retard the diffusion process. This leads then to the formation of nitride phases as seen by CEMS and XRD.

These two combined models explain the variety of the observed phases and the different nitrogen contents in the nitrided films and is further confirmed by Fig. 6.51, which shows similar to Fig. 6.50 a clear parabolic tendency: for nitrogen concentrations up

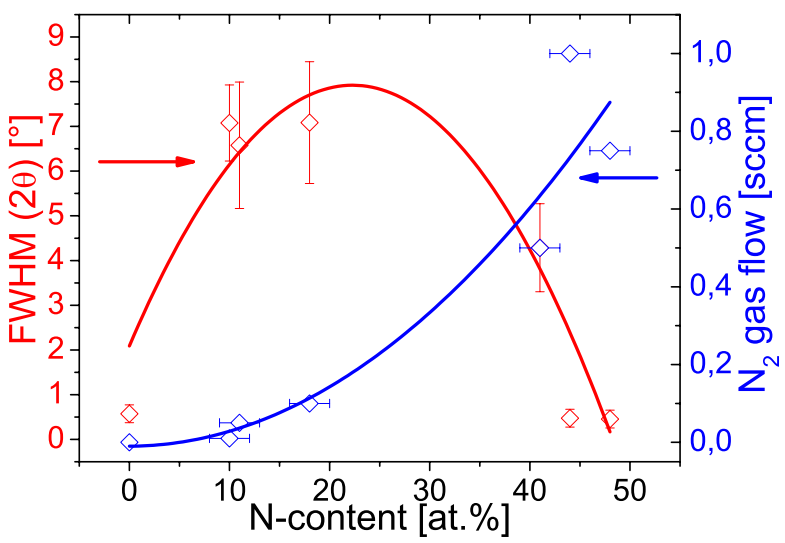

Figure 6.51: Peak width of the first amorphous peak and reactive gas flow in dependence of the nitrogen content of reactive sputtered stainless steel films.

to $25 \%$, the peak width increases. At $25 \% \mathrm{~N}$ content, the stoichiometry of $\mathrm{Fe}_{3} \mathrm{~N}$, Fig. 6.51 has its maximum and starts then to decrease, until it reaches the parameter of the FeN phase. Simultaneously, the gas flow increases exponential from 0.01 to $1.00 \mathrm{sccm} \mathrm{N}_{2}$ gas flow. Both Figures, Fig. 6.50 and Fig. 6.51, have the form of a Gibbs plot, wherein the parameter range exhibits the $\mathrm{Fe}_{3} \mathrm{~N}$ as minimum, and thus, as thermodynamically favored phase. Combined with the shown tendency obtained for the reactive gas flow, any given phase from amorphous to crystalline - can be reached for magnetron-nitrided stainless steel films.

The similarity of the amorphous states observed in carburized and nitrided samples lead to conclusion, that in both cases the underlying mechanism is the same. Thus, the magnetism in the amorphous and soft ferromagnetic phase (sputtered at $0.05 \mathrm{sccm} \mathrm{N}_{2}$ gas flow) is due to nano-crystalline grains consisting of a disordered $\mathrm{Ni}_{3} \mathrm{~N}$ phase surrounded by a spin glassy surface shell.

To investigate, if the found similarity of the observed amorphous states are based on a generalized model, which is independent of the used reactive gas, experiments with oxygen were carried out. The results were presented in the next section. 


\subsection{Results for oxidized stain- less steel films}

The films were sputter-deposited with an rf magnetron onto amorphous $\mathrm{SiO}_{2}$ substrates (oxidized $\mathrm{Si}(100)$ wafer of $0.5 \mathrm{~mm}$ thickness, pre-cleaned with acetone and oxidized in air, no further treatment) utilizing a commercial AISI 316 (X5CrNiMo17-12$2,1.4401)$ sputter-target. The target-substrate distance was set to $10 \mathrm{~cm}$. The processing parameters were always $100 \mathrm{~W}$ magnetron power at a constant total gas flow of $12 \mathrm{sccm}$ during deposition. Several sample series were deposited at different $\mathrm{O}_{2}$ flows (0.00-1.00 sccm). The sputter rate was always in the range of of $0.10-0.25 \mathrm{~nm} / \mathrm{s}$ and was depending on the gas flow parameters. The deposition procedure were carried out in the same way as for carburized and nitrided stainless steel films. The deposition parameters and the resulting thicknesses and growth rates are summarized in Table 6.19.

From these data it is derived that the growth

Table 6.19: Gas flow $j$, deposition time $t$, real film thickness $d$ (as measured by Rutherford Backscattering Spectrometry (RBS) after deposition) and derived growth rate $g=\frac{d}{t}$ for the deposited oxidized films. All samples were deposited at $298 \mathrm{~K}$ with a magnetron power of $100 \mathrm{~W}$ and a targetsubstrate distance of $10 \mathrm{~cm}$.

\begin{tabular}{cccccr}
\hline sample & $\begin{array}{c}j_{\mathrm{O}_{2}} \\
{[\mathrm{sccm}]}\end{array}$ & $\begin{array}{c}j_{\mathrm{Ar}} \\
{[\mathrm{sccm}]}\end{array}$ & $\begin{array}{c}t \\
{[\mathrm{~min}]}\end{array}$ & $\begin{array}{c}d \\
{[\mathrm{~nm}]}\end{array}$ & $\begin{array}{r}g \\
{[\mathrm{~nm} / \mathrm{min}]}\end{array}$ \\
\hline O0 & 0.00 & 12.00 & $17: 00$ & $205(10)$ & $12.06(92)$ \\
O1 & 0.01 & 11.99 & $16: 55$ & $260(10)$ & $15.37(118)$ \\
O5 & 0.05 & 11.95 & $15: 42$ & $269(10)$ & $17.13(135)$ \\
O10 & 0.10 & 11.90 & $17: 09$ & $362(10)$ & $21.11(138)$ \\
O50 & 0.50 & 11.50 & $20: 57$ & $92(10)$ & $4.39(53)$ \\
O75 & 0.75 & 11.25 & $25: 13$ & $65(10)$ & $2.58(41)$ \\
O100 & 1.00 & 11.00 & $26: 44$ & $469(10)$ & $17.54(79)$ \\
\hline
\end{tabular}

rate has a sinusoidal form (Fig. 6.52), which was also seen for nitrided samples [134]. The first maximum of the growth rate characteristic ca be found at $0.10 \mathrm{sccm}$, its second maximum at $1.00 \mathrm{sccm} \mathrm{O}_{2}$ gas flow. As be seen below, these parameters represent two metastable phases: a nano-crystallized cubic $\mathrm{FeO}$ phase (@ $0.10 \mathrm{sccm} \mathrm{O}_{2}$ gas flow) and an amorphous and soft ferromagnetic phase rich in oxygen (@1.00 sccm $\mathrm{O}_{2}$ gas flow).

\subsubsection{Deposition at room tempera- ture and influence of the oxy- gen gas flow}

The oxygen gas flow was systematically increased from $0.01 \mathrm{sccm}$ to $1.00 \mathrm{sccm}$. For all these deposited films, the reactive magnetron deposited

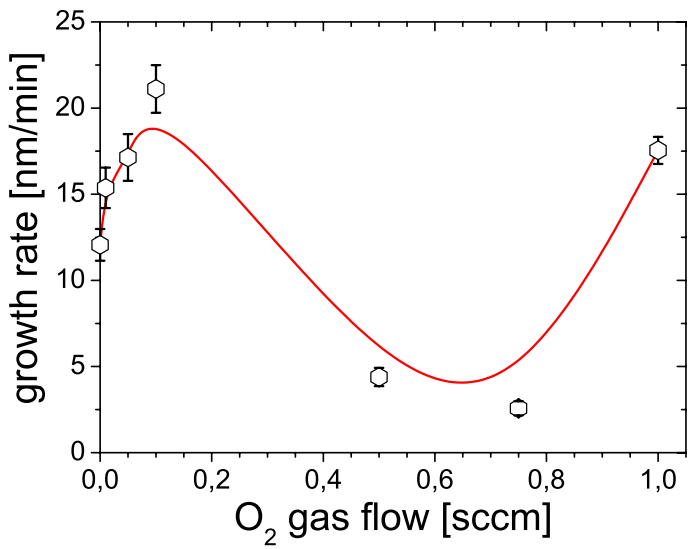

Figure 6.52: Growth rate $g$ as a function of the $\mathrm{O}_{2}$ gas flow $j_{\mathrm{O}_{2}}$ for the room temperature deposition (line is only to guide the eye).

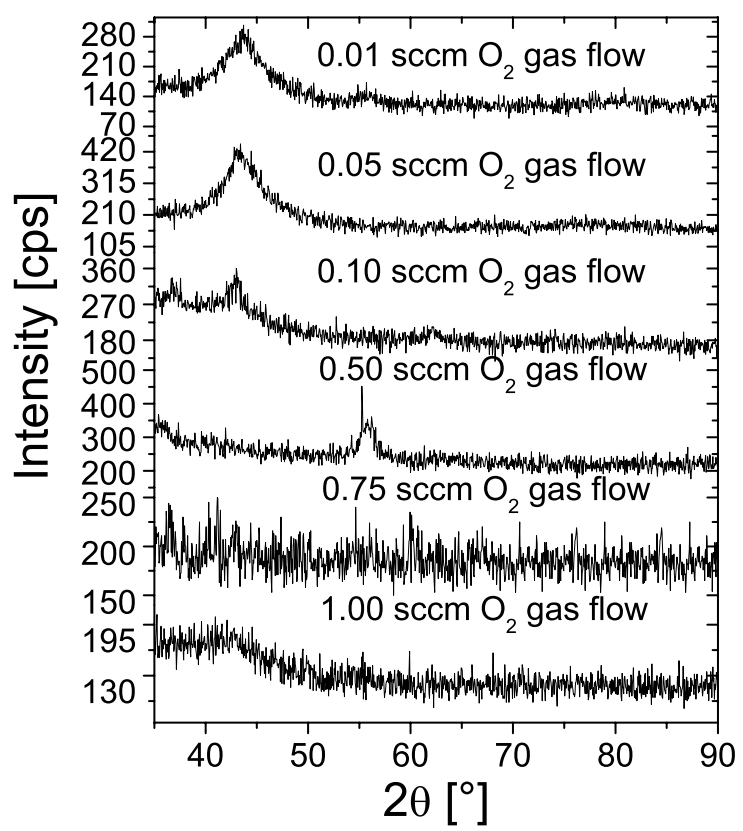

Figure 6.53: GIXRD $\left(2^{\circ}\right)$ spectra of the oxidized films. The $\mathrm{O}_{2}$ gas flows are given in the graph.

and oxidized samples show the typical broad XRD appearance of amorphous materials, as seen in Fig. 6.53. These diffractograms show a clear amorphous signature.

Fig. 6.55 shows the position and the width of the first broad peak for the spectra shown in Fig. 6.53. There is a clear tendency for peak position and peak width; both, peak position and peak width, have a parabolic form with its minimum at 0.75 $\mathrm{O}_{2}$ gas flow. 

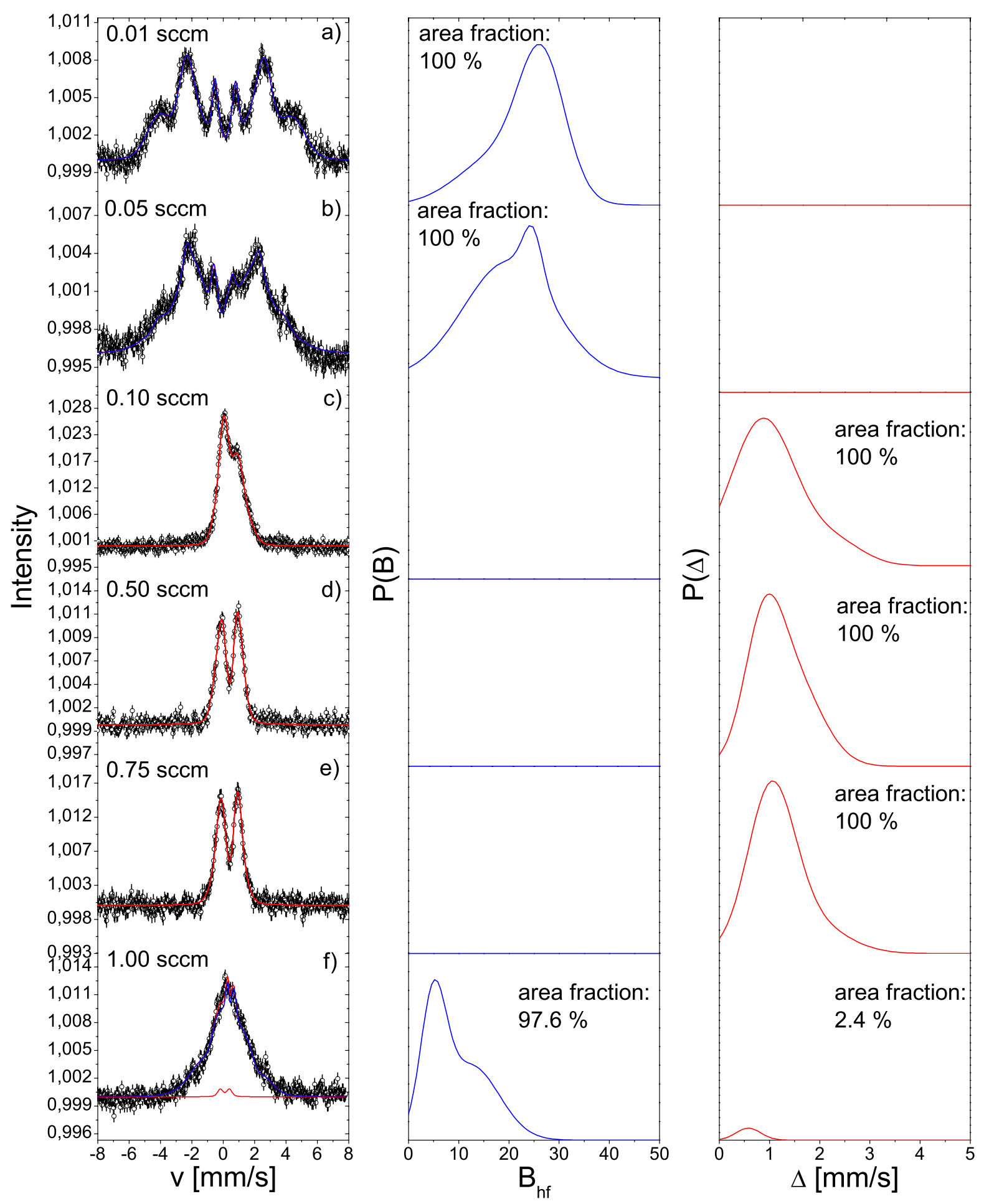

Figure 6.54: Mössbauer spectra of the reactive sputtered AISI 316 films. The numbers in the graphs represent the oxygen gas flow. 


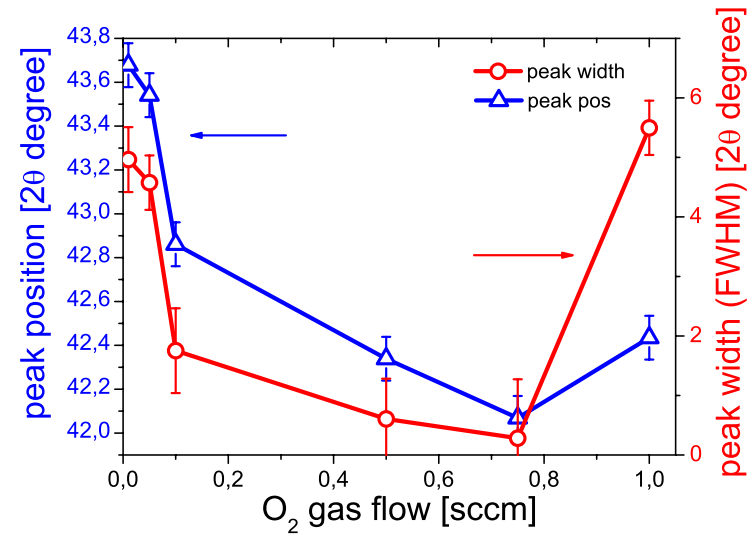

Figure 6.55: Peak position and peak width of the first broad peak in the XRD spectra of the reactive sputtered stainless steel films.

Fig. 6.54 summarizes the CEMS measurements of the samples with increasing gas flow. They show the typical Mössbauer spectra of amorphous materials. Spectra in a) and b) are magnetically split, the spectrum in c) reveals the $(\mathrm{Fe}, \mathrm{Cr}, \mathrm{Ni}) \mathrm{O}$ phase, whereas d) and e) represent spectra for $\gamma$ $(\mathrm{Fe}, \mathrm{Cr}, \mathrm{Ni})_{2} \mathrm{O}_{3}$. Finally, spectrum f) exhibits the quasi-metallic glass phase, which was also observed for carburized and nitrided samples. The difference between the quasi-metallic phase sputtered with an oxygen atmosphere and those sputtered with methane and nitrogen is the relative high isomer shift. Whereas the the carburized and nitrided quasi-metallic glass sample have a relative insignificant isomer shift, the oxidized sample reveals an isomer shift of $\delta \sim 0.30 \mathrm{~mm} / \mathrm{s}$. This indicates a participation of iron atoms with a high oxidation state (i.e. Fe oxides). The fitted hyperfine parameters are presented in Table 6.20.

To confirm the magnetic properties obtained by Mössbauer Spectroscopy, MOKE measurements were carried out. While the Fig. 6.54 a) and b) show a magnetically split sextet, no MOKE-signal is obtained for these samples. Due to the information depth of MOKE (5-10 nm), two explanation are possible: 1) there could be non-magnetic Fe-oxides on the surface and 2) the whole sample is antiferromagnetic. Since there is no noticeable amount of additional doublets in the spectra, the second explanation is more probable. As a consequence, only the quasi-metallic phase sputtered at $1.00 \mathrm{sccm} \mathrm{O}$ gas flow exhibits a MOKE signal. The results are illustrated in Fig. 6.56.

A clear fourfold anisotropy can be observed, which could be due to magnetostriction effects, stresses or by a phase transition to a $\gamma$ phase $[88,89,111]$. The coercive field $H_{C}$ is approximately 6 Oe.

The EDX analyses of the deposited films revealed

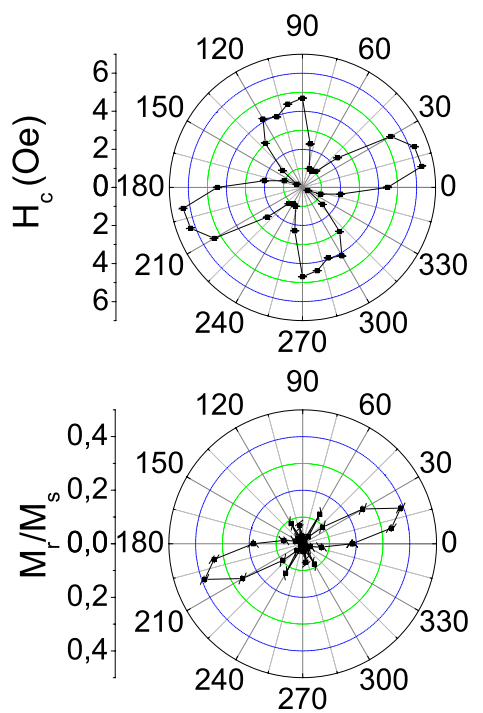

Figure 6.56: Angular scans of the deposited film sputtered at $1.00 \mathrm{sccm} \mathrm{O}_{2}$ gas flow. The polar diagrams of the coercive field $H_{\mathrm{C}}$ (top) and the relative remanence $M_{\mathrm{R}} / M_{\mathrm{S}}$ (bottom) are shown.

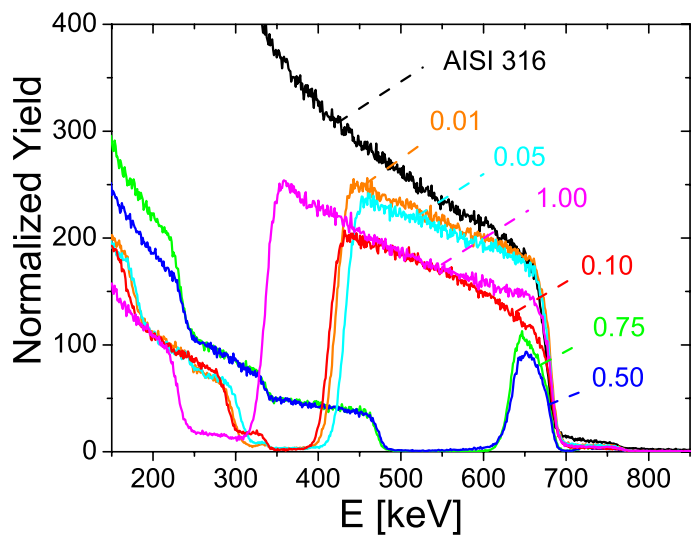

Figure 6.57: RBS spectra of the reactively sputtered films. The $\mathrm{O}_{2}$ flow is given in the graph.

the original composition of the sputtering targets within the experimental limits. Therefore, in order to evaluate the amount of incorporated nitrogen, RBS measurements were carried out, whose results are shown in Fig. 6.57 and can reach almost $72(2)$ at. $\%$.

In Fig. 6.58 the oxygen content derived from the RBS analysis can be seen. Also here a clear parabolic tendency can be seen with increasing $\mathrm{O}_{2}$ gas flow. The maximum regions for the samples sputtered with 0.50 and 0.75 sccm correspond, with respect to their oxygen content, to $\mathrm{Fe}_{2} \mathrm{O}_{3}$ oxides. Since no magnetism can be observed in Mössbauer Spectroscopy and MOKE, these oxides can be iden- 
Table 6.20: Mössbauer fitting results of the nitrided AISI 316 samples, deposited at room temperature with a magnetron power of $100 \mathrm{~W}(f$ - area fraction (error), mean values of $\langle\delta\rangle$ - isomer shift, $\langle\Delta\rangle-$ the quadrupole splitting for the paramagnetic subspectra, $\langle\epsilon\rangle$ the quadrupole splitting for the magnetic subspectra, $B$ - hyperfine field).

\begin{tabular}{ccrrrrrr}
\hline $\begin{array}{c}\mathrm{N}_{2} \\
{[\mathrm{sccm}]}\end{array}$ & part & $\begin{array}{r}f \\
{[\%]}\end{array}$ & $\begin{array}{r}\langle\delta\rangle \\
{[\mathrm{mm} / \mathrm{s}]}\end{array}$ & $\begin{array}{r}\langle\Delta\rangle,\langle\epsilon\rangle \\
{[\mathrm{mm} / \mathrm{s}]}\end{array}$ & $\begin{array}{r}\sigma_{\Delta} \\
{[\mathrm{mm} / \mathrm{s}]}\end{array}$ & $\begin{array}{r}\langle B\rangle \\
{[\mathrm{T}]}\end{array}$ & $\begin{array}{r}\sigma_{B} \\
{[\mathrm{~T}]}\end{array}$ \\
\hline 0.01 & ferro-1 & $40.0(53)$ & 0.13 & 0.01 & & $18.0(11)$ & $8.4(41)$ \\
& ferro-h & $60.0(23)$ & 0.13 & 0.01 & & $26.6(6)$ & $4.6(12)$ \\
\hline 0.05 & ferro-1 & $91.8(37)$ & -0.08 & -0.05 & & $20.0(5)$ & $9.1(6)$ \\
& ferro-h & $8.2(48)$ & -0.08 & -0.05 & & $24.7(6)$ & $1.9(9)$ \\
\hline 0.10 & para-1 & $89.3(39)$ & 0.48 & 0.88 & 0.65 & & \\
& para-2 & $10.7(43)$ & & 2.35 & 0.54 & & \\
\hline 0.50 & para-1 & $47.6(19)$ & 0.40 & 0.88 & 0.38 & & \\
& para-2 & $52.4(10)$ & & 1.50 & 0.57 & & \\
\hline 0.75 & para-1 & $77.7(54)$ & 0.38 & 1.03 & 0.47 & & \\
& para-2 & $22.3(44)$ & & 1.80 & 0.70 & & \\
\hline 1.00 & ferro-1 & 42.2 & 0.33 & 0.30 & & $4.9(3)$ & $9.1(6)$ \\
& ferro-h & 55.4 & 0.33 & 0.30 & & $12.4(26)$ & $5.7(17)$ \\
& para-1 & $2.4(4)$ & 0.1 & 0.58 & 0.25 & & \\
\hline
\end{tabular}

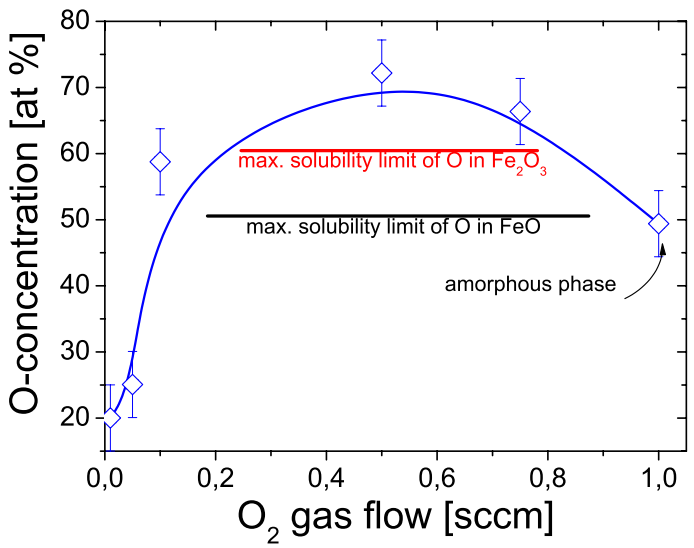

Figure 6.58: Oxygen content in the reactively sputtered films as derived from the RBS analysis versus the $\mathrm{O}_{2}$ gas flow.

tified as $\gamma-\mathrm{Fe}_{2} \mathrm{O}_{3}$ oxides.

\subsubsection{PAC characterization of the vacuum annealed soft ferro- magnetic phase}

To study the influence of vacuum annealing of the amorphous and soft ferromagnetic phase found at $1.00 \mathrm{sccm} \mathrm{O}_{2},{ }^{111} \mathrm{In}$ atoms were implanted at an energy of $400 \mathrm{keV}$ at the $530 \mathrm{kV}$ IONAS accelerator facility in Göttingen and then carefully analyzed by PAC at 10 and $298 \mathrm{~K}$. After anlayzing the as-oxidized sample, it was vacuum annealed $\left(p_{a}=10^{-3} \mathrm{~Pa}\right)$ at $973 \mathrm{~K}$ for 2 hours. The results of the PAC measurement are shown in Fig. 6.59. The spectra measured at $10 \mathrm{~K}$ did not show any changes in comparison to those which were recorded at $298 \mathrm{~K}$. Therefore, only the spectra recorded at
Table 6.21: PAC interaction parameters of the annealed amorphous and soft ferromagnetic phase at $973 \mathrm{~K}$ for 2 hours shown in Fig. $6.59 \mathrm{~b}$ ). Hyperfine parameters: $\nu_{Q}$ is the quadrupole interaction frequency, $\delta$ the damping of $\nu_{Q}, \eta$ the asymmetry parameter, and RA the relative area.

\begin{tabular}{cccc}
\hline$\nu_{Q}$ & $\delta$ & $\eta$ & $\begin{array}{l}\text { RA } \\
{[\%]}\end{array}$ \\
\hline $296(13)$ & $4(1)$ & 0.00 & $6(2)$ \\
$210(17)$ & 0 & $0.26(11)$ & $7(6)$ \\
$177(13)$ & $55(5)$ & $0.56(21)$ & $84(12)$ \\
\hline
\end{tabular}

$298 \mathrm{~K}$ for the as-oxidized and vacuum annealed sample are shown in Fig. 6.59. As the PAC spectra give no indication of a magnetic hyperfine field, they have been fitted assuming an EFG-distribution.

Fig. 6.59 a) shows the typical behavior of amorphous materials, where the PAC probe has no defined position in the matrix due to the absence of crystalline order. As a consequence, the quadrupole interaction frequency $\nu_{Q}$ shows a broad frequency distribution caused by randomly excited EFGs (note: this system consists of five elements and thus, there are 14 combinations of pair interactions). No further information can be taken from them.

In contrast to Fig. 6.59 a), Fig. 6.59 b) shows clearer oscillations in the perturbation function $R(t)$ and thus in its Fourier transform. The values derived from the analyis are given in Table 6.21.

According to the literature [131], these parameters are similar to $\alpha-\mathrm{Cr}_{2} \mathrm{O}_{3}$. In contrast to the PAC results of the nitrided samples, the damping frequencies are very small, except of that for the $177 \mathrm{MHz}$ frequency, however, the high asymmetry parameter $\eta$ are typical for amorphous materials or for materials with broad distributions in their EFGs. For a damping of $\delta \approx 20 \%$, the asymmetry parameter $\eta$ should be greater than 0 . If $\delta$ exceeds $35 \%, \eta$ can 

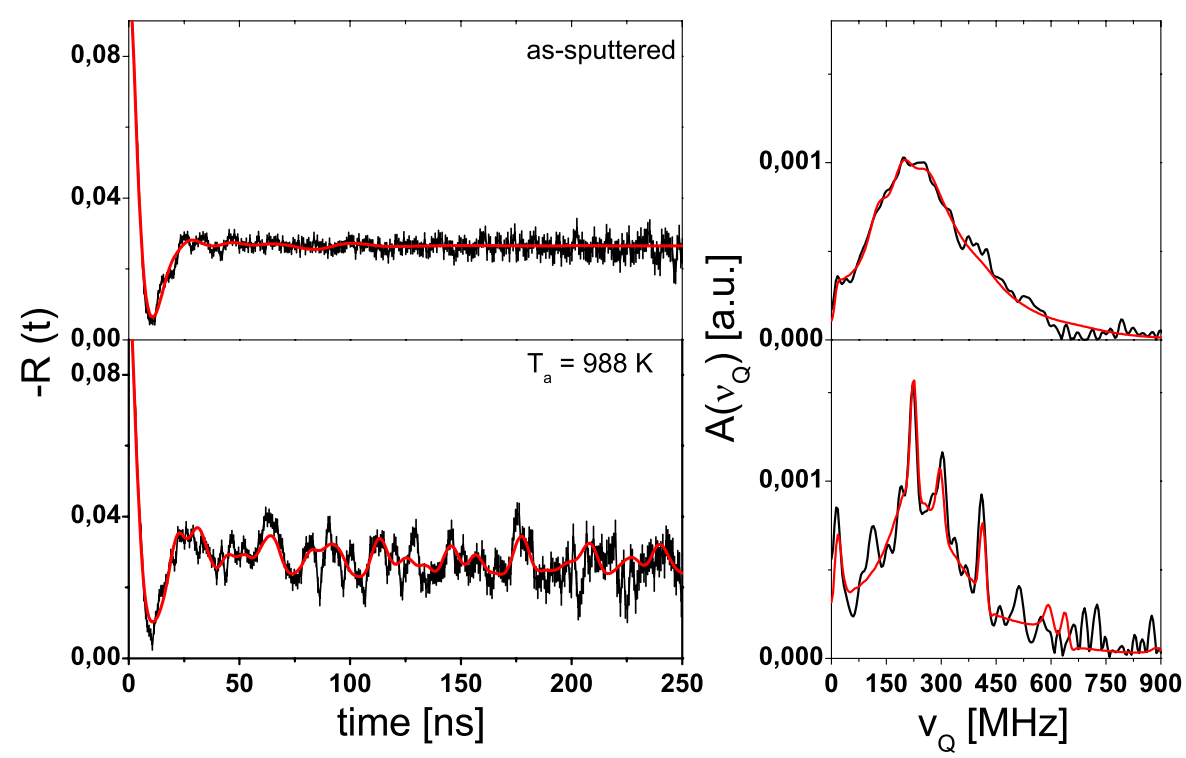

Figure 6.59: ${ }^{111}$ In PAC perturbation spectra along with their Fourier transforms for measurements: a) (top) PAC measurement of the as-oxidized sample sputtered at $1.00 \mathrm{sccm} \mathrm{O}_{2}$ gas flow and b) (bottom) PAC measurement after 2 hours of post vacuum annealing treatment performed at $T_{a}=973 \mathrm{~K}$. All presented spectra are recorded at $298 \mathrm{~K}$.

reach values grater than 0.5 . Thus, the observed $\eta$ values are consistent. Summarizing, it is shown that this new phase crystallizes and is sensitive to oxidation during even vacuum-annealing and is in its annealing behavior verisimilar to the quasi-metallic glass phase obtained after nitriding.

\subsubsection{Microhardness of oxidized stainless steel films}

Nanoindentation was performed by using a Fischerscope HV100 [92] with a Vickers diamond in order to investigate the influence of nitriding on the microhardness and the mechanical properties. The maximum indention force was set to $2 \mathrm{mN}$. Four positions were measured for each sample. The mean values are reported in Table 6.22.

Fig. 6.60 shows the hardness as a function of oxy-

Table 6.22: Hardness, Young modulus and Ocontent of oxidized AISI 316 films, where $E$ is the elastic modulus an $v$ the poisson ratio

\begin{tabular}{cccc}
\hline $\mathrm{O}_{2}$ Gas flow & Hardness & $\begin{array}{c}\text { Young modulus } \\
\mathrm{E} /\left(1-\mathrm{v}^{2}\right) \\
{[\mathrm{GPa}]}\end{array}$ & $\begin{array}{c}\text { O-content } \\
{[\mathrm{sccm}]}\end{array}$ \\
{$[\mathrm{GPa}]$} & $92(4)$ & $20(2)$ \\
\hline 0.01 & $5.8(1)$ & $92(5)$ & $25(2)$ \\
0.05 & $5.7(1)$ & $94(3)$ & $59(2)$ \\
0.10 & $6.0(2)$ & $119(18)$ & $72(2)$ \\
0.50 & $6.8(1)$ & $73(2)$ & $66(2)$ \\
0.75 & $6.5(3)$ & $128(12)$ & $50(2)$ \\
1.00 & $6.9(2)$ & & \\
\hline
\end{tabular}

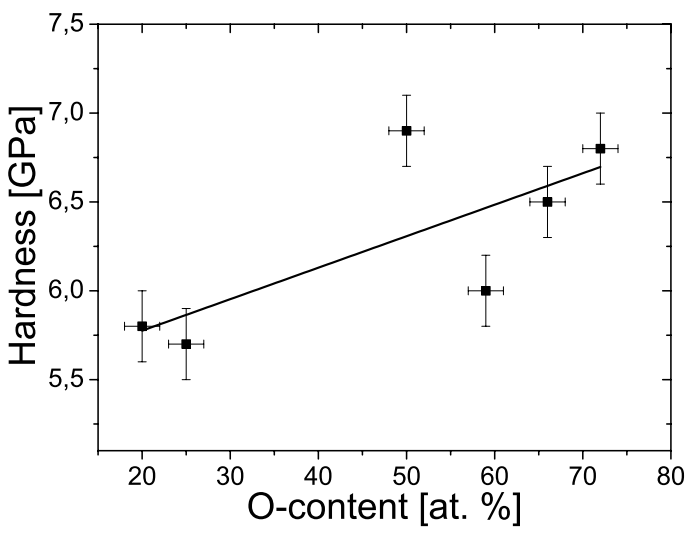

Figure 6.60: Dependency between Martens hardness and oxygen content. The Oxygen values are obtained from the RBS analysis.

gen content. A clear tendency can be seen, where the hardness of the films increase linearly with increasing oxygen content and follows the law:

$$
y=0.02(1) x+5.42(22) \mathrm{GPa}
$$

It seems, that the hardness of the films is only influenced by the incorporation of oxygen independently of the formed phase.

The highest oxygen contents are observed for the samples sputtered at $0.50,0.7 \mathrm{sccm} \mathrm{O}_{2}$ gas flow - i.e. samples containing $\mathrm{Fe}_{2} \mathrm{O}_{3}$ oxides - and the amorphous and soft ferromagnetic phase sputtered 
at $1.00 \mathrm{sccm} \mathrm{O}_{2}$ gas flow. Besides Mössbauer Spectroscopy, this is another hint that the formation of the amorphous and soft ferromagnetic phase is influenced by the formation of oxides.

\subsubsection{Discussion on a nucleation model for oxidized stainless steel films}

Similar to carburized and nitrided samples, following questions arise: 1) how can the formation of the amorphous and soft ferromagnetic phase be explained and 2): how does the incorporation of oxygen influence the phase formation and what is the ruling phenomenon of oxide formation.

Also here, a combined model which refers to Lu et al. [13] and Lux and Haubner [113], so that the first question can be answered as follows: the present Fe-based alloy is associated with the deep eutectic point of the Fe-O system. It is well known that compositions around the deep eutectic point are ideal for glass formation in many systems. As a result, glass formation is greatly favored thermodynamically. Further, the minor addition of Mo could promote glass formation in the $\mathrm{Fe}-\mathrm{O}$ system by suppressing the formation of the primary phase (i.e. Fe oxides) and hindering grain growth. Because of their limited solubility in Fe oxides, the molybdenum atoms must redistribute and long-range diffusion is required upon solidification. Thus, the minor addition of Mo could retard the nucleation process. Indeed, all XRD patterns show the behavior of amorphous materials.

The incorporation of oxygen can be explained by

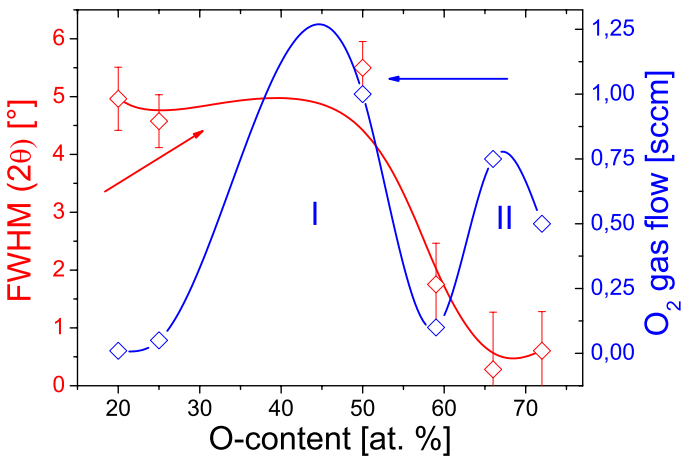

Figure 6.61: Peak width of the amorphous peak and reactive gas flow in dependence of the oxygen content of reactive sputtered stainless steel films.

combining the mentioned model from Lu et al. with that of Lux and Haubner [113]: during the nucleation process oxynaceous species were adsorbed on the surface. Via vapor phase interaction, free $\mathrm{O}$ atoms were formed. Diffusion processes already inserted by Mo atoms solve the $\mathrm{O}$ atoms in the metal matrix. This explains the relative low $\mathrm{O}$ content in the parameter range up to $0.05 \mathrm{sccm} \mathrm{O}_{2}$ gas flow, wherein stoichiometric oxides were formed only locally. With increasing growth rate, the oxygen take up is enough to form oxides globally in the films. At $1.00 \mathrm{sccm} \mathrm{O}_{2}$ gas flow, the growth rate achieves its maximum (and a similar value as for samples sputtered at low $\mathrm{O}_{2}$ gas flow), the parameter for the amorphous and soft ferromagnetic phase.

For small and for high $\mathrm{O}_{2}$ gas flows, the samples appear amorphous (which can be seen for the developing FWHM in Fig. 6.61), whereas samples sputtered in the range of $0.1-0.75 \mathrm{sccm} \mathrm{O}_{2}$ gas flow begin to crystallize. If the $\mathrm{O}_{2}$ gas flow and the FWHM is arranged according to ascending oxygen content, Fig. 6.61 can be understood as a Gibbs plot of a multi-phase system, in which the transition from the amorphous state (domain I) to the crystalline phases (domain II - the formation of $\mathrm{FeO}, \mathrm{Fe}_{2} \mathrm{O}_{3}$ oxides) is depicted. Therefor, the growth rate in Fig. 6.52 is confirming this hypothesis, in which the growth rate of crystalline and amorphous samples are verisimilar. The minima of the systems are the quasi metallic glass and the $\mathrm{FeO} / \mathrm{Fe}_{2} \mathrm{O}_{3}$ phase.

Due to the similarity of the amorphous states, carburized and nitrided samples were described by a core-shell model, describing the nanoparticles as consisting of a ferromagnetically $\mathrm{Ni}_{3} \mathrm{C}$ core and a disordered surface shell. By contrast, Mössbauer Spectroscopy and RBS indicate the participation of Fe-oxides. As a consequence, the model for the amorphous state for carburized and nitrided samples can not be applied in the same form for oxidized samples.

According to $\mathrm{He}$ et al. [118] and Kodama et al. [135], the previous model is also applicable to different ferromagnetically cores, e.g. also for $\mathrm{NiFe}_{2} \mathrm{O}_{4}$. Since $\mathrm{Ni}$ is a crucial for phase formation in stainless steels $[7,91,129]$, it is reasonable to assume that the amorphous state observed for the sample sputtered at $1.00 \mathrm{sccm} \mathrm{O}_{2}$ gas flow can be described by a multi-shell model consisting of a ferromagnetically $\mathrm{NiFe}_{2} \mathrm{O}_{4}$ core surrounded by a spin-glassy surface layer. This would be in good agreement to RBS and especially Mössbauer, where the isomer shift $\delta \approx 0.3 \mathrm{~mm} / \mathrm{s}$ indicate a Fe-oxide participation in phase formation.

Finally, EXAFS investigations on oxidized samples are required to resolve the microstructure of oxidized Magnetron-sputtered samples. 


\subsubsection{Summary of the amorphization process and nucleation model of Magnetron-sputtered stain- less steel films}

Various aspects of the carbide, nitride and oxides formation and their stability in reactive magnetronsputtered austenitic stainless-steel films were studied.

Intrigued by the predictions of Lee et al. [9], inert sputtering from a pre-combined $\mathrm{Fe}_{50} \mathrm{C}_{50}$ was used to synthesize the stoichiometric $\mathrm{NaCl}$-type $\mathrm{FeC}$ phase. Unfortunately, geometric effects of the target configuration, hysteresis effects and re-sputtering constitute severe problems to the deposition process. As a consequence of this, the Magnetron sputtering technique seems not to be suitable to synthesize the stoichiometric FeC phase, but the films exhibit carbon contents, which exceed the maximum solubility limits in known carbides. As a result, reactive sputtering of stainless steel - by using methane, nitrogen and oxygen as reactive gas - was performed to synthesize amorphous films or quasi metallic glasses based on conventional steels.

All carburized stainless steel films revealed amorphous character and the magnetic properties investigated by means of Mössbauer Spectroscopy and MOKE showed the formation of various phases and carbides at a sputtering temperature of $298 \mathrm{~K}$. In addition, a new amorphous soft ferromagnetic phase was observed. Vacuum annealing of this phase showed the carbide reaction $\mathrm{M}_{7} \mathrm{C}_{3} \rightarrow$ $\mathrm{M}_{23} \mathrm{C}_{6} \rightarrow \mathrm{M}_{6} \mathrm{C}$ and confirmed the existence of a stable Fe-graphite and the metastable Fe-cementite system [136-138].

By using low gas fluxes, nitrided samples also showed the formation of films with typical amorphous behaviors and also the new amorphous soft ferromagnetic phase was observed. For this samples, RNRA showed extremely differing N-depth profiles. In contrast, high fluxes revealed the formation of a cubic ZnS-type $\gamma^{\prime \prime}-(\mathrm{Fe}, \mathrm{Cr}, \mathrm{Ni}) \mathrm{N}$ phase and their depth profiles were, as expected, constant. Noticeable is that the investigated $\mathrm{Fe}$ /stainless steel-N system behaves like a perfect Gibbs-system with the $\mathrm{Fe}_{3} \mathrm{~N}$ phase as minimum.

Oxidized samples showed amorphous character over the whole range of the processing parameters, also including the new amorphous soft ferromagnetic phase, but showed appendages of crystallization as indicated by Mössbauer and XRD. These crystallites could be identified as $\mathrm{Fe}_{2} \mathrm{O}_{3}$ oxides. A PAC study, which was also performed on nitrided samples, revealed, that this new phase crystallizes and is even sensitive to oxidation during vacuumannealing. Noticeable is that the Fe/stainless steel-
O system can be understood as a Gibbs multi-phase system, in which the transition from the amorphous state to the crystalline phases can be observed.

Fig. 6.62 shows the dependence of the normalized gas flow, which is needed to form the new amorphous soft ferromagnetic phase, as a function of the radius of the reactive gas atoms. A clear tendency can be seen, where the normalized flow decreases exponentially with increasing reactive gas radius.

The most important parameters to obtain this new

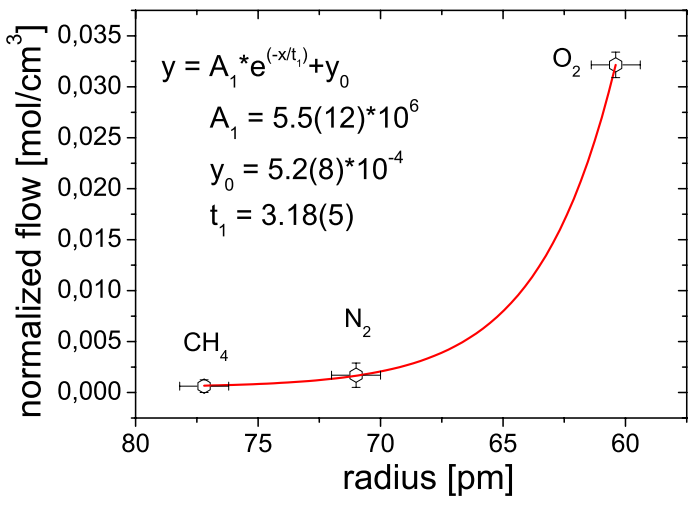

Figure 6.62: Normalized gas flow in dependence of the radius of the reactive gas atoms. The parameters for the exponential decay fitting routine are given in the graph.

amorphous and soft ferromagnetic phase are given in Table 6.23.

To characterize the microstructure of the new amorphous and soft ferromagnetic phase, DSC, FIM, TEM and EXAFS experiments were carried out for carburized samples.

DSC, FIM and TEM showed the quasi metallic glass behavior of these phase, but the microstructure could only be explained by the EXAFS analysis, which revealed a disordered $\mathrm{Ni}_{3} \mathrm{C}$ phase. The magnetism of this phase could be predicted by a LMTO model [119], the order of magnetism could be explained by a core-shell model, describing the nano-particles as consisting of a ferromagnetically core and a disordered surface shell $[121,122]$.

These experiments confirmed a pre-suggested model, which is based on thermodynamical and kinetic remarks of Lu et al. [13] and of Lux and Haubner [113]: the present Fe-based alloy is associated with the deep eutectic point of the Fe-X system. It is well known that compositions around the deep eutectic point are ideal for glass formation in many systems. As a result, glass formation is greatly favored thermodynamically. Further, the minor addition of Mo could promote glass formation in the $\mathrm{Fe}-\mathrm{X}$ system by suppressing the formation of the primary phase and hindering grain growth. Be- 
Table 6.23: Summary of the synthesization parameters of the new amorphous and soft ferromagnetic phase: $R$ is the theoretical calculated radius, $R_{I}$ the ionic or van-der-Waals radius, $R_{C}$ the covalent radius, $\sigma_{50: 50}$ the structure of stoichiometric FeX phase (where $X$ represent the reactive gas atom), T $\sigma_{50: 50}$ the temperature at which the stoichiometric phase can be formed and $\Lambda$ the amorphization parameter.

\begin{tabular}{lccc}
\hline & $\mathrm{C}$ & $\mathrm{N}$ & $\mathrm{O}$ \\
\hline$R[\mathrm{pm}]$ & 77.2 & 71 & 60.4 \\
$R_{I}[\mathrm{pm}]$ & $20(2)$ & $170(2)$ & $133(1)$ \\
$R_{C}[\mathrm{pm}]$ & 77 & 70 & 66 \\
$\sigma_{50: 50}$ & $\mathrm{NaCl}-\mathrm{FeC}$ & $\mathrm{c}-\mathrm{FeN}$ & $\mathrm{c}-\mathrm{FeO}$ \\
$\mathrm{T}_{\sigma_{50: 50}}[\mathrm{~K}]$ & 298 & $298-573$ & $298 / 858-1673$ \\
$\Lambda$ & a-AISI $316+\mathrm{CH}_{4}$ & a-AISI $316+\mathrm{N}_{2}$ & $\mathrm{a}-\mathrm{AISI} 316+\mathrm{O}_{2}$ \\
& $>60 \% \mathrm{C}-0.01 \mathrm{sccm} \mathrm{CH}_{4}$ & $\sim 18 \% \mathrm{~N}-0.05 \mathrm{sccm} \mathrm{\textrm {N } _ { 2 }}$ & $\sim 50 \% \mathrm{O}-1.00 \mathrm{sccm} \mathrm{O}_{2}$ \\
\hline technique & Magnetron/PLD & Magnetron & Magnetron \\
\hline
\end{tabular}

cause of their limited solubility in iron bases anions, sults were presented in the next sections. the molybdenum atoms must redistribute and longrange diffusion is required upon solidification. The introduction of a reactive sputtering gas additionally disturbs the nucleation process by establishing higher probabilities of coordination. This leads to simultaneous rearrangement of different species of atoms, which suppresses the formation of competing ordered phases. As a consequence, phases outside the equilibrium can be formed like the disordered trigonal $\mathrm{Ni}_{3} \mathrm{C}$ phase.

The incorporation of the reactive sputter gas atoms can be described as follows: during the nucleation process the dissociated reactive sputter gas molecules were adsorbed on the surface. Via vapor phase interaction, free atoms were formed. Diffusion processes already inserted by Mo atoms solve the sputter atoms in the metal matrix depending on the processing parameters.

Due to the presence of isomorphic phases in nitrided samples and the fact, that the new phase almost has the same hyperfine parameters, it is assumed that the origin of magnetism in this phase succumbs the same phenomenon - a disordered $\mathrm{Ni}_{3} \mathrm{C}$ phase surrounded by a spin-glassy surface shell.

In contrast to the carburized and nitrided quasi metallic phase, Mössbauer Spectroscopy and RBS of the oxidized phase indicated the participation of Fe-oxides. As a consequence, the model for the amorphous state for carburized and nitrided samples can not be applied in the same form for oxidized samples.

According to He et al. [118] and Kodama et al. [135], $\mathrm{NiFe}_{2} \mathrm{O}_{4}$ cores surrounded by spin-glassy surface shells satisfy the observed results. In contrast to the nitrided quasi metallic phase, for the oxidized phase a more exact investigation (e.g. EXAFS) is required to resolve the microstructure.

Magnetron sputtering is suitable to form amorphous and quasi metallic glasses, but the stoichiometric FeC phase was not obtained. As a consequence, iron/carbon and for comparison stainless steel/carbon films were deposited by PLD. The re- 


\section{Chapter 7}

\section{Results for pulsed laser deposited films}

\author{
Results using the Siemens XP2020 ex- \\ cimer laser
}

\subsection{Results for FeC films pre- pared by STPLD}

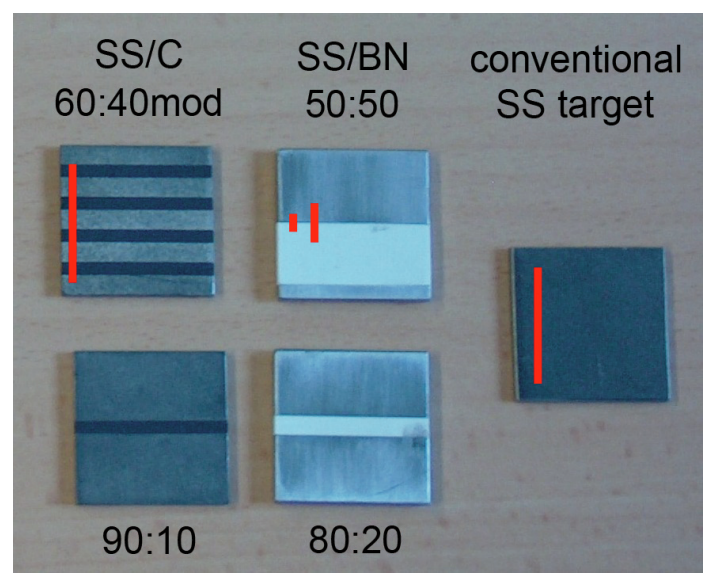

Figure 7.1: Different stainless steel STPLD targets in comparison to a conventional stainless steel PLD target (right). The red lines indicate the laser scan area. As indicated in SS/BN 50:50 STPLD target, for ultra-thin films a scan area of $4 \mathrm{~mm}(4 \mathrm{~mm}$ stainless steel $/ 4 \mathrm{~mm} \mathrm{C}$ ) and $8 \mathrm{~mm}(8 \mathrm{~mm}$ stainless steel $/ 8 \mathrm{~mm} \mathrm{C}$ ) for thin-films were used.

Ultra-thin high-carbon iron films (ARMCO, $99.96 \%$; maximal thickness $20 \mathrm{~nm}$ ) were deposited by Sequential Target Pulsed Laser Deposition (STPLD) with a commercial Siemens XP2020 excimer laser $(\lambda=308 \mathrm{~nm}$, pulse duration $55 \mathrm{~ns}$, repetition rate $8 \mathrm{~Hz}$ ) on TEM grids in order to directly examine the structure of the STPLD films. The STPLD process relies on the pre-combination of the target to a desired stoichiometry (assuming the same ablation rate for all elements) as shown in Fig. 7.1 and is determined by the inlay thickness and the laser scan area.

For comparison films with higher thicknesses were deposit onto amorphous $\mathrm{SiO}_{2}$ substrates (oxidized $\mathrm{Si}(100)$ wafer of $0.5 \mathrm{~mm}$ thickness, pre-cleaned with acetone and oxidized in air, no further treatment) and the influence of substrate temperature was investigated. The deposition parameters and the resulting thicknesses and growth rates are summarized in Table 7.1.

Table 7.1: Deposition temperature $\mathrm{T}_{d e p}$, laser energy, number of pulses \#, real film thickness $d$ (as measured by Rutherford Backscattering Spectrometry (RBS) after deposition) and derived growth rate $g=\frac{d}{t}$ for the deposited STPLD films.

\begin{tabular}{cccccr}
\hline sample & $\begin{array}{c}\mathrm{T}_{\text {dep }} \\
{[\mathrm{K}]}\end{array}$ & $\begin{array}{c}\text { energy } \\
{\left[\mathrm{mJ} / \mathrm{cm}^{2}\right]}\end{array}$ & $\#$ & $\begin{array}{c}d \\
{[\mathrm{~nm}]}\end{array}$ & $\begin{array}{r}g \\
{[\mathrm{~nm} / \mathrm{min}]}\end{array}$ \\
\hline $\mathrm{FeC} 4 \mathrm{~mm}$ & 298 & 1.30 & 933 & $20(5)$ & $10.26(87)$ \\
$\mathrm{FeC} 8 \mathrm{~mm}$ & 298 & 0.96 & 36000 & $77(10)$ & $1.03(9)$ \\
$\mathrm{FeC} 8 \mathrm{~mm}$ & 823 & 1.00 & 43000 & $258(10)$ & $2.88(30)$ \\
\hline
\end{tabular}

1 this thickness was derived from a quartz microbalance during deposition

Synthesis of NaCl-type FeC The directly TEM-prepared STPLD showed different phases, inter alia many face-centered cubic structures as seen in Fig 7.2.

By taking the diffraction pattern of one of the grains, it could be identified as $\gamma$-Fe.

One characteristic was found in all the samples: all the grains were embedded in an amorphous $\mathrm{Fe}$ matrix. Another astonishing feature can be seen in Fig. 7.3. Some grains were surrounded by $\mathrm{Fe}$ and C multilayers with a thickness of $0.238(4) \mathrm{nm}$ which merge fluently into the grains. This could be a hint that stress is a crucial factor for phase formation in these thin films. On the right corner of Fig. 7.3, the FFT can be seen. It shows the appearance of a metallic glass which indicates a high-carbon concentration.

This grain has a diameter of about $10 \mathrm{~nm}$. All 


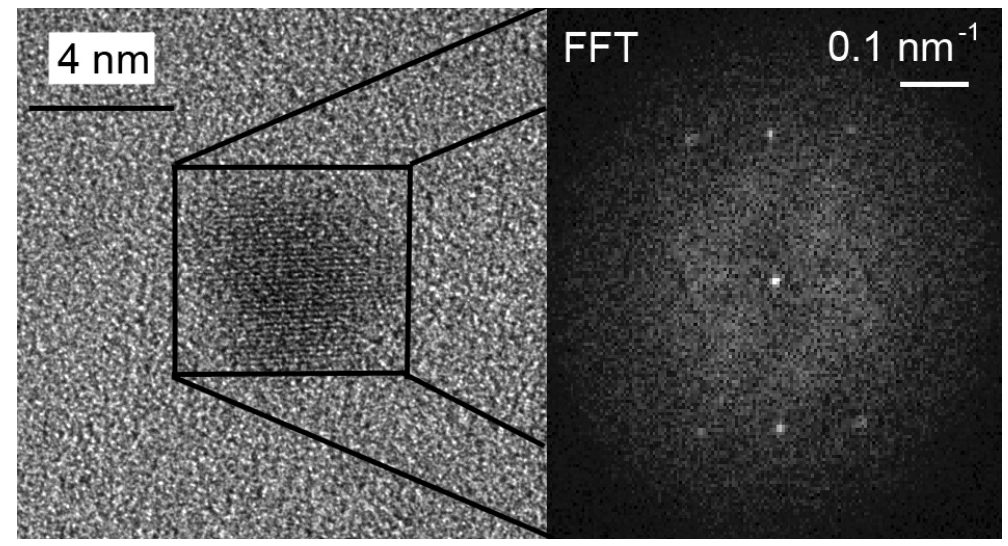

Figure 7.4: TEM pattern of the NaCl-type FeC grain and its FFT on the right hand.

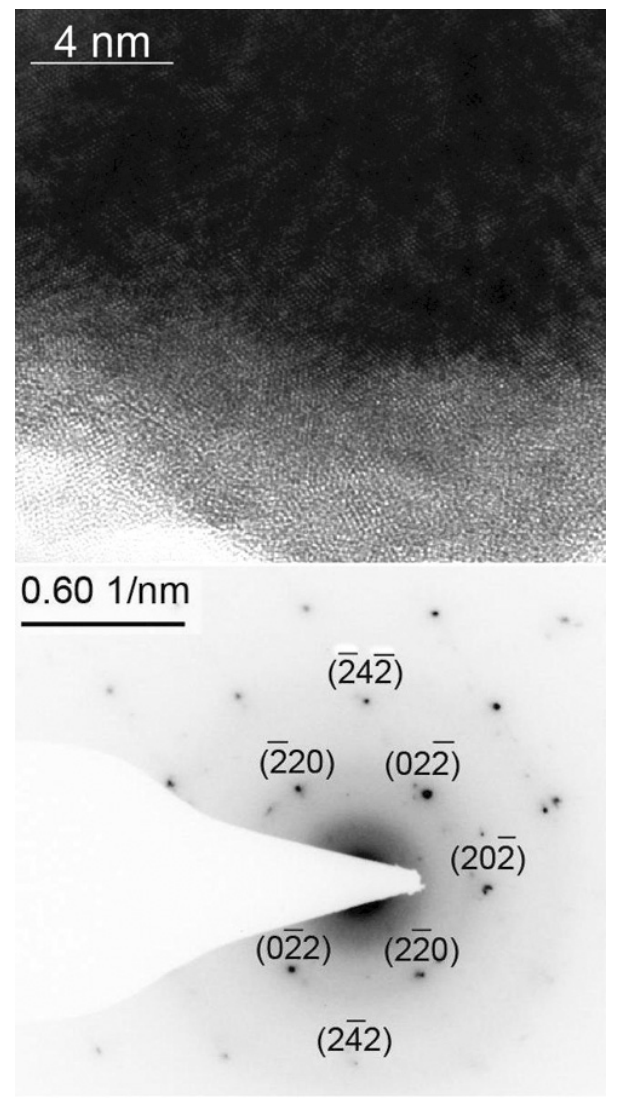

Figure 7.2: (top) HR-TEM pattern of a grain. (bottom) diffraction pattern of this grain. The orientation of the reflexes are given in the graph.

attempts to obtain an SAD pattern from these grains were futile, because the grains disappeared after focussing the electron beam. These grains seem to be thermally unstable. In order to get information on the structure a FFT was taken, which is depicted on the right side of Fig. 7.4. Fig. 7.4 shows one of the various nano-scale grains

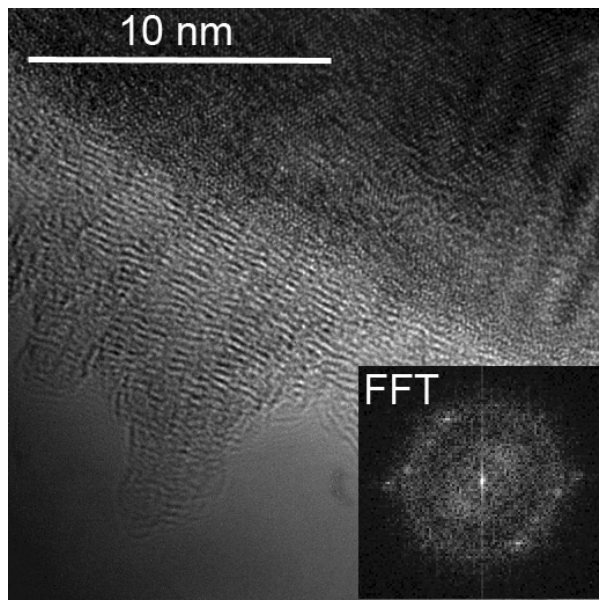

Figure 7.3: Fine Fe and $\mathrm{C}$ multilayer system surrounding a metallic glassy grain observed by TEM and its FFT of that area (right).

which could be observed over the whole film. Lee et al. [9] predicted in the NaCl-type FeC phase a bond length of $0.236 \mathrm{~nm}$, the value obtained here is $0.229(3) \mathrm{nm}$. As a consequence, the obtained lattice constant $a_{0}=0.458(6) \mathrm{nm}$ is verisimilar to the predicted $\mathrm{NaCl}$-type lattice constant $a_{0}=0.473 \mathrm{~nm}$. EDX does not show any hints of other element impurities, only $\mathrm{Fe}$ and $\mathrm{C}$ are observed. As known from the $\mathrm{Fe}-\mathrm{C}$ system so far, no simple cubic structure $\mathrm{Fe}-\mathrm{C}$ exists, but the observed structure seems to prove the predicted $\mathrm{NaCl}$-type $\mathrm{Fe}-\mathrm{C}$.

As next step, films with higher thicknesses were deposit to investigate the macroscopical properties of this phase.

Fig. 7.5 shows the XRD pattern of the STPLD sample sputtered at 298 and $823 \mathrm{~K}$. 


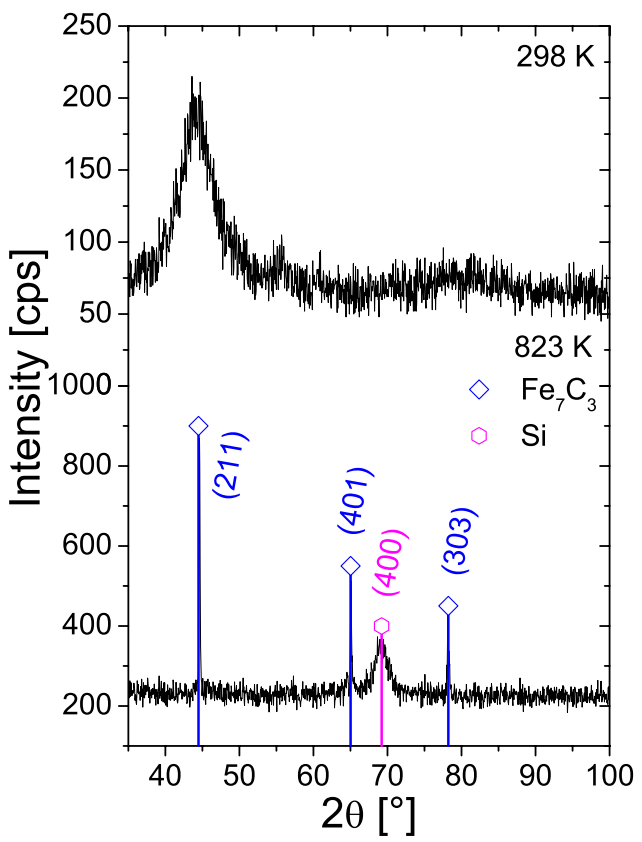

Figure 7.5: GIXRD $\left(2^{\circ}\right)$ spectra of the STPLD films deposited at 298 (top) and $823 \mathrm{~K}$ (bottom). The temperatures and reflexes are given in the graph.

Deposition of NaCl-type FeC films The spectrum for the STPLD films sputtered at $298 \mathrm{~K}$ show the typical broad XRD appearance of amorphous materials. A mean grain size of $3(2) \mathrm{nm}$ can be calculated with the Scherrer formula [139]:

$$
\Delta \beta=\frac{0.89 \lambda}{d \cdot \cos \theta}
$$

In contrast, the STPLD film deposited at $823 \mathrm{~K}$ exhibit clear reflexes, which can be attributed to the $\mathrm{Fe}_{7} \mathrm{C}_{3}$-phase [130]. It is well crystallized and a mean grain size of $50(5) \mathrm{nm}$ is derived by eq. 7.1 [139].

Fig. 7.6 summarizes the CEMS measurements of the samples deposited at $298 \mathrm{~K}$ and $823 \mathrm{~K}$. For these spectra, hyperfine field and quadrupole splitting distributions were assumed, respectively. The quadrupole splitting distributions are broad and close to the values of $\mathrm{Fe}_{3} \mathrm{C} / \mathrm{Fe}_{7} \mathrm{C}_{3}$ carbides (which is confirmed by a Lorentzian single line analysis). This is in good agreement with the XRD pattern for the STPLD sample deposited at $823 \mathrm{~K}$, which clearly exhibit the $\mathrm{Fe}_{7} \mathrm{C}_{3}$-phase. The distribution for the sample deposited at $298 \mathrm{~K}$ is broader than that deposited at $823 \mathrm{~K}$. Taking the XRD pattern into account, the CEM spectrum could be attributed to an amorphous FeC alloy. Both samples show magnetic split sextets, however, the hyperfine fields of both films are diminished. This can be attributed to carbon which should be in the next-nearest neighborhood. By using the
Table 7.3: Hyperfine interaction parameters of the singe line analysis of the STPLD FeC film deposited at $823 \mathrm{~K}$. Hyperfine parameters: $\delta$ is the isomer shift, QS the quadrupole splitting, $\Gamma$ the line width (HWHM), and RA the relative area.

\begin{tabular}{cccc}
\hline$\delta$ & QS & $\Gamma$ & RA \\
{$[\mathrm{mm} / \mathrm{s}]$} & {$[\mathrm{mm} / \mathrm{s}]$} & {$[\mathrm{mm} / \mathrm{s}]$} & {$[\%]$} \\
\hline $0.15(7)$ & $0.49(22)$ & 0.25 & $47.9(30)$ \\
$0.17(11)$ & $0.71(37)$ & 0.25 & $52.1(21)$ \\
\hline
\end{tabular}

dependency between median hyperfine field and carbon content for carburized stainless steel films as found in 6.2.7 and by inserting the hyperfine field of ARMCO-iron, a linear correlation between hyperfine field and carbon content is found (Fig. 7.7):

$$
y=-0.38(3) x+30.32(164) \mathrm{T}
$$

Note: due to the fact, that $\mathrm{Ni}$ and $\mathrm{Cr}$ have a crucial

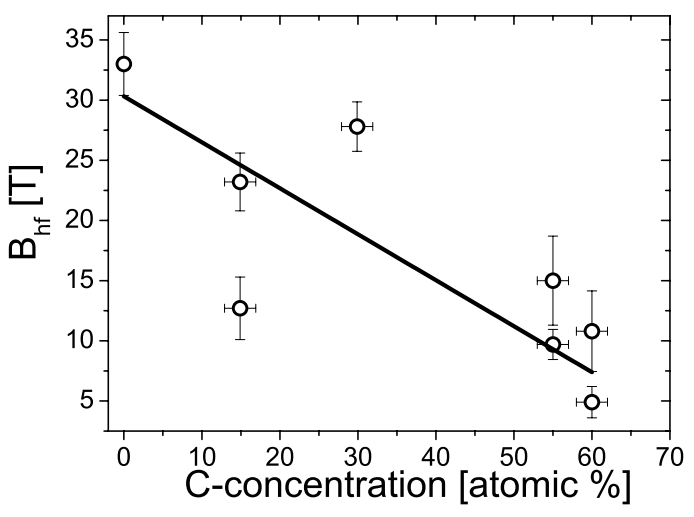

Figure 7.7: Median hyperfine field $\mathrm{B}_{\mathrm{hf}}$ in dependence of the carbon content.

influence on the hyperfine field, the slope for $\mathrm{FeC}$ films should be smaller; thus the carbon content in FeC films should be greater than predicted by this correltation.

Consequently, a carbon content of about 9.0(12) \% and $36.8(22) \%$ is predicted for the samples deposited at $298 \mathrm{~K}$ and $823 \mathrm{~K}$, respectively.

The fitted hyperfine parameters are presented in Table 7.2, the results of the single line analysis in Table 7.3 .

While Mössbauer Spectroscopy revealed hyperfine fields, MOKE did not exhibit any signal, which can be attributed to the different information depths of both methods (MOKE is surface sensitive).

To investigate the carbon content in the films, RBS measurements were carried out, which can be seen in Fig. 7.8.

The results of the RBS analysis are summarized in Table 7.4 . 

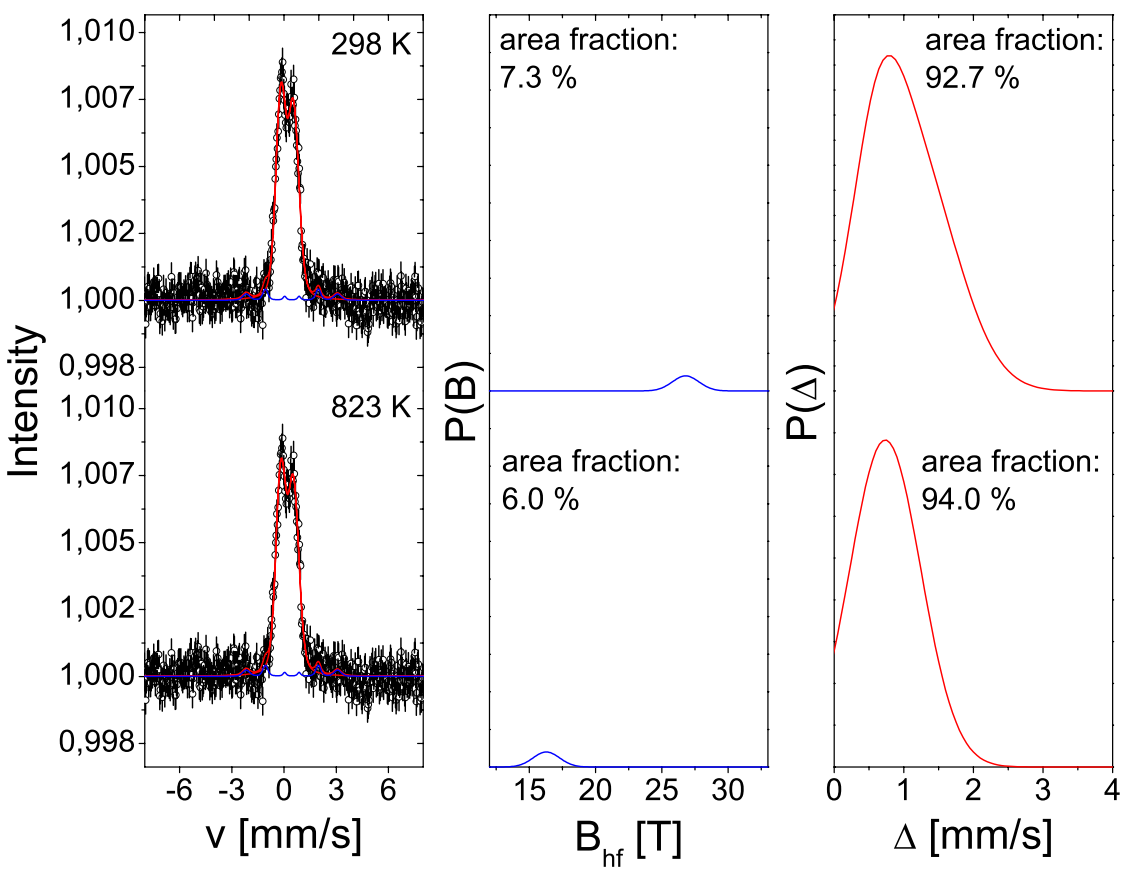

Figure 7.6: Mössbauer spectra of the STPLD deposited FeC films at $298 \mathrm{~K}$ (top) and $823 \mathrm{~K}$ (bottom).

Table 7.2: Mössbauer fitting results of the STPLD deposited FeC films: $(f$ - area fraction (error), mean values of $\langle\delta\rangle$ - isomer shift, $\langle\Delta\rangle$ - the quadrupole splitting for the paramagnetic subspectra, $\langle\epsilon\rangle$ the quadrupole splitting for the magnetic subspectra, $B$ - hyperfine field).

\begin{tabular}{ccrrrrrr}
\hline $\begin{array}{c}\text { FeC } \\
\text { sample }\end{array}$ & part & $\begin{array}{r}f \\
{[\%]}\end{array}$ & $\begin{array}{r}\langle\delta\rangle \\
{[\mathrm{mm} / \mathrm{s}]}\end{array}$ & $\begin{array}{r}\langle\Delta\rangle,\langle\epsilon\rangle \\
{[\mathrm{mm} / \mathrm{s}]}\end{array}$ & $\begin{array}{r}\sigma_{\Delta} \\
{[\mathrm{mm} / \mathrm{s}]}\end{array}$ & $\begin{array}{r}\langle B\rangle \\
{[\mathrm{T}]}\end{array}$ & $\begin{array}{r}\sigma_{B} \\
{[\mathrm{~T}]}\end{array}$ \\
\hline $298 \mathrm{~K}$ & ferro & $7.3(5)$ & 0.25 & -0.04 & & $26.9(29)$ & $1.0(5)$ \\
& para-1 & $4.8(9)$ & 0.21 & 0.75 & 0.12 & & \\
& para-2 & $87.9(43)$ & & 0.97 & 0.66 & & \\
\hline $823 \mathrm{~K}$ & ferro & $6.0(5)$ & 0.45 & & & $16.3(15)$ & $1.0(5)$ \\
& para & $94.0(2)$ & 0.18 & 0.73 & 0.51 & & \\
\hline
\end{tabular}

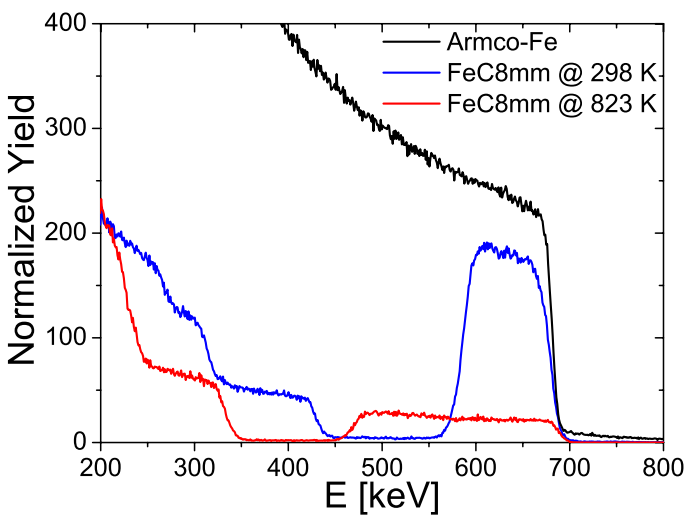

Figure 7.8: RBS spectra of the STPLD films deposited at 298 and $823 \mathrm{~K}$.

As obtained from the RBS analysis, the carbon contents are in good agreement as derived from the Mössbauer valuation. However, STPLD films
Table 7.4: Results of the RBS analysis of the STPLD deposited films.

\begin{tabular}{cccc}
\hline sample & $\begin{array}{c}\mathrm{Fe} \\
\text { at. } \%\end{array}$ & $\begin{array}{c}\mathrm{C} \\
\text { at. } \%\end{array}$ & $\begin{array}{c}\mathrm{O} \\
\text { at. } \%\end{array}$ \\
\hline $298 \mathrm{~K}$ & $80(5)$ & $10(5)$ & $10(5)$ \\
$823 \mathrm{~K}$ & $10(5)$ & $40(5)$ & $50(5)$ \\
\hline
\end{tabular}

and Magnetron-sputtered FeC films have the additional oxygen content in common. According to Huczkowski [140], the small addition of $\mathrm{Si}$ in Febased alloys enhances the oxide formation. Thus, the inter-diffusion between $\mathrm{Fe}$ and the substrate $\mathrm{SiO}_{2}$ (as obtained by the RBS depth-profile) favours the formation of oxides in the film. Conspicous is, that neither Mössbauer Spectroscopy nor XRD show any hints of oxide formation. This yields a conclusion that only parts of the oxygen content participate on oxide formation. The remaining part is arranging at the grain boundaries.

Comparing theproperties of high-carbon films, 
which were directly deposited on TEM grids, with thicker ones deposited on $\mathrm{SiO}_{2}$ substrates, the assumption arises that the observed $\gamma$ - and $\mathrm{NaCl}$-type structure could be due to a thickness effect.

Indeed, thickness effects are observed in thin Fe and stainless steel films, wherein the formation of thin $\gamma$-layers $(5-10 \mathrm{~nm})$ are thermodynamically favored [141].

Summarizing, the synthetization of NaCl-type $\mathrm{FeC}$ succeded in ultra-thin films $(\sim 20 \mathrm{~nm})$. More investigations have to be done to synthesize thin $\mathrm{NaCl}-$ type $\mathrm{FeC}$ films (e.g. chosse different substrates, apllying biases at the substrates etc.).

As well as for Magnetron-sputtered FeC films, the STPLD film deposit at $298 \mathrm{~K}$ exhibit an amorphous signature. Therefore, stainless steel films prepared by Reactive Pulsed Laser Deposition (RPLD) were deposited to compare those with Magnetron sputtered films and to possible obtain the new amorphous and soft ferromagnetic disordered $\mathrm{Ni}_{3} \mathrm{C}$ phase.

\subsection{Results for carburized stainless steel films pre- pared by RPLD}

The film was reactive sputter-deposited by pulsed laser deposition (RPLD) with a Siemens XP2020 excimer laser $(\lambda=308 \mathrm{~nm}$, pulse duration $55 \mathrm{~ns}$, repetition rate $8 \mathrm{~Hz}$ ) onto amorphous $\mathrm{SiO}_{2}$ substrates (oxidized $\mathrm{Si}(100)$ wafer of $0.5 \mathrm{~mm}$ thickness, pre-cleaned with acetone and oxidized in air, no further treatment) utilizing a commercial AISI 316 (X5CrNiMo17-12-2, 1.4401) target. Before deposition, the PLD-chamber was evacuated to a base pressure of $10^{-6} \mathrm{~Pa}$ to prevent hysteresis effects. The laser energy was set to $2 \mathrm{~mJ} / \mathrm{cm}^{2}$. The $\mathrm{CH}_{4}$ gas was tuned by the vacuum penning and set to a total chamber pressure of $10^{-5} \mathrm{~Pa}$. The growth rate $g=\frac{d}{t}$ was derived to $0.96(4) \mathrm{nm} / \mathrm{min}$ by using the thickness $d=80 \mathrm{~nm}$ as measured by RBS after deposition.

\subsubsection{Deposition at room temper- ature and influence of the methane gas flow}

In contrast to the original AISI 316 target as illustrated in Fig. 5.1), which shows the typical $\gamma$ structure $(a=0.3592(1) \mathrm{nm})$, the RPLD sample (illustrated in Fig. 7.9) shows both $\gamma-(\mathrm{Fe}, \mathrm{Cr}, \mathrm{Ni})$ and $\alpha$ - $(\mathrm{Fe}, \mathrm{Cr}, \mathrm{Ni})$ structures, but a small broad signature is observed as well indicating an amorphous phase.

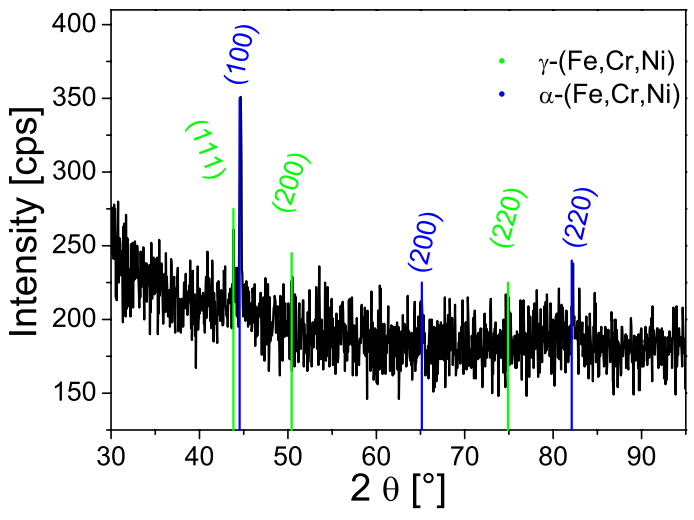

Figure 7.9: GIXRD $\left(2^{\circ}\right)$ spectra of the RPLD film. The reflexes are given in the graph.

The lattice constant were derived to.

In contrast to the XRD pattern, the CEM spectrum is depicted in Fig. 7.10.

It reveals the typical Mössbauer spectrum of an amorphous material and is magnetically split. The results of the fitting procedure are presented in Table 7.5 .

As well as carburized stainless steel films prepared Magnetron sputtering, the Mössbauer spectra were fitted by hyperfine field distributions, and with quadrupole splitting distributions in the paramagnetic state, respectively. The distributions are attributed to an amorphous stainless steel carbonalloy, possibly rich in carbon. In contrast to carburized stainless steel films, the ratio between high-field and low-field amorphous state are different. Here, the high field distribution dominates. From the fitting, a hyperfine field of $B=15.6 \mathrm{~T}$ with a width of $\sigma=8.5 \mathrm{~T}$ is obtained from the $p(B)$ distribution. The high $\sigma$ is indicating a participation of other hyperfine fields, which could possibly correspond to an $\alpha$-phase, but there is no clear indicator for this (in contrast to the XRD pattern). Now following question arise: where does the CEM spectrum differ from the XRD pattern?

The answer can be seen in Fig. 6.33: the dark field image (TEM) of the disordered $\mathrm{Ni}_{3} \mathrm{C}$ phase shows many crystallites at the interface between film and $\mathrm{SiO}_{2}$ substrate (the first $50 \mathrm{~nm}$ ) embedded in an amorphous matrix. Since the overall thickness of the layer is derived to $80 \mathrm{~nm}$ as measured by RBS, the differing observations from Mössbauer and XRD can be explained by the differing information depth of both methods.

CEMS is sensitive to the first $150 \mathrm{~nm}$, whereas the GIXRD with $\omega=2^{\circ}$ corresponds to an information depth of $350 \mathrm{~nm}$. Additionally, only the reflexes are shown in GIXRD, which have the correct orientation. As a consequence, the fraction of the 

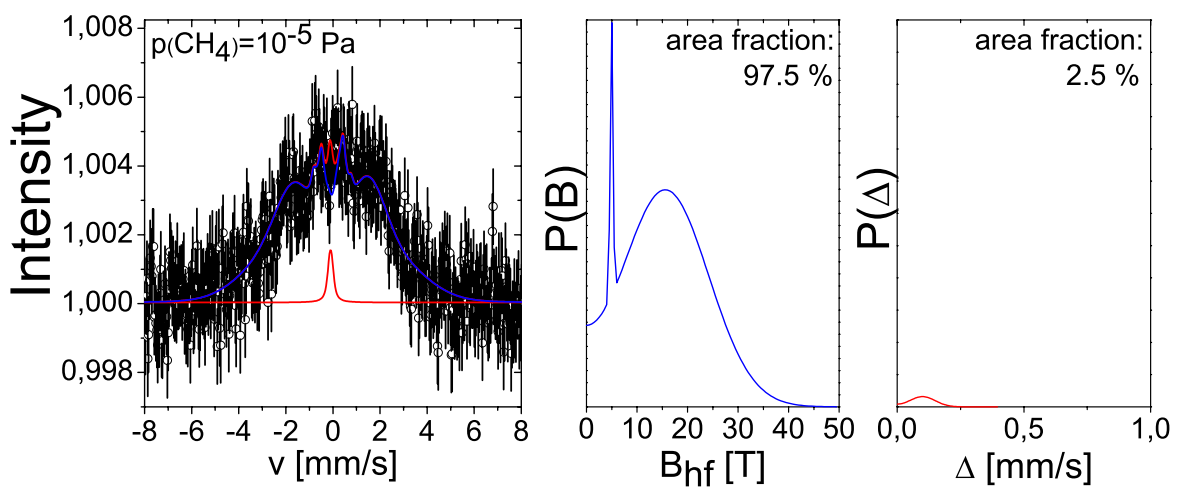

Figure 7.10: CEM spectrum of the RPLD sample. The $\mathrm{CH}_{4} /$ chamber pressure is given in the graph.

Table 7.5: Mössbauer fitting results of the RPLD film, deposited at room temperature: $(f$ - area fraction (error), mean values of $\langle\delta\rangle$ - isomer shift, $\langle\Delta\rangle$ - the quadrupole splitting for the paramagnetic subspectra, $\langle\epsilon\rangle$ the quadrupole splitting for the magnetic subspectra, $B$ - hyperfine field).

\begin{tabular}{ccrrrrrr}
\hline $\begin{array}{c}\mathrm{CH}_{4} \\
\text { pressure } \\
{[\mathrm{Pa}]}\end{array}$ & part & $f$ & $\langle\delta\rangle$ & $\langle\Delta\rangle,\langle\epsilon\rangle$ & $\sigma_{\Delta}$ & $\langle B\rangle$ & $\sigma_{B}$ \\
\hline \multirow{2}{*}{$10^{-6}$} & ferro-l & $4.1(58)$ & {$[\mathrm{mm} / \mathrm{s}]$} & {$[\mathrm{mm} / \mathrm{s}]$} & {$[\mathrm{mm} / \mathrm{s}]$} & {$[\mathrm{T}]$} & {$[\mathrm{T}]$} \\
& ferro-h & $93.4(74)$ & -0.04 & 0.02 & & $5.0(12)$ & $0.3(12)$ \\
& para & $2.5(45)$ & -0.10 & 0.02 & & $15.6(12)$ & $8.5(15)$ \\
\hline
\end{tabular}

amorphous phase could be very small in comparison to the crystallites. In contrast, CEMS is sensitive to the whole film and thus the volume fraction of amorphous phase and crystallites are different as observed in GIXRD. As a consequence, the CEM spectrum is dominated by the spectrum of the amorphous phase.

Carbon content was derived by RBS measure-

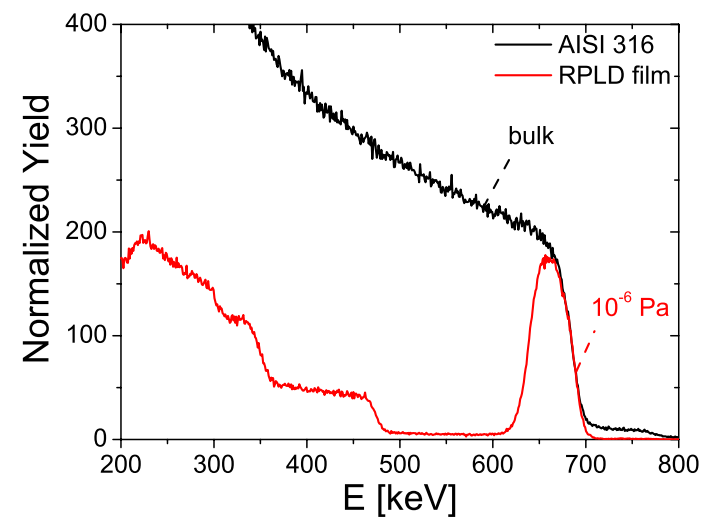

Figure 7.11: RBS spectra of the RPLD films. The $\mathrm{CH}_{4}$ /chamber pressure is given in the graph.

ments, which are depicted in Fig. 7.11.

A carbon content of $14(2) \%$ is found, which is about $45 \%$ less than in carburized stainless steel films prepared by Magnetron-sputtering [7,91]. Another fea- ture is the decrease of the Mo content (under $1 \%$ ), whereas the Ni content is simultaneously increasing.

\subsubsection{Modification of the nucleation model}

This could be another confirmation of the nucleation model presented in 6.2.6: as mentioned above, this sample shows the beginning of the formation of the disordered $\mathrm{Ni}_{3} \mathrm{C}$ phase. During deposition the Mo atoms insert long range diffusion and are hindering phase, but not completely. Inhomogeneities in the inserted long range diffusion allow the formation of nano-crystallites, while the carbon is solving into the matrix. In this stage of nucleation, the Mo atoms were re-sputtered, which leads to a diminution of Mo in the film of under $1 \%$ and $\mathrm{Ni}$ content increases.

At a critical carbon content of 10-15\%, the metastable - but disordered - $\mathrm{Ni}_{3} \mathrm{C}$ phase is formed and the carbon diffusion is fully established (inserted by the Mo atoms at the beginning of the deposition process and which are additionally hindering the nucleation process by establishing higher atomic mismatches through higher probabilities of coordination). As a consequence, the Mo content rises again to the target stoichiometry. For films with thicknesses above $250 \mathrm{~nm}$ (like those deposited in 6.2), the Mo decrease in the first $30-50 \mathrm{~nm}$ is not 
commensurable by EDX.

In the course of deposition, more and more carbon is adsorbed, which leads to the formation of graphite and spin-glassy phases. Thus, for films with higher thicknesses excessive $\mathrm{C}$ contents are observed, but the critical carbon content seems to be between 10 and $15 \%$, which is also seen for nitrided samples (see 6.3 and [134]).

Excessive carbon content can be prevented by higher laser densities. As a consequence, a Quantel Brilliant Nd:YAG laser is used for further experiments (its laser density is 4 times higher than that of the used excimer laser). The results are shown in the following sections.

\section{Results using the Quantel Brilliant Nd:YAG laser}

\subsection{Results for carburized stainless steel films pre- pared by RPLD}

The films were reactive sputter-deposited by pulsed laser deposition (RPLD) with a Brilliant Nd:YAG laser $(\lambda=1064 \mathrm{~nm}$, pulse duration 5-6 ns, repetition rate $20 \mathrm{~Hz}$ ) onto amorphous $\mathrm{SiO}_{2}$ substrates (oxidized $\mathrm{Si}(100)$ wafer of $0.5 \mathrm{~mm}$ thickness, precleaned with acetone and oxidized in air, no further treatment) utilizing a commercial AISI 316 (X5CrNiMo17-12-2, 1.4401) target. Before deposition, the PLD-chamber was evacuated to a base pressure of $10^{-6} \mathrm{~Pa}$ to prevent hysteresis effects. The laser energy was set to $5 \mathrm{~mJ} / \mathrm{cm}^{2}$. The $\mathrm{CH}_{4}$ gas was tuned by the vacuum penning. Thus, total chamber pressures of $5 \cdot 10^{-5}-1 \cdot 10^{-1} \mathrm{~Pa}$ were generated. The thickness of the deposited films was controlled by a quartz microbalance (with a film density set to $7.89 \mathrm{~kg} / \mathrm{m}^{3}$, which is the density of AISI 316). The deposition parameters and the resulting thicknesses and growth rates are summarized in Table 7.6.

From these data it is derived that the growth rate has a parabolic form with increasing $\mathrm{CH}_{4}$ /chamber pressure, which is visualized in Fig. 7.12.

As demonstrated by Mössbauer Spectroscopy, the maximum of this curve indicates the transition to the amorphous and soft ferromagnetic disordered $\mathrm{Ni}_{3} \mathrm{C}$ phase.
Table 7.6: Total chamber pressure $j$, deposition time $t$, real film thickness $d$ (as measured by RBS after deposition) and derived growth rate $g=\frac{d}{t}$ for the carburized RPLD films. All samples were deposited at $298 \mathrm{~K}$ with a laser energy of $5 \mathrm{~mJ} / \mathrm{cm}^{2}$ and a target-substrate distance of $7.5 \mathrm{~cm}$.

\begin{tabular}{ccccc}
\hline sample & $\begin{array}{c}j \\
{[\mathrm{~Pa}]}\end{array}$ & $\begin{array}{c}t \\
{[\mathrm{~min}]}\end{array}$ & $\begin{array}{c}d \\
{[\mathrm{~nm}]}\end{array}$ & $\begin{array}{c}g \\
{[\mathrm{~nm} / \mathrm{min}]}\end{array}$ \\
\hline $\mathrm{Nd} 5-5$ & $5 \cdot 10^{-5}$ & $29: 58$ & $130(10)$ & $4.33(37)$ \\
$\mathrm{Nd} 1-4$ & $1 \cdot 10^{-4}$ & $34: 35$ & $162(10)$ & $4.68(32)$ \\
$\mathrm{Nd} 5-4$ & $5 \cdot 10^{-4}$ & $31: 11$ & $243(10)$ & $7.79(41)$ \\
$\mathrm{Nd} 1-3$ & $1 \cdot 10^{-3}$ & $31: 32$ & $172(10)$ & $5.46(36)$ \\
$\mathrm{Nd} 1-2$ & $1 \cdot 10^{-2}$ & $21: 29$ & $272(10)$ & $12.66(77)$ \\
$\mathrm{Nd} 1-1$ & $1 \cdot 10^{-1}$ & $21: 34$ & $85(10)$ & $3.94(50)$ \\
\hline
\end{tabular}

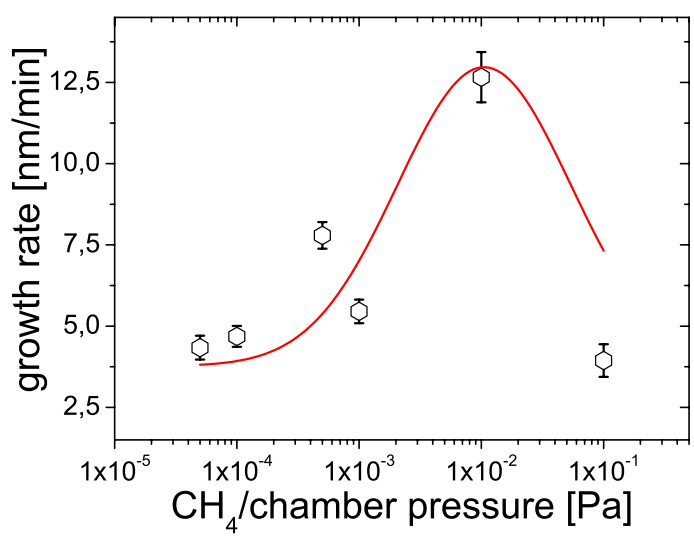

Figure 7.12: Growth rate $g$ as a function of the $\mathrm{CH}_{4} /$ chamber pressure for the room temperature deposition.

\subsubsection{Deposition at room temper- ature and influence of the methane gas flow}

The methane/chamber pressure was systematically increased from $5 \cdot 10^{-5}-1 \cdot 10^{-1} \mathrm{~Pa}$. For all these deposited films, the reactive pulsed laser deposited samples show the typical broad XRD appearance of amorphous materials; additionally Fig. 7.13 c), e) and f) show appendages of crystalization. The GIXRD patterns are shown in Fig. 7.13.

Fig. 7.14 shows the position and the width of the first broad peak for the spectra shown in Fig. 7.13. There is a clear tendency for peak position and peak width, where the peak position is decreasing with increasing $\mathrm{CH}_{4} /$ chamber pressure, excerpt the RPLD sample deposited at $10^{-3} \mathrm{~Pa}$.

Fig. 6.19 summarizes the CEMS measurements of the samples with $\mathrm{CH}_{4} /$ chamber pressure.

They show the typical Mössbauer spectra of amorphous materials. All spectra are magnetically split, even if the hyperfine field in spectrum b) is small. It seems, that the disordered phase is also here formed, 

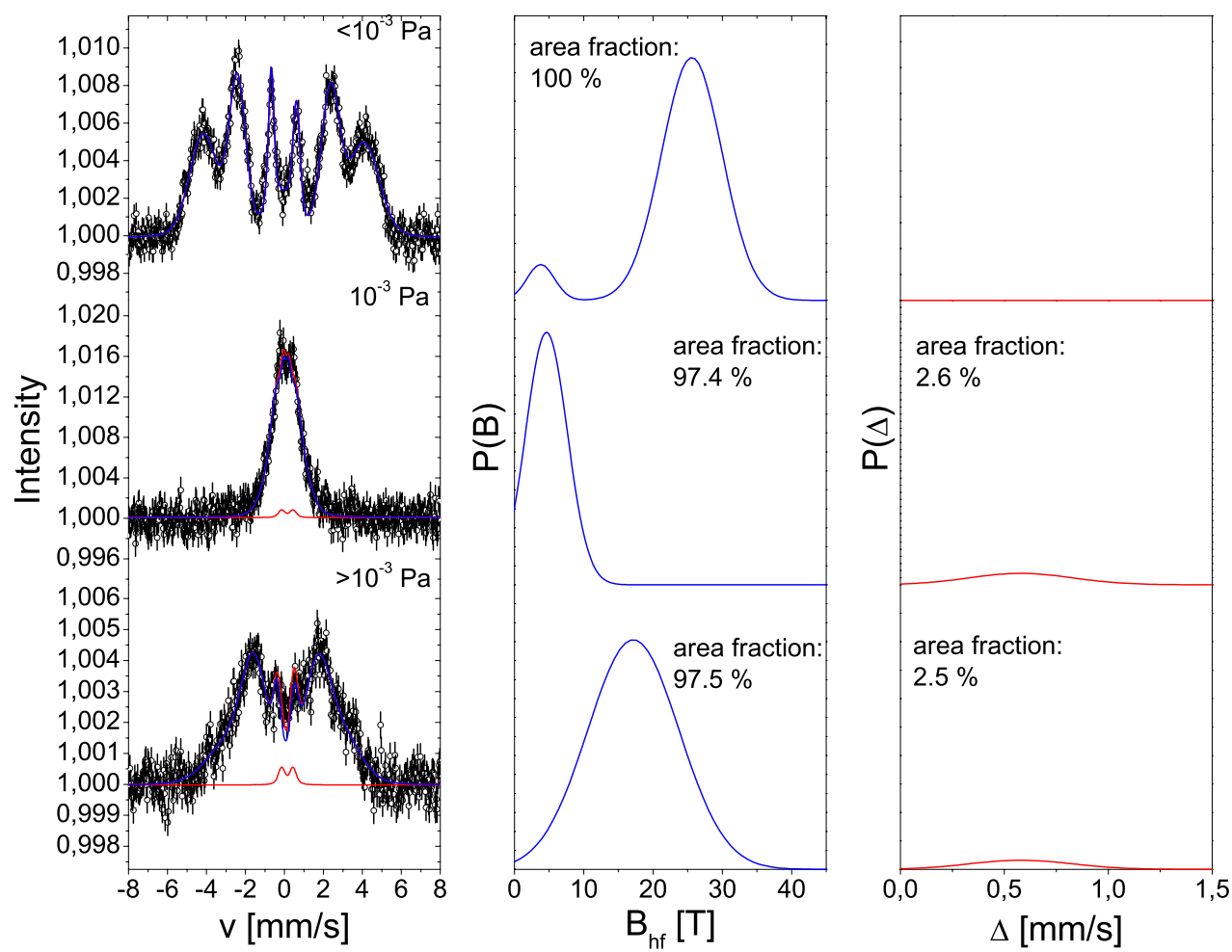

Figure 7.15: Mössbauer spectra of the reactive sputtered AISI 316 films. The numbers in the graphs represent the methane gas flow.

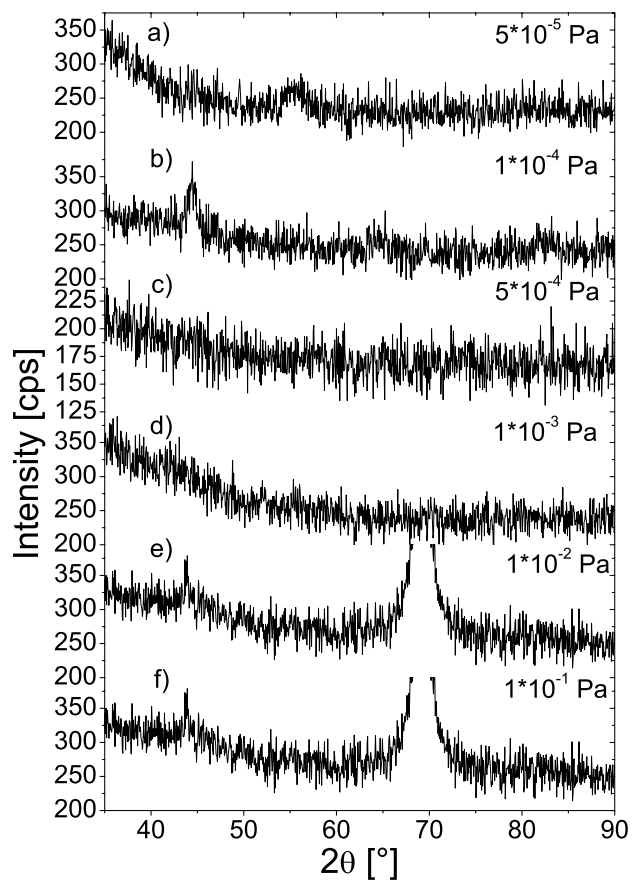

Figure 7.13: GIXRD $\left(2^{\circ}\right)$ spectra of the carburized RPLD films. The $\mathrm{CH}_{4} /$ chamber pressure are given in the graph.

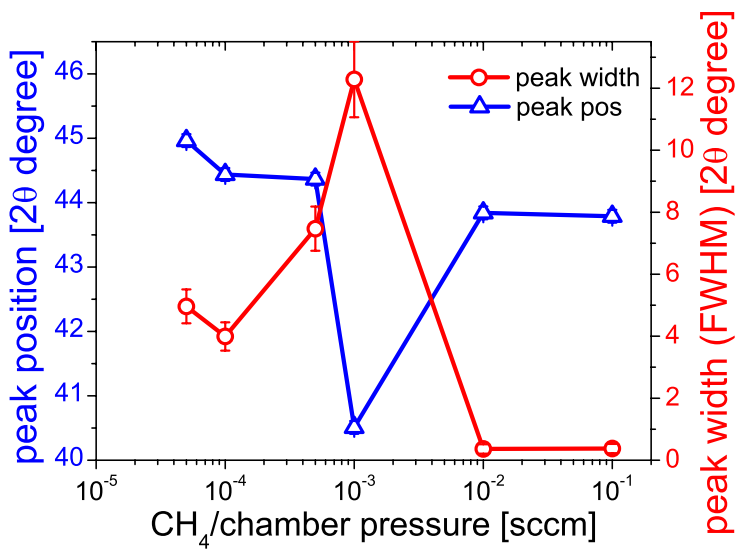

Figure 7.14: Peak position and peak width of the first broad peak in the XRD spectra of the RPLD stainless steel films.

but the screw thread of the gas inlet was not fine enough to observe the full transition. The results of the fitting procedures are presented in Table 7.7. The Mössbauer results were confirmed by MOKE measurements. They show the same behavior as for the spectra presented in 6.2.1.

The EDX analyses of the deposited films revealed the original composition of the sputtering targets 
Table 7.7: Mössbauer fitting results of the RPLD films, deposited at room temperature with a laser energy of $5 \mathrm{~J} / \mathrm{cm}^{2}$ ( $f$ - area fraction (error), mean values of $\langle\delta\rangle$ - isomer shift, $\langle\Delta\rangle$ - the quadrupole splitting for the paramagnetic subspectra, $\langle\epsilon\rangle$ the quadrupole splitting for the magnetic subspectra, $B$ hyperfine field).

\begin{tabular}{ccrrrrrr}
\hline $\begin{array}{c}\mathrm{CH}_{4} \\
\text { pressure } \\
{[\mathrm{Pa}]}\end{array}$ & part & $f$ & $\langle\delta\rangle$ & $\langle\Delta\rangle,\langle\epsilon\rangle$ & $\sigma_{\Delta}$ & $\langle B\rangle$ & $\sigma_{B}$ \\
\hline$<10^{-3}$ & ferro-l & $6.3(12)$ & -0.04 & -0.01 & & $3.8(7)$ & $2.0(9)$ \\
& ferro-h & $93.7(55)$ & -0.04 & -0.01 & & $25.6(2)$ & $1.3(7)$ \\
\hline $10^{-3}$ & ferro & $97.4(22)$ & -0.03 & -0.05 & & $4.8(25)$ & $2.7(38)$ \\
& para & $2.6(5)$ & 0.15 & 0.58 & 0.24 & & \\
\hline$>10^{-3}$ & ferro & $97.5(12)$ & 0.04 & & & $17.2(5)$ & $6.6(4)$ \\
& para & $2.5(3)$ & 0.15 & 0.58 & 0.24 & & \\
\hline
\end{tabular}

within the experimental limits. Unfortunately, the EDX did not allow to accurately determine elements lighter than oxygen. Therefore, in order to evaluate the amount of incorporated carbon, RBS measurements were carried out, whose results are shown in Fig. 7.16.

The thickness of the films as obtained from the

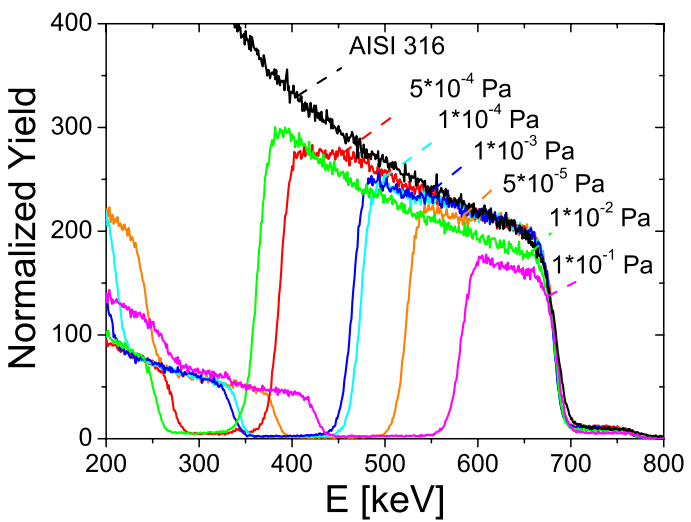

Figure 7.16: RBS spectra of the RPLD films. The $\mathrm{CH}_{4}$ /chamber pressure is given in the graph.

RBS analysis was already given in Table 7.6. The carbon concentration of the films as obtained from the RBS analysis is given in Fig. 7.17.

A clear tendency can be seen, where the $\mathrm{C}$ content increases with $\mathrm{e}^{x^{2}}$ with increasing $\mathrm{CH}_{4} /$ chamber pressure. Furthermore, all samples show moderate carbon contents and the incipient disordered $\mathrm{Ni}_{3} \mathrm{C}$ phase has a C content of 20(5) \%. This is in good agreement with the previous mentioned results (RPLD film prepared by excimer laser, nitrited samples prepared by Magnetron sputtering) and confirms the modified model.

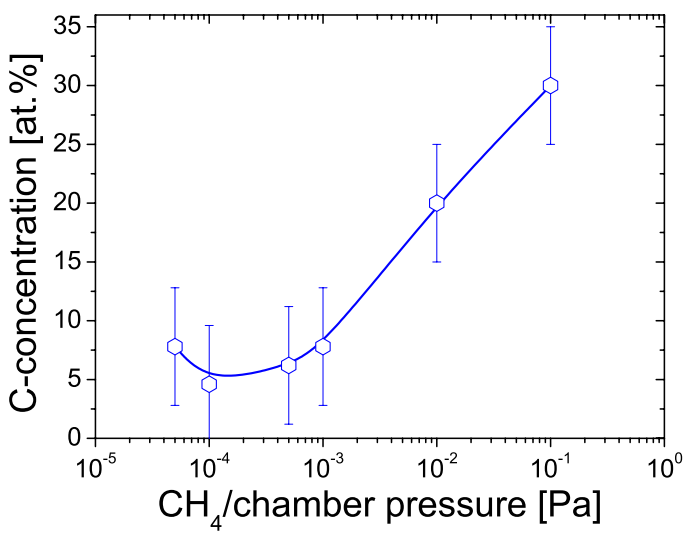

Figure 7.17: Carbon content in the RPLD films (as derived from the RBS analysis) versus the $\mathrm{CH}_{4}$ /chamber pressure.

\subsubsection{Microhardness of the RPLD films}

Nanoindentation was performed by using a Fischerscope HV100 [92] with a Vickers diamond in order to investigate the influence of nitriding on the microhardness and the mechanical properties. The maximum indention force was set to $2 \mathrm{mN}$. Four positions were measured for each sample. The mean values are reported in Table 7.8.

Fig. 7.18 shows the hardness as a function of carbon content. A clear tendency can be seen, where the hardness of the RPLD films increase logarithmical with increasing carbon content and follows the law:

$$
y=2.58(19) \mathrm{GPa}+0.58(9) \cdot \ln (x)
$$

This function approaches asymptotically to the $(\mathrm{Fe}, \mathrm{Cr}, \mathrm{Ni})_{3} \mathrm{C}$ phase.

All experiments of this section confirm the modified nucleation model and indicate that the properties of carburized RPLD films strongly depend on 
Table 7.8: Hardness, Young modulus and Ccontent of RPLD AISI 316 films, where $j$ is the $\mathrm{CH}_{4}$ /chamber pressure, $E$ the elastic modulus an $v$ the poisson ratio.

\begin{tabular}{cccc}
\hline$j$ & Hardness & $\begin{array}{r}\text { Young modulus } \\
\mathrm{E} /\left(1-\mathrm{v}^{2}\right)\end{array}$ & C-content \\
{$[\mathrm{GPa}]$} & {$[$ at. $\%]$} \\
\hline $5 \cdot 10^{-5}$ & {$[\mathrm{GPa}]$} & $68(2)$ & $8(2)$ \\
$1 \cdot 10^{-4}$ & $3.8(4)$ & $86(3)$ & $5(2)$ \\
$1 \cdot 10^{-4}$ & $3.4(5)$ & $54(1)$ & $6(2)$ \\
$1 \cdot 10^{-3}$ & $3.8(1)$ & $70(8)$ & $8(2)$ \\
$1 \cdot 10^{-2}$ & $4.3(3)$ & $71(2)$ & $20(2)$ \\
$1 \cdot 10^{-1}$ & $4.4(7)$ & $68(10)$ & $30(2)$ \\
\hline
\end{tabular}

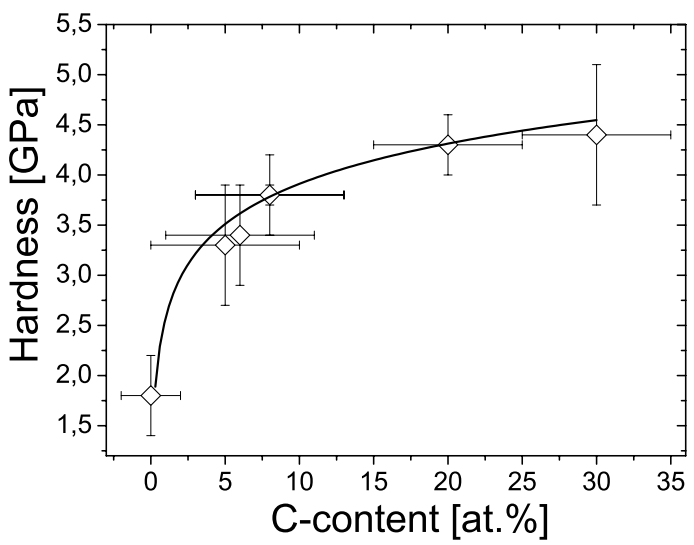

Figure 7.18: Dependency between Martens hardness and carbon content (as derived from the RBS analysis).

the laser density and on the resulting crystallinity of the films.

In the next section, the STPLD technique will be applied on the formation of amorphous stainless steel films.

\subsection{Self-organized structures in carbon-stainless steel multilayer films}

The films were deposited by Sequential Target Pulsed Laser Deposition (STPLD) with a Brilliant Nd:YAG laser $(\lambda=1064 \mathrm{~nm}$, pulse duration 5-6 ns, repetition rate $20 \mathrm{~Hz}$ ) onto amorphous $\mathrm{SiO}_{2}$ substrates (oxidized $\mathrm{Si}(100)$ wafer of $0.5 \mathrm{~mm}$ thickness, pre-cleaned with acetone and oxidized in air, no further treatment) utilizing a commercial AISI 316 (X5CrNiMo17-12-2, 1.4401) target. Before deposition, the PLD-chamber was evacuated to a base pressure of $10^{-6} \mathrm{~Pa}$. The laser energy was set to $5 \mathrm{~mJ} / \mathrm{cm}^{2}$. The targets were pre-combined in that way, that area ratios (of stainless steel to carbon) of
90:10, 80:20, 60:40 and 50:50 were obtained (laser scan area $20 \times 2 \mathrm{~mm}$ on a $25 \times 25 \mathrm{~mm}$ STPLD target - excerpt the 80:20 sample: here the 90:10 STPLD target was used, the desired area ratio was obtained by reducing the laser scan area to $10 \times 2 \mathrm{~mm}$ ). The thickness of the deposited films was controlled by a quartz microbalance (with a film density set to $7.89 \mathrm{~kg} / \mathrm{m}^{3}$, which is the density of AISI 316). The deposition parameters and the resulting thicknesses and growth rates are summarized in Table 7.9.

From these data it is derived that the growth rate

Table 7.9: Deposition time $t$, real film thickness $d$ (as measured by TEM after deposition) and derived growth rate $g=\frac{d}{t}$ for the STPLD films. All samples were deposited at $298 \mathrm{~K}$ with a laser energy of $5 \mathrm{~mJ} / \mathrm{cm}^{2}$ and a target-substrate distance of $7.5 \mathrm{~cm}$.

\begin{tabular}{cccc}
\hline sample & $t$ & $d$ & $g$ \\
& {$[\mathrm{~min}]$} & {$[\mathrm{nm}]$} & {$[\mathrm{nm} / \mathrm{min}]$} \\
\hline Multi90:10 & $26: 52$ & $495(5)$ & $18.42(76)$ \\
Multi80:20 & $20: 37$ & $320(5)$ & $15.52(84)$ \\
Multi60:40 & $25: 35$ & $1074(5)$ & $41.98(173)$ \\
Multi50:50 & $18: 50$ & $371(5)$ & $19.70(118)$ \\
\hline
\end{tabular}

has a Gaussian form with increasing carbon area fraction, which is visualized in Fig. 7.19.

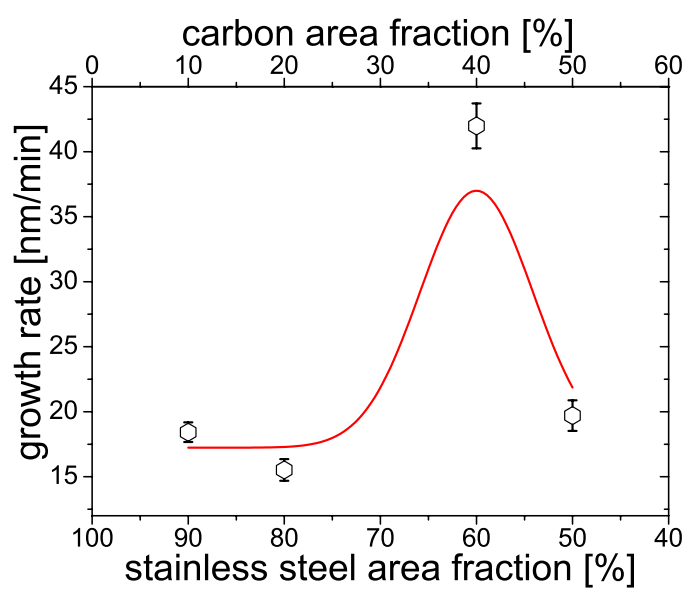

Figure 7.19: Growth rate $g$ as a function of the carbon area fraction for the room temperature STPLD deposition.

\subsubsection{Deposition at room tempera- ture and influence of the car- bon area fraction}

The inlay area, and thus the carbon concentration, was systematically increased from 10 to $50 \%$. For all these deposited films, the STPLD samples show 

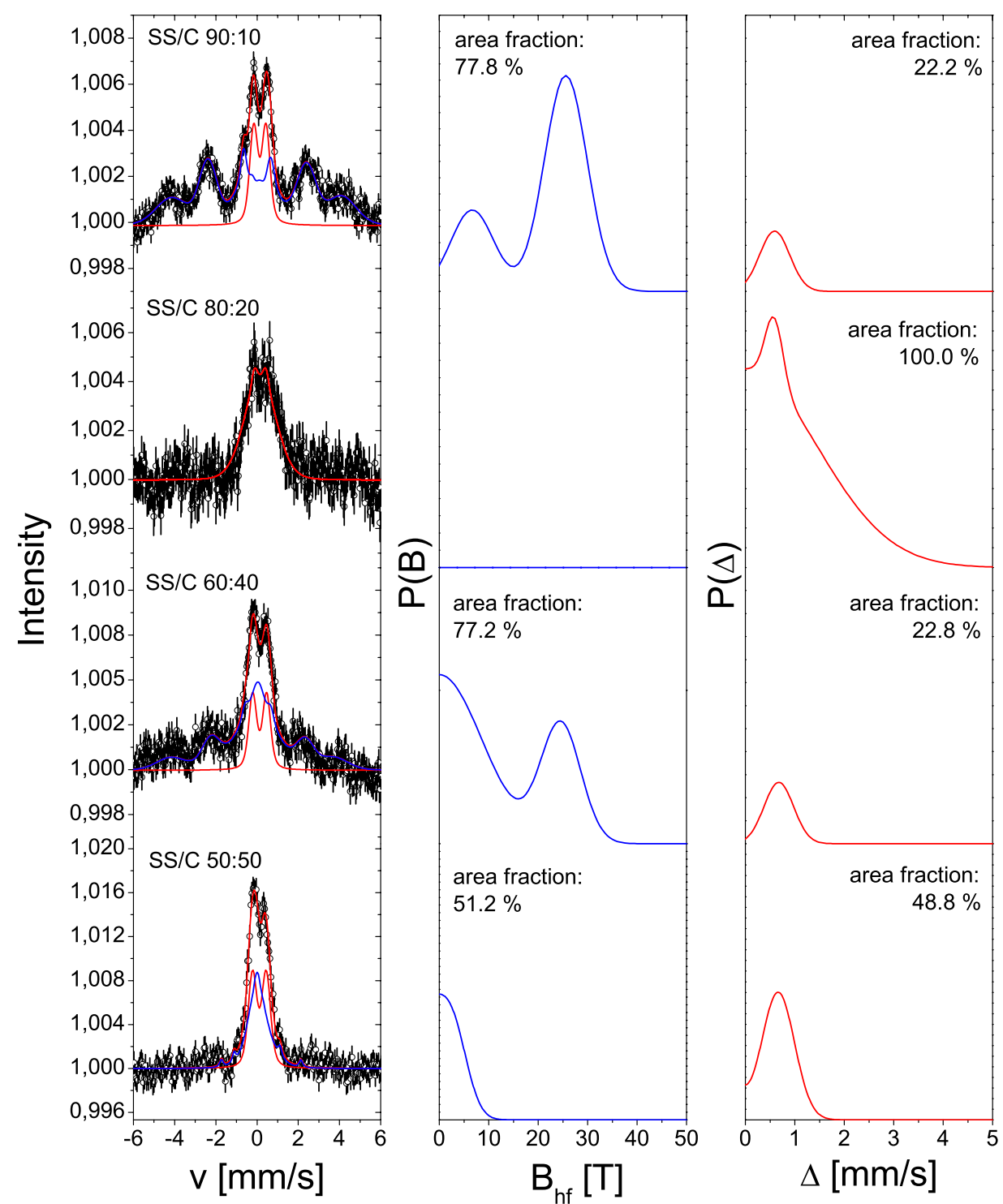

Figure 7.22: Mössbauer spectra of the STPLD stainless steel films. The numbers in the graphs represent the stainless steel/carbon area fraction of the STPLD targets.

the typical broad XRD appearance of amorphous materials, as seen in Fig. 7.20. These diffractograms show a clear amorphous signature.

Fig. 7.21 shows the position and the width of the first broad peak for the spectra shown in Fig. 7.20. There is a clear tendency for peak position and peak width, where the peak position is decreasing (until to SS/C 60:40) and is then increasing rapidly. In contrast, the peak width increases exponentially.

Fig. 7.22 summarizes the CEMS measurements of the samples with increasing gas flow. They show the typical Mössbauer spectra of amorphous materials.

All spectra are - except the 80:20 STPLD sample - magnetically split and are consistent with the XRD results in Fig. 7.20. The results of the single line fitting procedures are presented in Table 7.10. The spectra were fitted by hyperfine field distributions, and with quadrupole splitting distributions in the paramagnetic state, respectively. These distributions are attributed to an amorphous stainless steel carbon-alloy, possibly rich in carbon. The quadrupole splittings are broad and close to the values of the usual carbides and austenite. Not much information can therefore be taken from them. Summarizing, predominantly all samples appear as an amorphous material and show magnetic behavior.

The paramagnetic behavior of the 80:20 STPLD sample is assumed to be originated in the reduced 
Table 7.10: Mössbauer fitting results of the STPLD stainless steel samples, deposited at room temperature with a laser energy of $5 \mathrm{~J} / \mathrm{cm}^{2}(f$ - area fraction (error), mean values of $\langle\delta\rangle$ - isomer shift, $\langle\Delta\rangle-$ the quadrupole splitting for the paramagnetic subspectra, $\langle\epsilon\rangle$ the quadrupole splitting for the magnetic subspectra, $B$ - hyperfine field).

\begin{tabular}{ccrrrrrr}
\hline sample & part & $\begin{array}{r}f \\
{[\%]}\end{array}$ & $\begin{array}{r}\langle\delta\rangle \\
{[\mathrm{mm} / \mathrm{s}]}\end{array}$ & $\begin{array}{r}\langle\Delta\rangle,\langle\epsilon\rangle \\
{[\mathrm{mm} / \mathrm{s}]}\end{array}$ & $\begin{array}{r}\sigma_{\Delta} \\
{[\mathrm{mm} / \mathrm{s}]}\end{array}$ & $\begin{array}{r}\langle B\rangle \\
{[\mathrm{T}]}\end{array}$ & $\begin{array}{r}\sigma_{B} \\
{[\mathrm{~T}]}\end{array}$ \\
\hline \multirow{2}{*}{$90: 10$} & ferro-l & $60.2(9)$ & -0.01 & 0.01 & & $7.7(14)$ & $4.4(14)$ \\
& ferro-h & $17.6(5)$ & -0.01 & 0.01 & & $25.6(4)$ & $4.3(4)$ \\
& para & $22.2(2)$ & 0.14 & 0.59 & 0.29 & & \\
\hline $80: 20$ & para-1 & $92.3(7)$ & 0.15 & 0.01 & 1.00 & & \\
& para-2 & $7.7(3)$ & 0.15 & 0.58 & 0.18 & & \\
\hline $60: 40$ & ferro-l & $46.6(61)$ & -0.09 & -0.11 & & $1.0(10)$ & $10.0(22)$ \\
& ferro-h & $30.6(12)$ & -0.09 & -0.11 & & $24.5(18)$ & $4.1(12)$ \\
& para & $22.8(5)$ & 0.13 & 0.67 & 0.29 & & \\
\hline $50: 50$ & ferro-l & $5.1(5)$ & 0.10 & 0.10 & & $2.6(12)$ & $3.0(22)$ \\
& ferro-h & $46.1(7)$ & 0.10 & 0.10 & & $11.9(7)$ & $0.1(36)$ \\
& para & $48.8(1)$ & 0.11 & 0.67 & 0.32 & & \\
\hline
\end{tabular}
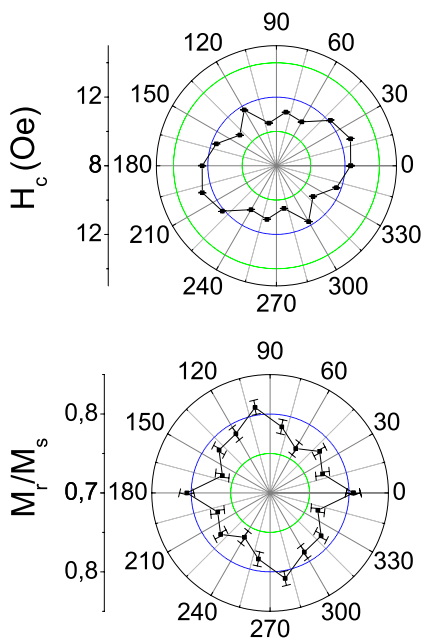
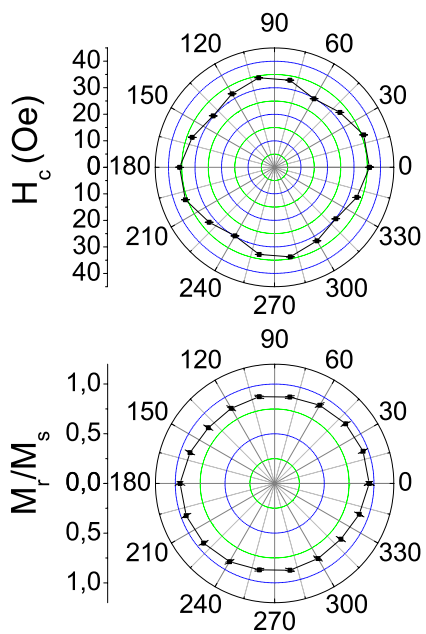
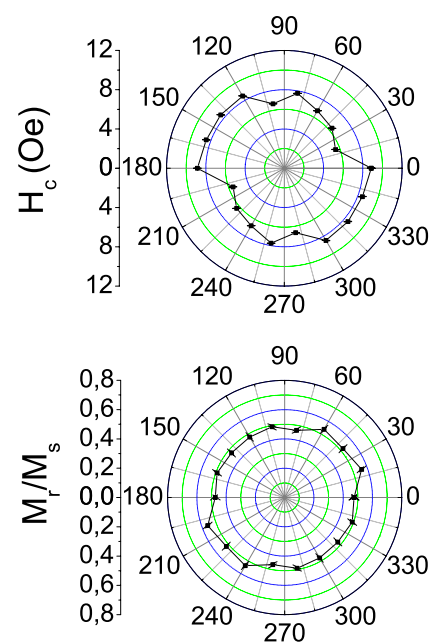

Figure 7.23: Polar diagrams of the coercive field $H_{\mathrm{C}}$ and the relative remanence $M_{\mathrm{R}} / M_{\mathrm{S}}$ for the STPLD 90:10 (left), the 60:40 (middle) and the 50:50 sample (right).

scan area and, thus, an increase of laser scan speed, which could be an important parameter (this will be investigated in the next section).

Mössbauer results were confirmed by MOKE results, which are presented in Fig. 7.23.

While the samples with 90:10, 60:40 and 50:50 exhibit a MOKE signal, the sample 80:20 does not appear magnetic in MOKE. The 90:10 sample exhibits a uniaxial anisotropy at $20^{\circ}$ and additionally a sixfold anisotropy. According to Ref. [88,89], this can be attributed to epitaxial growth. In contrast to the 90:10 sample, the 60:40 and 50:50 specimens exhibit fourfold anisotropy. The coercive fields $\left(H_{C}\right)$ differ: the specimen 90:10 and 50:50 show soft ferromagnetic behaviour $\left(H_{C} \approx 10 \mathrm{Oe}\right)$, in contrast to the 60:40 specimen, which show harder ferromagnetism $\left(H_{C} \approx 35 \mathrm{Oe}\right)$. These observations have now to be correlated to the carbon content of the film.

The EDX analyses of the deposited films revealed the original composition of the sputtering targets within the experimental limits. Unfortunately, the EDX did not allow to accurately determine elements lighter than oxygen. Therefore, in order to evaluate the amount of incorporated carbon, RBS measurements were carried out, whose results are shown in Fig. 7.24.

The thickness of the films as obtained from the RBS analysis are in good agreement of those derived by TEM, which were given in Table 7.9. However, the RBS spectra show an exceptionally oscillating behavior.

The oscillating characteristics in the spectra indicate that the films consist of a multilayer system. A first RBS fitting tool developed for high-resolution RBS spectra was used to get a concentration depth profile [142]. As an example, the fitting result for the 90:10 specimen is illustrated in Fig.7.25.

A periodic sequence of alternating stainless-steel and $C$ layers can be observed. The thickness of the steel layer is about $25 \mathrm{~nm}$ and that of the carbon 

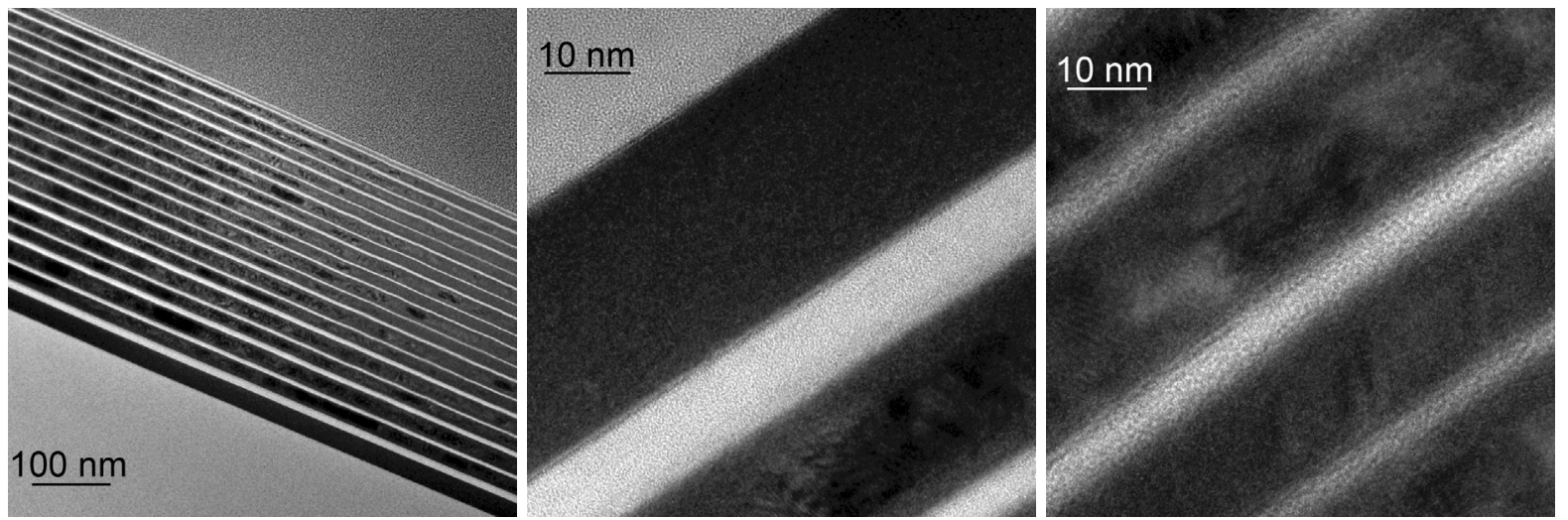

Figure 7.26: TEM pattern of the STPLD 9010 sample. Left: overview of the sample; middle: HR-TEM image of film/interface region; right: HR-TEM image of the surface region.

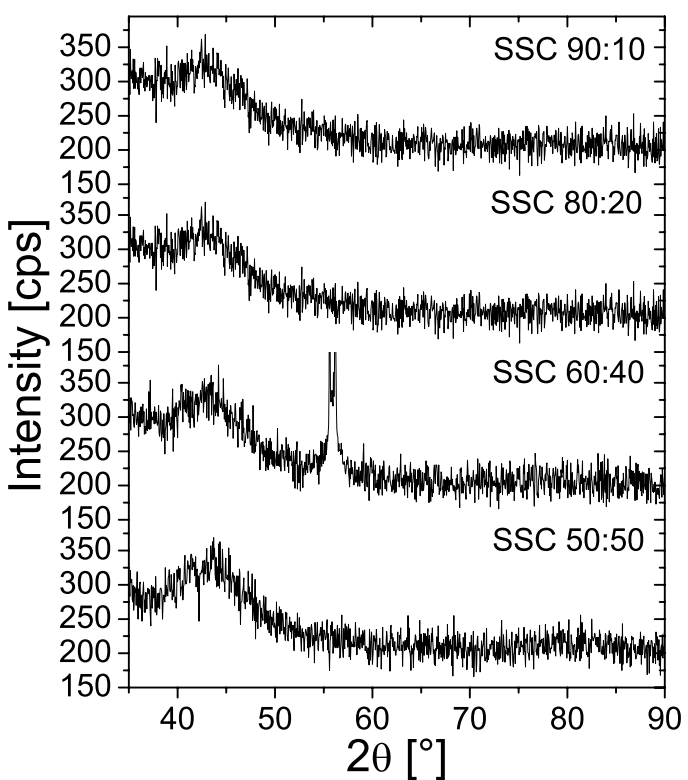

Figure 7.20: GIXRD $\left(2^{\circ}\right)$ spectra of the STPLD films. The stainless steel/carbon area fractions are given in the graph.

layer $15 \mathrm{~nm}$. It can be also seen that the layer thickness of the 80:20 sample is smaller than in the other samples and that the laser scanning frequency could be a crucial factor for the multilayer formation, as already mentioned by Mössbauer Spectroscopy. Thus, a dependency between film properties such as magnetism/microstructure and layer growth is assumed. Another problem is presented by the number of multilayer: by knowing the complete deposition time and the time for one laser scan cycle, the numer of the multilayer can be estimated. In all samples this number does not correspond to the observed numbers of multilayer. This phenomenon will be discussed later, wherein an induced selforganizing effect will be revealed.

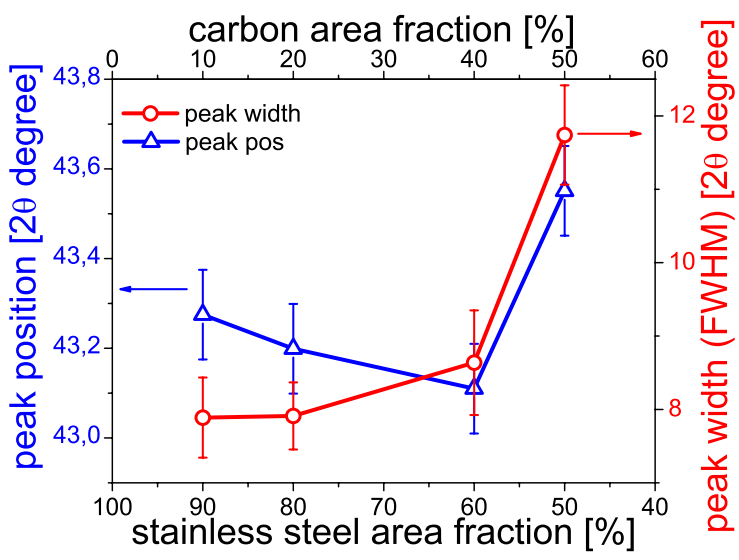

Figure 7.21: Peak position and peak width of the first broad peak in the XRD spectra of the STPLD stainless steel films.

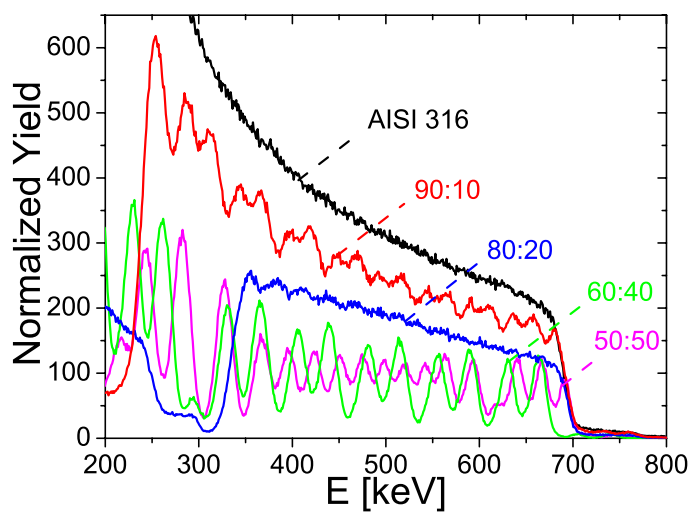

Figure 7.24: RBS spectra of the STPLD films. The stainless steel/carbon area ratios are given in the graph.

To achieve a higher resolution, high-resolution Rutherford backscattering spectrometry (HR-RBS) 


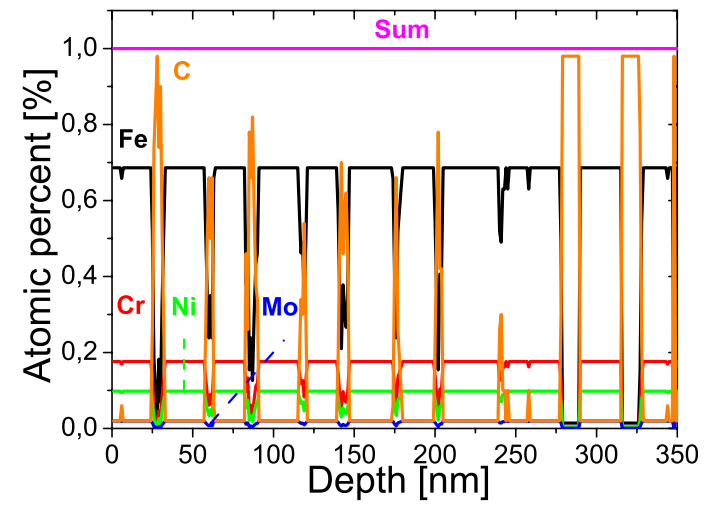

Figure 7.25: RBS depth profile of the STPLD 90:10 sample.

measurements were carried out, but no difference between conventional RBS and HR-RBS was found. Thus, TEM patterns were taken, which are depicted in Fig. 7.26.

The TEM patterns of all STPLD samples confirm the RBS results, but one characteristic can be taken from them: all samples show a gradually decrease of the multilayer thickness during deposition and it seems to be a constant decreasing factor of about $1 / 3$. Also, the quality of the multilayer near the surface is decreasing. The observed characteristics are originated in the cavation of the target by the laser beam, which induces a shift of the laser plasma plume. For this, experiments using a target-wobbler system were performed, but as next step a dependency between scan frequency and multilayer thickness is investigated.

\subsubsection{Influence of the scan frequency on the multilayer thickness}

To investigate the dependency between scan frequency and multilayer thickness of high-carbon stainless steel films, STPLD 60:40 and 50:50 targets were combined in the way as displayed in Fig. 7.1 and labeled as 60:40mod and 50:50mod, respectively.

Drastic changes can be seen in the CEM spectra: whereas the 60:40 spectrum show both - magnetic and paramagnetic - sites, the 60:40mod spectrum exhibit only a broad paramagnetic site indicating a high carbon concentration. The quadrupole splittings are very close to the values of the usual carbides, but no further information can therefore be taken from them. The absence of magnetism is also observed in MOKE measurements which exhibit no MOKE signal. The results of the fitting procedures are presented in Table 7.11.
Also the TEM patterns show significant changes in the multilayer structure: whereas the 60:40 STPLD sample show $50 \mathrm{~nm}$ thick stainless steel layers and $30 \mathrm{~nm}$ C layers, the 60:40mod STPLD sample consists of very thin layers $(\sim 4-8 \mathrm{~nm})$, which form a superstructure of about $25 \mathrm{~nm}$. The TEM pattern for the 60:40mod STPLD sample exhibit a high amount of diffusion. As a consequence, no clear and well defined multilayer structure can be seen. Furthermore, no crystallites were seen in the 60:40mod STPLD sample as seen in TEM patterns of the 60:40 STPLD sample.

TEM results were partiallyconfirmed by RBS. Only the superstructure can be observed, which has a layer thicknes of about $25 \mathrm{~nm}$. Noticeable is the carbon content of 60:40mod STPLD sample, which was derived by an advanced and enhanced fitting tool [143]: about $50 \%$ carbon content are observed $10 \%$ more than in conventional 60:40 STPLD films. Also here the number of multilayers do not correspond to scan process of the laser beam, but the discrepancy is not as high as observed in the 90:10 STPLD sample.

As a first conclusion it can be stressed out, that the modification of STPLD targets lead to the formation of nano-scaled multilayers.

Fig. 7.28 shows a comparison between the results of conventional and modified 50:50 STPLD films, which exhibit similar properties. The CEM spectrum of the modified 50:50 STPLD film do not reveal magnetic properties (as also observed for the 60:40mod STPLD film). The paramagnetic site is broad; even broader than the site in the $60: 40 \mathrm{mod}$ STPLD film and also indicating a higher carbon content. The CEMS results were confirmed by MOKE, which exhibit no MOKE signal. The results of the fitting procedures are presented in Table 7.11 .

As seen in the TEM patterns of the $60: 40 \mathrm{mod}$ STPLD film, the 50:50mod STPLD film exhibit a similar multilayer structure, whereas the 50:50 STPLD sample show an alternating multilayer system consisting of $30-50 \mathrm{~nm}$ stainless steel and 20-30 $\mathrm{nm}$ carbon layers. The layers in the 50:50mod sample can be better distinguished than the 60:40mod STPLD film, but carbon diffusion can also be observed. The thin layer thickness can be derived to $\sim 3-6 \mathrm{~nm}$.

RBS results for the 50:50 and 50:50mod STPLS films are depicted in the bottom of Fig. 7.28 and exhibit the same superstructure as seen for the 60:40mod film. The superstructure exhibit a layer thickness of about $20 \mathrm{~nm}$. The median carbon content of $50 \%$ as shown in the depth profile, was also observed in the 60:40mod film. The difference between the $60: 40 \mathrm{mod}$ and $50: 50 \mathrm{mod}$ films is the solved carbon in the steel matrix. Whereas in the 
Table 7.11: Mössbauer fitting results of the 60:40, 60:40mod, 50:50 and 50:50mod STPLD samples, deposited at room temperature with a laser energy of $5 \mathrm{~J} / \mathrm{cm}^{2}$ ( $f$ - area fraction (error), mean values of $\langle\delta\rangle$ - isomer shift, $\langle\Delta\rangle$ - the quadrupole splitting for the paramagnetic subspectra, $\langle\epsilon\rangle$ the quadrupole splitting for the magnetic subspectra, $B$ - hyperfine field).

\begin{tabular}{ccrrrrrr}
\hline sample & part & $\begin{array}{r}f \\
{[\%]}\end{array}$ & $\begin{array}{r}\langle\delta\rangle \\
{[\mathrm{mm} / \mathrm{s}]}\end{array}$ & $\begin{array}{c}\langle\Delta\rangle,\langle\epsilon\rangle \\
{[\mathrm{mm} / \mathrm{s}]}\end{array}$ & $\begin{array}{r}\sigma_{\Delta} \\
{[\mathrm{mm} / \mathrm{s}]}\end{array}$ & $\begin{array}{r}\langle B\rangle \\
{[\mathrm{T}]}\end{array}$ & $\begin{array}{r}\sigma_{B} \\
{[\mathrm{~T}]}\end{array}$ \\
\hline \multirow{2}{*}{$60: 40$} & ferro-l & $46.6(61)$ & -0.09 & -0.11 & & $1.0(10)$ & $10.0(22)$ \\
& ferro-h & $30.6(12)$ & -0.09 & -0.11 & & $24.5(18)$ & $4.1(12)$ \\
& para & $22.8(5)$ & 0.13 & 0.67 & 0.29 & & \\
\hline $60: 40 \mathrm{mod}$ & para & $100.0(1)$ & 0.23 & 0.76 & 0.44 & & \\
\hline $50: 50$ & ferro-l & $5.1(5)$ & 0.10 & 0.10 & & $2.6(12)$ & $3.0(22)$ \\
& ferro-h & $46.1(7)$ & 0.10 & 0.10 & & $11.9(7)$ & $0.1(36)$ \\
& para & $48.8(1)$ & 0.11 & 0.67 & 0.32 & & \\
\hline $50: 50 \bmod$ & para-1 & $7.6(13)$ & 0.22 & 0.58 & 0.20 & & \\
& para-2 & $92.5(74)$ & 0.22 & 0.85 & 0.49 & & \\
\hline
\end{tabular}

60:40mod film are only solved $20-25 \%$ carbon in the metal matrix, 30-35\% are solved in 50:50mod films, which results in a lower RBS-yield for the 50:50mod spectrum.

Fig. 7.27 shows a comparison between the results of conventional and modified 60:40 STPLD films.

Finally it could be shown, that the laser scan frequency has a crucial influence on the formation of carbon-stainless steel multilayer and that it is an important parameter to control the multilayer thickness. 

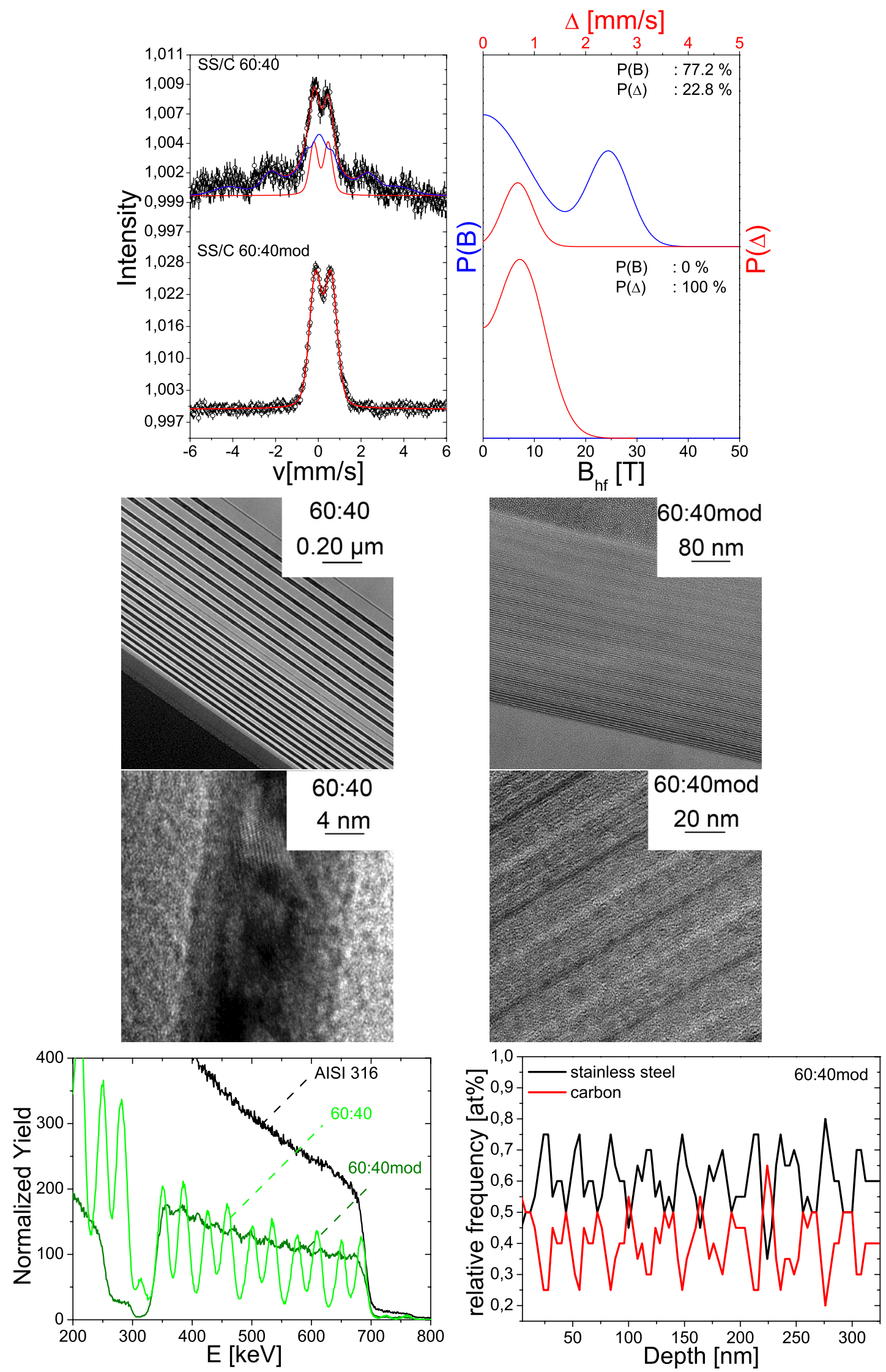

Figure 7.27: Comparison between conventional and modified 60:40 STPLD films: Mössbauer spectra (top); TEM pattern (middle) and RBS spectra with calculated depth profile (bottom). 

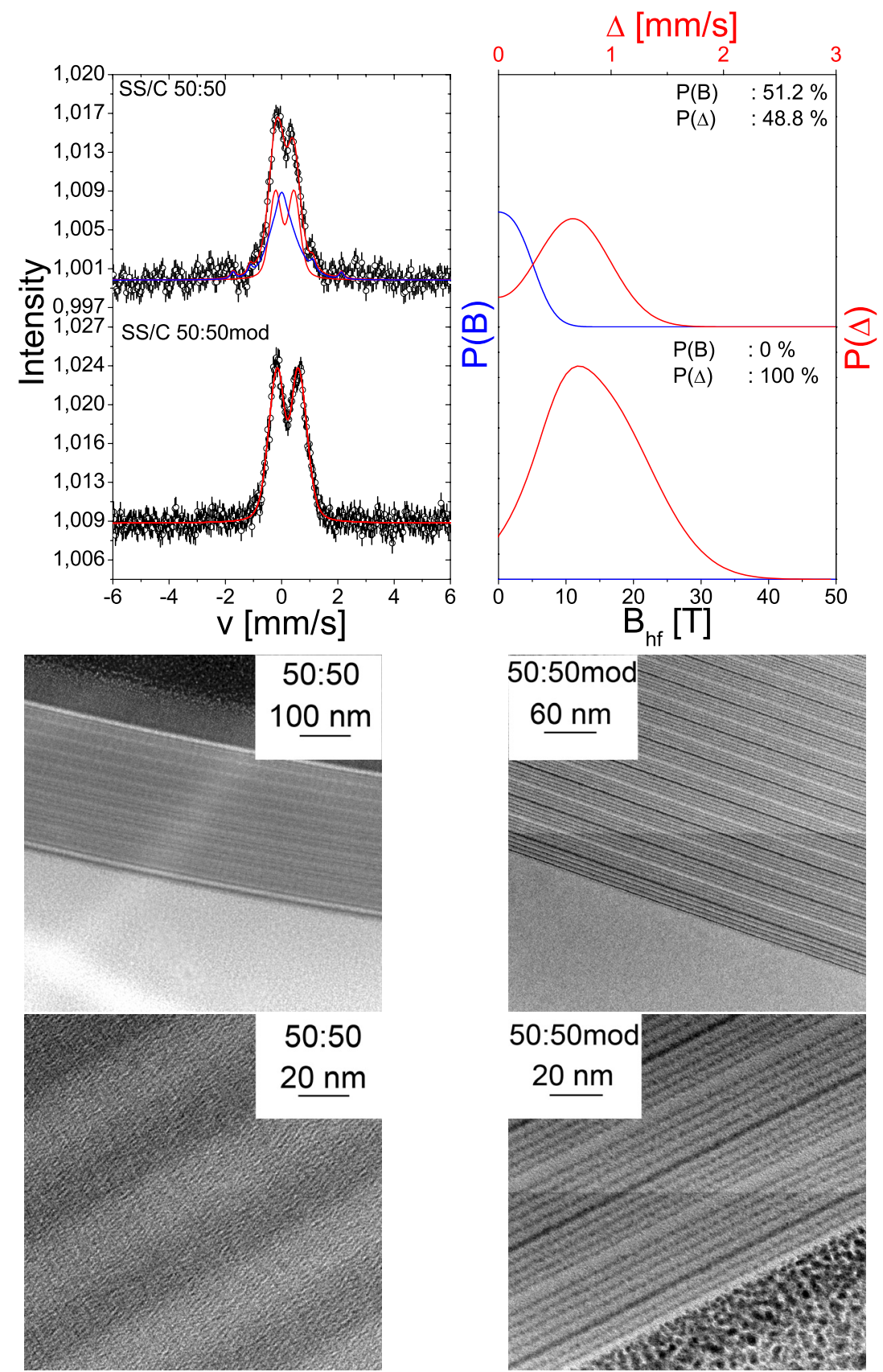

\section{0:50mod}
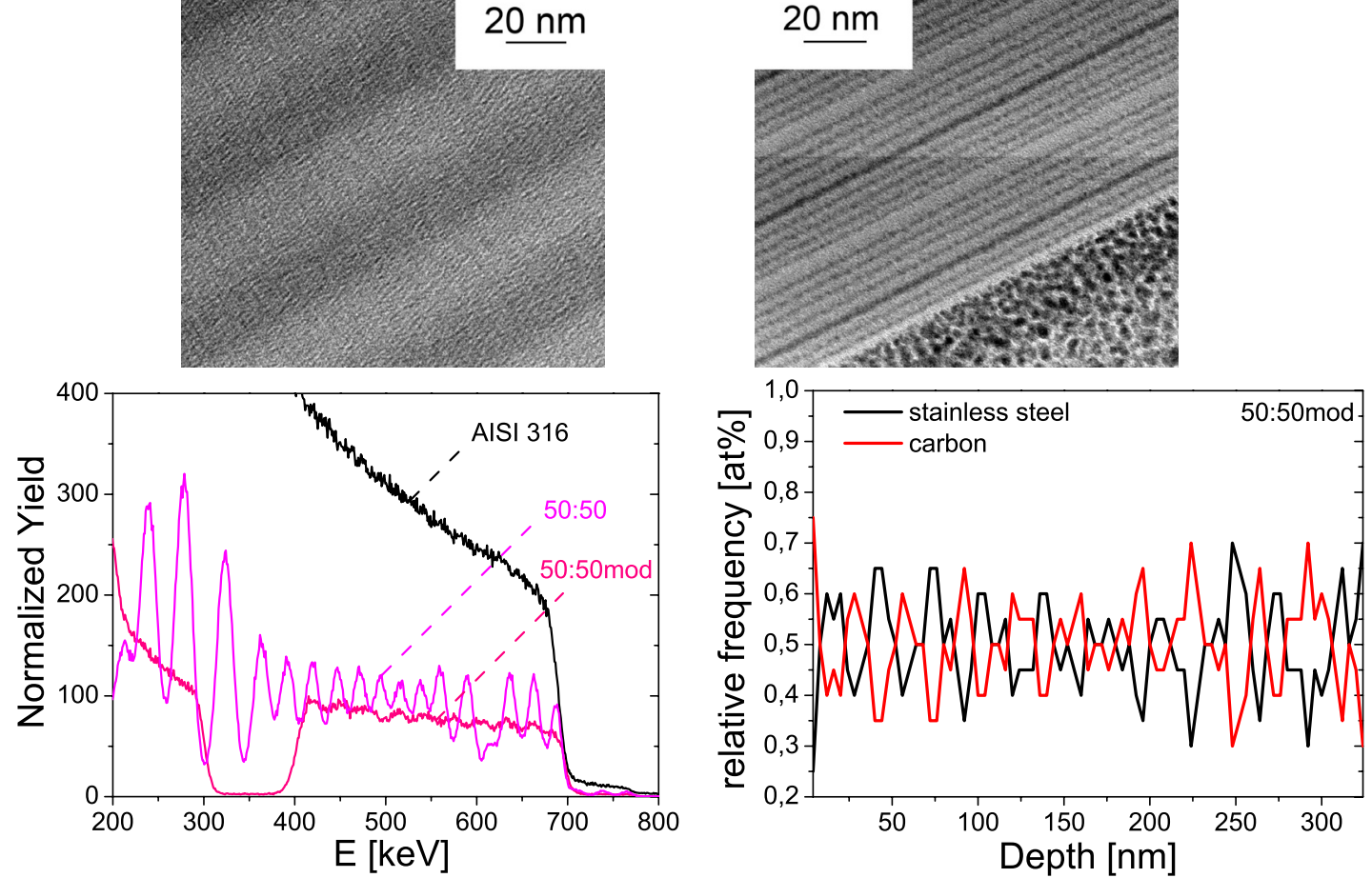

Figure 7.28: Comparison between conventional and modified 50:50 STPLD films: Mössbauer spectra (top); TEM pattern (middle) and RBS spectra with calculated depth profile (bottom). 


\subsubsection{Thermal stability of carbon- stainless steel multilayer films}

Vacuum annealing of carbon-stainless steel multilayer films In order to analyze the thermal stability of carbon - stainless steel multilayers, vacuum annealing at a temperature of $973 \mathrm{~K}$ for 1 hour was performed for the 90:10 STPLD film.

Fig. 7.29 shows the XRD pattern of the vacuum annealed 90:10 film.

The GIXRD pattern exhibit two crystallized

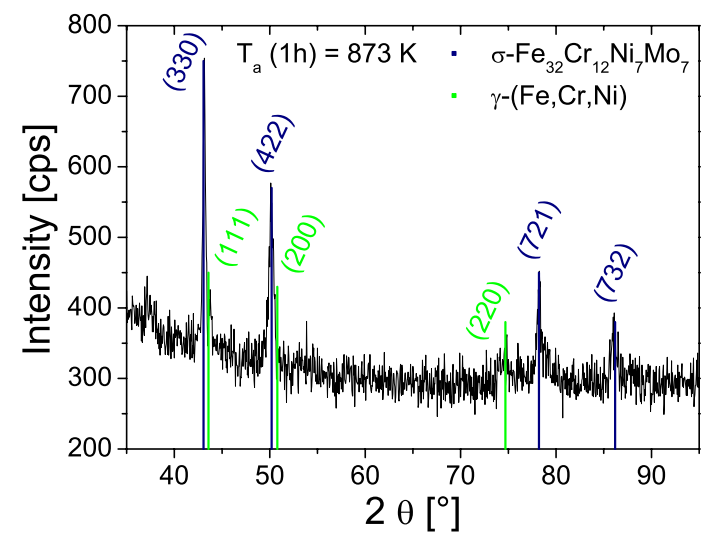

Figure 7.29: GIXRD ( $2^{\circ}$ incidence angle) spectrum of the post-vacuum annealed STPLD film. Phase, annealing temperature and time are given in the graph.

phases: the $\gamma-(\mathrm{Fe}, \mathrm{Cr}, \mathrm{Ni})$ phase $(a=0.359(2) \mathrm{nm})$ and the $\mathrm{BCC} \sigma-(\mathrm{Fe}, \mathrm{Cr}, \mathrm{Ni})(a=0.892(3) \mathrm{nm})$, which corresponds to the $\mathrm{Fe}_{32} \mathrm{Cr}_{10} \mathrm{Ni}_{7} \mathrm{Mo}_{7}$ steel. As a consequence, magnetic subspectra are expected in the CEM spectrum, which is depicted in Fig. 7.30 [144].

The CEM spectrum reveals, that the post-vacuum
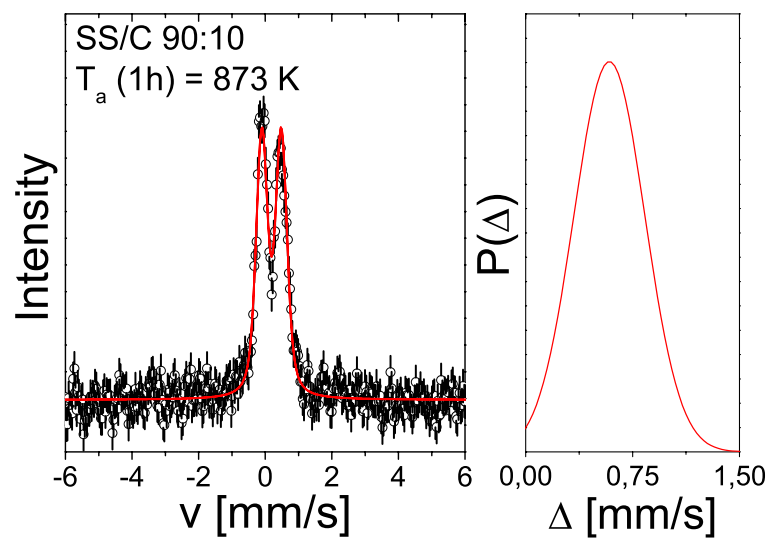

Figure 7.30: CEM spectrum of the post-vacuum annealed 90:10 STPLD film.

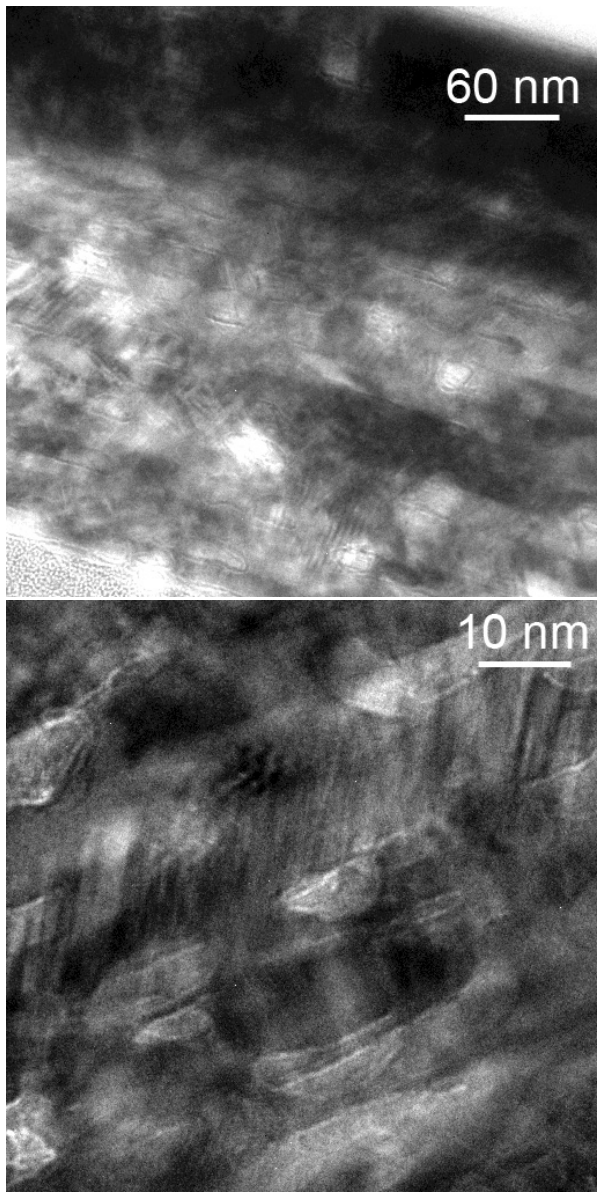

Figure 7.31: TEM patterns of the post-vacuum annealed 90:10 STPLD film.

annealed 90:10 film is in the paramagnetic state in contrast to the as-deposited 90:10 sample, which exhibit both magnetic and paramagnetic subspectra. The hyperfine parameters after annealing for $1 \mathrm{~h}$ are well-defined, correspond to austenite and are presented in Table 7.12.

The differences in CEMS and XRD can be explained by the unequal information depths of both methods (CEMS: $\sim 150 \mathrm{~nm} ; 2^{\circ}$ GIXRD: $\sim 350 \mathrm{~nm}$ ). As a result, the magnetic $\sigma-(\mathrm{Fe}, \mathrm{Cr}, \mathrm{Ni})$ phase can only be observed in deeper regions, as detected by GIXRD.

This is confirmed by TEM which is illustrated in Fig. 7.31 and which show, in contrast to the asdeposited 90:10 film, that the multilayer structure pry open (and thus no clear multilayer system is observed) due to carbon diffusion during annealing. Furthermore, a segregation of the metal atoms to the film/substrate interface can be seen. An EDX analysis showed that the composition of the film has changed dramatically. In addition, the composition is not homogenous in the film; every dark area in the TEM pattern has another composition, but 
Table 7.12: Mössbauer fitting results of the STPLD stainless steel samples, deposited at room temperature $\left(90: 10_{a d}\right)$ and after post-vacuum annealing $\left(90: 10_{p v a}\right): f$ - area fraction (error), mean values of $\langle\delta\rangle$ - isomer shift, $\langle\Delta\rangle$ - the quadrupole splitting for the paramagnetic subspectra, $\langle\epsilon\rangle$ the quadrupole splitting for the magnetic subspectra, $B$ - hyperfine field.

\begin{tabular}{ccrrrrrr}
\hline sample & part & $f$ & $\langle\delta\rangle$ & $\langle\Delta\rangle,\langle\epsilon\rangle$ & $\sigma_{\Delta}$ & $\langle B\rangle$ & $\sigma_{B}$ \\
& & {$[\%]$} & {$[\mathrm{mm} / \mathrm{s}]$} & {$[\mathrm{mm} / \mathrm{s}]$} & {$[\mathrm{mm} / \mathrm{s}]$} & {$[\mathrm{T}]$} & {$[\mathrm{T}]$} \\
\hline $90: 10_{a d}$ & ferro-l & $60.2(9)$ & -0.01 & 0.01 & & $7.7(14)$ & $4.4(14)$ \\
& ferro-h & $17.6(5)$ & -0.01 & 0.01 & & $25.6(4)$ & $4.3(4)$ \\
& para & $22.2(2)$ & 0.14 & 0.59 & 0.29 & & \\
\hline $90: 10_{\text {pva }}$ & para & $100.0(1)$ & 0.19 & 0.58 & 0.25 & & \\
\hline
\end{tabular}

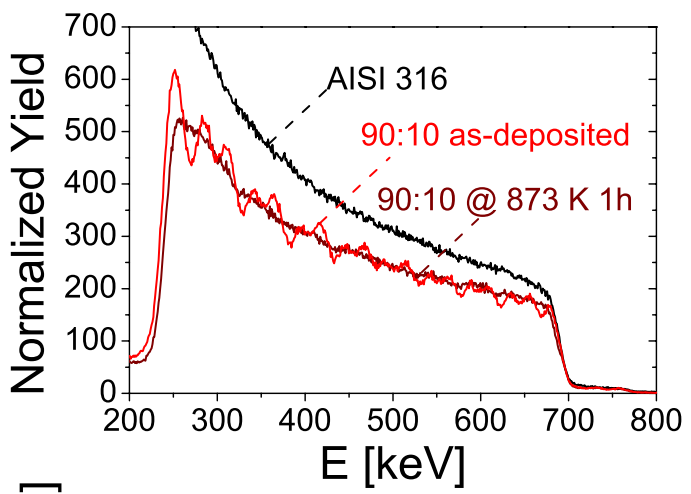

propriate technique to improve the properties of carbon - stainless steel multilayers. As a consequence, the influence of substrate temperature during deposition has to be investigated. The results of those experiments are presented in the next section.

Influence of the substrate temperature on the properties of carbon - stainless steel multilayers To investigate the influence of substrate temperature on the properties of carbon - stainless steel multilayer, a 90:10 film with a substrate temperature of $673 \mathrm{~K}$ was deposited.

The GIXRD pattern, as shown in Fig. 7.33, exhibit

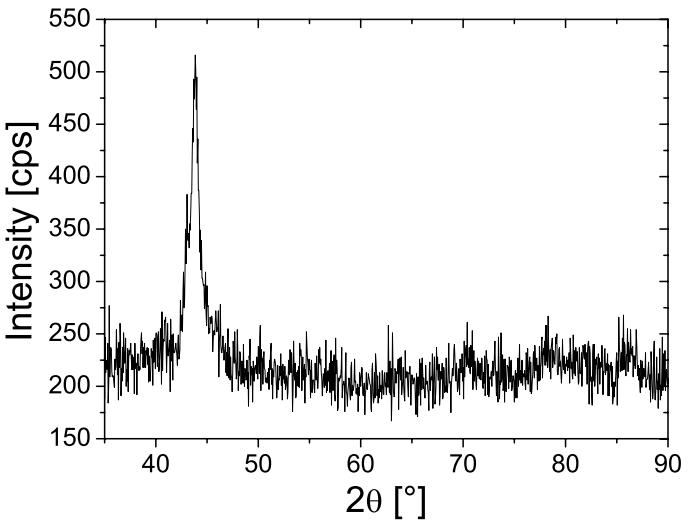

Figure 7.32: RBS spectra of the post-vacuum annealed and as-deposited 90:10 STPLD film. the depth profile of the post-vacuum annealed can be found at the bottom

as indicated by GIXRD, the Mo concentration is higher than in the initial target. This could be due to different diffusion constants of the metal atoms. RBS measurements were carried out to derive the carbon content. The results - spectra and depth profile - can be seen in Fig. 7.32.

RBS confirms the TEM results. A broad multilayer structure can be seen in the spectrum for the post-vacuum annealed film with varying layer thicknesses, which can be also observed in the calculated depth profile. The carbon content approximates the content as seen in the as-deposited 90:10 film.

Summarizing, post-vacuum annealing is not an ap-

Figure 7.33: GIXRD ( $2^{\circ}$ incidence angle) spectrum of the 90:10 STPLD film deposited with an substrate temperature of $673 \mathrm{~K}$.

the typical shape of amorphous materials. Whereas the peak position has not changed (in comparison to the 90:10 film deposited at room temperature), the peak width has decreased indicating appendages of crystallization. This can also be seen for higher $2 \theta$-values, where small peaks protrude from the amorphous underground (and could possibly be attributed to an $\alpha$ - and a $\gamma$-phase. As a consequence, the film could be nano-crystalline.

The CEM spectrum is depicted in Fig. 7.34.

Some significant changes can be observed for the 90:10 film deposited with a substrate temperature 

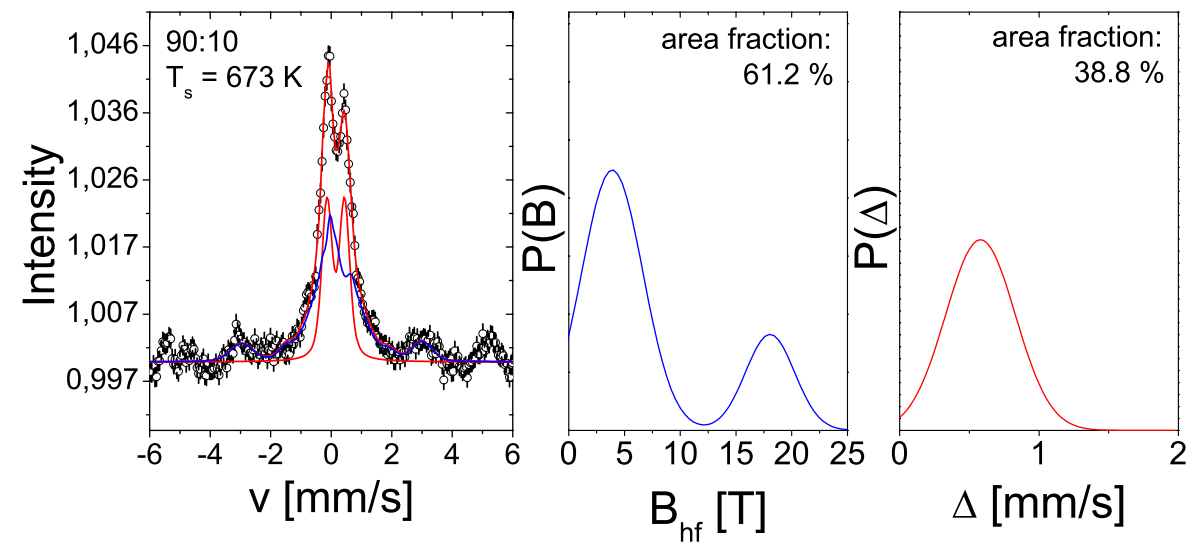

Figure 7.34: CEM spectrum of the 90:10 STPLD film deposited with an substrate temperature of 673 K.

Table 7.13: Mössbauer fitting results of the 90:10 STPLD stainless steel samples, deposited at room temperature and at $673 \mathrm{~K}$ ( $f$ - area fraction (error), mean values of $\langle\delta\rangle$ - isomer shift, $\langle\Delta\rangle$ - the quadrupole splitting for the paramagnetic subspectra, $\langle\epsilon\rangle$ the quadrupole splitting for the magnetic subspectra, $B$ hyperfine field).

\begin{tabular}{ccrrrrrr}
\hline $\mathrm{T}$ & part & $\begin{array}{r}f \\
{[\%]}\end{array}$ & $\begin{array}{r}\langle\delta\rangle \\
{[\mathrm{mm} / \mathrm{s}]}\end{array}$ & $\begin{array}{l}\langle\Delta\rangle,\langle\epsilon\rangle \\
{[\mathrm{mm} / \mathrm{s}]}\end{array}$ & $\begin{array}{r}\sigma_{\Delta} \\
{[\mathrm{mm} / \mathrm{s}]}\end{array}$ & $\begin{array}{r}\langle B\rangle \\
{[\mathrm{T}]}\end{array}$ & $\begin{array}{r}\sigma_{B} \\
{[\mathrm{~T}]}\end{array}$ \\
\hline 298 & ferro-l & $60.2(9)$ & -0.01 & 0.01 & & $7.7(14)$ & $4.4(14)$ \\
& ferro-h & $17.6(5)$ & -0.01 & 0.01 & & $25.6(4)$ & $4.3(4)$ \\
& para & $22.2(2)$ & 0.14 & 0.59 & 0.29 & & \\
\hline 673 & ferro-l & $47.0(50)$ & 0.11 & 0.03 & & $3.9(3)$ & $2.9(4)$ \\
& ferro-h & $14.2(10)$ & 0.11 & 0.03 & & $18.5(7)$ & $2.0(4)$ \\
& para & $38.8(2)$ & 0.15 & 0.58 & 0.20 & & \\
\hline
\end{tabular}

of $673 \mathrm{~K}$. Although, the film exhibit a magnetic split sextet, but its area fraction is decreased in comparison to the 90:10 film deposited at room temperature. Furthermore, changes in the hyperfine distribution can be seen: whereas in the room temperature film the probability for bigger fields is higher, the film deposited at $673 \mathrm{~K}$ exhibits a hiher probability for small fields, which could be attributed to an amorphous stainless steel carbon-alloy, possibly rich in carbon. The amount of the quadrupole splitting is here increased indicating a transformation to austenite or carbides. due to the broadness of the quadrupole splitting, no more information can be taken from them. The results of the fitting procedures are presented in Table 7.13.

Although the CEM spectrum exhibit magnetically split sextets, no MOKE signal is detected. Since MOKE is sensitive to the first $5-10 \mathrm{~nm}$, it is reasonable to assume, that the magnetic grains are found in deeper regions.

Again RBS measurements were carried out to investigate the carbon content and to get a first estimation about the multilayer structure. The results are presented in Fig. 7.35.

The carbon content in the sample deposited at $673 \mathrm{~K}$ is verisimilar to that film deposited at room
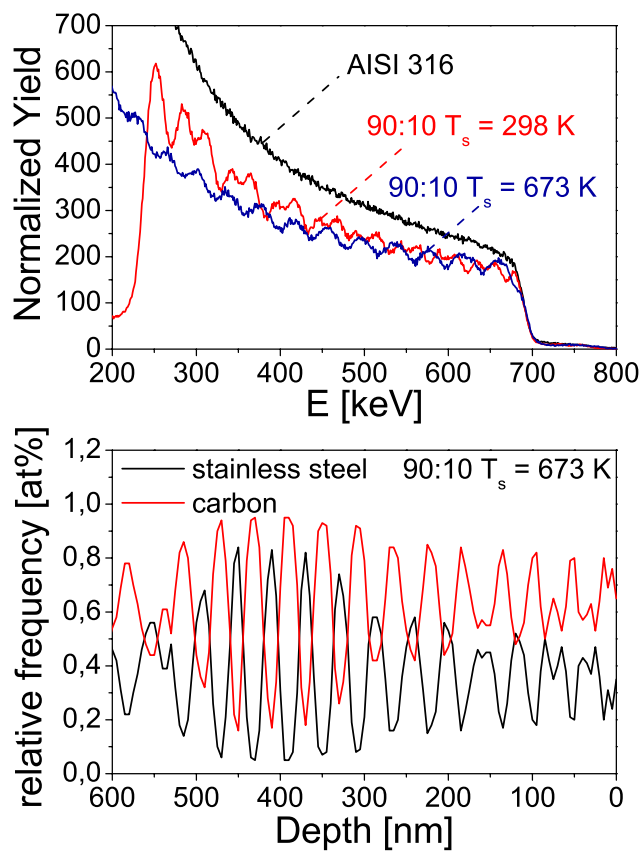

Figure 7.35: RBS spectra of the 90:10 STPLD film deposited at 298 and $673 \mathrm{~K}$. The depth profile of the film deposited at $673 \mathrm{~K}$ can be found at the bottom. 

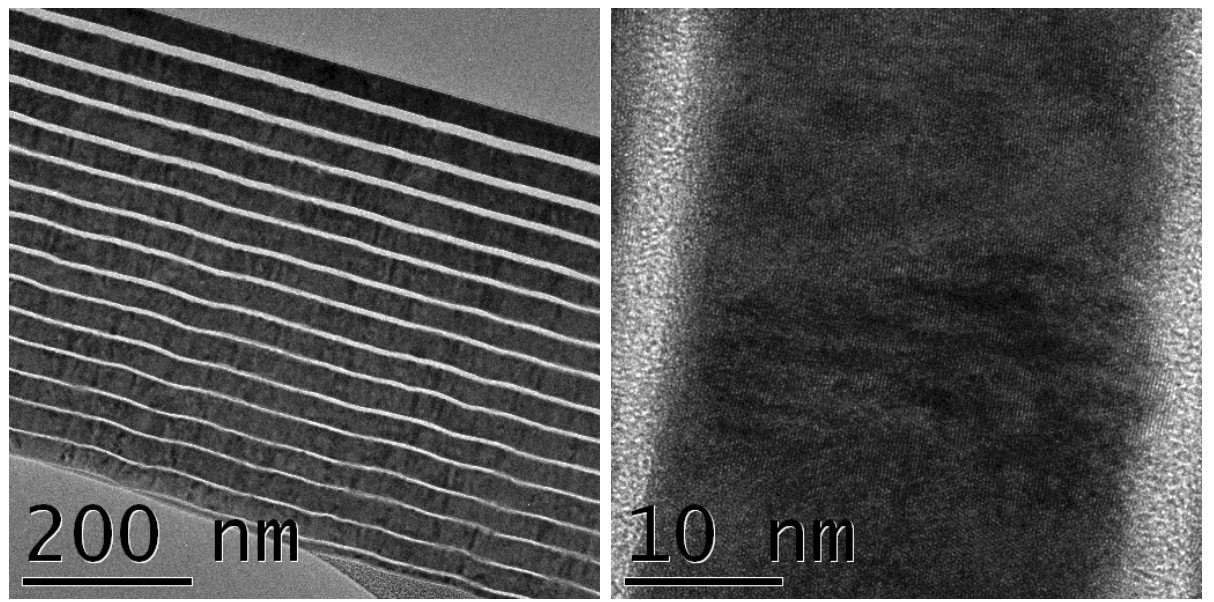

Figure 7.36: TEM patterns of the 90:10 STPLD sample deposited at $673 \mathrm{~K}$. Left: overview of the sample; right: HR-TEM pattern.

temperature. The depth profile show well defined multilayer, which are verisimilar in thickness. Also the decrease of the multilayer thickness seems to be less severe than in room temperature depositions. The beat in the oscillation at a depth of 300-500 nm is not a result of diffusion processes in deposition, but can be rather attributed to straggling effects during the RBS measurement.

RBS results were confirmed by TEM patterns, which are shown in Fig. 7.36.

Except for small waves between the layer interfaces of carbon and stainless steel, the multilayer structure is well defined; even better than in room temperature deposition. Small crystallites can be observed in the HR-TEM image, but no SAD patterns could be taken indicating the poor thermal stability of the grains, which is in good agreement to the results for post-vacuum annealed samples (an electron beam, focussed between 5-30 keV, induces structure modifications in a mostly amorphous metal matrix up to $20 \mathrm{~nm}$; as a consequence the grains can disappear). As indicated by RBS, the decrease of the layer thickness to the surface is less severe than for room temperature depositions.

Summarizing, the deposition at higher substrate temperatures leads to an improvement of the layer thickness and homogeneity. Future experiments should find the ideal substrate temperature to deposit clear and well defined carbon - stainless steel multilayer.

\subsubsection{The quality of the carbon- stainless steel multilayer}

As mentioned in the previous section, substrate temperature has a non insignificant influence on the multilayer structure. Thus, it is necessary to char- acterize the morphology of the multilayer systems and to find methods to improve their properties.

From an industrial point of view, multilayer systems at the nano-scale would be interesting. As derived from the TEM analysis, the 50:50mod STPLD film seems to answer this purpose. As a consequence, Xray reflectivity (XRR) experiments were performed and analyzed with the IMD extension pack of the XOP X-ray optics software toolkit $[145,146]$. The result is depicted in Fig. 7.37.

The analysis exhibits a superstructure, which is

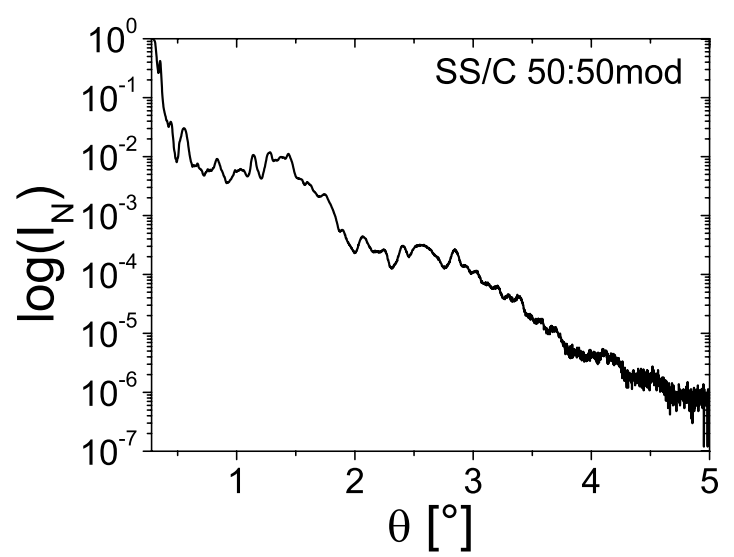

Figure 7.37: X-ray reflectivity pattern of the 50:50mod STPLD film.

corresponding to the RBS results. The superstructure has a thickness of $28 \mathrm{~nm}$ and consists of 10 layers (alternating carbon and stainless steel). The roughness $\sigma_{R}$ of each layer was fitted using a diffusion profile with $\sigma_{R} \approx 2 \mathrm{~nm}$. The repetition rate of the superstructure is $N=12$. As a consequence of this, the quality of the multilayer derived by XRR is very poor, but a tendency can be found regarding the RBS and TEM results. 
The 60:40mod sample showed a verisimilar multilayer structure compared to the 50:50mod sample, but the diffusion of carbon and stainless steel was even higher. The RBS pattern of the 80:20 sample (which was fabricated with the 90:10 target with a reduced laser scan area and, thus, an increased scan frequency) showed a superstructure with similar measures, as well. As a consequence it is reasonable to assume, that the increase of the scan frequency induces a self-organizing effect, which leads to this superstructures. This is investigated more exactly in the next section.

As mentioned above, all samples show a decrease of the layer thickness during preceding deposition. As a result, a wobbler system of the target holder was developed. This system allows a controlled wobble of the target to prevent the cavation of the target. Here a wobble-angle of $\omega_{w o b}=3^{\circ}$ from the rest position was chosen. Under this conditions, the 50:50mod film was deposited, which is labeled in the following as 50:50modwob.

Fig. 7.38 shows the RBS result of the $50: 50$ modwob film.

In contrast to the other 50:50 films, the 50:50mod-
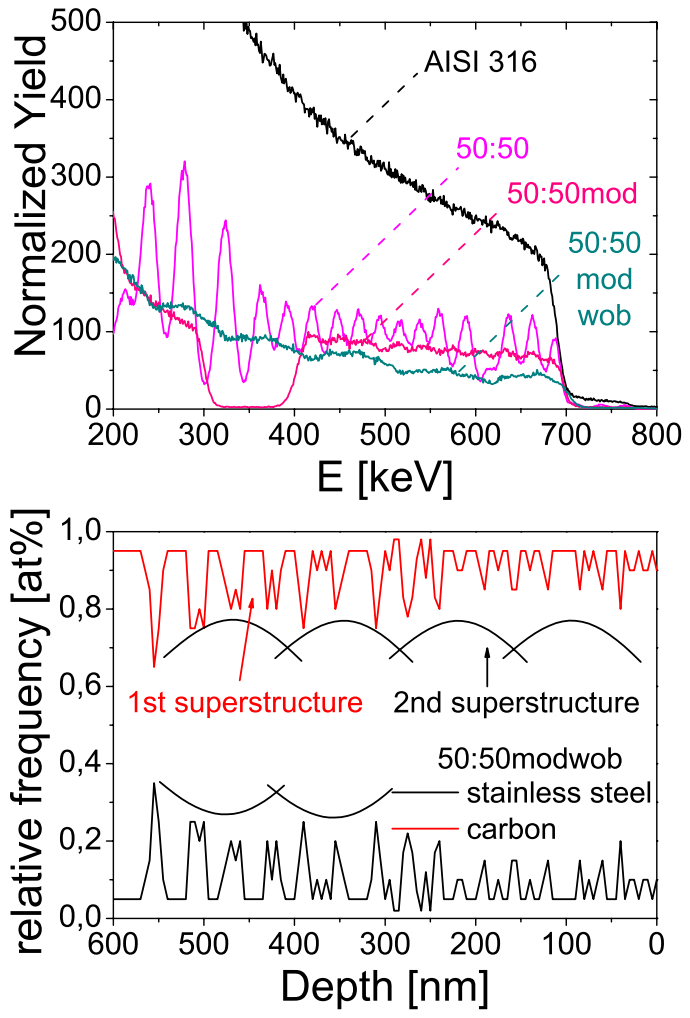

Figure 7.38: RBS spectra of the 50:50, 50:50mod and 50:50modwob STPLD film deposited at $298 \mathrm{~K}$. The depth profile of the 50:50modwob STPLD film can be found at the bottom.

wob film exhibit a broad oscillation in the RBS spectrum. On closer inspection, more small oscillations can be seen on the broad ones. In fact, the depth profile of the 50:50modwob film exhibits two superstructures: a small one, which was already seen in modified samples with a thickness of $25 \mathrm{~nm}$, and a new one with a thickness of 120-130 nm denoted by the semicircle in the depth profile. Since the 50:50modwob depth profile has the form of the depth profile of the post vacuum annealed 90:10 sample, a pry opened multilayer structure is expected. Unfortunately, the TEM patterns of this sample were not available until completion of this work and, thus, no further remarks can be done.

The properly aim to synthesize uniform multilayer structures has failed. Thus, the cavation of the target and the resulting shift of the laser plasma plume should be prevented by reducing the energy density of the laser (reducing via Q-switch or by increasing the target-substrate distance).

\subsubsection{Self-organization and nucle- ation model of carbon-stainless steel multilayer films}

The RBS spectra of all multilayer structured STPLD films indicated a self-organization of the films given that the number of multilayer as derived by TEM did not correspond to the laser scan process of the target.

Therefore, an EDX line scan of the 90:10 and 50:50mod STPLD films were carried out. The curve progression was the corrected by the Cliff-Lorimer equation [73]:

$$
I_{A}=C_{A} \cdot k_{A}
$$

where $I_{A}$ is the number of X-rays generated for, $C_{A}$ the concentrations and $k_{A}$ the Cliff-Lorimer sensitivity k-factor for element $A$.

Note: as a consequence of the insufficient sensitivity of the EDX detector to elements lighter than oxygen, no relative frequency could be derived (caused by the inappropriate carbon $\mathrm{k}$-factor).

Thereafter, the result was correlated with a time to space diagram of the scan process convoluted with the laser spot diameter $(\varnothing=2 \mathrm{~mm})$. Fig. 7.39 illustrates this for the 90:10 STPLD film.

At first glance, no correspondence between layer thickness and locality of the laser beam can be made. Although, the maximum of the carbon layers correspond to the moment when the laser spot hits the graphite inlay of the target, but the thickness does not comply.

As recently as the convolution of the laser beam on the time to space correlation (TTSC) is taken into account, a weak signal of a superstructure can be 


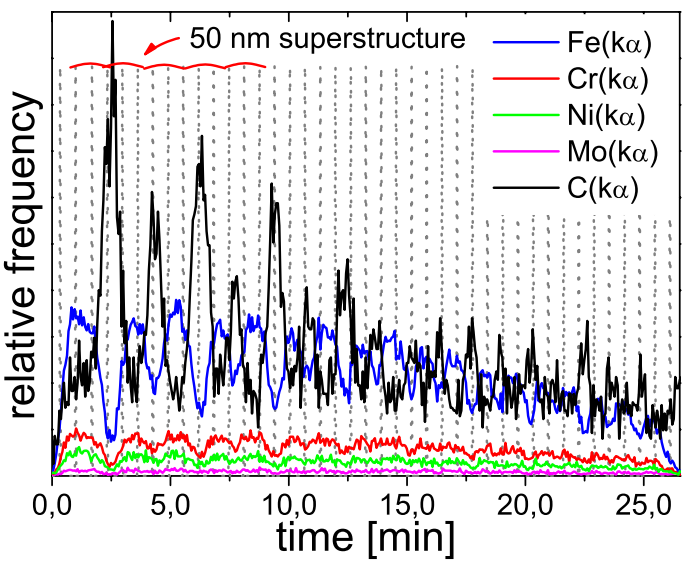

Figure 7.39: EDX-time to space correlation diagram of the 90:10 STPLD film. The dashed line corresponds to the situation, in which the laser spot hits the graphite layer of the target.

seen (as indicated by the red semicircles). This superstructure corresponds very well to the observed TEM multilayer structure. As a first summary, it is reasonable to assume, that the phenomenon observed in carbon - stainless steel multilayers is an induced self-organization: the alternating scan process of the target induces long range diffusion, which leads to segregation of carbon and the metal atoms, instead of forming carbides. As for inert sputtered stainless steel films, also here the Mo atoms can redistribute and induce long-range diffusion.

Note: if the observed phenomenon is based on a target scan effect, the multilayer structure should correspond to the distance/time between two dashed lines in the EDX TTSC diagram and

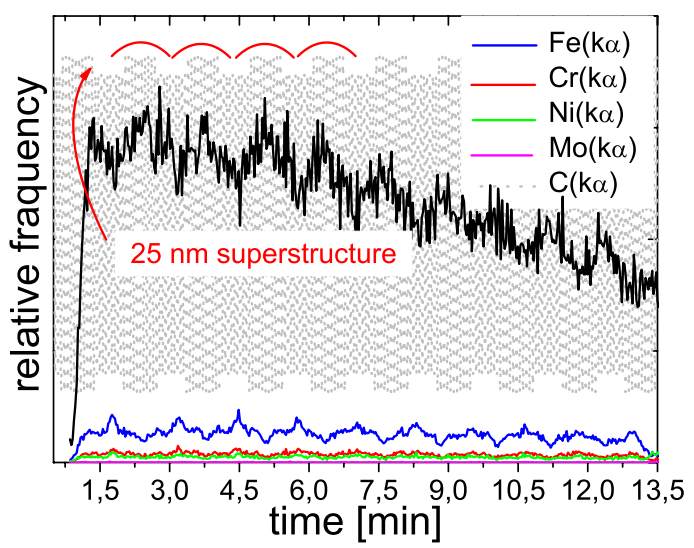

Figure 7.40: EDX-time to space correlation diagram of the 50:50 STPLD film. The dashed line corresponds to the situation, in which the laser spot hits the graphite layer of the target. the carbon layer should be very small (a few nanometers). This is not the case.

Fig. 7.40 shows the EDX TTSC diagram for the 50:50mod film.

Just as seen for the 90:10 EDX TTSC, a superstructure due to the convolution of laser beam and TTSC can be seen. Its thickness corresponds very well to the XRR, RBS and TEM results and is about $25 \mathrm{~nm}$. The number of dashed lines is significant higher than the number of multilayers caused by an exclusive scanning effect. As a consequence, the self-organization is also induced by the scanning of the target.

Nucleation model of carbon-stainless steel multilayer films Now the question arise, on which nucleation model are the observed multilayer structures based on?

As indicated by the TTSC diagrams, the selforganization in carbon - stainless steel multilayer is induced. First, the nucleation of the 90:10 film is discussed. Therefore, an estimation of the plasma ion energies is needed: by mapping the target voltage during deposition, an estimation of the plasma ion energies of the ablated target atoms can be obtained by taking the first derivative of the curve, which is shown for the 90:10 target in Fig. 7.41.

As a consequence, the adatoms have barely suf-

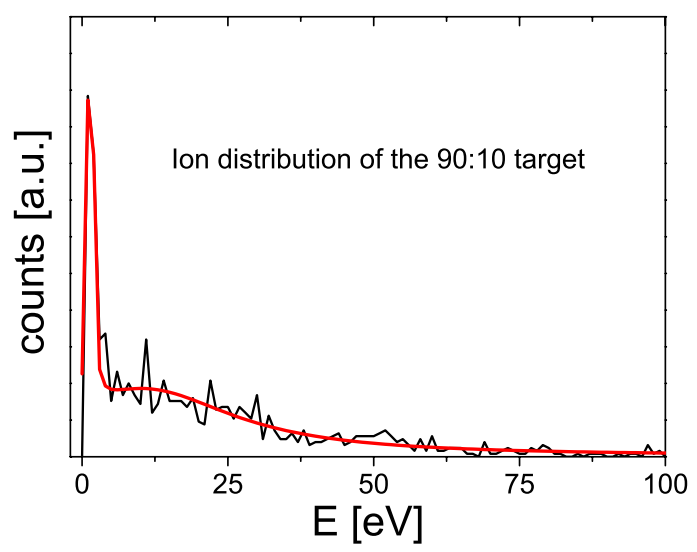

Figure 7.41: Ion distribution of the plasma ions of the 90:10 target.

ficient energy to adsorbate. By the reduction of energy, the influence of the Mo atoms seems to be more severe than in reactive sputtered films. No solidification occurs and benefits the carbon diffusion in the metal matrix. Local carbon inhomogeneities were induced via the target scan process. This induces local diffusion gradients which leads to an self-organizing effect and which promotes the decomposition of carbon and stainless steel and assists multilayer formation. 
Fig. 7.42 illustrates the plasma ion distribution of the 50:50mod target.

In contrast to the plasma ions of the 90:10 target,

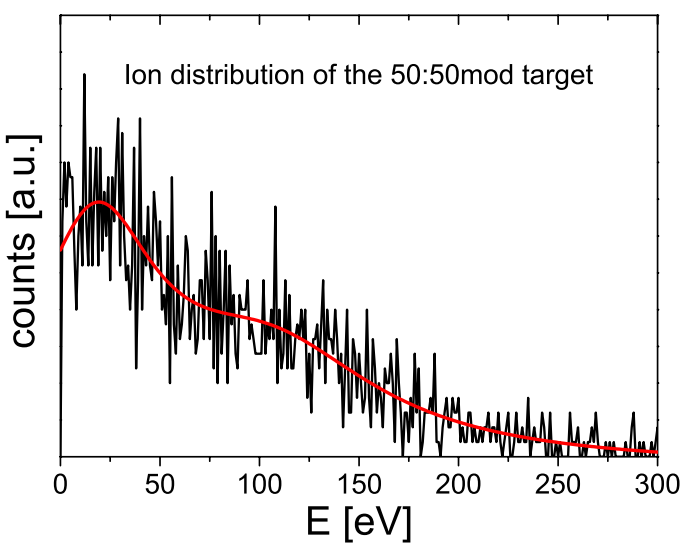

Figure 7.42: Ion distribution of the plasma ions of the 50:50 target.

the plasma ions of the 50:50mod target show considerably higher energies. As a result the nucleation gets more complicated given that ion implantation has to be taken into account.

In this case, the beginning of the nucleation is quite the same: Mo triggers a long-range diffusion and allows a carbon diffusion through the metal matrix. Via a SRIM simulation [95] an implantation depth of carbon into stainless steel (and vise versa) of about $3 \mathrm{~nm}$ is found. This implantation leads to a locally rearrangement which hinders the overall carbon diffusion in the matrix, but not complete. As a consequence the size of the superstructure is reduced. The ion implantation could lead to opposing diffusion gradients in the nano-scale regime, which could lead again to a self-organization and the formation of multilayer by decomposing carbon and stainless steel.

As mentioned above, all films show a decrease of the multilayer thickness during deposition process, which is caused by the cavation of the target. As a consequence, the laser plasma plume shifts and the deposition rate drops during deposition. Thus, no linear deposition rate can be assumed as done in the beginning. In fact, the deposition rate is higher than the calculated value and drops exponentially. The calculated value only constitutes a median value.

Finally, the formation of carbon - stainless steel multilayer with high-carbon concentrations were synthesized and crucial parameters, such as graphite-inlay thickness and, thus, the scan speed and substrate temperature determined. Now the question arise, if this procedure can be transferred to other elements and in which way the multilayer thickness is influenced for different elements. For this, experiments with pre-combined $\mathrm{Al}$ and $\mathrm{Ti}$ STPLD targets were performed.

The RBS results for the $\mathrm{Al} / \mathrm{C} 60: 40 \bmod$ film is depicted in Fig. 7.43.

Also here, a multilayer structure can be seen. How-
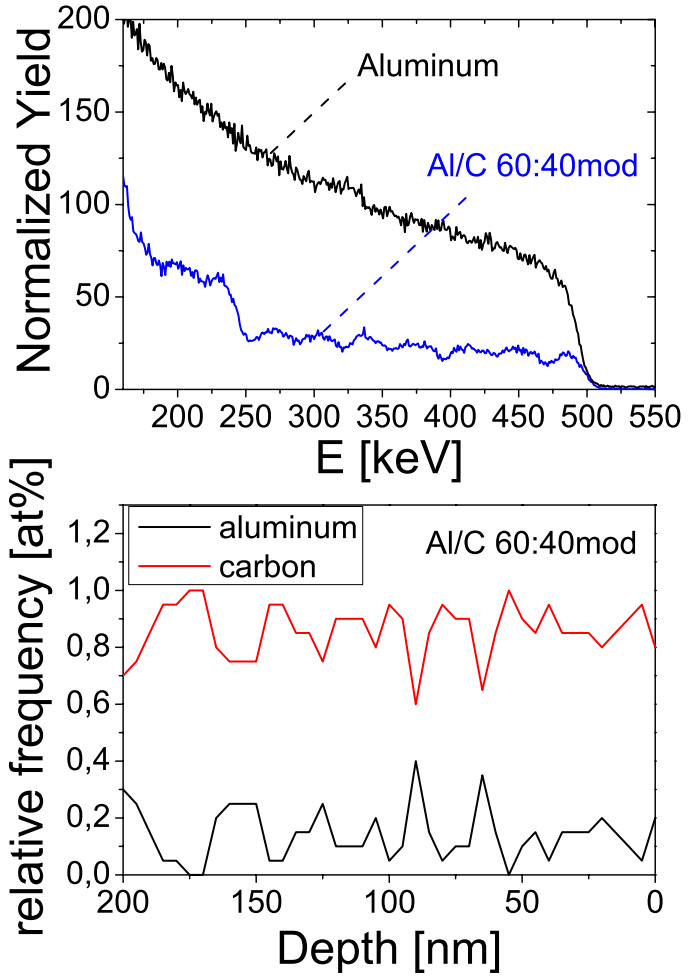

Figure 7.43: RBS spectra of the Aluminum bulk material and the Al/C 60:40mod STPLD film deposited at $298 \mathrm{~K}$. The depth profile of the $\mathrm{Al} / \mathrm{C}$ 60:40mod STPLD film can be found at the bottom.

ever, the depth profile exhibit pry opened multilayer structure, which is very surprising. Since it is known that carbon is not very solvable in $\mathrm{Al}$, a well defined structure was expected. On the other hand, the depth profile is not very reliable given that for film thicknesses, as observed here, marginal energies were achieved, in which the resolution is influenced by effects like straggling etc.

Nevertheless, a layer thickness of $\mathrm{Al}$ could be derived to about $15 \mathrm{~nm}$, whereas the carbon layer are only $5 \mathrm{~nm}$ in thickness. In comparison to carbon - stainless steel multilayer, the thickness has decreased. This is another hint for self-organization. If the formation is only based on a scan effect, for all elements the same multilayer structure would be expected given that the multilayer structure would only depend on the preparation of the target (and all targets were pre-combined in the same way).

Fig. 7.44 shows the RBS results for Ti multilayer. 
In comparison to all multilayer films prepared from
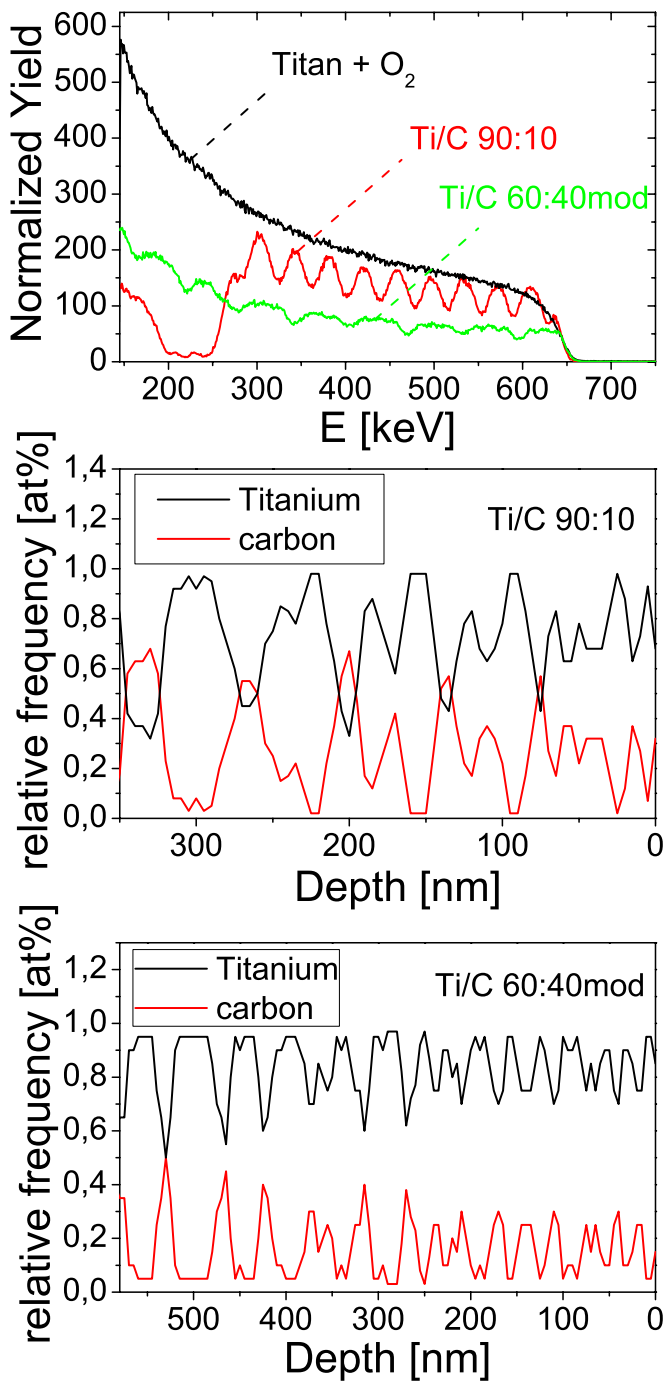

Figure 7.44: RBS spectra of the Titanium bulk material, the Ti/C 90:10 and the Ti/C 60:40mod STPLD films deposited at $298 \mathrm{~K}$. The depth profile of the $\mathrm{Ti} / \mathrm{C} 90: 10$ and of the $\mathrm{Ti} / \mathrm{C} 60: 40 \mathrm{mod}$ STPLD film can be found at the middle/bottom.

STPLD targets, Ti/C multilayer exhibit the clearest RBS oscillations indicating a well defined multilayer structure for films deposited at room temperature. In deed, this is confirmed by the depth profiles. A layer thickness of Ti could be derived to about $20 \mathrm{~nm}$, whereas the carbon layer are only $10 \mathrm{~nm}$ in thickness - still being smaller than in carbon - stainless steel multilayer.

Fig. 7.45 shows the dependence between multilayer thickness and atomic number at constant carbon content. For this, all films, which were prepared by the same STPLD target configuration were summarized, viz. SS/C-, Al/C- and Ti/C 60:40mod films. A clear tendency can be seen, where the layer thick-

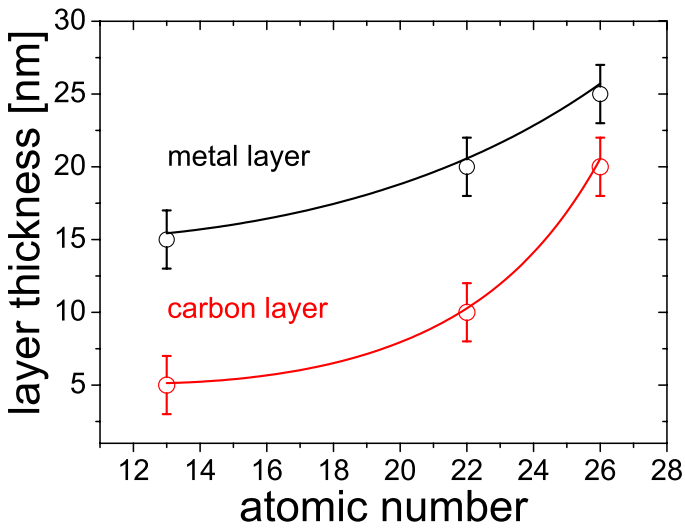

Figure 7.45: Dependence between multilayer thickness and atomic number at constant carbon content. Here, the thicknesses of SS/C, Al/C and Ti/C 60:40mod samples are summarized.

ness - both metal and carbon - is increasing exponentially with increasing atomic number. Even these would not be expected, if the multilayer formation were only based on a target scanning effect (it should be constant). This indicates again a selforganizing effect.

By the introduction of the STPLD technique, a new induced self-organizing effect was found. First experiments showed crucial processing parameters, but more experiments have to be done, to understand the complete process and to achieve films, which can be used for industrial application. Therefore, experiments with varying laser energy should be carried out to synthesize multilayer films, which layer interfaces are sharper (e.g. to use those films in the electronic industry as conducting paths or for X-ray mirrors by achieving sufficient crystallinity). Furthermore, a wide range of elements should be used to fabricate STPLD films to investigate the evolution of multilayer thickness to obtain an empirical formula for this phenomenon.

As next step, the STPLD method is applied to other inlays, e.g. boron-nitride, to investigate, if multilayer systems can be formed with other materials and if amorphous films can be obtained. The next section will discuss those results.

\subsection{Results for boron - im- planted and boro - ni- trided stainless steel films}

The films were prepared by different methods: (1) conventional stainless steel samples (X5CrNiMo17$12-2,1.4401$; size $10 \times 10 \mathrm{~mm}$ ) containing 1,3 and 
6 at.\% boron were prepared by ion implantation at the IONAS accelerator facility in Göttingen. Using SRIM [95], fluences for different energies were calculated, to form implantation profiles with the desired B contents. The parameters are summarized in Table 7.14. The implantation profiles are

Table 7.14: Implantation fluences for stainless steel samples containing 1, 3 and 6 at.\% for different implantation energies. The samples are labeled as $1 \mathrm{P}$, $3 \mathrm{P}$ and $6 \mathrm{P}$

\begin{tabular}{cccc}
$\begin{array}{c}\mathrm{E} \\
{[\mathrm{keV}]}\end{array}$ & $1 \mathrm{P}$ & $3 \mathrm{P}$ & $6 \mathrm{P}$ \\
\hline 200 & $1.2 \cdot 10^{16}$ & $4.0 \cdot 10^{16}$ & $8.0 \cdot 10^{16}$ \\
130 & $7.5 \cdot 10^{15}$ & $2.0 \cdot 10^{16}$ & $3.8 \cdot 10^{16}$ \\
65 & $6.5 \cdot 10^{15}$ & $2.0 \cdot 10^{16}$ & $4.0 \cdot 10^{16}$ \\
30 & $3.2 \cdot 10^{15}$ & $8.5 \cdot 10^{15}$ & $1.5 \cdot 10^{16}$ \\
15 & $8.5 \cdot 10^{14}$ & $4.0 \cdot 10^{15}$ & $8.5 \cdot 10^{15}$ \\
\hline
\end{tabular}
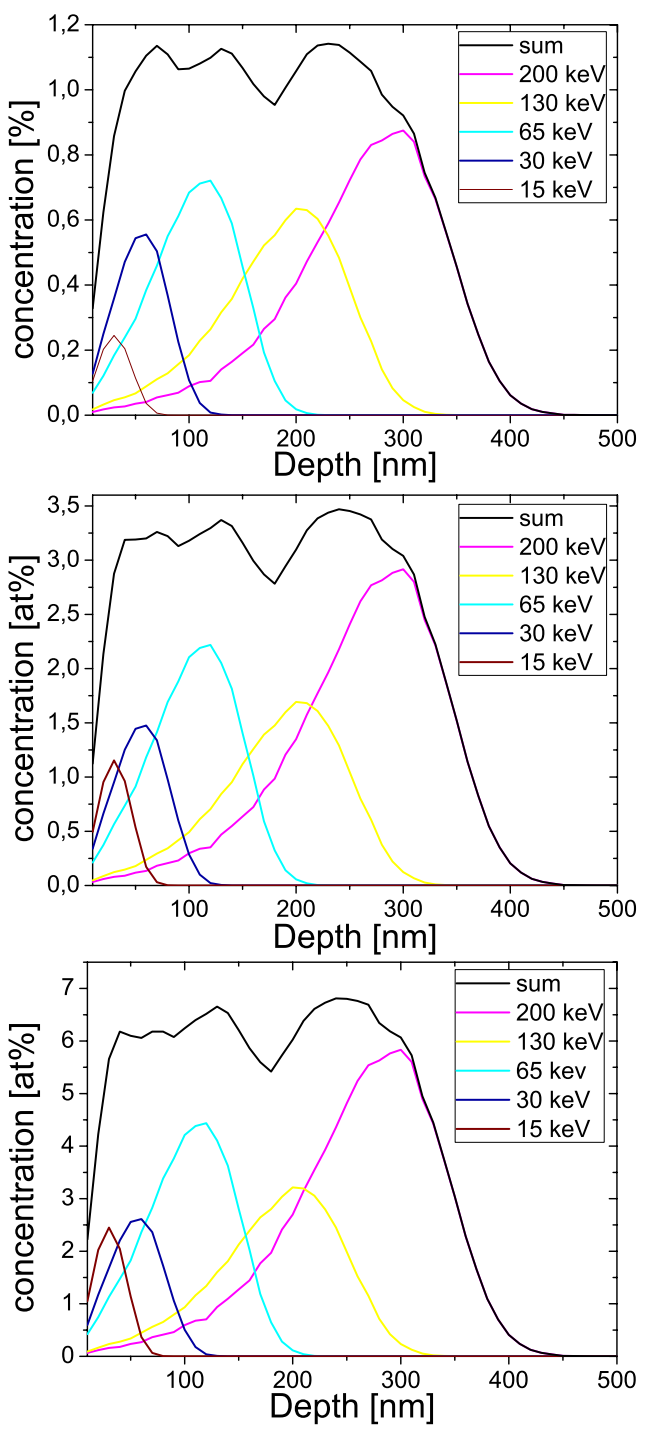

Figure 7.46: Implantation profiles calculated by SRIM for stainless steel samples containng 1, 3 and 6 at.\% boron.

shown in Fig. 7.46.

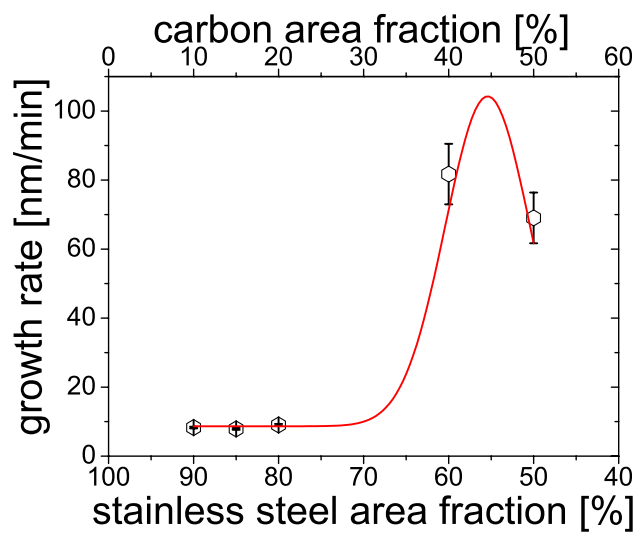

Figure 7.47: Growth rate $g$ as a function of the boron-nitride area fraction for the room temperature STPLD deposition.

(2) Films were deposited by Sequential Target Pulsed Laser Deposition (STPLD) with a Brilliant Nd:YAG laser $(\lambda=1064 \mathrm{~nm}$, pulse duration 5-6 ns, repetition rate $20 \mathrm{~Hz}$ ) onto amorphous $\mathrm{SiO}_{2}$ substrates (oxidized $\mathrm{Si}(100)$ wafer of $0.5 \mathrm{~mm}$ thickness, pre-cleaned with acetone and oxidized in air, no further treatment) utilizing a commercial AISI 316 (X5CrNiMo17-12-2, 1.4401) target. Before deposition, the PLD-chamber was evacuated to a base pressure of $10^{-6} \mathrm{~Pa}$. The laser energy was set to $5 \mathrm{~mJ} / \mathrm{cm}^{2}$. The targets were pre-combined in that way, that area ratios (of stainless steel to boron nitride) of 90:10, 85:15, 80:20, 60:40mod and 50:50mod were obtained (laser scan area $20 \times 2 \mathrm{~mm}$ on a $25 \times 25 \mathrm{~mm}$ STPLD target - excerpt the 80:20 sample: here the 90:10 STPLD target was used, the desired area ratio was obtained by reducing the laser scan area to $10 \times 2 \mathrm{~mm}$ ). The thickness of the deposited films was controlled by a quartz microbalance (with a film density set to $7.89 \mathrm{~kg} / \mathrm{m}^{3}$, which is the density of AISI 316). The deposition parameters and the resulting thicknesses and growth rates are summarized in Table 7.15.

From these data it is derived that the growth

Table 7.15: Deposition time $t$, real film thickness $d$ (as measured by RBS after deposition) and derived growth rate $g=\frac{d}{t}$ for the STPLD films. All samples were deposited at $298 \mathrm{~K}$ with a laser energy of $5 \mathrm{~mJ} / \mathrm{cm}^{2}$ and a target-substrate distance of $6.5 \mathrm{~cm}$.

\begin{tabular}{cccc}
\hline sample & $\begin{array}{c}t \\
{[\mathrm{~min}]}\end{array}$ & $\begin{array}{c}d \\
{[\mathrm{~nm}]}\end{array}$ & $\begin{array}{c}g \\
{[\mathrm{~nm} / \mathrm{min}]}\end{array}$ \\
\hline SSB90:10 & $39: 15$ & $325(5)$ & $8.28(38)$ \\
SSB85:15 & $36: 07$ & $285(5)$ & $7.89(52)$ \\
SSB80:20 & $35: 59$ & $244(5)$ & $9.03(55)$ \\
SSB60:40mod & $10: 43$ & $876(5)$ & $81.74(877)$ \\
SSB50:50mod & $10: 38$ & $734(5)$ & $69.03(735)$ \\
\hline
\end{tabular}


rate has a Gaussian form with increasing carbon area fraction, which seems to be characteristic for STPLD processes. The correlation between grothrate $g$ and inlay fraction is visualized in Fig. 7.19.

\subsubsection{Results for the implanted steel samples}

To confirm the calculated boron content, RBS measurements were performed on the implanted stainless steel samples. The results are shown in Fig.7.49.

As expected, the RBS spectra show exactly the

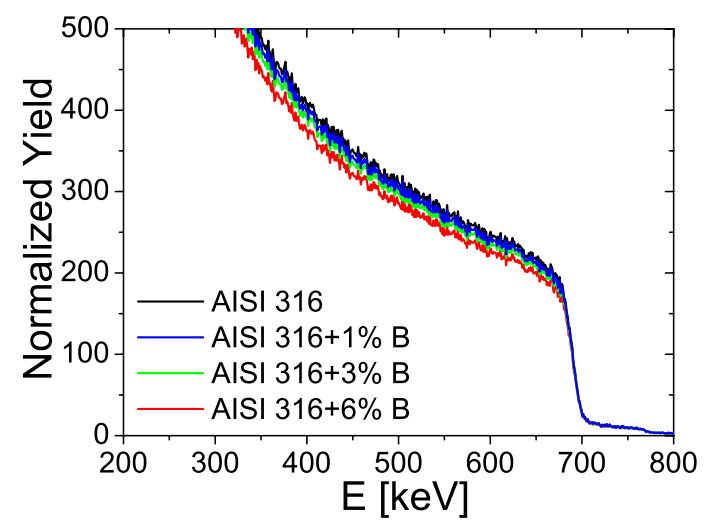

Figure 7.48: RBS spectra of AISI 316 samples implanted with 1, 3 and 6 at.\% boron.

calculated boron content.

XRD shows well defined reflexes. Three different phases can be seen in all samples: the $\gamma-(\mathrm{Fe}, \mathrm{Cr}, \mathrm{Ni})$ phase - remnants from the original starting material, the $\alpha-(\mathrm{Fe}, \mathrm{Cr}, \mathrm{Ni})$ phase - typically observed after ion implantation as a result of ion mixing, and the $(\mathrm{Fe}, \mathrm{Cr}, \mathrm{Ni})_{3} \mathrm{~B}$. To derive the $(\mathrm{Fe}, \mathrm{Cr}, \mathrm{Ni})_{3} \mathrm{~B}$ content, a peak analysis was performed. For clarity, only the pattern and peak analysis of the $6 \mathrm{P}$ sample is shown.

Also here, the observed area fractions correspond to the implanted boron contents.

The CEM spectra are summarized in Fig. 7.51.

The spectra reveal both magnetically split sextets and paramagnetic sites. The fitted distributions are in good agreement to the XRD measurements. The hyperfine field distribution consist of two components, one corresponding to $\alpha$-Fe with a random neighborhood (consisting of $\mathrm{Cr}, \mathrm{Ni}$ and $\mathrm{Mo}$ ) and one component which could be attributed to $(\mathrm{Fe}, \mathrm{Cr}, \mathrm{Ni})_{2} \mathrm{~B}$ or $(\mathrm{Fe}, \mathrm{Cr}, \mathrm{Ni})_{3} \mathrm{~B}$ borides [147]. The quadrupole splittings also confirm the XRD results, which exhibit a component corresponding to the original target material, and one which corresponds to the formation of austenite. The results of the
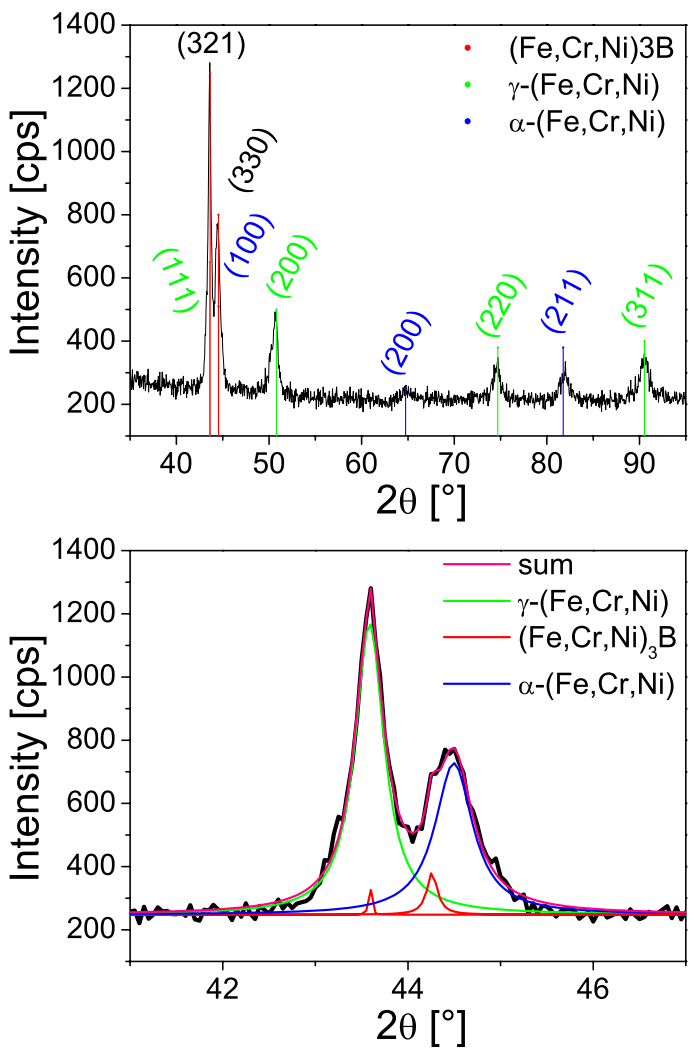

Figure 7.49: GIXRD $\left(2^{\circ}\right)$ spectrum and peak analysis of the AISI 316 sample implanted with 6 at.\% boron.
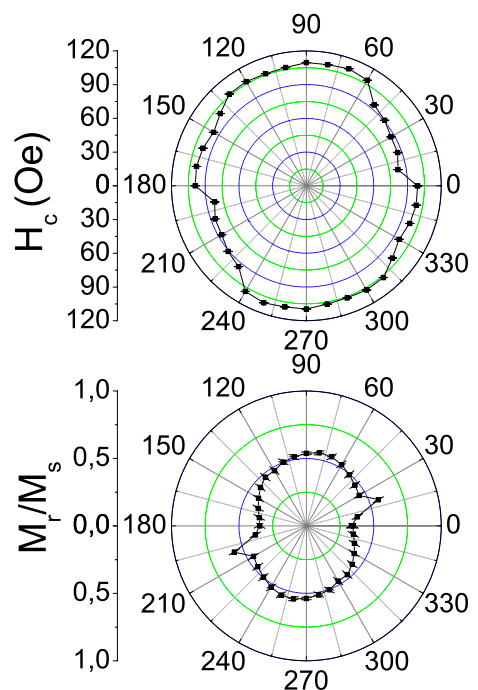

Figure 7.50: Polar diagrams of the coercive field $H_{\mathrm{C}}$ (top) and the relative remanence $M_{\mathrm{R}} / M_{\mathrm{S}}$ (bottom) of AISI 316 sample implanted with 1 at.\% boron.

fitting procedures are presented in Table 7.16. These Mössbauer results were confirmed by MOKE 

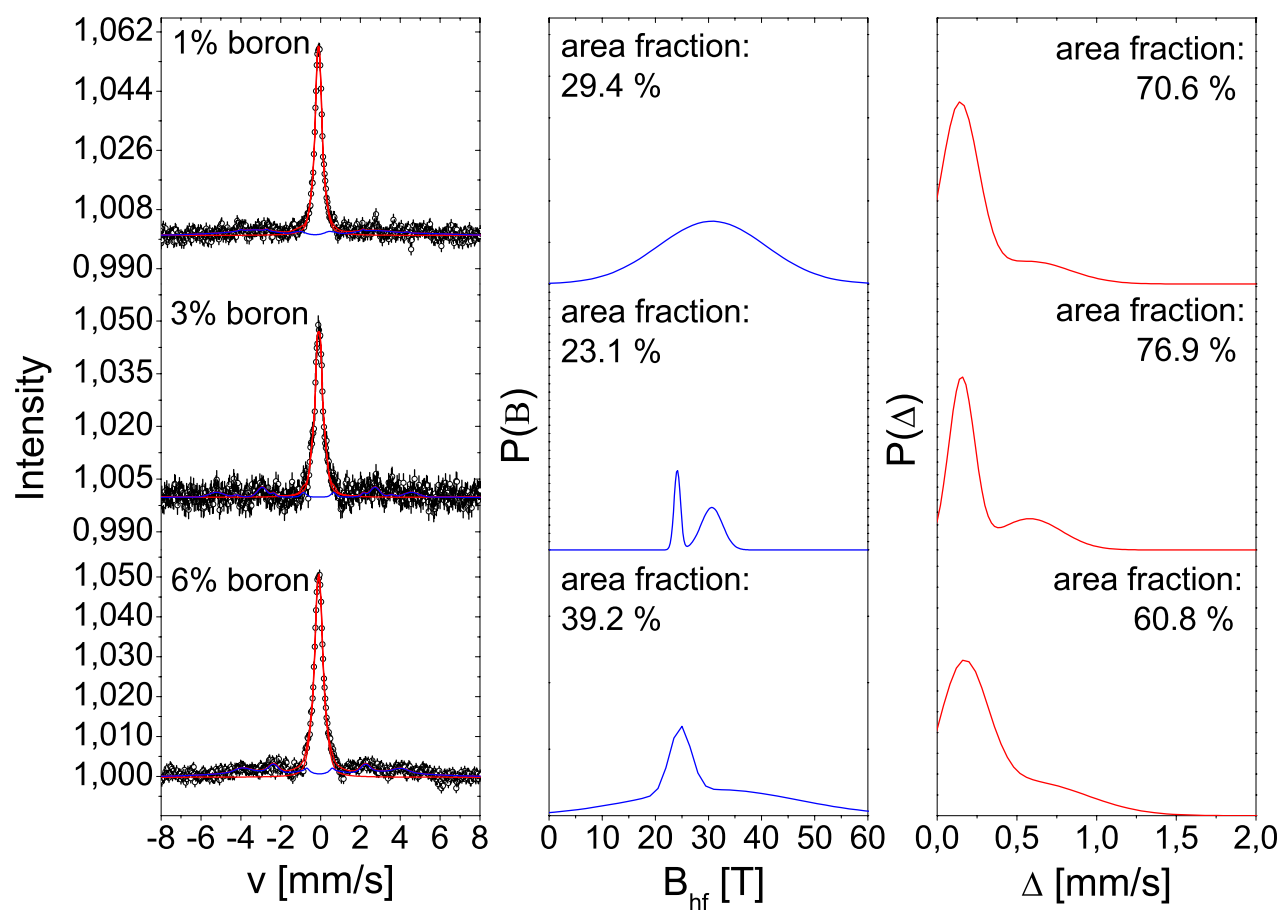

Figure 7.51: Mössbauer spectra of the implanted AISI 316 films. The numbers in the graphs represent the implanted boron concentration.

measurements. Figure 7.50 shows the result of the MOKE measurement of the AISI 316 sample containing 1 at.\% boron. The other samples show similar MOKE measurements.

No clear anisotropy can be identified, but it seems to be a mixture of a uniaxial and a fourfold anisotropy indicating phase transformations form $\alpha$ - to $\gamma$-phase and vise versa. The uniaxial anisotropy is usually seen after ion implantations. This behavior could also be due to magnetostriction effects by stresses in the film. The coercive field $H_{C}$ of all samples is approximately 80 Oe.

Summarizing, no amorphization of the boron implanted stainless steel samples was obtained, but rather a crystallization of borides due to the implantation of boron and a phase transformation from $\gamma$ to $\alpha$-phase due to mixing effects during implantation. As next step, higher boron contents will be incorporated by the STPLD technique. The results are presented in the next section.

\subsubsection{Results for boro-nitrided stain- less steel films}

Deposition at room temperature and influence of the boron-nitride area fraction The inlay area, and thus the carbon concentration, was systematically increased from 10 to $50 \%$. For all

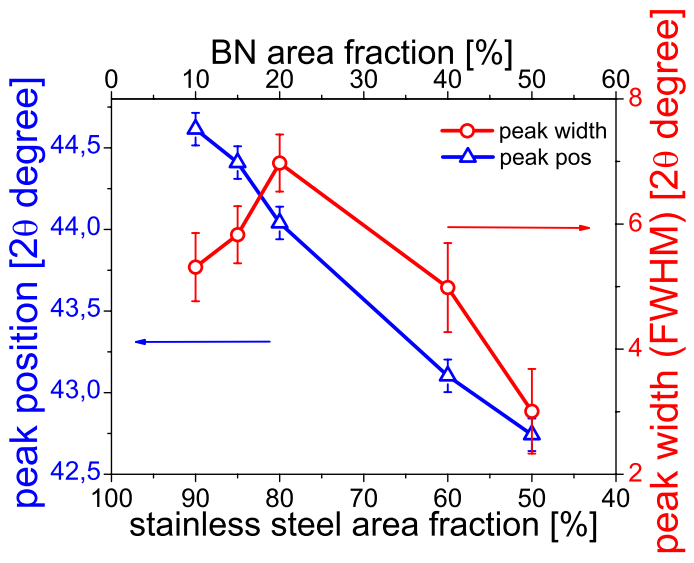

Figure 7.52: Peak position and peak width of the first broad peak in the XRD spectra of the STPLD films.

these deposited films, the STPLD samples show the mostly broad XRD appearance of amorphous materials, but also a reflex corresponding to boronnitride can be seen indicating a crystallization of BN-grains. The XRD spectra are resembled in Fig. 7.53. Fig. 7.52 shows the position and the width of the first broad peak for the spectra shown in Fig. 7.53. There is a clear tendency for peak position and peak width, where the peak position 
Table 7.16: Mössbauer fitting results of the boron implanted AISI 316 samples, deposited at room temperature ( $f$ - area fraction (error), mean values of $\langle\delta\rangle$ - isomer shift, $\langle\Delta\rangle$ - the quadrupole splitting for the paramagnetic subspectra, $\langle\epsilon\rangle$ the quadrupole splitting for the magnetic subspectra, $B$ - hyperfine field).

\begin{tabular}{ccrrrrrr}
\hline sample & part & $\begin{array}{r}f \\
{[\%]}\end{array}$ & $\begin{array}{r}\langle\delta\rangle \\
{[\mathrm{mm} / \mathrm{s}]}\end{array}$ & $\begin{array}{r}\langle\Delta\rangle,\langle\epsilon\rangle \\
{[\mathrm{mm} / \mathrm{s}]}\end{array}$ & $\begin{array}{r}\sigma_{\Delta} \\
{[\mathrm{mm} / \mathrm{s}]}\end{array}$ & $\begin{array}{r}\langle B\rangle \\
{[\mathrm{T}]}\end{array}$ & $\begin{array}{r}\sigma_{B} \\
{[\mathrm{~T}]}\end{array}$ \\
\hline $1 \mathrm{P}$ & ferro-1 & $1.2(5)$ & -0.05 & 0.03 & & $25.8(6)$ & $1.1(9)$ \\
& ferro-h & $28.2(12)$ & -0.05 & 0.03 & & $30.7(4)$ & $10.1(44)$ \\
& para1 & $55.4(32)$ & -0.09 & 0.14 & 0.12 & & \\
& para2 & $15.2(9)$ & 0.10 & 0.58 & 0.25 & & \\
\hline $3 \mathrm{P}$ & ferro-1 & $3.5(3)$ & -0.02 & 0.10 & & $24.2(22)$ & $0.6(2)$ \\
& ferro-h & $19.6(15)$ & -0.02 & 0.10 & & $30.4(12)$ & $2.0(4)$ \\
& para1 & $52.9(55)$ & -0.11 & 0.15 & 0.08 & & \\
& para2 & $24.0(17)$ & 0.11 & 0.58 & 0.20 & & \\
\hline $6 \mathrm{P}$ & ferro-1 & $5.8(7)$ & 0.03 & 0.06 & & $24.7(74)$ & $2.0(9)$ \\
& ferro-h & $33.4(22)$ & 0.03 & 0.06 & & $31.5(12)$ & $15.3(48)$ \\
& para1 & $45.2(49)$ & -0.08 & 0.16 & 0.15 & & \\
& para2 & $15.6(11)$ & 0.13 & 0.58 & 0.34 & & \\
\hline
\end{tabular}

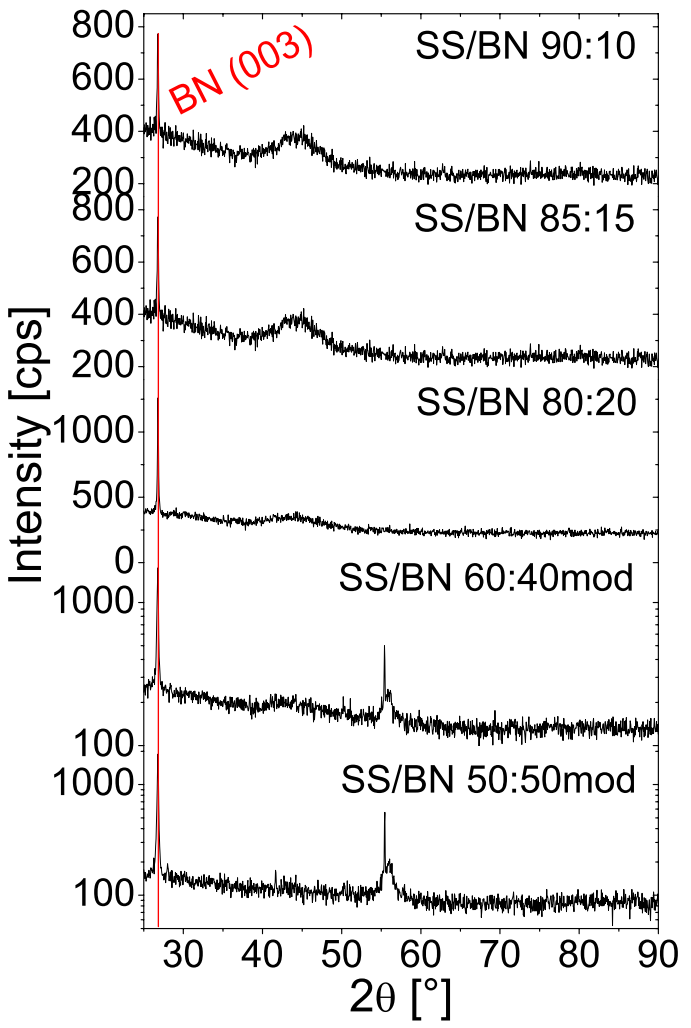

Figure 7.53: GIXRD $\left(2^{\circ}\right)$ spectra of the carburized films. The stainless steel - boron nitride area fractions are given in the graph.

is decreasing linear with increasing boron-nitride, whereas the peak width has a parabolic form indicating different phase transformations.

Fig. 7.54 illustrates the CEM spectra of the STPLD films, which show the transition from a phase and which is verisimilar to the amorphous and soft ferromagnetic phase observed in carburized, nitrided and oxidized stainless steel films. The only difference seems to be the absence of a component with higher hyperfine fields. The remaining component with small hyperfine fields - and the only one observed in boro-nitrided stainless steel films - can be assigned to the FeB phase [147]. Noticeable is, that the formation of the $\mathrm{FeB}$ phase needs a minimum boron content of $50 \%$. However, the films which show this amorphous phase are deposited from STPLD targets, which should deposit a maximum B content of $20 \%$. As a consequence, a boron segregation took place during deposition or areas have been locally formed with boron content of nearly/over $50 \%$. The films deposited from the SS/BN 60:40mod and SS/BN 50:50mod targets exhibit a doublet structure in their spectra. This could be attributed to a incorporation of more nitrogen out of the boron-nitride inlay of the target. Another possibility could be, that the observed doublet structure could be attributed to a $\mathrm{Fe}_{x} \mathrm{~B}_{100-x}$. As a fact, that the XRD patterns only exhibit a broad appearance (and thus, that of amorphous materials), no further remarks can be done and none of the two possibilities can be limited as more probable. The results of the fitting procedures can be found in Table 7.17.

Figure 7.55 shows the result of the MOKE measurement of the SS/BN 90:10 STPLD film. It exhibits only a fourfold anisotropy, which is normally observed after phase transformation from a bcc to fcc phase or vice versa $[89,98]$. The coercive field was derived to 66 Oe. Actually, a soft ferromagnetic phase was expected. This indicates, that the missing high-hyperfine field component in the Mössbauer spectra is attributed a spin glassy phase containing $\mathrm{Fe}$, which surrounds the found disordered $\mathrm{Ni}_{3} \mathrm{C}$ phase. All other STPLD samples exhibit a verisimilar behavior like the 90:10 film. 

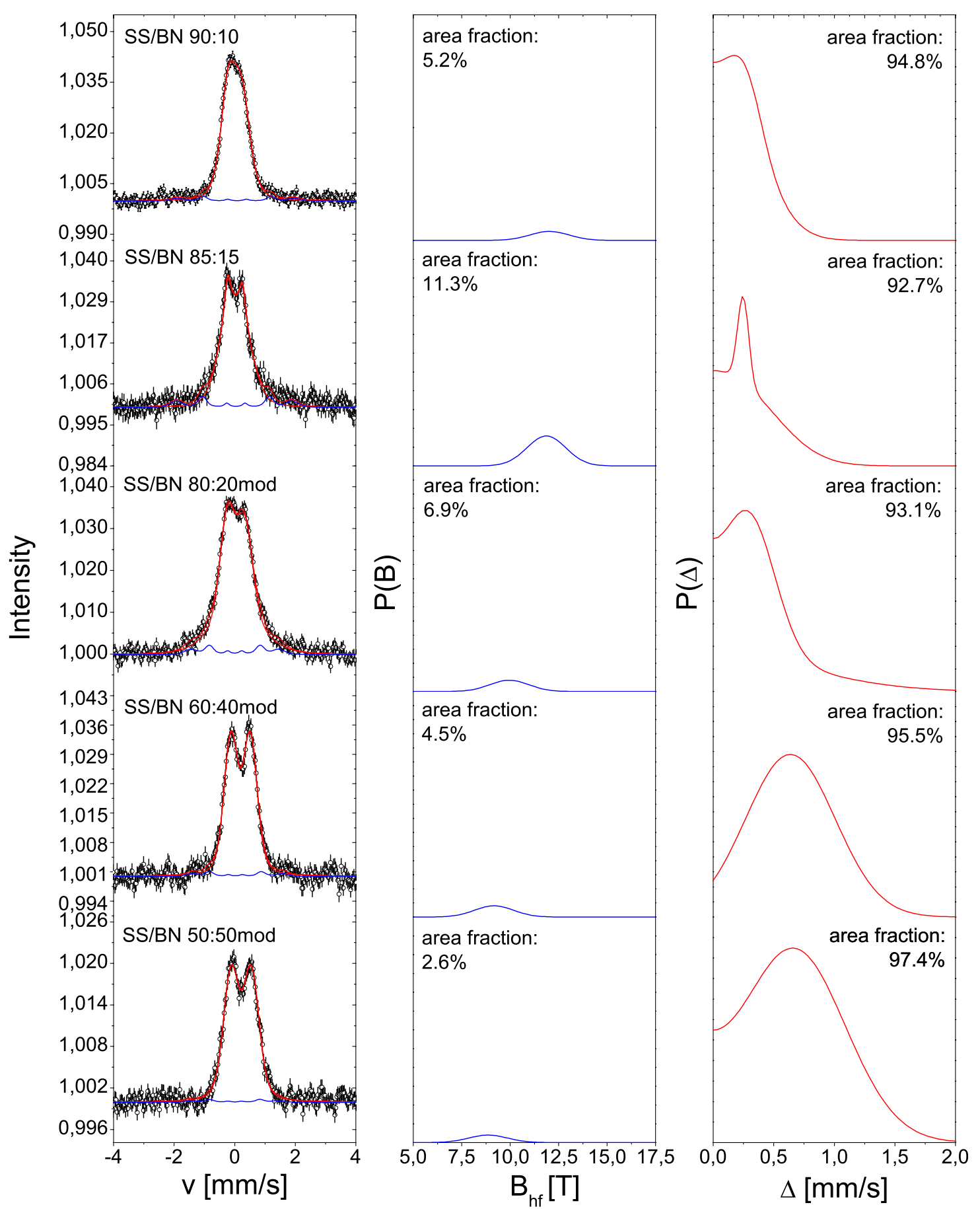

Figure 7.54: Mössbauer spectra of the STPLD stainless steel films. The numbers in the graphs represent the stainless steel/boron-nitride fraction of the STPLD targets. 


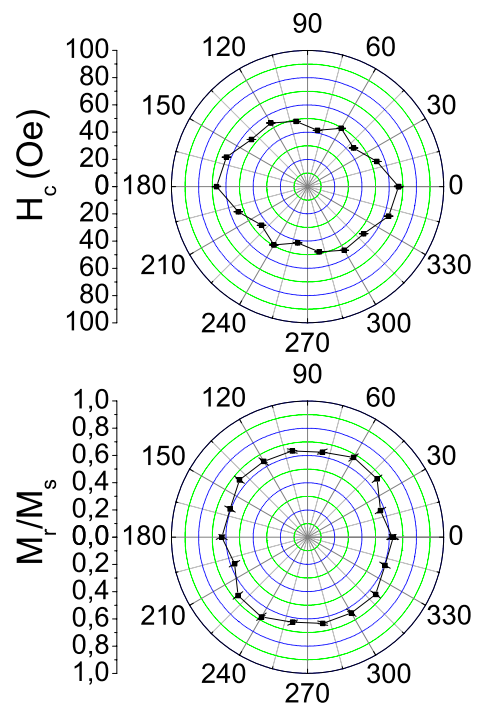

Figure 7.55: Polar diagrams of the coercive field $H_{\mathrm{C}}$ (top) and the relative remanence $M_{\mathrm{R}} / M_{\mathrm{S}}$ (bottom) of SS/BN 90:10 STPLD sample.

To evaluate the amount of incorporated boron and nitrogen, RBS measurements were carried out, whose results are shown in Fig. 7.56.

Due to the limited limited mass resolution of RBS,

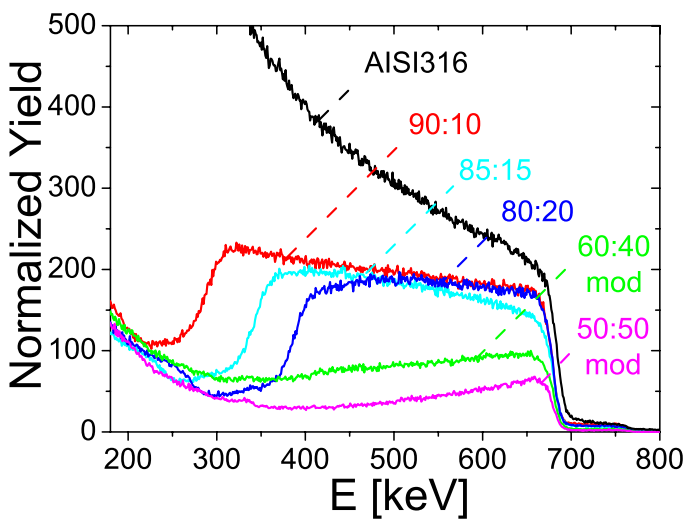

Figure 7.56: RBS spectra of the STPLD films. The stainless steel/boron-nitride area ratios are given in the graph.

elements lighter than oxygen can not be accurately determined. However, a trend can be pointed out. As seen in the CEM spectra, RBS exhibit a verisimilar boron and nitrogen content $(20 \%)$ in the 90:10, 85:15 and 80:20 STPLD films. The reduction of the yield of the STPLD films indicates a boron-nitride surface layer of a few nano-meters. Generally, more boron than nitrogen can be observed in all films and the hypothesis of locally enriched boron domains seems to be confirmed. However, more important is the fact, that no oscillations can be observed in the RBS spectra. This indicates that the films exhibit no multilayer structure.

Fig. 7.57 illustrates the TEM pattern of the SS/BN 80:20STPLD film.

Noticeable problems can be seen: 1) several BN

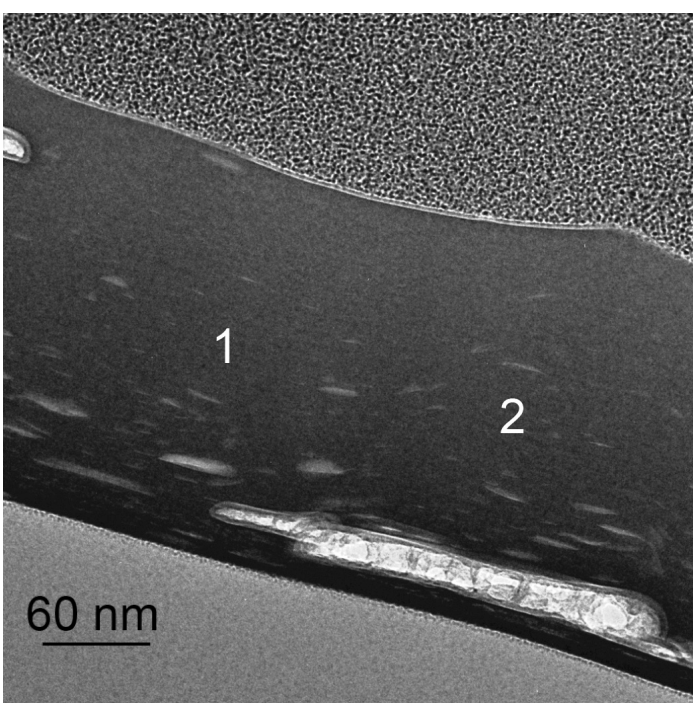

Figure 7.57: TEM pattern of the SS/BN 80:20 STPLD film. The two numbers in the pattern indicate the position, where the histograms were taken.

pieces are distributed through the film and 2) a weak multilayer structure can be observed. To confirm this, histograms were taken which are depicted in Fig. 7.58.

The histograms confirm a very weak multilayer structure, which is difficult to see in the TEM pattern due to the similarity of the compositions of the multilayer. The thickness of the "metal rich" layer is about $3 \mathrm{~nm}$ and $10 \mathrm{~nm}$ of the "metal poor" layer, respectively. Thus, the layer thickness can not be resolved by RBS.

Now the question arise, which mechanism hinders multilayer growth: possibly, there are two factors, which are hindering. The first factor could be the inlay material, the boron nitride. As described above, the STPLD process induces diffusion. Since the inlay material consists of two elements, competing diffusion processes could be induced, which are hindering multilayer growth. However, the more probable factor seems to be the BN droplets, which are distributed through the film. Droplet formation is due to hydrodynamic instabilities generated during laser-induced melting and ablation [45]. There are four different mechanisms, which lead to droplet formation: 1) the expansion velocity of the laser plasma plume induces surface capillary waves; 2) Rayleigh-Talyor instabilities, caused by multiplepulse irradiation, which lead to the motion of the 
Table 7.17: Mössbauer fitting results of the boron implanted AISI 316 samples, deposited at room temperature $(f$ - area fraction (error), mean values of $\langle\delta\rangle$ - isomer shift, $\langle\Delta\rangle$ - the quadrupole splitting for the paramagnetic subspectra, $\langle\epsilon\rangle$ the quadrupole splitting for the magnetic subspectra, $B$ - hyperfine field).

\begin{tabular}{ccrrrrrr}
\hline sample & part & $\begin{array}{r}f \\
{[\%]}\end{array}$ & $\begin{array}{r}\langle\delta\rangle \\
{[\mathrm{mm} / \mathrm{s}]}\end{array}$ & $\begin{array}{c}\langle\Delta\rangle,\langle\epsilon\rangle \\
{[\mathrm{mm} / \mathrm{s}]}\end{array}$ & $\begin{array}{r}\sigma_{\Delta} \\
{[\mathrm{mm} / \mathrm{s}]}\end{array}$ & $\begin{array}{r}\langle B\rangle \\
{[\mathrm{T}]}\end{array}$ & $\begin{array}{r}\sigma_{B} \\
{[\mathrm{~T}]}\end{array}$ \\
\hline \multirow{2}{*}{$90: 10$} & ferro & $5.2(15)$ & 0.05 & -0.02 & & $12.0(16)$ & $1.4(7)$ \\
& para1 & $75.8(22)$ & 0.01 & 0.34 & 0.56 & & \\
& para2 & $19.0(11)$ & 0.10 & 0.55 & 0.26 & & \\
\hline $85: 15$ & ferro & $11.3(2)$ & 0.01 & -0.03 & & $11.9(9)$ & $0.9(11)$ \\
& para1 & $74.5(11)$ & & 0.49 & 0.09 & & \\
& para2 & $14.2(4)$ & 0.02 & 0.55 & 0.69 & & \\
\hline $80: 20$ & ferro & $6.9(1)$ & 0.12 & 0.03 & & $10.0(8)$ & $1.0(2)$ \\
& para1 & $43.4(22)$ & 0.03 & 0.10 & 1.54 & & \\
& para2 & $49.7(14)$ & 0.03 & 0.58 & 0.43 & & \\
\hline $60: 40 \mathrm{mod}$ & ferro & $4.5(7)$ & 0.05 & 0.03 & & $9.2(19)$ & $1.1(9)$ \\
& para & $95.5(13)$ & 0.19 & 0.65 & 0.35 & & \\
\hline $50: 50 \mathrm{mod}$ & ferro & $2.6(1)$ & & & & $9.2(19)$ & $1.1(9)$ \\
& para & $97.4(13)$ & 0.21 & 0.67 & 0.42 & & \\
\hline
\end{tabular}
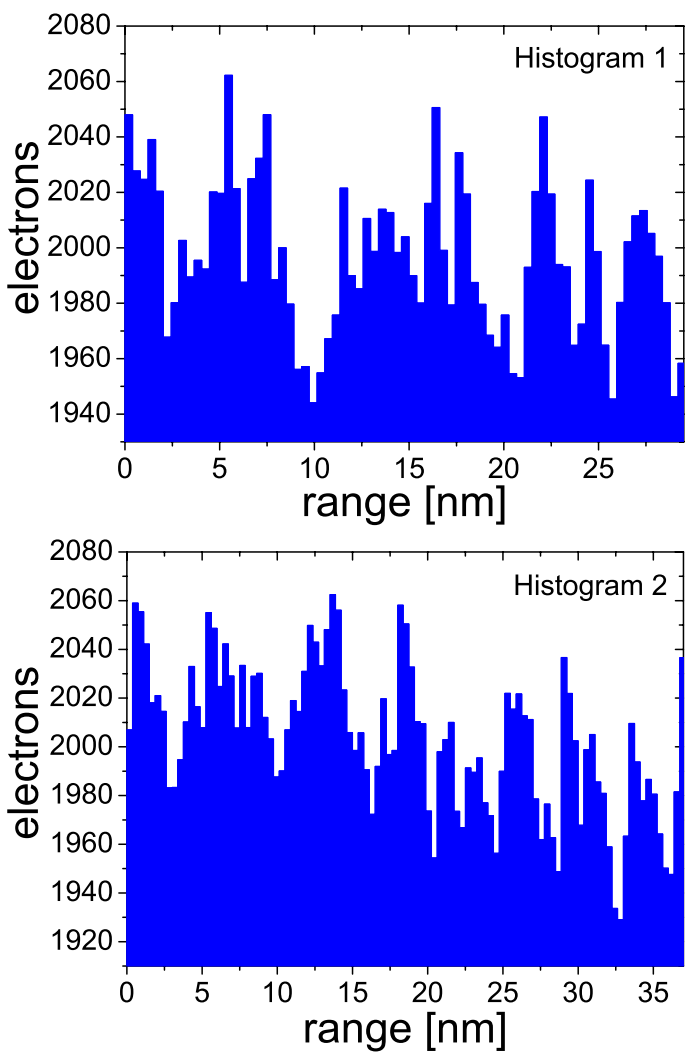

Figure 7.58: Histograms taken from the positions shown in the SS/bn 80:20 TEM pattern.

target liquid from valleys of capillary waves. This leads to an increase of corrugation and centrifugal forces near the hills cause droplet formation. 3) Kevin-Helmholtz (shear flow) instabilities and 4) Necking and formation of solid particulates [45]. Here, the second mechanism is more probable.
During multilayer growth, nucleation usually takes place on top of terraces where the geometry of the diffusion process is well defined [148]. Thus, droplet deposition prevents multilayer growth.

Summarizing, the boro-nitriding of stainless steel lead to a verisimilar amorphous phase, which was observed in carburized, nitrided and oxidized stainless steel films. The difference between these amorphous phases is found in magnetism. Whereas the amorphous phase in magnetron and RPLD deposited exhibits two magnetic components and is soft ferromagnetic, the boro-nitrided amorphous phase exhibits only one magnetic component, which could be identified as $\mathrm{FeB}$ phase and the coercive field is by a factor of 10 higher. This indicates, that the missing high-hyperfine field component in the Mössbauer spectra is attributed to a spin glassy phase containing Fe, which surrounds the by EXAFS identified disordered $\mathrm{Ni}_{3} \mathrm{C}$ phase. As a consequence, the low-field components in carburized, nitrided and oxidized stainless steel films can possibly attributed to stoichiometric FeX phases.

The STPLD process of multi-component inlays (in this case boron-nitride) showed the decrease of the Ability of Multilayer Formation (AMF) by inducing competing diffusion processes. These processes could possibly hinder the formation of grains with spin glassy properties. Hence, future STPLD experiments should be only performed with onecomponent inlays, until the STPLD process is completely understood. 


\subsection{Summary of the pulsed laser deposited films}

In all respects, the pulsed laser deposition of Febased amorphous films exhibit new properties and a huge potential for technical applications. Future experiments should especially be focussed on films prepared by the STPLD technique. Their films show new properties, which can be controlled by the processing parameters: laser beam density, scan velocity, inlay composition and -thickness.

Whereas the magnetron sputtering technique failed, the in this work presented STPLD technique succeeded: by pre-combining an ARMCO target with an graphite inlay, the prediction of Lee et al. [9], that a $\mathrm{NaCl}$-type $\mathrm{FeC}$ phase can be formed, could be confirmed. Films with $20 \mathrm{~nm}$ in thickness were directly deposited on TEM grids and immediately TEM analyzed. A Fe-C bond length of $0.229(3) \mathrm{nm}$ and, thus, a lattice parameter of $a_{0}=0.458(6) \mathrm{nm}$ was obtained. This is in good agreement with Lee's predictions (bond length $0.236 \mathrm{~nm}$ and $a_{0}=$ $0.473 \mathrm{~nm}$ ). Unfortunately, the synthesization of stoichiometric $\mathrm{FeC}$ films failed, which could be originated in inter-diffusion with the a- $\mathrm{SiO}_{2}$ substrate [140] or by a thickness effect [141].

The STPLD of stainless steel - graphite targets lead to the formation of self-organized multilayer films. Together with the solidification disturbance induced by Mo atoms, carbon diffuses through the metal matrix. Local carbon inhomogeneities were induced via the target scan process. This induces local diffusion gradients which leads to an selforganizing effect and which promotes the decomposition of carbon and stainless steel and assists multilayer formation plus the formation of superstructures. By increasing the ion energies by increasing the scan velocity, an additionally implantation of the target materials lead to a locally rearrangement which hinders the overall carbon diffusion in the matrix, but not complete. As a consequence, the size of the superstructure is reduced. The ion implantation could lead to opposing diffusion gradients in the nano-scale regime, which could lead again to a self-organization and the formation of multilayer by decomposing carbon and stainless steel.

The STPLD technique was also used, to synthesize $\mathrm{Al} / \mathrm{C}$ and $\mathrm{Ti} / \mathrm{C}$ multilayer. As a consequence, an exponential correlation between the layer thickness and the atomic number of the metallic component of the target was found.

Unfortunately, the growth mechanism is not fully understood and several experiments have to be carried out to find an empirical formula.

As next step, the inlay material was varied and boron-nitride was used. The boro-nitrided stainless steel film exhibit a verisimilar amorphous phase, which was observed in carburized, nitrided and oxidized stainless steel films. The difference between these amorphous phases is found in magnetism. Whereas the amorphous phase in magnetron and RPLD deposited exhibits two magnetic components and is soft ferromagnetic, the boro-nitrided amorphous phase exhibits only one magnetic component, which could be identified as FeB phase and the coercive field is by a factor of 10 higher. This indicates, that the missing high-hyperfine field component in the Mössbauer spectra is attributed to a spin glassy phase containing Fe. As a result, harder magnetism is observed.

Surprisingly, the synthesization of SS/BN STPLD films only revealed a weak AMF, which could be due to the deposition of BN-droplets. These particulates offer potential wells, on which the adsorbents nucleate. As a consequence, multiayer growth is hindered.

Also RPLD films exhibit the amorphous and soft and ferromagnetic phase. With the aid of this process, the nucleation model was confirmed and further improved.

Laser deposition showed various aspects of carbide formation and exhibit many new properties of iron and stainless steel films. As a consequence, the PLD technique (and especially the STPLD) demonstrates high potential and should be pressed ahead to focus on the development on new materials. 



\section{Chapter 8}

\section{FEL surface processing}

For technical applications, surface processing is sometimes more convenient (concerning layer thicknesses, machining time, time expenditure etc.) than thin film deposition. Furthermore, the STPLD process and the magnetron-sputtering technique exhibit solidification times and cooling rates as crucial factors for film and layer microstructure. For this, FEL surface processing experiments for AISI 310 and AISI 316 were performed at the Jefferson lab. The processing parameters can be found in Table 4.1. As reactive gas nitrogen was used $\left(p_{\text {chamber }}=1 \cdot 10^{5} \mathrm{~Pa}\right)$.

Light microscope images of the AISI 310 and AISI 316 samples after FEL surface processing are depicted in Fig. 8.1.

The melt pool convection determined tracks can

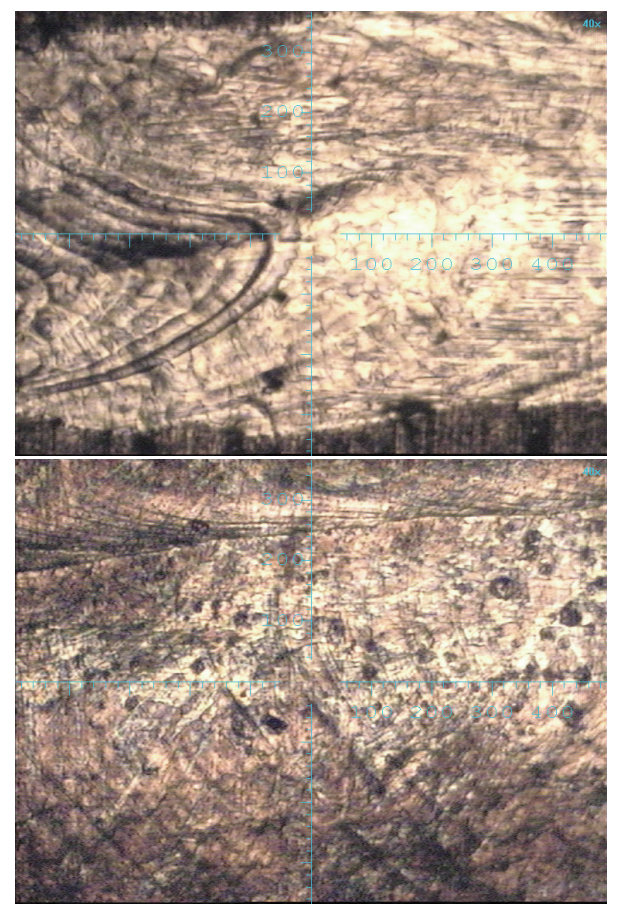

Figure 8.1: Light microscope images of the FEL surface processed stainless steel samples. Top: AISI 310; bottom AISI 316. be clearly seen. Detailed information about the nitrogen transport mechanism and flow behavior in the melt are available in [149].

Fig. 8.2 shows the XRD patterns of the surface processed samples.

Both samples exhibit the $\gamma$ - $(\mathrm{Fe}, \mathrm{Cr}, \mathrm{Ni})$ structure.

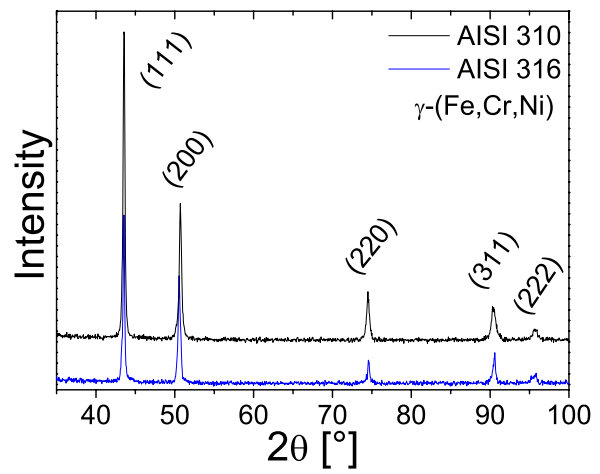

Figure 8.2: GIXRD $\left(2^{\circ}\right)$ spectra of the FEL nitridedfilms. The stainless steel - boron nitride area fractions are given in the graph.

The lattice parameters are derived to $a_{0, A I S I 310}=$ $0.3600(2) \mathrm{nm}$ and $a_{0, A I S I 316}=0.3595(1) \mathrm{nm}$.

By using the equation

$$
a=3.572 \AA+0.7 \AA \times c_{N}^{\gamma}
$$

where $c_{N}^{\gamma}$ is the nitrogen concentration, the nitrogen solved in the $\gamma$-phase can be obtained from the XRD patterns [150]. The $c_{N}^{\gamma}$ value found for the AISI 310 steel was derived to $c_{N}^{\gamma}=4.0 \%$ and $c_{N}^{\gamma}=3.3 \%$ for the AISI 316 sample, respectively.

The CEM spectra are illustrated in Fig. 8.3.

A single line analysis showed in both case three subspectra: two of them are corresponding to the original steel material and one subspectrum corresponds to the formation of a $\mathrm{Fe}_{1-x} \mathrm{~N}_{x}$ phase. The results of the fitting procedures can be obtained in table 8.1.

The nitrogen concentration $c_{N}^{\gamma}$ can also be derived from the doublet subspectra by calculating a binomial distribution with $n=2$ [151]. The obtained $c_{N}^{\gamma}$ 
Table 8.1: Mössbauer results for FEL nitrided stainless steel samples ( $\delta$ - isomer shift, $\Delta$ - the quadrupole splitting for the paramagnetic subspectra, $\epsilon$ the quadrupole splitting for the magnetic subspectra, $B$ hyperfine field, $\Gamma$ - line width (HWHM), $f$ - area fraction).

\begin{tabular}{ccccccr}
\hline sample & subspectrum & $\delta$ & $\Delta, \epsilon$ & $B$ & $\Gamma$ & $f$ \\
& & {$[\mathrm{~mm} / \mathrm{s}]$} & {$[\mathrm{mm} / \mathrm{s}]$} & {$[\mathrm{T}]$} & {$[\mathrm{mm} / \mathrm{s}]$} & {$[\%]$} \\
\hline AISI 310 & $\gamma+\mathrm{N}$ & $-0.05(1)$ & $0.10(5)$ & & 0.13 & $55.0(14)$ \\
& $\gamma+\mathrm{N}$ & & $0.65(27)$ & 0.13 & $5.0(80)$ \\
& $\mathrm{Fe}_{1-x} \mathrm{~N}_{x}$ & & $0.38(7)$ & 0.13 & $40.0(13)$ \\
\hline AISI 316 & $\gamma+\mathrm{N}$ & $-0.05(4)$ & $0.12(2)$ & 0.13 & $58.9(41)$ \\
& $\gamma+\mathrm{N}$ & & $0.68(6)$ & 0.13 & $11.2(33)$ \\
& $\mathrm{Fe}_{1-x} \mathrm{~N}_{x}$ & & $0.35(4)$ & 0.13 & $29.9(40)$ \\
\hline
\end{tabular}

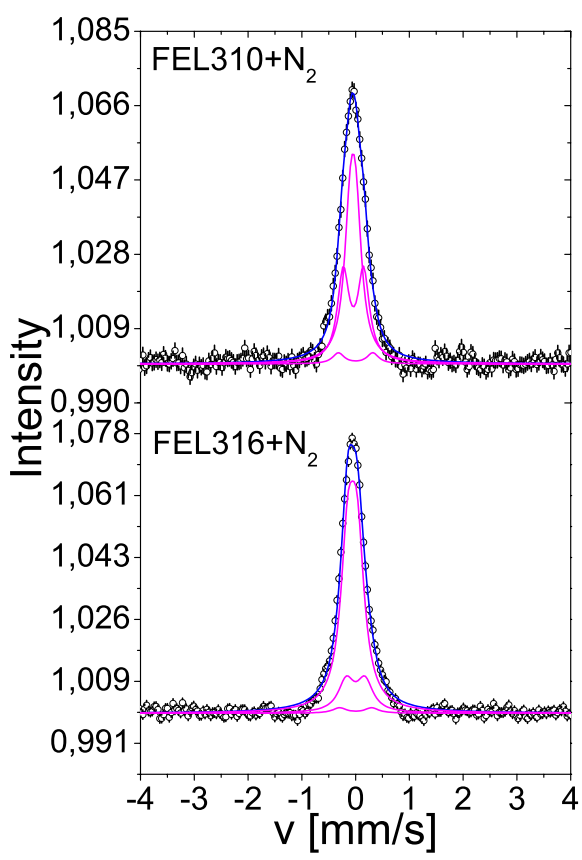

Figure 8.3: Mössbauer spectra of FEL nitrided AISI 310 and AISI 316 samples.

values are $c_{N}^{\gamma}=9.4 \%$ for AISI 310 and $c_{N}^{\gamma}=7.2 \%$ for AISI 316. These values are by a factor of two greater than those derived from the XRD pattern. This might be due to the fact, that the equation 8.1 is only valid for nitrogen in Fe. Thus, 8.1 is only an approximation for nitrided stainless steel films. Unfortunately, no XRD patterns with well defined reflexes were obtained in this work to derive a new correlation.

As a consequence, RBS measurements were carried out, which are shown in Fig. 8.4.

Here, the nitrogen concentration is verisimilar than that derived by Mössbauer Spectroscopy. For AISI 310 a median value of $c_{N}^{\gamma}=9.8 \%$ and $c_{N}^{\gamma}=8.1 \%$ for AISI 316, respectively were found. Furthermore, an oxide surface layer was found in both samples, which should have influence on the hardness of the films.

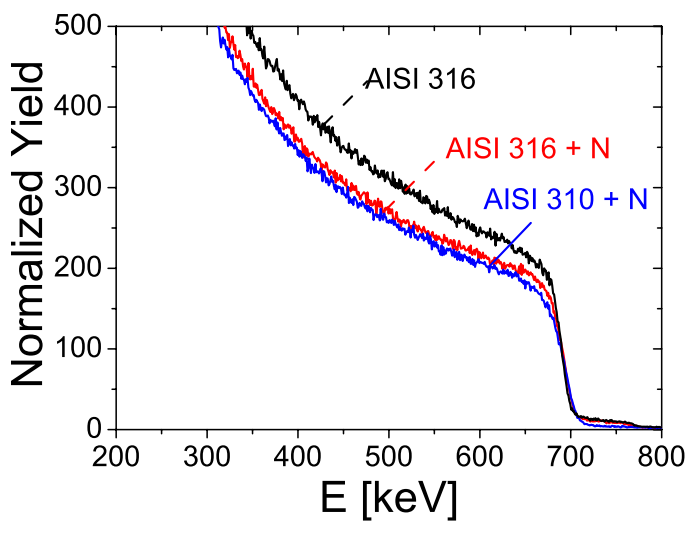

Figure 8.4: RBS spectra of the FEL nitrided AISI 310 and AISI 316 samples.

Microhardness of FEL nitrided stainless steel samples Nanoindentation was performed by using a Fischerscope HV100 [92] with a Vickers diamond in order to investigate the influence of nitriding on the microhardness and the mechanical properties. The maximum indention force was set to $1000 \mathrm{mN}$. Four positions were measured for each sample. The hardness-depth profiles are illustrated in Fig. 8.5.

The hardness distribution close to the surface in-

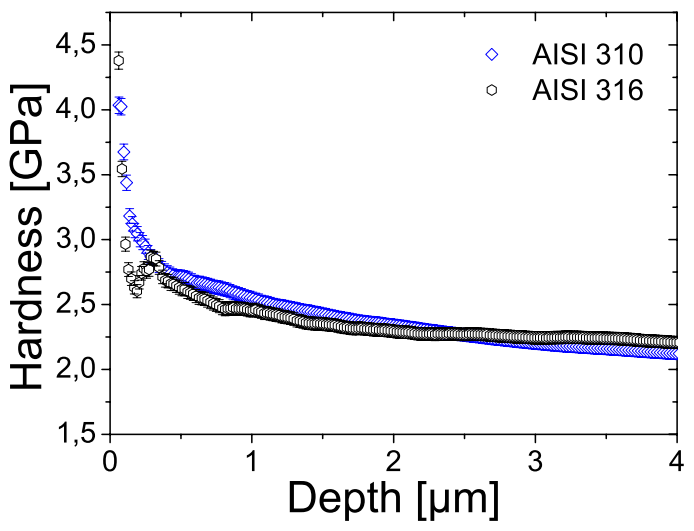

Figure 8.5: Hardness-depth profiles of the FEL nitrided AISI 310 and AISI 316 samples. 
dicates for both steels hardness values, which can be attributed to hard oxide layers, which is in good agreement to RBS.

By using the equation of Jönsson and Hogmark the hardness and the layer thickness were derived [152]:

$$
H_{f}=H_{s}+\left[2 k \frac{t}{d}-\left(\frac{t}{d}\right)^{2}\right]\left(H_{f}-H_{S}\right)
$$

where $t$ is the film thickness, $H_{f}, H_{C}$ and $H_{S}$ the film, the composite and the substrate hardness, $d$ the indentation depth and $k$ an apparatus constant due to the geometry of the Vickers diamond of the indentor (here it was set to 0.14 ).

For AISI 310, a film hardness of 2.64(3) GPa and a layer thickness of $4.85(5) \mu \mathrm{m}$ was found. For AISI 316, the film hardness was derived to $H_{f}=2.49(2) \mathrm{GPa}$; the layer thickness to $5.84(40) \mu \mathrm{m}$.

In comparison to the hardness values of the starting materials, the hardness values of the FEL nitrided stainless steel samples are slightly increased, but are drastically lower than those of Magnetron-sputtered stainless steel films. This could be due to the rapid coagulation of the melt in this process. As a consequence, the nitrogen has not enough time to diffuse through the metal matrix to enhance hardening.

Summarizing, FEL surface processing of stainless steel samples lead to the formation of a well-crystallized surface layers, which have better tribological properties. Unfortunately, not enough nitrogen was incorporated and as a consequence, further experiments have to be done to localize the parameter range (overlap-parameter, laser energy, scan speed, chamber pressure etc.) to obtain amorphous surface layers. 



\section{Chapter 9}

\section{Summary and Outlook}

The synthesization and characterization of amorphous iron and stainless steel films have been the subject of this work. For this, various aspects of the carbide, nitride, oxide and boron-nitride formation and their stability were studied in detail to localize the parameter range of the metastable amorphous state in the given systems.

Intrigued by the predictions of Lee et al. [9], inert sputtering from a pre-combined $\mathrm{Fe}_{50} \mathrm{C}_{50}$ was used to synthesize the stoichiometric NaCl-type FeC phase via Magnetron-sputtering. Unfortunately, geometric effects of the target configuration, hysteresis effects and re-sputtering constitute severe problems to the deposition process. As a consequence of this, the Magnetron sputtering technique seems not to be suitable to synthesize the stoichiometric FeC phase, but the films exhibit carbon contents, which exceed the maximum solubility limits in known carbides. As a result, reactive sputtering of stainless steel - by using methane, nitrogen and oxygen as reactive gas - was performed to synthesize amorphous films or quasi metallic glasses based on conventional steels.

For carburized samples, following results were obtained. All carburized samples showed a broad appearance in the GIXRD patterns revealing the character of amorphous materials. The magnetic properties investigated by means of Mössbauer Spectroscopy and MOKE showed the formation of various phases and carbides at a sputtering temperature of $298 \mathrm{~K}$. In addition a new amorphous and soft ferromagnetic phase was observed. For this phase, a median hyperfine field of $\langle B\rangle=10.3 \mathrm{~T}$ with a width of $\sigma=6.1 \mathrm{~T}$ was obtained from the $P(B)$ distribution of the Mössbauer analysis. The soft ferromagnetic character was derived by MOKE, which revealed a coercive field of approximately 4 Oe. Furthermore, vacuum annealing of this phase showed the carbide reaction $\mathrm{M}_{7} \mathrm{C}_{3} \rightarrow \mathrm{M}_{23} \mathrm{C}_{6} \rightarrow \mathrm{M}_{6} \mathrm{C}$.

To characterize the microstructure of the new amorphous and soft ferromagnetic phase, DSC, FIM, TEM and EXAFS experiments were carried out for carburized samples.

DSC, FIM and TEM showed the quasi metallic glass behavior of these phase, but the microstructure could only be explained by the EXAFS analysis, which revealed a disordered $\mathrm{Ni}_{3} \mathrm{C}$ phase. The magnetism of this phase could be predicted by a LMTO model [119], the order of magnetism could be explained by a core-shell model, describing the nano-particles as consisting of a ferromagnetically core and a disordered surface shell [121,122].

These experiments confirmed a pre-suggested model, which is based on thermodynamical and kinetic remarks of Lu et al. [13] and of Lux and Haubner [113]: the present Fe-based alloy is associated with the deep eutectic point of the Fe-C system. It is well known that compositions around the deep eutectic point are ideal for glass formation in many systems. As a result, glass formation is greatly favored thermodynamically. Further, the minor addition of Mo could promote glass formation in the Fe-C system by suppressing the formation of the primary phase and hindering grain growth. Because of their limited solubility in $\mathrm{Fe}$ carbides, the molybdenum atoms must redistribute and long-range diffusion is required upon solidification. The introduction of a reactive sputtering gas - here the carbon from methane - additionally disturbs the nucleation process by establishing higher probabilities of coordination. This leads to simultaneous rearrangement of different species of atoms, which suppresses the formation of competing ordered phases. As a consequence, phases outside the equilibrium can be formed like the disordered trigonal $\mathrm{Ni}_{3} \mathrm{C}$ phase.

To proof the transferability of this model to the p-block elements $\mathrm{N}$ and $\mathrm{O}$, the reactive Magnetronsputtering technique was used to deposit nitrided and oxidized sample. Indeed, both systems revealed the same amorphous and soft ferromagnetic phase.

As shown in Fig. 6.51, the FeN system can be understood as a perfect Gibbs system in the given parameter range, wherein the $\mathrm{Fe}_{3} \mathrm{~N}$ phase 
represents the minimum of that system. Combined with the shown tendency obtained for the reactive gas flow, any given phase - from amorphous to crystalline - can be reached for Magnetron-nitrided stainless steel films. In fact, amorphous and crystalline phases, such as the new amorphous and soft ferromagnetic and ZnS-type $\gamma^{\prime \prime}-(\mathrm{Fe}, \mathrm{Cr}, \mathrm{Ni}) \mathrm{N}$ phase, were observed.

In contrast, Fig. 6.61 exhibit the $\mathrm{FeO}$ system as a Gibbs plot of a multi-phase system, in which the transition from the amorphous state (domain I) to the crystalline phases (domain II - the formation of $\mathrm{FeO}, \mathrm{Fe}_{2} \mathrm{O}_{3}$ oxides) is depicted. In contrast to the carburized and nitrided amorphous and soft ferromagnetic phase, Mössbauer Spectroscopy and RBS of the oxidized phase indicated the participation of Fe-oxides. As a consequence, the model for the amorphous state for carburized and nitrided samples can not be applied in the same form for oxidized samples.

According to $\mathrm{He}$ et al. [118] and Kodama et al. [135], $\mathrm{NiFe}_{2} \mathrm{O}_{4}$ cores surrounded by spin-glassy surface shells would satisfy the observed results. This hypothesis has to be proven in upcoming EXAFS experiments.

Reconsidering all experiments, it is reasonable to assume, that the model mentioned above is transferable to all p-block elements.

As next step, the verifiability of the model concerning the synthesization mode was investigated. Therefore, the PLD technique was chosen to synthesize high-carbon iron and stainless steel films. For this purpose, the STPLD technique was developed to enlarge the advantages of the PLD. Until the introduction of STPLD, either precasted PLD targets had to chosen or complex rotating target systems were in use. Now, the material and area fraction of the STPLD inlay extend the number of processing parameters.

Whereas the magnetron sputtering technique failed, the in this work presented STPLD technique succeeded: by pre-combining an ARMCO target with an graphite inlay, the prediction of Lee et al. [9], that a NaCl-type $\mathrm{FeC}$ phase can be formed, could be confirmed. Films with $20 \mathrm{~nm}$ in thickness were directly deposited on TEM grids and immediately TEM analyzed. A Fe-C bond length of $0.229(3) \mathrm{nm}$ and, thus, a lattice parameter of $a_{0}=0.458(6) \mathrm{nm}$ was obtained. This is in good agreement with Lee's predictions (bond length $0.236 \mathrm{~nm}$ and $a_{0}=0.473 \mathrm{~nm}$ ). Unfortunately, the synthesization of stoichiometric FeC films failed, which could be originated in inter-diffusion with the $\mathrm{a}-\mathrm{SiO}_{2}$ substrate [140] or by a thickness effect [141].

The STPLD of stainless steel - graphite targets lead to the formation of self-organized multilayer films. Together with the solidification disturbance induced by Mo atoms, carbon diffuses through the metal matrix. Local carbon inhomogeneities were induced via the target scan process. This induces local diffusion gradients which leads to an self-organizing effect and which promotes the decomposition of carbon and stainless steel and assists multilayer formation plus the formation of superstructures. By increasing the ion energies by increasing the scan velocity, an additionally implantation of the target materials lead to a locally rearrangement which hinders the overall carbon diffusion in the matrix, but not complete. As a consequence, the size of the superstructure is reduced. The ion implantation could lead to opposing diffusion gradients in the nano-scale regime, which could lead again to a self-organization and the formation of multilayer by decomposing carbon and stainless steel.

The STPLD technique was also used, to synthesize $\mathrm{Al} / \mathrm{C}$ and $\mathrm{Ti} / \mathrm{C}$ multilayer. As a consequence, an exponential correlation between the layer thickness and the atomic number of the metallic component of the target was found.

Unfortunately, the growth mechanism is not fully understood and several experiments have to be carried out to find an empirical formula, which describes the multilayer thickness.

As next step, the inlay material was varied and boron-nitride was used. The boro-nitrided stainless steel film exhibit a verisimilar amorphous phase, which was observed in carburized, nitrided and oxidized stainless steel films. The difference between these amorphous phases is found in magnetism. Whereas the amorphous phase in magnetron and RPLD deposited exhibits two magnetic components and is soft ferromagnetic, the boro-nitrided amorphous phase exhibits only one magnetic component, which could be identified as FeB phase and the coercive field is by a factor of 10 higher. This indicates, that the missing high-hyperfine field component in the Mössbauer spectra is attributed to a spin glassy phase containing Fe. As a result, harder magnetism is observed.

Surprisingly, the synthesization of SS/BN STPLD films only revealed a weak AMF, which could be due to the deposition of BN-droplets. These particulates offer potentials, on which the adsorbents nucleate. As a consequence, multilayer growth is hindered.

Also RPLD films exhibit the amorphous and soft and ferromagnetic phase. With the aid of this process, the nucleation model was confirmed and further improved. Furthermore, STPLD and RPLD confirmed the independence of the nucleation model on the preparation mode of the films. 
For comparison, the amorphization behavior of surface treated samples was investigated. Therefore, FEL experiments at the Jefferson lab were carried out, wherein the nitrogen incorporation was assisted by laser beam radiation.

FEL surface processing of stainless steel samples lead to the formation of a well-crystallized $\gamma-(\mathrm{Fe}, \mathrm{Cr}, \mathrm{Ni})$ surface layer. FEL treatment of AISI 310 improved the hardness. A film thickness of of $t=4.85(5) \mu \mathrm{m}$ and $H_{f}$ was derived to 2.64(3) GPa. For AISI 316, the film hardness was derived to $H_{f}=2.49(2) \mathrm{GPa}$; the layer thickness to 5.84(40) $\mu \mathrm{m}$. Thus, the FEL revealed its most important advantage: an extremely fast treatment process which allows the formation of functional layers in the micron-scale. However, further experiments have to be done to localize the parameter range (overlap-parameter, laser energy, scan speed, chamber pressure etc.) to obtain amorphous surface layers. 



\section{Bibliography}

[1] T. Masumoto, editor. Material Science of Amorphous Metals. Ohm Pub., Tokyo (1982).

[2] H. Liebermann, editor. Rapidly Solidified Alloys. Marcel Dekker, Inc., New York (1993).

[3] F. Luborsky. Amorphous Metallic Alloys. Butterworths \& Co (Publishers) Ltd. (1983).

[4] A. Inoue. Bulk Amorphous Alloys - Practical Characteristics and Applications. Trans Tech Publications Ltd., Switzerland (1999).

[5] N. Abdel-Salam. Electrochemical Hydrogen Absorption by $\mathrm{Zr}$-Cu-Al-Ni Metallic Glasses. Ph.D. thesis, Universität Dresden (2002).

[6] http://www.hesradio.com/palladium.html.

[7] S. Cusenza et al. Journal of Physics: Condensed Matter, 19(10), 106211 (2007).

[8] H. Binczycka et al. Journal of Physics: Condensed Matter, 18(47), 10561 (2006).

[9] B. J. Lee. Acta Materialia, 54(3), 701 (2006).

[10] B. J. Lee et al. Acta Materialia, 54(17), 4597 (2006).

[11] M. Daw et al. Materials Science Reports, 9(78), 251 (1993).

[12] D. H. Xu et al. Physical Review Letters, 92(24), 245504 (2004).

[13] Z. P. Lu et al. Physical Review Letters, 92(24), 245503 (2004).

[14] V. Ponnambalam et al. Applied Physics Letters, 83(6), 1131 (2003).

[15] H. A. Wriedt et al. Binary Alloy Phase Diagrams. ASM International, Ohio (1996).

[16] H. Massalski et al. Binary Alloy Phase Diagrams. ASM International, Ohio (1996).

[17] M. Ron. Applications of Mössbauer Spectroscopy II. Academic Press, New York (1980).
[18] U. Gonser et al. Amorphous Metals, Glassy Metals II, volume 53, chapter 4, page 93. Springer-Verlag, Berlin, Heidelberg, New York, Tokyo (1983).

[19] F. Habashi. Alloys. Wiley-VCH, Weinheim, New York (1998).

[20] J. K. L. Lai et al. Materials Science and Engineering A-Structural Materials Properties Microstructure and Processing, 379(1-2), 308 (2004).

[21] P. Haasen. Physical Metallurgy. Cambridge University Press, Cambridge (1996).

[22] S. Cusenza et al. Applied Physics A-Materials Science 8 Processing, 93(5), 1 (2008).

[23] D. Turnbull. Contemporary Physics, 10(5), 473 (1969).

[24] D. Turnbull et al. Journal of Chemical Physics, 34(1), 120 (1961).

[25] G. Cargill. Journal of Applied Physics, 41(1), 12 (1970).

[26] J. Bernal et al. Nature, 188(4754), 910 (1960).

[27] C. Janot. Quasicrystals. Clarendon Press, Oxford, 2nd edition (1994).

[28] A. Bracchi. Structural and Magnetic Properties of the Glass-Forming Alloy Nd60Fe30Al10. Ph.D. thesis, Göttingen Graduate School of Physics (2004).

[29] F. Frank. Proceedings of the Royal Society of London Series A-Mathematical and Physical Sciences, 215(1120), 43 (1952).

[30] K. F. Kelton et al. Physical Review Letters, 90(19), 195504 (2003).

[31] A. Inoue et al. IEEE Transactions on Magnetics, 33(5), 3814 (1997).

[32] M. H. Cohen et al. Nature, 189(475), 131 (1961). 
[33] D. Uhlmann. Journal of Non-Crystalline Solids, 7, 337 (1972).

[34] H. Davies et al. Scripta Metallurgica, 8(10), 1179 (1974).

[35] W. A. Johnson et al. Transactions of the Metallurgical Society of AIME, 135, 416 (1939).

[36] M. Avrami. Journal of Chemical Physics, 7, 1103 (1939).

[37] A. Inoue. Materials Transactions JIM, 36(7), 866 (1995)

[38] A. Inoue. Science Reports of the Research Institutes Tohoku University Series A-Physics Chemistry and Metallurgy, 42(1), 1 (1996).

[39] P. J. Desre. Materials Transactions JIM, 38(7), 583 (1997).

[40] F. M. Penning. Proceedings Of The Koninklijke Akademie Van Wetenschappen Te Amsterdam, 33(6/10), 841 (1930).

[41] K. Klabunde. Thin films from free atoms and particles. Orlando, Academic Press, INC. (1985).

[42] J. M. Ngaruiya. Fundamental Processes in Growth of Reactive DC Magnetron Sputtered Thin Films. Ph.D. thesis, Technische Hochschule Aachen (2004).

[43] R. Behrisch et al. Topics in applied physics: Sputtering by particle bombardment III, volume 64. Berlin, Heidelberg, New York, Springer-Verlag (1991).

[44] L. Holland. Vacuum Deposition of Thin Films. John Wiley \& Sons, New York (1956).

[45] D. Bauerle. Laser Processing and Chemistry. Springer, 2nd edition (1996).

[46] K. Mitra et al. Journal of Applied Physics, 80(2), 675 (1996).

[47] http://xfelinfo.desy.de/de/artikel.fel prinzip/2/index.html.

[48] R. L. Mössbauer. Zeitschrift für Physik, 151(2), 124 (1958).

[49] R. L. Mössbauer. Naturwissenschaften, 45(22), 538 (1958).

[50] R. L. Mössbauer. Annual Review of Nuclear Science, 12, 123 (1962).

[51] R. L. Mössbauer. Hyperfine Interactions, 126(1-4), 1 (2000).
[52] H. Frauenfelder. The Mössbauer Effect. W.A. Benjamin, INC. (1962).

[53] H. Wegener. Der Mössbauer-Effekt und seine Anwendungen in Physik und Chemie. Hochschultaschenbücher-Verlag, Bibliographisches Institut, Mannheim (1965).

[54] P. Schaaf. In F. Bassani et al., editors, Encyclopedia of Condensed Matter Physics, pages 20-31. Elsevier, Oxford (2005).

[55] U. Gonser. Mössbauer Spectroscopy, chapter 13. Springer Verlag, Berling-HeidelbergNew York (1986).

[56] G. K. Wertheim. Mössbauer effect: principles and applications. Academic Press, London (1964).

[57] W. Schatz et al. Nuclear Condensed Matter Physics. John Wiley \& Sons, West Sussex (1996).

[58] http://serc.carleton.edu/researcheducation/ geochemsheets/techniques/mossbauer.html.

[59] U. Gonser et al. Hyperfine Interactions, 66(14), 95 (1991).

[60] G. Klingelhöfer et al. Hyperfine Interactions, $69(1-4), 819$ (1991).

[61] P. Schaaf et al. Hyperfine Interactions, 92(14), 1189 (1994).

[62] P. Schaaf et al. Hyperfine Interactions, 57(14), 2095 (1990).

[63] P. Schaaf. Hyperfine Interactions, 111(1-4), 113 (1998).

[64] J. Rehr et al. Rev. Mod. Phys., 72, 621 (2000).

[65] M. Newville. Journal Of Synchrotron Radiation, 8, 322 (2001).

[66] B. Ravel. Journal Of Synchrotron Radiation, 8, 314 (2001)

[67] B. Ravel et al. Journal Of Synchrotron Radiation, 12, 537 (2005).

[68] M. Birkholz. Thin film analysis by X-Ray scattering. Wiley-VCH (2005).

[69] W. H. Bragg. Proceedings of The Royal Society of London Series A-Containing Papers of a Mathematical and Physical Character, 89(610), 246 (1913). 
[70] W. H. Bragg et al. Proceedings of The Royal Society of London Series A-Containing Papers of a Mathematical and Physical Character, 88(605), 428 (1913).

[71] M. Laue. Annalen der Physik, 30(12), 225 (1909).

[72] I. Matt. The principles and practice of Electron Microscopy. University Press, Cambridge, 2nd edition (1997).

[73] D. Williams et al. Transmission Electron $M i$ croscopy. Plenum Press, New York (1996).

[74] J. Leavitt et al. Handbook of Modern Ion Beam Materials Analysis. Material Research Society, Pittsburgh (1995).

[75] M. Uhrmacher et al. Nuclear Instruments 86 Methods In Physics Research Section B-Beam Interactions With Materials And Atoms, 9(2), 234 (1985).

[76] M. Uhrmacher et al. Nuclear Instruments \& Methods In Physics Research Section B-Beam Interactions With Materials And Atoms, 240(1-2), 48 (2005).

[77] T. Osipowicz et al. Nuclear Instruments \& Methods in Physics Research Section B-Beam Interactions with Materials and Atoms, 18(3), 232 (1987).

[78] B. Maurel et al. Nuclear Instruments $\&$ Methods in Physics Research, 197(1), 1 (1982).

[79] I. Vickridge et al. Nuclear Instruments \& Methods In Physics Research Section B-Beam Interactions With Materials And Atoms, 45(1-4), 6 (1990).

[80] K.-P. Lieb et al. Solid State Reactions after Ion Implantation Detected by Nuclear Methods, chapter The Göttingen Ion Implanter IONAS, a versaitle Tool for Nuclear Solid State Studies, page 191. Göttingen (1986).

[81] K.-P. Lieb et al. Plasma Surface Engeneering, chapter depth Profiling of Nitrided Surface Layer by Resonant Nuclear Reaction Analysis, page 1055. DGM Informaionsgesellschaft (1989).

[82] K.-P. Lieb. In J. Stanek, editor, Proceedings of the XXIV Zakopane School of Physics 1990. World Scientific, Singapore (1990).

[83] E. Carpene. Excimer Laser Treatments of Iron, Aluminum and Silicon Substrates in Nitrogen and Methane Atmospheres. Ph.D. thesis, Georg-August Universität Göttingen (2002).
[84] W. H. Bragg et al. Philosophical Magazine, 10(55-60), 318 (1905).

[85] F. Landry et al. Nuclear Instruments $\&$ Methods in Physics Research Section B: Beam Interactions with Materials and Atoms, 179(2), 262 (2001).

[86] A. Kulinska et al. Journal Of Solid State Chemistry, 177(1), 109 (2004).

[87] H. Frauenfelder et al. Perturbed Angular Correlations. North-Holland, Amsterdam (1963).

[88] G. A. Müller et al. Hyperfine Interactions, 151(1), 223 (2003).

[89] G. A. Müller et al. European Physical Journal $B, 48(4), 449$ (2005).

[90] G. A. Müller et al. Hyperfine Interactions, 158(1-4), 137 (2004).

[91] S. Cusenza. Gibbs-Thompson-Effekt in magnetrongesputterten austenitischen Edelstahlschichten. Master's thesis, Georg-August Universität zu Göttingen (2005).

[92] P. Schaaf et al. Surface \& Coatings Technology, 101(1-3), 404 (1998).

[93] S. Cusenza et al. Applied Surface Science, 254(4), 955 (2007).

[94] P. Schaaf et al. Nuclear Instruments $\&$ Methods in Physics Research Section B: Beam Interactions with Materials and Atoms, 53(2), 184 (1991).

[95] http://www.srim.org.

[96] M. Brockmann et al. Journal of Applied Physics, 81(8), 5047 (1997).

[97] J. Ziegler et al. The Stopping and Range of Ions in Solids (2008).

[98] K. Zhang et al. European Physical Journal B, 42(2), 193 (2004).

[99] A. Göhl et al. Journal of Vacuum Science \& Technology B, 18(2), 1031 (2000).

[100] F. Tuinstra et al. Journal of Chemical Physics, 53(3), 1126 (1970).

[101] H. Harima. Journal of Physics: Condensed Matter, 14(38), R967 (2002).

[102] A. Inoue et al. Metallurgical Transactions APhysical Metallurgy And Materials Science, 11(5), 739 (1980). 
[103] P. Schaaf et al. Hyperfine Interactions, 94(14), 2239 (1994).

[104] P. Schaaf et al. Acta Metallurgica et Materialia, 40(2), 373 (1992).

[105] F. S. Li et al. Journal of Physics: Condensed Matter, 7(9), 1921 (1995).

[106] M. Vardavoulias et al. Physica Status Solidi A-Applied Research, 134(1), 183 (1992).

[107] F. Gauzzi et al. Hyperfine Interactions, 69(14), 541 (1991).

[108] P. Schaaf et al. Applied Surface Science, 247(1-4), 607 (2005).

[109] E. Carpene et al. Physical Review B, 65(22), 224111 (2002).

[110] A. C. Ferrari et al. Physical Review B, 61(20), 14095 (2000).

[111] K. Zhang et al. Europhysics Letters, 64(5), 668 (2003)

[112] H. Kaesche. Corrosion of Metals, Physicochemical Principles and Current Problems. Springer-Verlag, Berlin - Heidelberg (2003).

[113] B. Lux et al. Diamond and Diamond-like Films and Coatings, chapter Nucleation and Growth of Low-Pressure Diamond. Plenum Press, New York (1991).

[114] V. Mazurovsky et al. In Third International Conference on Mathematical Modeling and Computer Modeling and Computer Simulation of Materials Technologies MMT (2004).

[115] R. N. Rostovtsev. Metal Science and Heat Treatment, 44, 211 (2002).

[116] A. Heinrich et al. Surface And Interface Analysis, 39(2-3), 240 (2007).

[117] W. T. Elam et al. Physical Review B, 38(1), 26 (1988).

[118] L. He et al. (2007).

[119] L. P. Yue et al. Physical Review B, 62(13), 8969 (2000).

[120] W. T. Pennington. Journal Of Applied Crystallography, 32, 1028 (1999).

[121] E. De Biasi et al. Physical Review B, 65(14), 144416 (2002).

[122] E. De Biasi et al. Physical Review B, 71(10), 104408 (2005).
[123] R. J. Highmore et al. Nature, 339(6223), 363 (1989).

[124] H. J. Lee et al. Journal Of Chemical Physics, $119(18), 9858$ (2003)

[125] D. B. Miracle et al. Philosophical Magazine, 83(20), 2409 (2003).

[126] A. Inoue. Acta Materialia, 48(1), 279 (2000).

[127] M. Rösner-Kuhn et al. Thermochimica Acta, 314(1-2), 123 (1998).

[128] K.-N. Tu et al. Electronic Thin Film Science for Electrical Engineers and Materials Scientists. Macmillan, New York (1992).

[129] X. Zhang et al. Journal of Materials Research, 19(6), 1696 (2004).

[130] Joint Committee for Powder Diffraction Studies (JCPDS) -ICDD - PDF2 Database PCPDFWIN 1.30 (1997).

[131] J. Kesten et al. Hyperfine Interactions, 59(14), 309 (1990).

[132] M. Han et al. Journal of Applied Physics, $89(8), 4619$ (2001).

[133] S. Aggarwal et al. Thin Solid Films, 237(1-2), 175 (1994).

[134] S. Cusenza et al. Journal of Advanced Engineering Materials (in print).

[135] R. H. Kodama et al. Physical Review Letters, $77(2), 394$ (1996).

[136] J. Kunze. Nitrogen and carbon in iron and steel. Akademie Verlag, Berlin (1990).

[137] M. Hillert et al. Metall. Trans. A, 22, 2187 (1991)

[138] P. Schaaf. Progress in Materials Science, 47(1), 1 (2002).

[139] P. Scherrer. Nachr. Ges. Wiss. Göttingen, 2, 98 (1918).

[140] P. Huczkowski. Effect of geometry and composition of $\mathrm{Cr}$ steels on oxide scale properties relevant for interconnector applications in Solid Oxide Fuel Cells (SOFCs). Ph.D. thesis, Technische Hochschule Aachen (2005).

[141] M. Varasteh et al. Journal of Applied Physics, 87(9), 6842 (2000).

[142] M. Schnell. High-resolution Rutherford Backscattering Spectroscopy using an Electronic Analyser. Master's thesis, GeorgAugust Universtät Göttingen (2007). 
[143] C. Borschel. Analysis of thin films and their interfaces using high resolution Rutherford backscattering spectrometry. Master's thesis, Georg-August Universtität Göttingen (2008).

[144] B. F. O. Costa et al. Nuclear Instruments \& Methods in Physics Research Section AAccelerators Spectrometers Detectors and Associated Equipment, 580(1), 404 (2007).

[145] M. S. del Rio et al. Synchrotron Radiation Instrumentation, 705, 784 (2004).

[146] D. L. Windt. Computers In Physics, 12(4), 360 (1998).

[147] N. Millard et al. Surface 85 Coatings Technology, 51(1-3), 446 (1992).

[148] P. Politi et al. Irreversible nucleation in multilayer growth (2002).

[149] D. Höche. Direkte Lasersynthese von Funktionsschichten - Untersuchungen physikalischer Prozesse des Lasernitrierens anhand des Modellsystems TiN. Ph.D. thesis, GeorgAugust Universität Göttingen (2008).

[150] J. Kunze. Nitrogen and Carbon in Iron and Steel - Thermodynamics. Akademie-Verlag (1990).

[151] P. Schaaf et al. Acta Metallurgica et Materialia, 42(9), 3077 (1994).

[152] B. Jönsson et al. Thin Solid Films, 114(3), 257 (1984). 



\section{Danksagung}

Zum Gelingen und zur Durchführung dieser Arbeit haben viele Menschen beigetragen, die mich während der Jahre der Konzentration auf das Thema unterstützt haben und denen ich an dieser offiziellen Stelle danken möchte.

Besonderen Dank gilt meinem Doktorvater Herrn Prof. Dr. Peter Schaaf für die interessante Aufgabenstellung, die weit genug gefasst war, mir nicht die Freiheit bei der Aufgabenstellung der Arbeit zu nehmen und die vielfältige Unterstützung durch immerwährende Diskussionsbereitschaft und Anregungen. Danken möchte ich auch Herrn Prof. Dr. Reiner Kirchheim für die kurzfristige Übernahme des Zweitgutachtens.

Ebenso möchte ich mich auch bei den Institutsmitgliedern des II. Physikalischen Institutes für die stetige Unterstützung und Hilfe, der guten Stimmung und Zusammenarbeit bedanken. Besonders hervorheben möchte ich Daniel Höche für die FEL Bestrahlung meiner Proben und seiner Hilfe bei den Härtemessungen, Christian Lange aka LSL ("Laser Safety Locke") für die Instandhaltung unserer Laser, Hendrik - Henni - Schikora für das Polieren der ADI Proben, sowie Hayo Zutz für das FIB-Praparieren meiner Proben und für die ein oder andere Problemlösung am TEM. Nicht zu vergessen sind in dieser Aufzählung auch Daniel Jürgens und Dr. Michael Uhrmacher für die PAC Untersuchungen. Durch das umsichtige Verhalten aller Institutsmitglieder wurde eine recht familäre Atmosphäre geschaffen, die das Arbeitsklima immer angenehm gestaltete.

Auch den Kollegen anderer Institute möchte ich an dieser Stelle meinen Dank aussprechen; besonders hervorheben möchte ich Priv-Doz. Michael Seibt für die Einführung in die Transmissionsmikroskopie, sowie Prof. Dr. Al-Kassab für die FIB Untersuchungen.

Nicht versäumen will ich, mich bei den Werkstätten des II. Physikalischen Instituts für die große Hilfsbereitschaft bei der Lösung all der vielen Probleme zu bedanken. Stellvertretend seien hier nur die beiden Werkstattleiter Klaus Arndt und Kurt Schemmerling genannt. In diesem Zusammenhang gilt Andreas König besonderen Dank, da meist er mit der Bearbeitung meiner Aufträge beauftragt wurde und er auch das literarische Quartett beim Mensaessen (Daniel - Ocken? -, Christian - sexistische Kackscheiße - Andreas als rote Socke und mir - Salat? Bin ich ne Raupe???) komplettierte.

Die Bewältigung all der kleinen und großen Alltagsprobleme wären ohne Christa Wohlfarth, Ina Watterott, Lucie Hamdi und Heike Ahrens wohl nicht zu meistern gewesen. Vielen Dank für all die Hilfe in den letzten Jahren.

Für aufmerksames Durchsehen meiner Arbeit, anregende Diskussionen bzw. Soforthilfe bei inhaltlichen, chemischen- oder auch Computerfragen möchte ich Andreas Schenk danken. Vor allem jedoch für die kleinen Dinge des Alltags, die uns seit 16 Jahren Freunschaft begleiten und sich auch in beruflicher Produktivität niedergechlagen haben und dies hoffentlich in Zukunft auch tun werden.

Last but not least möchte ich meiner Familie danken. Sie hat mir eine Universitätsausbildung als so selbstverständlich erscheinen lassen und mich von jeher an in 
jedweder Hinsicht unterstützt und an mich geglaubt. Danke für Euren Rückhalt. 


\section{Publikationen}

\section{Bereits veröffentlicht:}

Binczycka, H., Kahle, M., Cusenza, S., Carpene, E., Schaaf, P. - Interstitial ordering of nitrogen and carbon in laser nitrided and laser carburized austenitic stainless steel Journal of Physics-Condensed Matter 18, 47, 2006, 10561

Cusenza, S. et al. - The Gibbs-Thomson effect in magnetron-sputtered austenitic stainless steel films - Journal of Physics-Condensed Matter 19, 10, 2007, 106211

Cusenza, S. et al. - Deposition and properties of high-carbon iron films - Applied Surface Science 254, 4, 2007, 955

Cusenza, S. et al. - Amorphous stainless steel coatings prepared by reactive magnetronsputtering from austenitic stainless steel targets - Journal of Applied Physics A, http://dx.doi.org/10.1007/s00339-008-4685-x

Cusenza, S. et al. - Nitrided amorphous stainless steel coatings deposited by reactive magnetron sputtering from an austenitic stainless steel target - Advanced Engineering Materials, 2008, in print

\section{Eingereicht bzw. in Planung}

Schaaf, P., Cusenza, S., Bamberger, M., Amran, Y., Weiss, K., Meier, K., Wasmuth, U., Hofman, M. - Phase Transition Kinetics in Austempered Ductile Iron - Proceedings of the MSE08, 2008

Fingerle, A., Herminghaus, V., Cusenza, S., Schaaf, P. - Determining the Granular Velocity Distribution based on the Mössbauer Effect - Physical Review B

Cusenza, S. et al. - EXAFS study of an amorphous and soft ferromagnetic phase in thin AISI 316 stainless steel films - Physical Review B

Cusenza, S. et al. - Magnetron-oxidized amorphous stainless steel films, Acta Materialia, 2008

Cusenza, S. et al. - Control of magnetic properties and self organized structures in carbon - stainless steel multilayer films - Physical Review B

Cusenza, S. et al. - Formation and Characterization of NaCl-Type FeC Films - Physical Review Letters

Binczycka, H., Kahle, M., Cusenza, S., Carpene, E., Schaaf, P. - The formation of an amorphous ferromagnetic phase by laser carburized austenitic stainless steel - Physical Review B 



\section{Curriculum vitae}

Name:

Geburtstag:

Adresse:

Staatsangehörigkeit:

Familienstand:

\section{Ausbildung}

1986-1990

1990-1992

1992-1999

1999-2005

2006-2008
Salvatore Cusenza

18. Dezember 1979 in Wolfsburg/Niedersachsen

Krumme Stücke 14, 38446 Wolfsburg

italienisch

ledig

Eichendorff-Schule I (Grundschule)

Eichendorff-Schule II (Orientierungsstufe)

Ratsgmynasium Wolfsburg

Diplom-Studium der Physik an der Georg-August Universität zu Göttingen

Promotionsstudium an der Georg-August Universität zu Göttingen

\section{Berufliche Tätigkeiten}

2002-2004

2002-2005

2006-2008
Werksstudent der Volkswagen AG Wolfsburg (PM-5/PM-V Verfahrenstechnik, F\&E)

Nebentätigkeiten während des Studiums: Studentische Hilfskraft im physikalischen Anfängerpraktikum

Wissenschaftlicher Mitarbeiter am II. Physikalischen Institut der Georg-August Universität zu Göttingen 